\title{
Enhancement Strategies in NMR
}

\section{Spectroscopy}

\author{
Dissertation \\ for the award of the degree \\ "Doctor rerum naturalium" \\ of the Georg-August-Universität Göttingen \\ within the doctoral program "chemistry" \\ of the Georg-August University School of Science (GAUSS) \\ submitted by \\ Eibe Behrend Dücker
}

from Twistringen

Göttingen 2018 


\section{Thesis Committee}

Prof. Dr. Christian Griesinger

NMR-based Structural Biology, Max Planck Institute for Biophysical Chemistry, Göttingen

Prof. Dr. Franc Meyer

Institute of Inorganic Chemistry, University of Göttingen

\section{Members of the Examination Board}

Reviewer:

Prof. Dr. Christian Griesinger

NMR-based Structural Biology, Max Planck Institute for Biophysical Chemistry, Göttingen

Second Reviewer:

Prof. Dr. Franc Meyer

Institute of Inorganic Chemistry, University of Göttingen

\section{Further Members of the Examination Board}

Prof. Dr. Marina Bennati

Institute of Organic and Biomolecular Chemistry, University of Göttingen

Dr. Stefan Glöggler

Max Planck Institute for Biophysical Chemistry, Göttingen

Dr. Tim Schäfer

Institute of Physical Chemistry, University of Göttingen

Dr. Florian Ehlers

Institute of Physical Chemistry, University of Göttingen

Date of the oral examination: $7^{\text {th }}$ of May 2018 


\section{Affidavit}

I hereby declare that I wrote this thesis Enhancement Strategies in NMR Spectroscopy on my own and without the use of any other then the cited sources and tools.

Göttingen, $11^{\text {th }}$ of April 2018

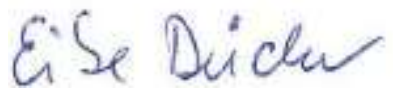

Place, Date

Signature 


\section{Summary}

Hyperpolarization methods have become a powerful assistance technique to NMR spectroscopy in order to improve its sensitivity and consequently reduce the required measurement time to achieve a satisfactory signal-to-noise ratio. In the SABRE (Signal Amplification by Reversible Exchange) polarization technique, an advancement of parahydrogen-induced polarization (PHIP), hyperpolarization is transferred from parahydrogen molecules to heterocyclic small molecules via a metal template without inducing a chemical change to the substrate. Therein, the efficiency of this polarization transfer depends on several factors, such as substrate exchange rate and, most importantly, the magnitude of the magnetic field, at which the hyperpolarization experiment is performed.

In the course of this work, the SABRE technique was applied to a variety of $\mathrm{N}$-heterocyclic compounds in order to assess the effect of changes in the substitution pattern and the basicity of the substrate molecule to the attainable proton enhancement. The hyperpolarization experiments were further performed using three different polarization transfer catalysts to validate if the effects caused by these changes are uniform for diverse catalyst systems.

It was experimentally determined that the magnetic fields, at which the polarization transfer is most efficient is primarily determined by the employed polarization transfer catalyst and is comparable for a wide variety of substrates. Differences in the substitution pattern of the $\mathrm{N}$-heterocyclic substrates cause a change in the relative enhancement levels of individual substrate protons. This is due to the mechanism of polarization transfer, in which the substrate protons adjacent to the coordination site experience direct transfer of magnetization from the parahydrogen nuclei, while the remaining substrate protons obtain magnetization by an intramolecular redistribution process depending on the scalar couplings within the molecule. A change in the substitution pattern changes this system of coupling, which therefore affects the dynamics of the redistribution process. Furthermore, the effect of a substituent-induced change in basicity on the attainable enhancement was examined. While a strong influence of the employed substituent was observable, the effect was not uniform for the examined catalyst systems and a clear dependence of the enhancement on the basicity of the substrate molecule could not be derived.

Lastly, the synthesis of a solid-phase bound $N$-heterocyclic carbene ligand to be employed in a SABRE catalyst system for heterogeneous transfer of polarization was approached. Due to synthetical difficulties, the target molecule had to be repeatedly reassessed. In the final stage of this work, however, preliminary hyperpolarization experiments with polymer-bound iridium complexes were successfully conducted. 


\section{Contents}

Summary

Contents

vii

1 Introduction 1

1.1 Hyperpolarization methods 1

1.2 Signal Amplification By Reversible Exchange 3

1.2.1 The Fundamentals of SABRE 5

$\begin{array}{lll}\text { 1.2.2 Advancements and Applications in SABRE } & 7\end{array}$

1.2.2.1 SABRE Ligand Systems 8

$\begin{array}{lll}\text { 1.2.2.2 Substrate Examination Studies } & 13\end{array}$

1.2.2.3 Polarization Transfer to Heteronuclei 17

1.2.2.4 SABRE Polarization Transfer at High Field 20

$\begin{array}{lll}\text { 1.2.2.5 } & \text { SABRE Detection at Low Field } & 21\end{array}$

$\begin{array}{lll}\text { 1.2.2.6 Heterogeneous SABRE Catalysts } & 24\end{array}$

1.2.2.7 Theoretical Descriptions and Computational Analyses 25

1.3 Employed Catalyst Systems 26

1.3.1 Crabtree's Catalyst 26

1.3.2 N-Heterocyclic Carbenes $\quad 27$

1.4 Objective of this Work 29

2 Theory 30

$\begin{array}{lll}2.1 & \text { Parahydrogen } & 30\end{array}$

2.2 Chemical and Phenomenological Description of SABRE 31

2.3 Kinetic Description of SABRE Processes 32

2.4 Strong Coupling and Level Anti-Crossings $\quad 35$

2.4.1 Strong Coupling in NMR 36

2.4.2 Nuclear Level Anti-Crossings in SABRE model complexes 37

2.4.2.1 $A A^{\prime} M$ three-spin system $\quad 39$

$\begin{array}{lll}\text { 2.4.2.2 } & A A^{\prime} M M^{\prime} \text { four-spin system } & 39\end{array}$

2.4.2.3 $\quad \mathrm{AA}^{\prime} \mathrm{MM}^{\prime} \mathrm{P}$ five-spin system $\quad 41$

2.5 Mechanism of the Polarization Transfer 42

3 Results and Discussion $\quad 44$

3.1 SABRE Hyperpolarization Experiments $\quad 44$

3.1.1 Experimental Setup $1 \quad 44$

3.1.1.1 Pyrazole and its Derivatives $\quad 44$

3.1.1.2 Pyridine Derivatives $\quad 50$

3.1.1.3 Discussion of Experimental Setup 1 52

3.1.2 Experimental Setup $2 \quad 55$

3.1.2.1 Substrates examined using the $\operatorname{Ir}(C O D)\left(P y-d_{5}\right)\left(P C y-d_{33}\right)$ precatalyst 56

3.1.2.2 Substrates examined as a comparison of $\mathrm{PCy}_{3^{-}}$, IMes- and SIMes-containing $\begin{array}{ll}\text { catalysts } & 62\end{array}$

$\begin{array}{ll}\text { 3.1.2.3 Discussion of catalyst performances } & 72\end{array}$

3.1.2.4 Discussion of Experimental Setup $2 \quad 73$

$\begin{array}{lll}3.2 & \text { Chemical Synthesis } & 79\end{array}$

$\begin{array}{lll}\text { 3.2.1 Synthesis of a solid-phase bound SABRE catalyst } & 79\end{array}$

3.2.1.1 Definition of initial target molecule 80 
3.2.1.2 Synthesis of imidazolium chloride 1

3.2.1.3 Change of synthesis target 82

3.2.1.4 Modified polymer beads $\quad 83$

$\begin{array}{lll}\text { 3.2.1.5 Outlook } & 84\end{array}$

4 Materials and Methods $\quad 87$

4.1 Hyperpolarization Experiments $\quad 87$

$\begin{array}{lll}4.1 .1 & \text { Materials } & 87\end{array}$

4.1.2 Preparation of Parahydrogen $\quad 87$

4.1.3 Setup 1: The Shaking Table 87

$\begin{array}{lll}\text { 4.1.3.1 Experimental Setup } & 87\end{array}$

4.1.3.2 Sample Preparation $\quad 89$

4.1.3.3 Experimental Procedure $\quad 89$

$\begin{array}{lll}\text { 4.1.3.4 Evaluation } & 90\end{array}$

4.1.4 Setup 2: The Bruker Para-Hydrogen Polariser $\quad 91$

$\begin{array}{lll}\text { 4.1.4.1 Experimental Setup } & 91\end{array}$

4.1.4.2 Determination of Transfer Parameters 93

4.1.4.3 Determination of Polarization Field 94

4.1.4.4 Sample Preparation 96

4.1.4.5 Experimental Procedure 96

$\begin{array}{lll}\text { 4.1.4.6 Evaluation } & 97\end{array}$

4.1.5 NMR Data of Examined Substrates $\quad 97$

$\begin{array}{lll}\text { 4.1.5.1 } & \text { Pyrazole and its related compounds } & 97\end{array}$

$\begin{array}{ll}\text { 4.1.5.2 } & \text { Pyridine and its related compounds } \\ \end{array}$

$\begin{array}{llr}4.2 & 105\end{array}$

$\begin{array}{lll}4.2 .1 & 105\end{array}$

$\begin{array}{ll}\text { 4.2.2 Chromatography } & 105\end{array}$

$\begin{array}{ll}\text { 4.2.3 NMR Spectroscopy } & 105\end{array}$

$\begin{array}{ll}\text { 4.2.4 Mass Spectrometry } & 105\end{array}$

4.2.5 Experimental Details 106

4.2.5.1 [(1-2-n:5-6-n)-cycloocta-1,5-diene]di(pyridine- $\left.d_{5}\right)$-iridium(I) tetrafluoroborate 106

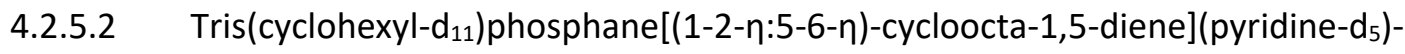
iridium(I) tetrafluoroborate $\quad 107$

$\begin{array}{lll}\text { 4.2.5.3 N,N-dibenzyl-(4-bromo-2,6-dimethylanilin) } & 108\end{array}$

4.2.5.4 4-(N,N-dibenzylamino)-3,5-dimethylphenylboronic acid 109

4.2.5.5 Methyl 4-(4'-(N,N-dibenzylamino)-3',5'-dimethylphenylmethyl)benzoate 110

4.2.5.6 Methyl 4-(4'-amino-3',5'-dimethylphenylmethyl)benzoate 111

4.2.5.7 N-(2,4,6-trimethylphenyl)-N'-(4-(4-(methylcarboxy)phenylmethyl)-2,6dimethylphenyl)formamidine 112

4.2.5.8 1-(2,4,6-trimethylphenyl)-3-(4-(4-(methylcarboxy)phenylmethyl)-2,6dimethylphenyl)-4-hydroxy-4,5-dihydroimidazolium chloride 113

4.2.5.9 [4-(Bromomethyl)phenoxymethyl]polystyrene 114

4.2.5.10 [4-(((2,4,6-trimethylphenyl)imidazoline)methyl)phenoxymethyl]-polystyrene bromide $\quad 115$

5 Bibliography 116

$\begin{array}{llr}6 & \text { List of Figures } & 124\end{array}$ 
$7 \quad$ List of Schemes

8 List of Abbreviations 131

9 Pulse Programs 133

9.1 zg.ebdu_trigin 133

9.2 ph_zg_noload_noempty 134

Curriculum Vitae

136 





\section{Introduction}

Nuclear magnetic resonance (NMR) spectroscopy poses a powerful and versatile tool that is being widely employed in various branches of the natural sciences. In chemistry labs all over the world, NMR spectrometers are used on a daily basis in order to characterize the products of synthetic reactions and to gain insight into stereochemistry and relative conformations of small molecules. In the field of structural biology, high field NMR magnets aid in the structure determination of large biological polymers, for which the vast number of resonances is made distinguishably by spreading over two-, three- and even higherdimensional high-resolution NMR spectra. Using advanced computational methods, this approach can be extended to exceed rigid DNA, RNA and protein structures and to give access to motion studies of individual domains inside these massive macromolecules. Furthermore, magnetic resonance imaging (MRI), which relies on the same principles as NMR, is nowadays a readily available method of examination in the medical sciences and can be used in order to obtain high resolution, three-dimensional scans of the human brain and body. Since patients are not exposed to ionizing radiation, MRI is considered a very mild, yet effective, non-invasive probing technique.

The inherent sensitivity issue of NMR spectroscopy owed to the small population difference of nuclear states at thermal equilibrium has, however, since the beginning limited the examination of low concentration samples, originating from either limited solubility, limited availability or limited abundance of the target nuclei. A great deal of work is being invested up to this date in order to overcome this impediment using various different techniques and approaches. Superior superconductors are being developed to create stronger magnetic fields for employment in NMR spectrometers, yet the acquirement and maintenance of such instruments is costly and more often than not new premises have to be constructed in order to house these devices not only because of their mere size but also their magnetic stray field. To increase the sensitivity of existing equipment, time-tested methods are incorporated on a regular basis. For more insensitive heteronuclei, e.g. ${ }^{13} \mathrm{C}$ and ${ }^{15} \mathrm{~N}$, these include the application of specific pulse sequences like INEPT (Insensitive Nuclei Enhanced by Polarization Transfer) [1, 2], which facilitate transfer of polarization from the more readily excited hydrogen nuclei or the employment of isotope labelling to increase their inherently low natural abundance [3].

\subsection{Hyperpolarization methods}

Further efforts have gone into the creation of so-called non-Boltzmann population distributions, also referred to as hyperpolarization, in which the population differences between the nuclear spin states by far exceed those at thermal equilibrium. Hyperpolarization techniques include Spin-Exchange Optical Pumping (SEOP) of noble gases [4], Dynamic Nuclear Polarization (DNP) using transfer of electron polarization [5, 6] and Parahydrogen-Induced Polarization (PHIP) [7]. For SEOP, electronic states of the alkali metals rubidium or cesium in the gas state are selectively excited using circularly polarized 
photons from a laser source. In the following step, this electronic polarization is converted into polarization of ${ }^{129} \mathrm{Xe}$ or ${ }^{3} \mathrm{He}$ nuclei via a collision pathway. In an alternative approach termed Metastability Exchange Optical Pumping (MEOP), ${ }^{3} \mathrm{He}$ gas is polarized without the need for alkali metals, by initial excitation of the atomic gas into a metastable electronic state using an RF pulse, thereby enabling a subsequent direct interaction with the laser photons [8]. Hyperpolarized noble gases created using these methods can subsequently, among other uses, be employed in the examination of the respiratory tract by MRI, in the investigation of material surfaces or in the transfer of polarization to other nuclei, for example via the spin Overhauser effect. Insight into these methods, as well as further applications and the theoretical background were summarized by Goodson [9].

Electronic excitation is also the source of hyperpolarization in the DNP technique. In Overhauser-DNP, the Larmor frequency of a radical electron is selectively irradiated and the corresponding electronic transition thereby saturated. Hyperpolarization of the targeted nuclei is then achieved via a cross-relaxation mechanism of electron and nucleus. In order to maximize the signal enhancement, the polarization step in DNP is often performed at reduced magnetic fields, where the relaxation-based transfer is more efficient. The magnitude of the preserved magnetization is further strongly dependent on the nuclear relaxation rates, which are significantly increased due to the presence of the employed radical molecules, necessitating a rapid sample transfer from the intermediate to the high magnetic field of the NMR magnet. Accordingly, special equipment has been developed to efficiently facilitate the rapid sample relocation, coining the term shuttle-DNP $[10,11]$. As relaxation rates of heteronuclei are commonly much lower than for protons, isotopically labelled molecules that were hyperpolarized using DNP can be employed to study the substrate distribution and metabolization in time-intensive in vivo MRI experiments [12]. A plethora of further applications have been reported on, reviews of which are given, among others, by Günther [13] and Maly et al. [14].

For parahydrogen-induced polarization, the nuclear singlet spin state of hydrogen molecules, in which the two nuclear spins are aligned in an antiparallel fashion, is exploited in order to generate high levels of hyperpolarization. Such $\mathrm{H}_{2}$ molecules, which are referred to as parahydrogen $\left(p \mathrm{H}_{2}\right)$, exhibit a total nuclear spin of $I=0$, which renders them unsusceptible to radio frequency manipulation and thus NMR silent unless the chemical equivalency of the two hydrogen atoms is lifted. In classic PHIP experiments, a pairwise hydrogen transfer reaction to an unsaturated molecule is performed using para-enriched hydrogen gas. If the two nuclei remain coupled and the symmetry between them is broken due to a transfer to non-equivalent positions inside a molecule or a coordination complex, their polarization evolves and can subsequently be examined using NMR. In the recorded spectra, the resonances of the former $\mathrm{pH}_{2}$ nuclei exhibit strongly increased intensities owed to their non-Boltzmann magnetization. The appearance of these and selected other signals in the spectra is dependent on the experimental procedure, as two different protocols have been established, namely ALTADENA (Adiabatic Longitudinal Transfer After Dissociation 
Engenders Nuclear Alignment) and PASADENA (Parahydrogen And Synthesis Allow Dramatically Enhanced Nuclear Alignment), in which the hydrogenation reaction is performed at low-field outside the spectrometer or directly inside the NMR magnet, respectively [15]. Determined by the selected procedure, different nuclear spin terms contribute to the observable magnetization and depending on the prevalent magnetic field, a transfer of polarization to other nuclei in the substrate is possible. A thorough description of these effects and of the underlying theoretical aspects is given by Natterer and Bargon [15]. PHIP is routinely applied to the studies of metal complexes and catalytic reactions, as the immense signal enhancement allows for the observation of trace amounts of intermediate products or coordination compounds. It is further used in mechanistical studies to verify the pairwise transfer of hydrogen nuclei, which is, in most [16] cases, a requirement for the occurrence of PHIP. In a series of reviews, the group of Duckett has described in detail the fields of application for this polarization technique [17-20].

A

B
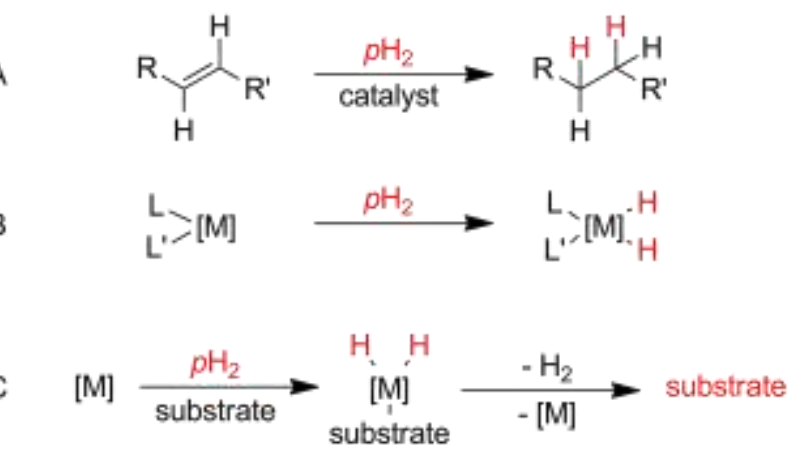

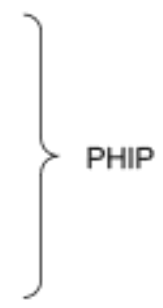

SABRE

Scheme 1: Differences between PHIP and SABRE. In reactions A and B, the parahydrogen nuclei are transferred into coupled, yet unequal positions in the product compounds indicated by the distinguishable substituents $R$ and $R^{\prime}$ as well as the ligands $L$ and $L^{\prime}$. Consequentially, the polarization on the nuclei becomes observable and enhanced resonances of the highlighted proton spins can be recorded by NMR spectroscopy. In reaction $\mathrm{C}$, the substrate and the $\mathrm{pH}_{2}$ nuclei are only temporarily associated via a metal template, resulting in a transfer of polarization to the spins of the unmodified substrate, observable by NMR. [M] indicates a non-specified metal coordination complex.

\subsection{Signal Amplification By Reversible Exchange}

In PHIP spectra, the molecule exhibiting the signal enhancement is not the substrate that was placed in the sample tube or reaction container, but the product compound formed in the hydrogenation reaction. Consequently, in order to study a specific target molecule using the PHIP effect, it must first be created by the addition of a $\mathrm{pH}_{2}$ molecule to an unsaturated precursor compound, which is often not readily available. Furthermore, the substrate molecules are used up in the course of the hydrogenation reaction, thereby limiting the number of experiments that can be conducted using a single prepared sample before the employed compound has been completely hydrogenated. Discoveries reported on by the group of Duckett at the University of York in 2009 overcame both of these limitations. In their initial studies, they found that certain iridium complexes exhibit the ability to reversible bind hydride ligands stemming from parahydrogen molecules as well as pyridine and similar compounds that were employed as substrates. The recorded NMR spectra showed increased signal intensities for the resonances of the unaltered substrate, indicating a 
hyperpolarization pathway from $\mathrm{pH}_{2}$ to the molecule of interest without the occurrence of a chemical modification. As the substrate is consequentially not used up, replenishing the $\mathrm{pH}_{2}$ in the sample solution allows for the conduct of additional experiments using the same sample. This effect was termed Signal Amplification By Reversible Exchange (SABRE). A simplified schematic of the PHIP and SABRE reactions is given in Scheme 1.

In their first publication on the topic of SABRE, Atkinson et al. observed the reversible binding effect for the iridium complexes $\left[\operatorname{Ir}\left(\mathrm{PR}_{3}\right)_{2}(\mathrm{py})_{2}(\mathrm{H})_{2}\right] \mathrm{BF}_{4}$ and $\left[\operatorname{Ir}\left(\mathrm{PR}_{3}\right)(\mathrm{py})_{3}(\mathrm{H})_{2}\right] \mathrm{BF}_{4}$ (where $\mathrm{py}=$ pyridine, $\mathrm{R}=$ phenyl, $p$-tolyl or $p$-methoxyphenyl), which were prepared from the precursor complexes $\left[\operatorname{Ir}(\mathrm{COD})\left(\mathrm{PR}_{3}\right)_{2}\right] \mathrm{BF}_{4}$ (where $\mathrm{COD}=$ cyclooctadiene) by addition of pyridine and hydrogen gas [21]. Upon application of $\mathrm{pH}_{2}$ to the solution at high-field, the hydride resonances of the complexes exhibited PHIP enhancement and a transfer of this polarization to ${ }^{15} \mathrm{~N}$ nuclei in isotopically labelled pyridine was achieved using an INEPT based protocol. The nitrogen resonance of free, unmodified pyridine molecules subsequently experienced a signal enhancement by a factor of 120 .

In a report published shortly after, Adams et al. describe signal enhancement obtained for ${ }^{1} \mathrm{H},{ }^{13} \mathrm{C}$ and ${ }^{15} \mathrm{~N}$ nuclei in free pyridine and nicotinamide molecules [22] using the iridium complex $\left[\operatorname{Ir}(\mathrm{H})_{2}\left(\mathrm{PCy}_{3}\right)(\text { substrate })_{3}\right] \mathrm{BF}_{4}$ (where $\mathrm{Cy}=$ cyclohexyl and substrate = pyridine, nicotinamide), which was obtained from $\left[\operatorname{Ir}(\mathrm{COD})\left(\mathrm{PC}_{3}\right)(\mathrm{MeCN})\right] \mathrm{BF}_{4}$ upon treatment with $\mathrm{pH}_{2}$ and an excess of the substrate. The corresponding experiments were performed under ALTADENA conditions, meaning that the polarization step, in which the $\mathrm{pH}_{2}$ is dissolved in the solution, is executed at reduced magnetic fields outside of the spectrometer and the sample subsequently inserted into the NMR magnet to acquire the desired spectrum. Resonances within a single substrate showed varying levels of polarization and both absorptive and emissive signals were detected. Further hyperpolarized substrates included 3-fluoropyridine, nicotine, pyridazine, quinoline, quinazoline, quinoxaline and dibenzothiophene and hyperpolarization of ${ }^{19} \mathrm{~F}$ and ${ }^{31} \mathrm{P}$ nuclei was also reported. Moreover, applicability to MRI was demonstrated, significantly increasing the signal-to-noise ratio (SNR) while reducing the required acquisition time.

The third publication in short succession reported on the $\mathrm{pH}_{2}$-derived hyperpolarization achieved using $\left[\operatorname{Ir}\left(\mathrm{H}_{2}\left(\mathrm{PCy}_{3}\right)(\mathrm{py})_{3}\right] \mathrm{BF}_{4}\right.$, which was derived from the $\mathrm{BF}_{4}$-paired version of Crabtree's catalyst $[23,24],\left[\operatorname{Ir}(\mathrm{COD})\left(\mathrm{PC}_{3}\right)(\mathrm{py})\right] \mathrm{BF}_{4}$. It was found that a polarization step at low magnetic field was necessary in order to facilitate the spontaneous polarization transfer, i.e., without nuclear spin manipulation using radio frequency (RF) pulses, from the $\mathrm{pH}_{2}$-derived hydride ligands to the ${ }^{1} \mathrm{H},{ }^{13} \mathrm{C}$ and ${ }^{15} \mathrm{~N}$ nuclei in the analyte. Exposure of the sample to parahydrogen at high-fields inside the NMR magnet yielded no enhancement of the corresponding resonances [25]. Interestingly, it was also found that the relative sign and the level of hyperpolarization of the individual resonances depended on the exact magnitude of the polarization field, as spectra of samples polarized at earth's field ( $0.5 \mathrm{Gs})$ exhibited a different signal pattern compared to those polarized within the stray field of the NMR magnet ( 5 to $100 \mathrm{Gs}$ ). This effect was observed for ${ }^{1} \mathrm{H}$ as well as ${ }^{13} \mathrm{C}$ nuclei. The authors 
furthermore examined the catalyst loading dependence on the obtained enhancement by varying the catalyst-to-substrate ratio from 1:5 to $1: 1000$ and found that within these limits, higher percentage catalyst loading resulted in higher achieved enhancement. Additionally, the achievable signal enhancements for pyridine were compared when several further iridium complexes containing different versions of the phosphine ligand were employed as polarization transfer catalysts. Highest levels of enhancements were found for dicyclohexylphenylphosphine, followed by tricyclohexylphosphine and triisopropylamine.

Shortly after, Adams et al. gave a theoretical description of the phenomenon, based on a 4-spin model complex consisting of the two former parahydrogen nuclei and two substrate spins [26]. Therein, the transfer of polarization is attributed to the presence of a scalar coupling between the $\mathrm{pH}_{2}$ nuclei and those of the substrate molecules. It was further postulated that the efficiency of the transfer depends on the size of these couplings and the chemical shifts. From this it was concluded that the effect is time- and field dependent, meaning that the level of achieved polarization depends on the lifetime of the active polarization transfer catalyst as well as on the magnetic field strength, at which the polarization transfer takes place. An exhaustive analysis of field dependences for various spin terms generated in the polarization transfer step was presented, yet no correlation to experimental data was produced.

\subsubsection{The Fundamentals of SABRE}

For SABRE experiments, the sample is prepared by dissolving a suitable iridium-based precatalyst and an excess of the substrate of interest in an appropriate solvent. Routinely, methanol- $d_{4}$ is employed, although other solvent systems have also been tested [27-33]. The sample is then transferred into a suitable container, where it is exposed to parahydrogenenriched hydrogen gas to generate the active polarization transfer catalyst. An exemplary schematic of this reaction for the three precatalysts utilized in this work is depicted in Scheme $2[21,22,25,34]$.

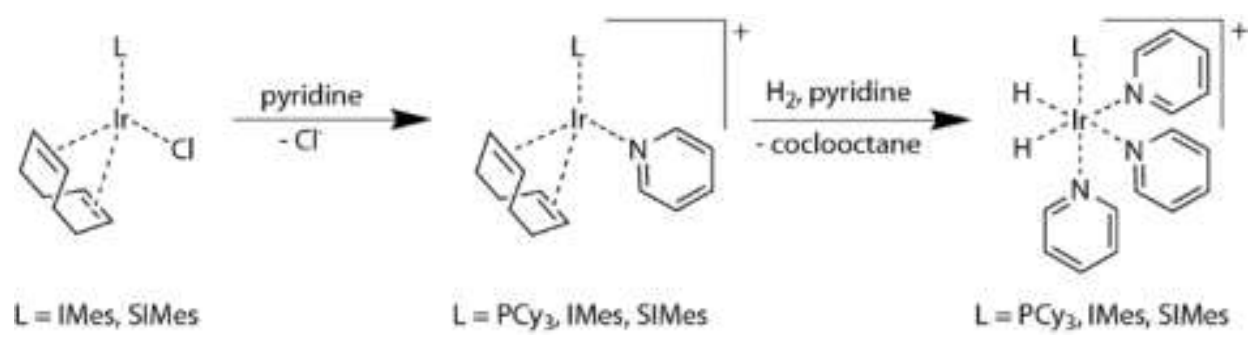

Scheme 2: Preparation of the active polarization transfer catalyst starting from the precatalysts $\left[\operatorname{Ir}(\mathrm{COD})\left(\mathrm{PC} \mathrm{P}_{3}\right)(\mathrm{py})\right]^{+}$and $[\operatorname{Ir}(\mathrm{COD})(\mathrm{NHC}) \mathrm{Cl}](\mathrm{NHC}=\mathrm{IMes}, \mathrm{SIMes})$. Here, pyridine is used as an exemplary substrate compound.

SABRE hyperpolarization experiments are usually performed in one of the two following ways:

For the so-called shaking method $[21,22,25,35]$, the sample solution is placed and activated inside a pressure-resistant NMR tube, to which then an overpressure of parahydrogen is 
applied. In order to dissolve the hydrogen gas in the sample solution, the sample tube is vigorously shaken for several seconds at a low-field position within the stray field of the NMR magnet, for which the strength of the prevalent magnetic field is known. Subsequently, the sample tube is quickly inserted into the NMR magnet and the desired NMR experiments are performed.

For the bubbling method, the sample solution is placed inside a reaction container, into which a flow of $\mathrm{pH}_{2}$ gas can be passed via an immersion tube and for which the prevalent magnetic field strength in the low-field range can be adjusted either by variation of the distance to the NMR magnet or by field modulation using an electromagnetic coil. When the desired magnetic field is set, parahydrogen is bubbled through the solution in order to dissolve the gas molecules in the sample solution. After the bubbling procedure, the sample is mechanically $[36,37]$ or pneumatically [29] transferred to the center of the NMR magnet in order to perform the NMR experiments.

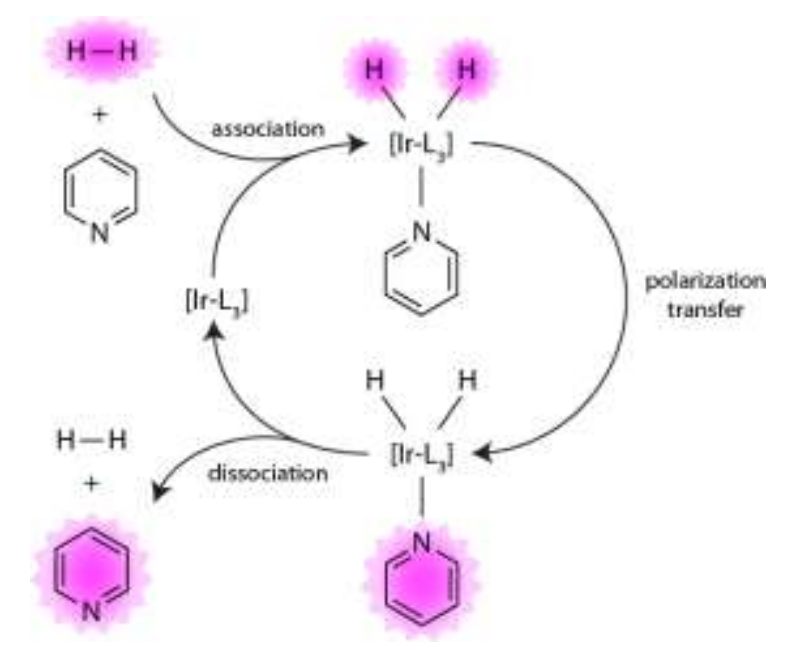

Scheme 3: Simplified schematic of the catalytic cycle of the SABRE polarization transfer. The highlighted molecules are in a non-equilibrium nuclear spin state and are therefore considered hyperpolarized. Pyridine is depicted as an exemplary substrate.

The experimental procedures that serve to dissolve the $\mathrm{pH}_{2}$ in the sample solution are referred to as the polarization step, as here the analyte and the parahydrogen nuclei are brought into contact with each other by temporary association via the active polarization transfer complex. During the lifetime of the complex, the nuclear polarization of the $\mathrm{pH}_{2}$ spins is transferred to the substrate nuclei (Scheme 3) via the extended J-coupling network. After dissociation, the substrate molecule remains in the hyperpolarized state and can be examined in NMR experiments. Depending on the employed catalyst and the magnetic field strength at which the polarization step was performed (commonly referred to as the polarization field), the substrate resonances exhibit strongly enhanced absorptive or emissive resonances. Owed to these two parameters, the corresponding signal intensities and patterns are highly variable, both relative to each other and in absolute terms. For unmodified SABRE protocols, the polarization field usually exhibits a strength of below 250 Gs. 
In contrast to classic PHIP experiments, the $\mathrm{pH}_{2}$ nuclei are not incorporated into the substrate molecules as the hydrogenation reaction is energetically unfavorable due to the aromatic nature of the commonly employed analytes. Therefore, the substrate remains chemically unchanged in the polarization process and can be repolarized numerous times. Furthermore, the $\mathrm{pH}_{2}$-derived polarization is not made accessible by breaking the symmetry between the former parahydrogen nuclei but is spontaneously transferred to the substrate spins at low-field via the scalar coupling network of the active polarization transfer catalyst without additional spin manipulation using the NMR radio frequency coils. Lastly, the hyperpolarization is generated mainly in the form of longitudinal magnetization, which is detectable using standard one-scan $90^{\circ}$ hard-pulse experiments and which can therefore by employed in a variety of unmodified NMR pulse sequences. An extensive theoretical description of SABRE is presented in Section 2.

\subsubsection{Advancements and Applications in SABRE}

Since the initial publications, a great deal of work has been invested in the application, improvement and analysis of the SABRE technique with contributions from numerous different research groups [38]:

Dücker et al. set out to experimentally investigate the polarization field dependence of the SABRE enhancement [35]. To that end, the authors conducted polarization transfer experiments for pyridine and a number of pyrazole derivatives at various magnetic field strengths in the stray field of the NMR magnet, using Crabtree's catalyst $\left[\operatorname{Ir}(\mathrm{COD})\left(\mathrm{PC}_{3}\right)(\mathrm{py})\right] \mathrm{BF}_{4}$ as the precatalyst. It was found that chemical differences in the substrates had only very limited influence on the profile of the determined polarization field dependences and that the results obtained for each pyrazole derivative exhibited maximum enhancement at fields of 30-40 and $140 \mathrm{Gs}$, between which a transition from absorptive to emissive signals was observed. The results for pyridine showed a similar appearance, yet the curves were displaced on the $y$-axis for individual protons, so that ortho and para protons primarily gained polarization with a negative phase, while the meta resonances almost exclusively exhibited positive intensities. These experimentally determined field dependences were contradictory to those previously predicted by Adams et al. [26]. It was further reported that a dilution of the analyte pyridine by its deuterated analog resulted in increased levels of enhancement observed for the analyte resonances. Additionally, polarization transfer to the solvent hydroxy resonance was reported, presumably caused by $\mathrm{H} / \mathrm{D}$ exchange between the solvent and substrates exhibiting an acidic $\mathrm{NH}$ proton.

In a computational study, Pravdivtsev et al. identified the presence of Level Anti-Crossings (LAC) at low magnetic fields of $<250 \mathrm{Gs}$ as the key factor of the polarization field dependence in SABRE [37]. In the case of Crabtree's catalyst, the employed phosphine ligand exhibits a significant scalar coupling between its phosphorus atom and the hydride ligands, creating additional LACs which are the cause for the observed bimodal field dependence curves [35]. Numerical calculations for an eight-spin system consisting of the hydride ligands in the initial singlet state, the ${ }^{31} \mathrm{P}$ spin of the phosphine ligand and the five proton spins of pyridine as the 
analyte produced an excellent agreement with experimentally obtained field dependences. For catalyst systems that exhibit no ${ }^{31} \mathrm{P}-{ }^{1} \mathrm{H}$ coupling, only a single field range of highest polarization transfer efficiency is reported.

In order to be able to conduct SABRE experiments simply and reproducibly, Mewis et al. reported on the development of the automatic Parahydrogen Polariser unit, designed and manufactured by Bruker BioSpin UK [29] (Figure 1). The Polariser is connected to a flow injection probe and can transfer samples from a low-field to the high-field position using a pneumatic flow system. At the low-field position, the device is equipped with a mixing chamber, in which $\mathrm{pH}_{2}$ can be applied to the sample solution. Surrounding the mixing chamber is a copper solenoid, using which the magnetic field strength at the low-field position can be modulated. The functionality of the equipment was demonstrated by examining the effects of the polarization field, the $\mathrm{pH}_{2}$ pressure, the $\mathrm{pH}_{2}$ exposure time and the catalyst concentration on the level of achieved enhancement for the analyte nicotinamide. Furthermore, the effect of the catalyst concentration on the effective analyte $\mathrm{T}_{1}$ time constants was examined, showing increased relaxation rates at higher complex concentrations. Additionally, higher order spin terms created in the process of hyperpolarization and their behavior under variant experimental parameters were analyzed with the OPSY (Only Para-hydrogen SpectroscopY) sequence [39], using which individual longitudinal higher order terms can be selectively observed. Polarization of nicotinamide in ethanol- $d_{6}$ and a $1: 1$ ethanol- $d_{6} / D_{2} \mathrm{O}$ mixture was also performed with reduced efficiency owed to divergent reaction kinetics for the ligand exchange in these solvents. Direct hyperpolarization of ${ }^{13} \mathrm{C}$ nuclei in nicotinamide was found to be most efficient at $0 \mathrm{Gs}$.

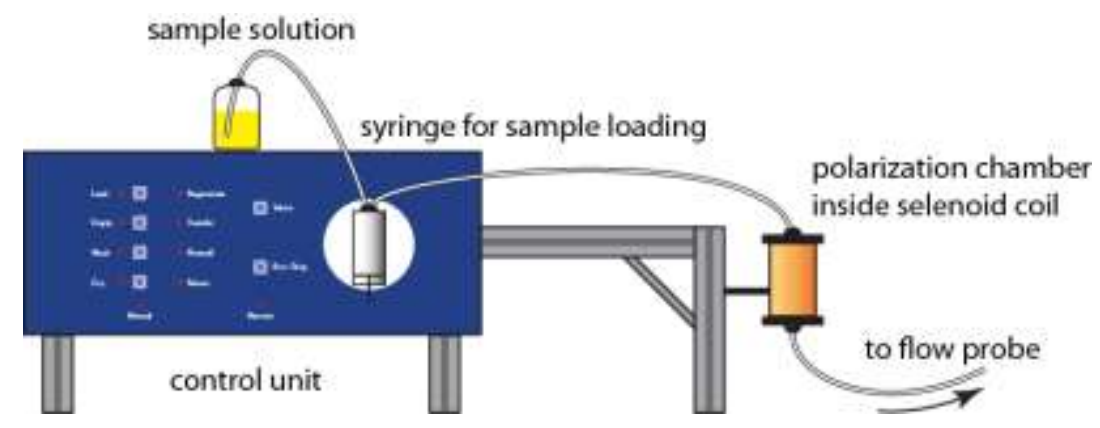

Figure 1: Setup of the Bruker Parahydrogen Polariser. Using the syringe, the sample solution can be transferred into the polarization chamber, where the polarization field can be adjusted by the surrounding solenoid. To achieve dissolution of $\mathrm{pH}_{2}$, a flow of the gas can be applied to the mixing chamber and subsequently the solution can be transferred into an attached flow probe for NMR examination.

\subsubsection{SABRE Ligand Systems}

The SABRE activity of the polarization transfer catalysts depends strongly on the types of ligands in the coordination sphere of the complex. Especially the non-exchanging, axially bound, sterically demanding phosphine and $N$-heterocyclic carbene ligands play an essential role and several types of these ligands have been studied:

As the first NHC-carrying precatalyst, the iridium complex $[\operatorname{Ir}(\mathrm{COD})(\mathrm{IMes}) \mathrm{Cl}]$ (IMes = 1,3bis(2,4,6-trimethylphenyl)imidazole-2-ylidene (1, Figure 2)) was examined by Cowley et al. 
and was reported to exhibit superior catalytic polarization transfer activity compared to the previously employed phosphine complexes [34]. Upon its treatment with pyridine and $\mathrm{H}_{2}$, the dihydride complex $\left[\operatorname{Ir}(\mathrm{H})_{2}(\mathrm{IMes})(\mathrm{py})_{3}\right]$ is formed, which was assessed to be the active catalyst species. In this complex, two pyridine ligands and the two hydrides are located in the equatorial plane and were found to exchange with free pyridine and $\mathrm{H}_{2}$ in solution. The third pyridine ligand is bound in the axial position opposite of the IMes ligand and does not undergo exchange. An increase in temperature decreased the level of attained enhancement for this complex, while for Crabtree's catalyst higher enhancements were achieved at elevated temperatures. This suggests an optimal window of ligand exchange rates for efficient transfer of polarization. Also, higher $\mathrm{pH}_{2}$ pressures yielded higher levels of enhancement and the same is true for higher percentage catalyst loading. Reducing the size of the proton spin system by employing 3,4,5-trideuteropyridine as the analyte resulted in higher enhancement factors for the remaining protons, presumably as the finite amount of polarization was shared among fewer nuclei. A polarization field dependence evaluation was performed using the new catalyst and pyridine as a substrate, giving $60 \mathrm{Gs}$ as the most effective field strength for the hyperpolarization of pyridine para protons.

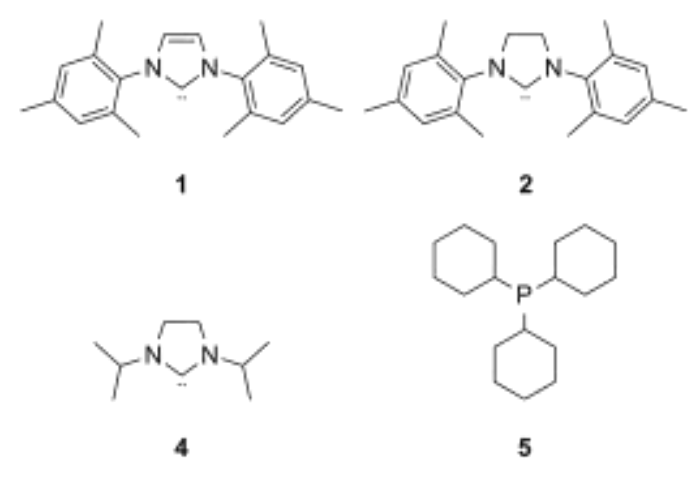

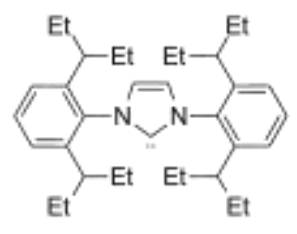

3

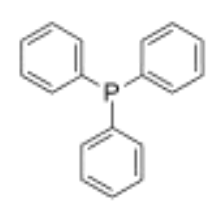

6

Figure 2: Assorted ligands utilized in SABRE related studies including IMes (1), SIMes (2), PCу (5) and PPh (6).

Van Weerdenburg et al. analyzed eleven NHC ligands in order to determine their effect on SABRE when employed in $\left[\operatorname{Ir}(\mathrm{H})_{2}(\mathrm{NHC}) \text { (substrate) }\right)_{3}$ ]-type polarization transfer catalysts [40]. To that end, they compared an analogue of the Tolman Electronic Parameter (TEP) [41] and the buried volume (\% $\left.\mathrm{V}_{\text {bur }}\right)$ [42] exhibited by the different $\mathrm{NHCs}$, which describe the electronic donor ability and the steric demand of a ligand, respectively. It was found that even though the ligands show a strongly deviating polarization transfer potential among them, the range of exhibited TEP values, which describe the change in the carbonyl stretching frequency of a CO ligand imposed by coordination of a ligand of interest, is extremely narrow $\left(<3 \mathrm{~cm}^{-1}\right)$ and significantly smaller than for phosphine-type ligands $(\sim 20$ $\mathrm{cm}^{-1}$ ). It was therefore concluded that the electronic effects have only a secondary influence on the attained enhancements. The ligand exchange rate ( $\left.k_{\text {off }}\right)$ for pyridine showed, however, a correlation to the buried volume of the NHC ligand. NHCs containing a saturated backbone, e.g. SIMes (2), exhibited a higher level of flexibility, which increases their steric bulk and results in higher exchange rates. Experimental results suggest that optimal rates of 
ligand exchange are close to the value of $10.4 \mathrm{~s}^{-1}$ that was found for the unsaturated IMes ligand, which achieved highest levels of signal enhancement and for which an increase as well as a decrease in the exchange rates resulted in lower levels of observed polarization. Additionally, the chemical shifts exhibited by complex bound analytes do not correlate with the electronic parameters of the NHC but are rather caused by the presence or absence of aromaticity in the substituents of the NHC ligands, which was postulated to have a large influence on the polarization transfer efficiency. These findings were later extended by producing a satisfactory correlation between the observed substrate exchange rate and the $\pi$-accepting ability parameter (PAAP) [43] of the NHC ligands, which describes their electronic parameters detached from their $\sigma$-donating ability [44]. Deviations were observed for NHCs exhibiting exceptionally large steric bulk (3) or a strictly aliphatic constitution (4). A more thorough discussion will be presented in chapter 2.5 .

Similar studies on NHC ligands were performed by Lloyd et al., who also found that larger NHC ligand size correlates with a weaker binding of substrates and vice versa, which modulates the ligand exchange rate [45]. They further found that changes in the ligand ${ }^{15} \mathrm{~N}$ chemical shift due to metal coordination do not correlate with the binding affinity between complex and ligand. Low temperature examination of ligand exchange pathways suggests the exchange of the hydride ligands taking place via the temporary 16-electron complex $\left.\left[\operatorname{Ir}(\mathrm{H})_{2}(\mathrm{NHC}) \text { (substrate }\right)_{2}\right]^{+}$resulting from the dissociation of one of the substrate ligands. The vacant coordination site is then occupied by $\mathrm{H}_{2}$, giving the intermediate $\left[\operatorname{Ir}(\mathrm{H})_{2}\left(\mathrm{H}_{2}\right)(\mathrm{NHC})(\text { substrate })_{2}\right]^{+}$complex. $\mathrm{H}_{2}$ exchange is therefore limited by the dissociation of the substrate. Subsequently, increased rates of hydride exchange resulted in an increase of observed enhancement. The intermediate 16-electron complex is further able to coordinate solvent molecules, resulting in the formation of an unsymmetrical complex that exhibits PHIP enhanced hydride resonances. For all examined NHC ligands, a change in temperature caused a change in the observable polarization levels, as the substrate exchange rate depends on the energy of the system. Each NHC catalyst exhibits highest signal enhancements at a different temperature, where the ligand exchange rate is in the optimal range depending on the ligand properties. The substrate $T_{1}$ time constants are furthermore effectively reduced by coordination to the polarization transfer catalyst, necessitating a compromise between longer coordination times for highest efficiency of polarization transfer and shorter coordination times in order to reduce the level of relaxation. The optimal rates of pyridine loss stated here are between 0.5 and $0.08 \mathrm{~s}^{-1}$ per mole of complex $\left(0.25 \mathrm{~s}^{-1}\right.$ for the most efficient IMes ligand), corresponding to optimal catalyst lifetimes of around $0.25 \mathrm{~s}$. Furthermore, complexes of different NHCs exhibited deviating field dependences when employed as polarization transfer catalysts.

Fekete et al. examined the iridium complexes $\left[\operatorname{Ir}(\mathrm{H})_{2}(\mathrm{IMes})\left(\mathrm{PPh}_{3}\right)(\mathrm{NCMe})_{2}\right]^{+}$and $\left[\operatorname{Ir}(\mathrm{H})_{2}(\mathrm{IMes})\left(\mathrm{PC}_{3}\right)(\mathrm{NCMe})_{2}\right]^{+}$for their applicability as SABRE polarization transfer catalysts, which simultaneously incorporate the two ligand types utilized in well-studied SABRE catalysts, i.e., phosphines and $\mathrm{N}$-heterocyclic carbenes [27]. In solutions containing equal 
amounts of acetonitrile and pyridine, the thermodynamically favored mixed complex $\left[\operatorname{Ir}(\mathrm{H})_{2}(\mathrm{IMes})\left(\mathrm{PR}_{3}\right)(\mathrm{NCMe})(\mathrm{py})\right]^{+}(\mathrm{R}=\mathrm{Cy} 5, \mathrm{Ph} 6)$ was formed instead of the $(\mathrm{NCMe})_{2}$ or $(\mathrm{py})_{2}$ analogs. For the $\mathrm{PPh}_{3}$ complex, ligand exchange was found to be initiated by dissociation of acetonitrile, while for the $\mathrm{PC}_{3}$ species, pyridine exchange was dominant. When employed as SABRE catalysts, both complexes efficiently facilitated transfer of polarization from $\mathrm{pH}_{2}$ to pyridine and exhibited enhancement maxima at comparable magnetic field strengths. The enhancement levels obtained for the $\mathrm{PC}_{3}$ versions were comparable to those determined for the thoroughly investigated $\left[\operatorname{Ir}(\mathrm{H})_{2}(\text { IMes)(substrate })_{3}\right]^{+}$catalyst. Furthermore, the complex-bound ligands as well as acetonitrile molecules gained signal intensity from $\mathrm{pH}_{2}$. Employment of pyridine- $d_{5}$ as the analyte changed the observed enhancements on the ligands, increasing the signal intensities for acetonitrile and the IMes ligand while decreasing the polarization detected on the phosphine protons. Polarization transfer to the latter was therefore presumed to not be from the hydride ligands directly, but from previously enhanced pyridine ligands. Using a $45^{\circ}$ excitation pulse, strong PHIP derived signals with antiphase character were observed on the hydride ligands of the mixed complex, while no hydride resonances originating from symmetrical species were detected. Employing a mixture of pyridine- $d_{5}$ and pyridine- $h_{5}$ in the absence of acetonitrile also induced asymmetry in the formed complexes, which consequently also exhibited PHIP enhanced hydride resonances. It was not mentioned if a signal enhancement via the SABRE pathway was observed on the hydride resonances.

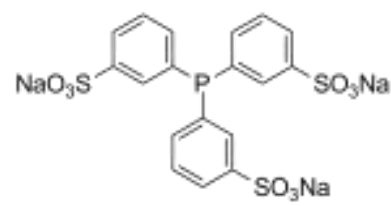

$7 \mathbf{a}$

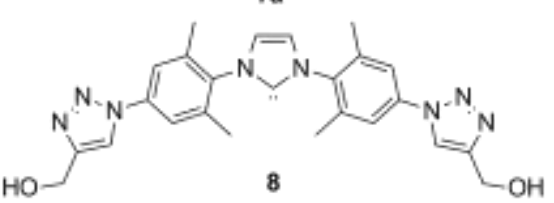

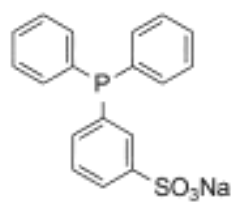

$7 \mathrm{~b}$

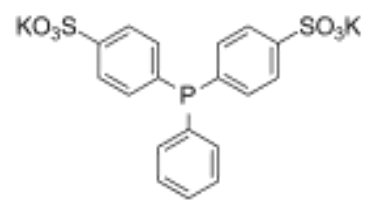

$7 \mathrm{c}$

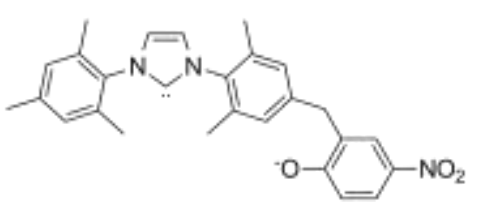

10

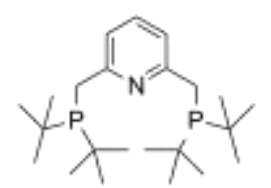

11

Figure 3: Assorted modified phosphine and NHC ligands. Ligands 7a-c, $\mathbf{8}$ and $\mathbf{9 a} \mathbf{a} \mathbf{b}$ were synthesized to create polarization transfer catalysts with a higher polarity to be used in water-based solvent systems, while ligand $\mathbf{1 0}$ served the purpose of creating a neutral catalytic complex for application of SABRE in non-polar solvents.

To improve the SABRE results in aqueous solution, the same authors strived to synthesize a more water soluble polarization transfer catalyst [32]. The first series of tested complexes consisted of three catalysts of the type $\left[\operatorname{Ir}(\mathrm{H})_{2}(\mathrm{NCMe})(\mathrm{py})(\mathrm{IMes})(\mathrm{L})\right]$, where $L$ represents mono-, di- and trisulfonated variants of the $\mathrm{PPh}_{3}$ ligand (7a-c, Figure 3). In methanol- $d_{4}$, the catalysts exhibiting the sulfonated phosphine ligand achieved lower levels of enhancement compared to the complex of the unmodified $\mathrm{PPh}_{3}$, with highest enhancements obtained by the monosulfonated version with a factor of $\sim 400$. Compared to MeOD as a solvent, Catalyst activity was found to be 5 times lower in ethanol- $d_{6}$, while in water and a water-ethanol mixture, the activity was poor, exhibiting no SABRE enhancement. Furthermore, high levels 
of $\mathrm{H} / \mathrm{D}$ exchange were observed in $\mathrm{D}_{2} \mathrm{O}$ and ethanol- $d_{6}$ for the hydride ligands and pyridine. Consequently, a second series of catalysts was synthesized which employed modified IMes (8) and SIMes $(9 a, b)$ ligands, into which peripheral triazole rings were incorporated in order to increase the polarity of the resulting complex. Again, good SABRE activity was observed in methanol- $d_{4}$ and - to a lower extent - in ethanol. When a mixture of $67 \% D_{2} \mathrm{O}, 3 \% \mathrm{DMSO}$ and $30 \%$ ethanol was employed as solvent, the polarization transfer decreased or stopped altogether. This effect was attributed to the presence of DMSO, which was added to improve hydrogen solubility, yet which might also coordinate irreversibly to the complexes. Employing $\mathrm{D}_{2} \mathrm{O}$ or $3: 7$ ethanol/ $\mathrm{D}_{2} \mathrm{O}$ as the solvent resulted in strongly reduced enhancements, rendering direct hyperpolarization in these solvents impractical.

An experimental modification for the application of the SABRE protocol to aqueous solutions was described by Zeng et al. [30], in which 3-amino-1,2,4-triazin and [ $\operatorname{Ir}(\mathrm{COD})(\mathrm{IMes}) \mathrm{Cl}]$ were dissolved in methanol and the active catalyst species was subsequently generated by application of $\mathrm{pH}_{2}$. Following this, water was added, and the methanol fraction reduced to $10 \%$ by heating to $40{ }^{\circ} \mathrm{C}$. The sample was then exposed to $\mathrm{pH}_{2}$ at a field of $65 \mathrm{Gs}$ at a temperature of $54.4{ }^{\circ} \mathrm{C}$, yielding a signal enhancement of 170 in a mostly aqueous solution. Application of this protocol to the analytes pyridine, isoniazid and pyrazinamide proved, however, unsuccessful.

In order to extend the applicability of SABRE to non-polar solvents, Ruddlesden et al. synthesized an IMes derived NHC ligand, in which one of the 2,4,6-trimethylphenyl substituents was replaced by a 2-methylene-4-nitrophenolate moiety (10) and using which a neutral polarization transfer catalyst was generated [33]. Activation of the complex to form the operative catalyst species using an excess of analyte and $\mathrm{H}_{2}$ proved to take significantly longer in the aprotic solvents benzene- $d_{6}$, tetrahydrofuran- $d_{8}$ and dichloromethane- $d_{2}$ as well as protic ethanol- $d_{6}$, as satisfactory levels of polarization transfer were detected after $48 \mathrm{~h}$, yet a 3 to 5 -fold improvement on these values was observed after 24 days. Over this time period, no signs of degrading or H/D exchange were detectable. In methanol, however, deuteration takes place over this elongated time span, resulting in significantly reduced enhancement factors observed after 24 days compared to after $48 \mathrm{~h}$. Benzene proved to be an excellent solvent for the conduct of non-polar SABRE experiments.

Holmes et al. tested an iridium PNP pincer complex employing the 2,6-bis(di-tertbutylphosphinomethyl)-pyridine ligand (11) for its applicability to the SABRE protocol [46]. While the active polarization transfer catalyst $\left[\left(\mathrm{C}_{5} \mathrm{H}_{3} \mathrm{~N}\left(\mathrm{CH}_{2} \mathrm{P}\left({ }^{t} \mathrm{Bu}\right)_{2}\right)_{2}\right) \operatorname{Ir}(\mathrm{H})_{2}(\mathrm{py})\right]^{+}$was formed after treatment with pyridine and $\mathrm{H}_{2}$, this complex coordinates only one analyte molecule at a time in contrast to other SABRE catalysts, which usually bind two to three substrate molecules. In combination with the exhibited low ligand exchange rates, this resulted in comparatively low levels of hyperpolarization for the employed analyte pyridine as against previously examined polarization transfer catalysts. An increase in temperature resulted in higher ligand exchange rates, which lead to slightly higher enhancement levels attained after polarization at $65 \mathrm{Gs}$, yet surprisingly also to lower levels of enhancement when the 
polarization step was performed at $0.5 \mathrm{Gs}$. Furthermore, deuteration of the pyridyl ring and the methylene groups resulted in an increase in signal intensity for the examined analytes.

\subsubsection{Substrate Examination Studies}

The requirement for substrate molecules to be susceptible to the SABRE technique is the presence of a donor atom in their molecular structure, with which the compound can reversibly bind to the catalytic complex. Despite the structural similarity of the analytes imposed by this necessity, the achieved levels of hyperpolarization vary greatly among the examined substrates. Several studies therefore placed their focus on the employed analytes themselves, often with the intent of maximizing the attained enhancement levels for the selected compound of interest:

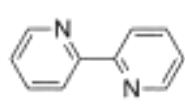

2,2 -bipyridine

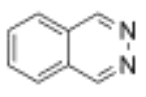

phthalazine

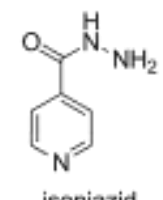

isoniazid

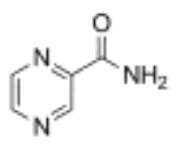

pyrazinamide

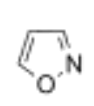

isoxazole 1-methyl-1,2,3-triazole

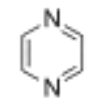

pyrazine
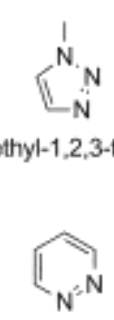

pyridazine
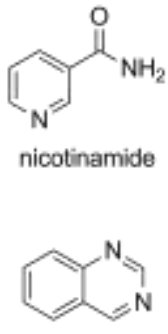

quinazoline
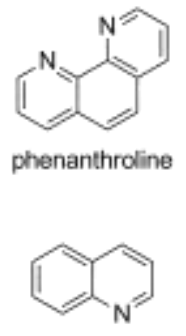

quinoline

Figure 4: Molecular structures of chemical compounds referred to in this section.

Quinoline (Figure 4) was examined using the $[\mathrm{Ir}(\mathrm{COD})(\mathrm{IMes}) \mathrm{Cl}]$ precatalyst in a study by Lloyd et al., probing the applicability of SABRE to the acquisition of two-dimensional spectra [47]. All proton resonances in the analyte exhibited SABRE and enhanced 1D NOE transfer was achieved between two protons attached to the annular ring systems. Using the ${ }^{1} \mathrm{H}$ OPSY-COSY sequence [39], a ${ }^{1} \mathrm{H}-{ }^{1} \mathrm{H}$ correlation spectrum of a $2 \mu \mathrm{mol}$ quinoline sample was obtained within 16 minutes. Using ultrafast COSY [48], a two-dimensional spectrum of $6 \mu \mathrm{mol}$ analyst was recorded using only one hyperpolarization step, allowing assignment of the entire substrate proton network. Using a $60 \mu \mathrm{mol}$ sample, ${ }^{13} \mathrm{C}$ spectra were recorded in single scans, either by direct ${ }^{13} \mathrm{C}$ observation after hyperpolarization at fields close to $0 \mathrm{Gs}$ in a magnetic shielding chamber in order to minimize the large difference in Larmor frequencies between proton and carbon nuclei or via an INEPT sequence from ${ }^{1} \mathrm{H}$ to ${ }^{13} \mathrm{C}$ after hyperpolarization at $50 \mathrm{Gs}$.

The clinically used drugs isoniazid and pyrazinamide were probed by Zeng et al. using SABRE with $[\operatorname{Ir}(C O D)(I M e s) C l]$ as precatalyst in order to determine the parameters for their optimal hyperpolarization [49]. To that end, the duration of $p \mathrm{H}_{2}$ application, the polarization field and the temperature were varied for a catalyst-to-substrate ratio of 1:10 in methanol- $d_{4}$ and methanol- $h_{4}$ as well as the clinically more appropriate solvents ethanol and DMSO. The exposure time was found to be solvent specific and dependent on the viscosity and the solubility of $H_{2}$. For pyrazinamide, optimal enhancement in methanol- $d_{4}$ and methanol- $h_{4}$ was achieved at temperatures of $46.1^{\circ} \mathrm{C}$ ( 1400 -fold and $~ 800$-fold, respectively), while for ethanol and DMSO the optimal temperature was $37.5^{\circ} \mathrm{C}$. For all solvents, highest levels of 
enhancement were achieved at a polarization field of $65 \mathrm{Gs}$. Compared to methanol- $d_{4}$, enhancement values for methanol- $h_{4}$ were reduced to approx. $50 \%$, for ethanol to $25 \%$ and for DMSO to $3 \%$. For isoniazid, the optimal polarization field was identical and highest levels of polarization were for all solvents achieved at $46.1{ }^{\circ} \mathrm{C}$. Compared to methanol- $d_{4}$, the level of enhancement was reduced to $60 \%, 50 \%$ and $15 \%$ for methanol- $h_{4}$, ethanol and DMSO, respectively. With optimized parameters, the ratio of enhancement between pyrazinamide and isoniazid was 1400:230 in methanol- $d_{4}$.

A further step towards clinical application of SABRE was taken by Mewis et al., who studied the deactivation of the active catalyst species after performing the hyperpolarization step [50]. To do so, the authors performed SABRE for the analyte nicotinamide using $[\operatorname{Ir}(\mathrm{COD})(\mathrm{IMes}) \mathrm{Cl}]$ as the precatalyst and subsequently added 2,2'-bipyridine to the sample solution. Examination using NMR revealed SABRE enhancement for the initial experiment, yet a repolarization of the substrate was not possible. ${ }^{1} \mathrm{H}$ NMR spectra showed a newly formed dominant hydride resonance, corresponding to the complex $\left[\operatorname{Ir}(\mathrm{H})_{2}(\operatorname{IMes})(\text { bipy })(\text { nico })\right]^{+}$(bipy $=2,2^{\prime}$-bipyridine, nico $=$ nicotinamide), in which the bipyridine ligand occupies the positions trans to the hydrides. Consequentially, hydride exchange in this complex is effectively inhibited, as the exchange pathway includes dissociation of an equatorial ligand, which does not occur for the chelating bipyridine molecule, resulting in deactivation of the polarization transfer complex. The deactivation further causes an elongation of the observed substrate $T_{1}$ times, which are shortened in the presence of the active complex and are restored to their free form values after addition of the chelating ligand. This allows for examination of the hyperpolarized samples over a significantly expanded time frame compared to unmodified SABRE experiments, permitting more time-consuming experimental procedures, such as in vivo experiments, for which the sample must first be injected into the organism of interest. This procedure was successfully transferred to the substrate pyridine and the chelate ligand 1,10-phenanthroline. Reactivation of the deactivated complex using UV-irradiation proved unsuccessful.

Another in-depth study on individual substrates was conducted by Appleby et al., who investigated pyridazine and phthalazine, both of which exhibit two adjacent nitrogen atoms in their aromatic ring structures [31]. The analytes were found to undergo intramolecular haptotropic shift, i.e. change of the coordination motif from one to the other donor atom, when coordinated to a metal ion. In solution, the complexes $[\operatorname{Ir}(\operatorname{COD})(\operatorname{IMes})(\mathrm{L})]^{+}(\mathrm{L}=$ pyridazine, phthalazine) were formed, which were transformed into $\left[\operatorname{Ir}(\mathrm{H})_{2}(\mathrm{COD})(\mathrm{IMes})(\mathrm{L})\right]^{+}$ after application of $\mathrm{H}_{2}$ at $240 \mathrm{~K}$. PHIP activity was observed for the hydrides in these complexes. Upon warming to room temperature, the complexes reacted swiftly under release of cyclooctane to form the active SABRE catalyst species $\left[\operatorname{Ir}(\mathrm{H})_{2}(\mathrm{IMes})(\mathrm{L})_{3}\right]^{+}$. In-depth analyses of all observed I $\mathrm{r}-\mathrm{L}$ complexes, including ligand and hydride exchange are given by the authors. Both analytes exhibited high susceptibility to the SABRE protocol and achieved higher levels of enhancement at higher temperatures. MRI under analytical conditions using 
these substrates was highly feasible and preliminary results indicated excellent rates of polarization transfer in ethanol- $d_{6}$ solutions.

The behavior of SABRE for highly diluted samples was investigated by Eshuis et al. [51]. The authors examined enhancement of pyridine using $\left[\operatorname{Ir}(\mathrm{H})_{2}(\mathrm{IMes})(\mathrm{py})_{3}\right]^{+}$as the polarization transfer catalyst at a fixed catalyst-to-substrate ratio of 1:12.5 for substrate concentrations ranging from $10 \mathrm{mM}$ to $25 \mu \mathrm{M}$. For analyte concentrations of below $2.5 \mathrm{mM}$, a rapid decrease in SABRE activity was observed, owing to dissociation of the active polarization transfer catalyst. Due to the low substrate concentration, dissociated analyte molecules are not effectively replaced by free substrates from the sample solution, but vacant coordination sites are presumably occupied by solvent molecules. Therefore, at sub-mM concentrations, the major percentage of pyridine is free in solution and formation of the active catalyst species is inhibited, resulting in a loss of polarization transfer activity. To counter this effect, the authors employed 1-methyl-1,2,3-triazole as a co-substrate in order to increase the total substrate concentration and to facilitate the formation of active polarization transfer catalysts. Using this method, micromolar concentrations of pyridine were detectable and the detection limit was estimated to be $<1 \mu \mathrm{M}$. In this concentration regime, the SNR was found to be linearly dependent on the concentration of the analyte, provided that the concentration of the co-substrate was constant and exceeded that of the analyte by a wide margin.

The same research group employed this method by examining a substrate mixture containing $8.8 \mu \mathrm{M}$ nicotinamide as the analyte as well as 15 additional compounds as cosubstrates with a combined substrate concentration of $6 \mathrm{mM}$ [52]. The analyte concentration was then stepwise increased over a number of SABRE experiments. The initial concentration could subsequently be determined within experimental error by plotting the observed signal intensity against the added analyte amount and extrapolating the abscissa intercept. The concentrations of pyrazine, isoxazole and quinazoline were examined in the same manner.

In a similar approach, this research group examined the complexes formed from an initial iridium compound when exposed to a mixture of 13 SABRE active compounds by analyzing the PHIP-derived enhancement of the hydride resonances in the complexes. The researchers exploited the fact that due to the variety of substrates virtually only asymmetric iridium complexes of the form $\left[\operatorname{Ir}(\mathrm{H})_{2}(\mathrm{IMes})\left(\text { substrate)(substrate')(substrate }{ }^{\prime \prime}\right)\right]^{+}$are generated, which give rise to strongly enhanced hydride resonances when exposed to $\mathrm{pH}_{2}$ [53]. The substrates were employed at concentrations ranging from $250 \mathrm{nM}$ to $2 \mu \mathrm{M}$ at a metal complex concentration of $2 \mathrm{mM}$ and a co-substrate concentration of $30 \mathrm{mM}$. Experiments were performed under PASADENA conditions $\left(\mathrm{pH}_{2}\right.$ exposure and signal detection at highfield) and polarization transfer from the hydrides to analyte ortho protons via long range scalar coupling was achieved using a shaped pulse based ${ }^{1} \mathrm{H}-{ }^{1} \mathrm{H} 2 \mathrm{D}$ pulse sequence. The resulting spectrum was acquired in $23 \mathrm{~min}$ and the achieved signal separation allowed clear assignment of all substrate molecules. Also, for this approach, the signal intensity is linearly 
dependent on the analyte concentration, allowing for a titration in order to determine the initial analyte concentration using the $2 \mathrm{D}$ resonance integrals.

In a very recent 2016 study, Hermkens et al. examined a coffee extract solution using a modified version of the afore described high-field co-substrate protocol [54]. The modification consisted of application of a slightly different 2D pulse sequence, in which the polarization was not only transferred from the hydride nuclei to the substrate protons but was also transferred back and detected on the hydride resonances. This served the purpose of reducing the number of undesired resonances in the spectra, as no signal of free analyte molecules or symmetrical complexes was recorded. Using the titration method, the concentrations of several heterocyclic compounds in the coffee extract were quantitatively determined in satisfactory agreement with tabulated values.

Daniele et al. reported on a minor modification of the co-substrate approach, in which the authors employed pyridine- $d_{5}$ in order to analyze a mixture of five substrates at sub-millimolar concentrations [55]. The deuterated co-substrate was used in order to prevent a signal overlap with resonances of interest and proved to increase the enhancements observed on the targeted substrates. In a one-scan ultrafast 2D COSY experiment using $0.13 \mathrm{mM}[\operatorname{Ir}(\mathrm{COD})(\mathrm{IMes}) \mathrm{Cl}], 10 \mathrm{mM}$ pyridine- $d_{5}$ and $500 \mu \mathrm{M}$ of the individual analytes, a good quality spectrum was recorded after SABRE at 70 Gs and 4 bar $\mathrm{pH}_{2}$ (51\%), which allowed assignment of all substrates except for the SABRE-unsuitable 2-ethylpyridine.

Hyperpolarization of non-heterocyclic substrates was investigated by Mewis et al., who examined acetonitrile and other aromatic and nonaromatic nitrile-containing substrates [56]. Notably, experiments performed after the initial activation step of [ $\operatorname{lr}(\mathrm{COD})(\mathrm{IMes}) \mathrm{Cl}]$ using parahydrogen produced lower enhancement levels than the initial experiment, indicating a poor polarization transfer activity of the typical $\left[\operatorname{Ir}(\mathrm{H})_{2}(\mathrm{IMes})(\mathrm{NCMe})_{3}\right]^{+}$complex for the analyte acetonitrile. Addition of small amounts of pyridine in a ratio of 20:3 resulted in the formation of the more active and thermodynamically favored complex $\left[\operatorname{Ir}(\mathrm{H})_{2}(\mathrm{IMes})(\mathrm{NCMe})(\mathrm{py})_{2}\right]^{+}$, which showed and maintained a higher level of polarization transfer to the target substrate, resulting in an enhancement factor of 20 at a polarization field of $80 \mathrm{Gs}$. Employment of pyridine- $d_{5}$ further increased the enhancement to 60 -fold, while addition of $\mathrm{PCy}_{3}$ to form a phosphine- and $\mathrm{NHC}$-containing catalyst decreased the observed signal intensity considerably. Hyperpolarization transfer to ${ }^{13} \mathrm{C}$ nuclei in acetonitrile was also achieved and the underlying mechanics were discussed thoroughly.

In an effort to maximize the detectable hyperpolarization on the solvent resonance, Moreno et al. examined various substrates under mildly acidic conditions in order to generate labile protons at the analyte donor atoms [57]. The authors employed two NHC carrying complexes in order to facilitate the polarization transfer from the parahydrogen nuclei. Under neutral conditions, a slight dependence of the solvent signal was observed in the NMR spectra when pyridine was employed as the analyte, yet the signal integrals remained 
lower than in the thermal references. When the sample was acidified using hydrochloric acid, however, significant enhancement of the solvent resonance was observed. It was postulated that this polarization stems from analyte molecules that are intermediately protonated after obtaining hyperpolarization at the polarization transfer catalyst. During this time, the temporary $\mathrm{NH}$ proton obtains magnetization due to an intramolecular redistribution mechanism across the scalar coupling network and is subsequently transferred onto a solvent molecule, where it is detected in a hyperpolarized state. A significant decrease in solvent hyperpolarization by employment of pyridine- $d_{5}$ backed this proposed mechanism.

\subsubsection{Polarization Transfer to Heteronuclei}

As a result of their inherently low natural abundance and sensitivity, hyperpolarization of heteronuclei like ${ }^{13} \mathrm{C},{ }^{15} \mathrm{~N}$ and - to a lower extent - ${ }^{31} \mathrm{P}$ is highly desirable in order to shorten experimental run times. However, SABRE polarization transfer to these nuclei is not as readily achieved as for proton spins and special precautions have to be taken to facilitate the hyperpolarization. This includes the utilization of so called $\mu$-metal cylinders, which are made from an alloy containing nickel, iron, copper and various other metals. This material exhibits the property of efficiently shielding the inside of the container from prevalent magnetic fields, so that the effective field can be reduced to levels well below the earth's field. The following studies of SABRE polarization transfer to heteronuclei have been reported:

Zhivonitko et al. examined the hyperpolarization of ${ }^{31} \mathrm{P}$ nuclei by analyzing the enhancement of $\mathrm{PPh}_{3}$ using the iridium complex $\left[\mathrm{Ir}(\mathrm{H})_{2}\left(\mathrm{PPh}_{3}\right)_{3} \mathrm{Cl}\right]$ [58]. The catalyst was created in situ from a $6 \mathrm{mM}[\operatorname{Ir}(\mathrm{COD}) \mathrm{Cl}]_{2}$ solution by addition of $\mathrm{PPh}_{3}$ in a ratio of $1: 5$ and subsequent activation using $p \mathrm{H}_{2}$. Polarization was achieved by application of parahydrogen at a field of $\sim 10 \mathrm{mGs}$ inside a $\mu$-metal box. At room temperature, no observable enhancement was detected. At 60 and $80^{\circ} \mathrm{C}$, however, the signal intensity of the free phosphine resonance was increased by factors of about 120 and 260, respectively. The complex-coordinated phosphines were also polarized. Application to MRI after polarization at $80^{\circ} \mathrm{C}$ allowed well-resolved image acquisition in a single ${ }^{31} \mathrm{P}$ scan. Further reported findings are the increase of ligand exchange rates at higher temperatures and highest efficiency of polarization transfer to ${ }^{31} \mathrm{P}$ at lowest magnetic field strengths. Proton enhancement of free and trans-bound $\mathrm{PPh}_{3}$ was also observed and concluded to originate directly from the former $\mathrm{pH}_{2}$ nuclei rather than from magnetization redistribution after polarization of the ${ }^{31} \mathrm{P}$ nuclei. 


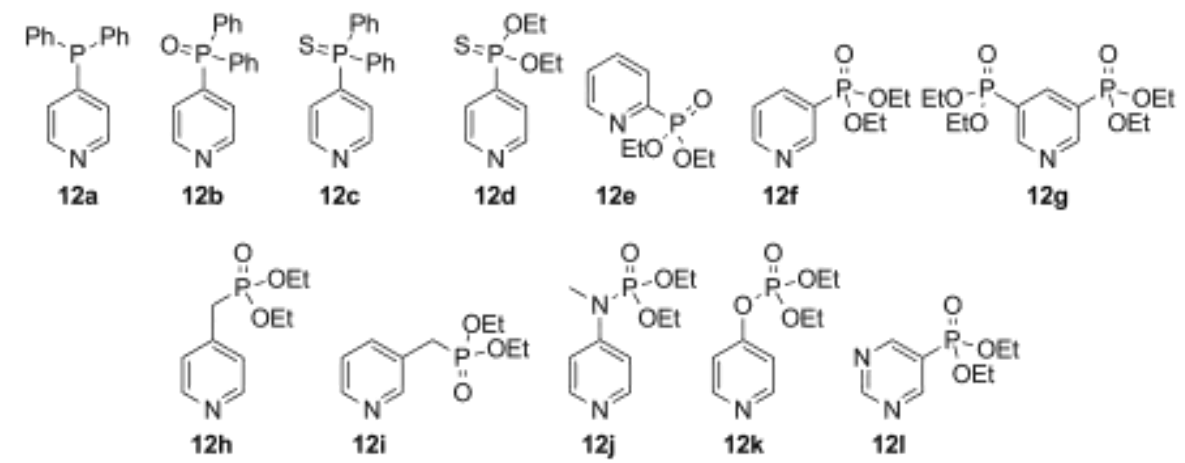

Figure 5: Chemical structures of pyridine derivatives, into which phosphorus functionalities were introduced to be examined in ref. [59].

In a different approach to the examination of ${ }^{31} \mathrm{P}$ spins using SABRE, Burns et al. synthesized several pyridine derivatives substituted with phosphorus functionalities and probed their interaction with parahydrogen via the $\left[\operatorname{Ir}(\mathrm{H})_{2}(\mathrm{IMes})(\text { substrate })_{3}\right]^{+}$polarization transfer catalyst [59]. The initial group of analytes consisted of four 4-substituted pyridines bearing a phosphine (12a, Figure 5), phosphine oxide (12b), phosphine sulfide (12c) or phosphonate ester (12d) functionality. Using a $5 \mathrm{mM}$ substrate solution, a catalyst loading of $17 \%$ and a polarization field of $0.5 \mathrm{Gs}$, the phosphorus resonance of phosphine sulfide was only weakly enhanced by a factor of 6 . For the phosphine, the phosphine oxide and the phosphonate ester, however, the corresponding resonances were reported to be enhanced by factors of 57, 32 and 545, respectively. Examination of the matching analytes $\mathrm{PPh}_{3}, \mathrm{OPPh}_{3}, \mathrm{PPh}(\mathrm{OEt})_{2}$ showed no enhancement at $298 \mathrm{~K}$, proving the importance of the pyridyl moiety for the polarization transfer. Since the phosphonate ester showed highest susceptibility, further diethyl pyridylphosphonate esters (12e-k) were synthesized, of which the 3-substituted (12f) and the 3,5-disubstituted $(\mathbf{1 2} \mathrm{g})$ versions exhibited highest levels of polarization with ${ }^{31} \mathrm{P}$ enhancement factors of 336 and 860 , respectively. Deuteration of the phosphonate ethoxy functions resulted in further increased levels of ${ }^{31} \mathrm{P}$ enhancement up to a factor of 3588 for the disubstituted version. Incorporation of various spacer groups between the heterocycle and the phosphorus $(\mathbf{1 2} \mathbf{h}-\mathbf{k})$ resulted in significantly reduced levels of ${ }^{31} \mathrm{P}$ polarization. The observed ${ }^{31} \mathrm{P}$ resonances exhibited a high antiphase character, which was also evident for the ${ }^{1} \mathrm{H}$ resonance of the neighboring ring protons and which was caused by formation of proton-phosphorus two spin order terms. An increase in the polarization transfer field to $45 \mathrm{Gs}$ increased the relative in-phase contribution, as the relative proportions are shifted from two spin to single spin components, while maintaining the level of achieved enhancements. Increasing the polarization field strength even more resulted in a reduction of observable signal intensity, yet even greater in-phase contributions were detected. Performing the polarization step in a $\mu$-metal box yielded strictly in-phase ${ }^{31} \mathrm{P}$ resonances at the cost of signal intensity reduction by one order of magnitude. Additionally, one-shot ${ }^{31} \mathrm{P}$ MRI was accomplished using the synthesized pyridylphosphonate esters.

In order to achieve hyperpolarization of ${ }^{15} \mathrm{~N}$ nuclei, Theis et al. performed the SABRE polarization step inside a magnetic field-cancelling $\mu$-metal box at an effective field on the 
order of $<10 \mathrm{mGs}$ [60]. The SABRE-SHEATH (SABRE in SHield Enables Alignment Transfer to Heteronuclei) termed protocol achieved highly efficient spontaneous polarization transfer to ${ }^{15} \mathrm{~N}$-labelled pyridine, giving signal enhancement factors on the order of 30000 for a $4 \mathrm{mM}$ sample compared to the thermal reference at $94 \mathrm{kGs}$, corresponding to a level of polarization of $\sim 10 \%$. In contrast to spectra recorded using ${ }^{1} \mathrm{H}$ optimized experimental parameters, i.e., polarization field in the range of 10 to $250 \mathrm{Gs}$, the resonances were observed as in-phase, emissive signals. Increasing the overall concentration yielded higher signal intensities, yet lower enhancement factors, which is attributed to the finite amount of $\mathrm{pH}_{2}$ derived polarization, a change in the ligand exchange rates and increased relaxation rates due to a higher spin density. Signal enhancement of the catalyst-bound analytes was also recorded. Application to nicotinamide yielded ${ }^{15} \mathrm{~N}$ enhancement levels of 20000 for a $48 \mathrm{mM}$ solution.

This polarization protocol was later utilized to facilitate the hyperpolarization of solvent-free analyte samples [61]. When neat natural abundance pyridine was examined using a $90 \mathrm{mM}$ catalyst concentration, enhancements on the order of -2900 were observed for the ${ }^{15} \mathrm{~N}$ resonance, while ${ }^{1} \mathrm{H}$ enhancement of the same sample after polarization at $60 \mathrm{Gs}$ was only by a factor $4 .{ }^{15} \mathrm{~N}$-labelled pyridine experienced enhancement only by a factor of 33 , attributed to insufficient $p \mathrm{H}_{2}$ availability, as the influx on fresh parahydrogen, which was applied to the solution via a small diameter tube, proved to be a limiting factor. This was further elucidated as the signal intensity depended linearly on the applied $\mathrm{pH}_{2}$ flow rate and the absolute pressure had no discernable effect, indicating that the para-to-ortho conversion was rapid compared to the replenishing of $\mathrm{pH}_{2}$. Due to the highly superior enhancement of the natural abundance pyridine, the authors further postulated that coordination of a ${ }^{14} \mathrm{~N}$-pyridine molecule to the catalyst complex does not deplete the polarization stored in the hydride nuclei, presumably because fast quadrupolar relaxation of theses nuclei effectively decouples them from the former parahydrogen spins. Employment of pyridine- $d_{5}$ as the analyte resulted in lower observed ${ }^{15} \mathrm{~N}$ enhancement, as the quadrupolar ${ }^{2} \mathrm{H}$ nuclei significantly reduced the ${ }^{15} \mathrm{~N} \mathrm{~T}_{1}$ times at fields $<10 \mathrm{mGs}$. Furthermore, polarization transfer to these nuclei is also achieved at near-zero fields, reducing the amount of available polarization for the ${ }^{15} \mathrm{~N}$ nuclei. This protocol was further employed to successfully enhance pure picolines and lutidines and it was found that 2-methylsubstituted pyridine derivatives are not susceptible to SABRE.

Just very recently, Shchepin et al. reported on the SABRE-targeted synthesis of nicotinamide with a selective ${ }^{15} \mathrm{~N}$-labelling in the heterocycle position using a modified Zincke reaction [62]. In combination with SABRE-SHEATH, excellent levels of hyperpolarization were detected on the incorporated ${ }^{15} \mathrm{~N}$ nucleus, corresponding to enhancement of up to 11000 -fold using $50 \%$ para-enriched $\mathrm{H}_{2}$ in natural abundance methanol. The mechanism is described as direct transfer via J-coupling from the polarized $\mathrm{H} 2$ to the $15 \mathrm{~N}$. In combination with the low toxicity of the analyte and clinically applicable solvents, the application of this substrate as an MRI contrast agent appears feasible. 
A further study on the transfer of SABRE derived polarization to ${ }^{15} \mathrm{~N}$ nuclei was conducted by Pravdivtsev et al., who compared the results achieved using three different experimental procedures and two different polarization transfer catalysts using $70 \mathrm{mM}{ }^{15} \mathrm{~N}$-labelled pyridine and $2 \mathrm{mM}$ catalyst concentrations [63]. The first protocol corresponds to SABRE-SHEATH, in which polarization is transferred directly from the $\mathrm{pH}_{2}$ derived hydrides to the ${ }^{15} \mathrm{~N}$ nuclei via strong scalar coupling. The ${ }^{15} \mathrm{~N}$ signals were observed as emissive in-phase multiplets that can be collapsed by selective or broadband ${ }^{1} \mathrm{H}$ decoupling. Coordinated analytes also exhibited enhancement, which was one order of magnitude smaller for the axially coordinated pyridines $(\sim 200)$ compared to the free $(\sim 2500)$ and equatorially bound ( 2250) molecules. Using pyridine- $d_{5}$, multiple ${ }^{15} \mathrm{~N}$ resonances of free pyridine were observed, corresponding to different partial protonation patterns. In the second protocol, the polarization step was performed at close to earth's field, where the strong coupling prerequisite is no longer given between ${ }^{1} \mathrm{H}$ and ${ }^{15} \mathrm{~N}$ spins. Under these conditions, no net polarization is transferred, but individual lines in antiphase multiplets are enhanced by a significant margin ( 1600) for free pyridine. Application of proton decoupling results in cancellation of the lines with different phases, collapsing the signal. For the third protocol, the authors applied a previously published experimental design [64] to SABRE that was developed for the transfer of PHIP derived hyperpolarization to heteronuclei at high-field. In order to achieve this, the hydrogenation reaction (PHIP) or the replenishing of solvated $\mathrm{pH}_{2}$ (SABRE) is performed inside the NMR magnet and the $\mathrm{pH}_{2}$-derived polarization is subsequently transferred to the desired nucleus by application of two RF fields, $\mathrm{RF}_{H}$ and $\mathrm{RF}_{\text {het }} \mathrm{RF}_{\mathrm{H}}$ was adjusted to be resonant to the former $\mathrm{pH}_{2}$ nuclei at a constant amplitude, while $\mathrm{RF}_{\text {het }}$ was set to varied frequencies in the resonance range of the targeted heteronucleus and its amplitude linearly reduced to zero. By doing so, the spin system passed through the Hartmann-Hahn condition [65] and consequentially through a Level AntiCrossing (LAC), resulting in a strong coupling between the ${ }^{15} \mathrm{~N}$ and ${ }^{1} \mathrm{H}$ nuclei, facilitating direct polarization transfer between the individual spins. The FID was recorded after a $90^{\circ}$ excitation pulse on the heteronucleus channel. Using this sequence for SABRE, enhancement factors of 800 and 12000 were determined for free and equatorially coordinated pyridine, respectively, compared to thermal signals at $94 \mathrm{kGs}$. By varying the $\mathrm{RF}_{\text {het }}$ frequency, the polarization phase can be modulated. In addition, continuous hyperpolarization experiments were conducted using the third setup, in which the delay between individual experiments was varied. It was found that highest levels of enhancement were achieved when employing shorter delays, presumably due to more residual $\mathrm{pH}_{2}$ in the solution and different amounts of delivered $\mathrm{pH}_{2}$ due to more pronounced capillary effects for longer delays. It was further noticed that for this protocol, longer complex life times are beneficial, as the complex has to stay intact over the course of the pulse sequence to facilitate an efficient transfer of polarization.

\subsubsection{SABRE Polarization Transfer at High Field}

The sample transfer from the low-field to the high-field position inside the NMR magnet poses an undesired complication of the experimental procedure in SABRE experiments. 
However, at the high-field regime inside the NMR magnet, the requirements for a spontaneous polarization transfer via the scalar coupling network are no longer given, rendering the polarization step at low-field a necessity in the unmodified SABRE protocol. To overcome this, special experimental protocols have been developed, in which the low-field regimen is emulated by application of radio frequency irradiation:

Already prior to the publication of ref. [63], Pravdivtsev et al. as well as Theis et al. had reported on polarization transfer from $\mathrm{pH}_{2}$-hydride ligands to analyte nuclei achieved at the high-field regimen inside the NMR magnet. In the Pravdivtsev publications [66, 67], individual ${ }^{1} \mathrm{H}$ resonances were selectively enhanced by application of continuous wave (CW) RF irradiation. After exposure of the sample to parahydrogen, an RF field carefully adjusted to the center frequency between the hydride and the target resonances modulated by a small offset is applied on the ${ }^{1} \mathrm{H}$ channel to fulfill the Hartmann-Hahn condition and to create a Level Anti-Crossing. Thereby, the nuclear spin state populations of these nuclei are mixed, facilitating a transfer of polarization to the substrate nuclei in the form of $x$-magnetization. An in-depth theoretical description of the occurring spin operators is given in ref. [67]. Signal acquisition is performed directly after the CW irradiation is stopped without a further application of pulses. By shifting the irradiation frequency, different target resonances can be selected and by varying the offset from the center frequency, the target signals can be modulated to either appear in absorptive or emissive phase. Polarization of the hydride ligands as well as of free $\mathrm{H}_{2}$ is observed, which exhibits the opposite phase relative to the substrate resonance. Enhancement by two orders of magnitude was achieved using this experimental protocol.

The publication by Theis et al. reports on a very similar protocol, but focuses on the transfer of polarization to ${ }^{15} \mathrm{~N}$ spins [68]. The authors employed a pulse sequence consisting of $\mathrm{CW}$ irradiation for selective excitation of catalyst-bound ${ }^{15} \mathrm{~N}$ resonances, although transfer to other nuclei is also feasible. The prerequisites for efficient transfer are the symmetry of the metal complex in respect to the hydride ligands and the amplitude of the $\mathrm{CW}$ irradiation matching the sum of the hydride-hydride and ${ }^{15} \mathrm{~N}-{ }^{15} \mathrm{~N}$ scalar couplings, for which $J_{\mathrm{HH}}+J_{\mathrm{NN}}=12 \mathrm{~Hz}$ was estimated. By application of the $\mathrm{CW}$ irradiation, polarization is transferred to the ${ }^{15} \mathrm{~N}$ nuclei during the lifetime of the complex in the form of $x$-magnetization. To avoid dephasing of this magnetization after dissociation of the complex, a selective $90^{\circ}$ pulse is applied to the nitrogen resonance of the coordinated ${ }^{15} \mathrm{~N}$ nuclei, which creates $z$-magnetization on the ligand spins. By repetition, high levels of ${ }^{15} \mathrm{~N}$ polarization can be accumulated on free analyte molecules, which can then be observed by a $90^{\circ}{ }^{15} \mathrm{~N}$ hard pulse. A full theoretical exposition is provided in the original publication. This modified high-field SABRE protocol was termed Low Irradiation Generation of High Tesla Signal Amplification By Reversible Exchange (LIGHT-SABRE).

\subsubsection{SABRE Detection at Low Field}

In low-field NMR ( $B_{0} \sim<250 \mathrm{Gs}$ ), the thermal magnetization due to the Boltzmann population difference of the Zeeman energy levels is by orders of magnitude lower than for 
high-field spectrometers and often additional means of sample polarization, e.g. SABRE, are required in order to observe sufficiently intense signals. Owed to the reduced precession frequencies in combination with the natural line width, the signal resolution is commonly rather poor, yet acquisition and operation of such low-field spectrometers is highly costeffective and low in maintenance. Application of low-field NMR is therefore highly convenient in cases where only the presence or absence of a single compound is expected and spectral resolution is of minor importance.

Gong et al. were the first to apply the SABRE technique in low-field NMR experiments $\left(B_{0}=39 \mathrm{Gs}\right.$ ) and compared the attained signal intensities to those recorded using either SPINOE or thermal prepolarization in a $20 \mathrm{kGs}$ Halbach magnet as a means of generating observable magnetization [69]. SABRE proved to be highly effective for detection of trace amounts of substrate and the authors estimated a level-of-detection limit of only $12 \mathrm{nmol}$ in $0.4 \mathrm{~mL}$ sample volume. The level of enhancement was further determined to be proportional to the applied pressure of $\mathrm{pH}_{2}$, as the amount of dissolved hydrogen poses a limitation to the number of molecules to be enhanced. For substrate concentrations below the concentration of dissolved $\mathrm{pH}_{2}$, the signal intensity was found to be linearly dependent on the substrate concentration, until [substrate] $=\left[p \mathrm{H}_{2}\right]$. After that point, the signal intensity remained constant for a range of substrate concentrations, as the amount of dissolved $p \mathrm{H}_{2}$ limited the total amount of polarization to be transferred. For highly concentrated solutions, the SNR decreased because of an increase in viscosity and spin density in solution, leading to higher relaxation rates.

Also working in the low-field regime, Glöggler et al. examined amino acids and di- and tripeptides for their susceptibility to SABRE using Crabtree's catalyst $\left[\operatorname{Ir}(\mathrm{COD})\left(\mathrm{PCy_{3 }}\right)(\mathrm{py})\right] \mathrm{PF}_{6}$ and a spectrometer with an adjustable $B_{0}$ field of either $39 \mathrm{Gs}$ or 2.5 Gs [70]. The examined substrate amounts were in the $\mathrm{nM}$ range and thereby well under the detection limit at thermal polarization, yet successful detection using parahydrogen was reported for all proteinogenic amino acids despite their low solubility in the solvent methanol- $d_{4}$. Furthermore, hyperpolarization of two short peptides was observed, indicating a possible applicability of SABRE to proteins. As individual resonances were not resolved due to the natural line width and the low precession frequencies in low-field NMR spectrometer, the origin of the detected signal cannot be unequivocally attributed to the examined amino acids, but it can only be said that a resonance was detected. Possible other sources are the solvent, hydrogen gas or the isotope-mixed hydrogen gas HD, which was created by HD-exchange between solvent and hydride ligands and observed to be hyperpolarized in the process, presumably by hyperpolarization of the solvent- ${ }^{2} \mathrm{H}$ and subsequent recombination to give polarized HD. Reference spectra were recorded in high-field spectrometers to ensure that no modification of the substrates had occurred.

Contrary to this publication, however, Trantzschel et al. reported that no signal enhancement was observable for the analytes lysine, glycine or $\gamma$-aminobutyric acid in their efforts to detect hyperpolarized amino acids using SABRE in combination with high-field 
spectrometers [71]. They thus failed to reproduce the experimental results of Glöggler et al. [70].

Staying on the topic of low-field NMR, Borowiak et al. developed a cost-effective and easy to build do-it-yourself low-field spectrometer, designed to especially fulfill the requirements for SABRE and PASADENA experiments [72]. A highly homogenous magnetic field was generated by an adjustable, 2-layered solenoid coil with a third layer of compensation windings at the outer ends. Power to the electrical parts was supplied by a battery-driven current controller in an approach to reduce the electrical noise of the signal. In order to demonstrate the functionality of the device, a $303 \mathrm{mM}$ pyridine solution was polarized using [ $\operatorname{lr}(\mathrm{COD})(\mathrm{IMes}) \mathrm{Cl}]$ at $56 \mathrm{Gs}$ with the SABRE procedure and exhibited a SNR on the order of $10^{4}$, while no signal was observable at thermal equilibrium.

This setup was later employed by Hövener et al. to examine the applicability of SABRE to the more clinically appropriate solvents ethanol and 9:1 $\mathrm{H}_{2} \mathrm{O}$-ethanol in order to avoid the toxicity of the regularly employed methanol [28]. Results for methanol obtained in situ at an identical polarization and detection field of $54 \mathrm{Gs}$ showed an estimated signal enhancement for methanol in the range of $320 \times 10^{3}$, corresponding to a polarization level of approx. $0.6 \%$. For pure ethanol and the $\mathrm{H}_{2} \mathrm{O}$ /ethanol mixture, respectable, yet lower polarization levels of 0.2 and $0.02 \%$ were achieved, respectively, corresponding to an enhancement on the order of $10^{4}$ to $10^{5}$. As individual resonances were not resolved in the low-field setup, control experiments were conducted at high-field $(7 \mathrm{~T})$ to exclude signal cancellation caused by phase variations. Here, a maximum enhancement for pyridine using the $[\operatorname{Ir}(\mathrm{COD})(\mathrm{IMes}) \mathrm{Cl}]$ precatalyst was discovered at polarization fields between 40 and $70 \mathrm{Gs}$, where the three pyridine resonances are in phase and therefore do not cancel each other out. To further exclude an effect of the sample transfer from low to high-field on the signal intensities, the experiments were repeated using a field cycling setup, in which the sample was polarized at low-fields between 5 and $227.5 \mathrm{Gs}$, and the resulting magnetization was detected at earth's field. Highest signal intensities for the solvents methanol and ethanol were found at polarization field comparable to those determined in the high-field experiments. Furthermore, single scan MRIs of 3,4,5-trideuteriopyridine $\left({ }^{1} \mathrm{H}\right)$ and carbonyl- ${ }^{13} \mathrm{C}$-labelled nicotinamide $\left({ }^{13} \mathrm{C}\right.$ ) were reported under analytical conditions (methanol, substrate, catalyst in an analytical tube).

The same low-field setup was further used in order to achieve continuous polarization of pyridine using SABRE in a low-field NMR setting and to subsequently apply the continuous hyperpolarization setup to low-field MRI, for which the polarization step was performed at a field of $65 \mathrm{Gs}$, while signal acquisition was performed at Earth field [73]. Constant levels of hyperpolarization were achieved over several hundred seconds, with the level of enhancement depending on the recovery time in between experiments. In the course of the study, hyperpolarization experiments in human blood were also successfully conducted. 
Further studies in the low-field regime were conducted by Barskiy et al., who examined the hyperpolarization of pyridine in in situ and ex situ experiments [74]. In situ experiments, in which polarization and detection were performed at $475 \mathrm{Gs}$, yielded enhanced resonances for both pyridine and the hydride ligands, while the ex situ protocol, in which the polarization was performed at $57.5 \mathrm{Gs}$, facilitated signal enhancements only on the analyte resonances. Due to the large chemical shift difference of $31.5 \mathrm{ppm}$, the resonances of hydrides and ligand were separated, which is uncommon for low-field NMR. Additionally, in situ experiments were conducted at $57.5 \mathrm{Gs}$, which allowed for continuous hyperpolarization and time-resolved monitoring of the polarization level using small tip angles. Similarly, in situ and ex situ MRI experiments were performed. Using the in situ protocol, real time snapshots of the sample were acquired, which allows conclusions to be drawn about spatial reaction dynamics.

Reducing the field strength even more, Theis et al. reported on the implementation of the SABRE technique to their zero-field NMR spectrometer, which employs an all-optical rubidium magnetometer for detection [75]. The sample was shielded from the earth's magnetic field using a $\mu$-metal shield and a DC magnetic field pulse was used to unlock the $J$-coupling information stored in heteronuclear scalar spin order. The observed ${ }^{15} \mathrm{~N}$ spectra show no chemical shifts, as no magnetic field is present at the sample location but give information on the scalar coupling constants present in the analyte. Using $S A B R E,{ }^{15} \mathrm{~N}$ labelled pyridine at a concentration of $40 \mathrm{mM}$ in $250 \mu \mathrm{L}$ sample volume was detectable in a single scan and the intensities of the recorded spectra were increased by four orders of magnitude compared to samples thermally prepolarized at $16 \mathrm{kGs}$. In-phase magnetization was enhanced.

\subsubsection{Heterogeneous SABRE Catalysts}

In clinical applications, where a hyperpolarized substrate may be employed as a contrast agent in vivo, separation of the solution from the possibly toxic catalyst is indicated. A feasible approach is given by attaching the catalyst complex to solid particles, which can be readily separated from the solution by means of filtration. The following efforts to achieve this goal have been reported:

Shi et al. reported on the first observation of heterogeneous SABRE (HET-SABRE) [76]. In order to generate the solid-phase bound catalyst, the complex [Ir(COD)(IMes)Cl] was treated with $\mathrm{AgPF}_{6}$ in THF under inert gas in order to remove the chloride ligand from the complex and to subsequently facilitate the coordination of a polymer-bound 4-dimethylaminopyridine moiety. $200 \mu \mathrm{L}$ of a $150 \mathrm{mM}$ pyridine solution were examined using approx. $1.5 \mu \mathrm{mol}$ of the solid-phase bound catalyst at a $\mathrm{pH}_{2}(64 \%)$ pressure of $3.2 \mathrm{bar}$ and a polarization field of $\sim 100 \mathrm{Gs}$. Emissive signals for the pyridine ${ }^{1} \mathrm{H}$ resonances verified the SABRE activity of the complex, achieving an estimated signal enhancement of 5.2, 4.1 and 2.7 for the ortho, meta and para resonances, respectively. Furthermore, no signals for coordinated substrate molecules were observable, as the polymer beads quickly precipitated on the bottom of the tube and therefore were outside of the NMR active sample volume. 
Separation of the solid particles and subsequent control experiments using only the supernatant showed no evidence for SABRE activity, proving that the SABRE active compound is attached to the solid particles.

In order to increase the surface-to-volume ratio of the HET-SABRE catalyst, the same group reported on the synthesis of two additional heterogeneous catalysts with a similar design [77]. The first of these catalysts consists of $\mathrm{TiO}_{2}$ nanoparticles, that were coated with poly(methacrylic acid) (PMAA) and functionalized using aminopyridine. Formation of the precatalyst species was achieved by preactivation using $\mathrm{AgPF}_{6}$ and coordination of the pyridine moiety to the iridium ion. In the second catalyst species, the solid-bound pyridine moiety was supplied by polyvinylpyridine (PVP) and formation of the precatalyst species was achieved in an identical fashion. Using these catalysts, enhancement levels of 7 (PVP) and 40 $\left(\mathrm{TiO}_{2} / \mathrm{PMAA}\right)$ were achieved for pyridine, surpassing the results for the first-generation catalyst. Again, the catalysts showed no signs of leaching. No high-field SABRE effects were observable using the heterogeneous catalysts. Recycling of the catalysts by separation from the liquid, drying and re-employment in a new solution was found to be feasible.

\subsubsection{Theoretical Descriptions and Computational Analyses}

In order to understand the underlying mechanics of the SABRE technique, various recent studies have focused on illuminating the theoretical aspects on which the spontaneous transfer of the parahydrogen-derived hyperpolarization is based:

In addition to the discovery of Level Anti-Crossings as the primary cause of efficient polarization transfer at low-field [37], which also plays an important role in other hyperpolarization methods [78], Pravdivtsev et al. studied the effect of field cycling on nonequilibrium spin polarizations [79] and gave an in-depth description of the SABRE field dependence [80]. In the latter publication, the authors also described the effect of different polarization fields on the resonance of observed orthohydrogen and touched on the field of high-field SABRE. The same group furthermore developed an analytical model for the SABRE process that combines a number of experimental parameters and their effect on the signal enhancement into a single equation [81], which serves to assess the parameters in need for optimization in order to achieve highest levels of polarization. In collaboration with further researchers, the group also provided a thorough quantitative analysis of the SABRE effect [82], taking into account the nuclear spin interactions and their field dependence as well as chemical exchange mechanisms.

To increase the accuracy of theoretical calculations of the SABRE effect, Eshuis et al. determined the long range ${ }^{4} \mathrm{~J}$ and ${ }^{5} \mathrm{~J}$ scalar coupling constants between the hydride ligands and the substrate protons in active polarization transfer complexes [83]. To do so, the authors exploited the observable PHIP enhancement of the hydride ligands in unsymmetrical iridium complexes obtained from the exposure of $[\operatorname{Ir}(\mathrm{NHC})(\mathrm{COD}) \mathrm{Cl}](\mathrm{NHC}=\mathrm{IMes}, \mathrm{SIMes}, \mathrm{IPr})$ to a combination of substrate molecules and $\mathrm{pH}_{2}$. The parahydrogen-derived magnetization was transferred back and forth between the hydrides and the substrate ligands using a 
Selective Excitation of Polarization using PASADENA (SEPP) based pulse sequence. Fitting of the time-dependent signal intensity allowed for the extraction of the long-range coupling constants. For ortho protons in six-membered heterocycles, the ${ }^{4} \mathrm{~J}$ couplings were found to be in the range of 1.1 to $1.2 \mathrm{~Hz}$, while ${ }^{5} \mathrm{~J}$ couplings to meta protons of such substrates were only in the range of $0.4 \mathrm{~Hz}$. Interestingly, ${ }^{4} \mathrm{~J}$ scalar coupling constant between hydride ligands and proton spins in five-membered heterocycles were of considerably smaller magnitude compared to six-membered rings, giving values of $\sim 0.6 \mathrm{~Hz}$.

In order to quantify the efficiency of their continuous low-field hyperpolarization setup [73], Hövener et al. presented a computational analysis, which included LACs, polarization field dependence and lifetime of the active polarization transfer catalyst [84]. While certain key points of the theoretical predictions were reflected in the experimental outcome, the employed model system required substantial modification in order to generate a more realistic depiction of the obtained experimental results.

Lastly, van Weerdenburg et al. determined a variety of complexes that are formed in the course of their low-concentration co-substrate method [51-53] using density functional theory (DFT) calculations and determined their contribution to the SABRE effect [85]. The computational results were compared to NMR spectra of SABRE samples and Extended X-ray Absorption Fine Structure (EXAFS) of frozen samples in order to validate the presence of the predicted complexes.

\subsection{Employed Catalyst Systems}

Two classes of iridium-based polarization transfer catalysts were utilized in this work to achieve the transfer of hyperpolarization from parahydrogen gas to the substrate molecules of interest. SABRE was first observed and described for a variety of phosphine-based complexes $[21,22,25]$ and consequently Crabtree's catalyst $[\operatorname{Ir}(\mathrm{COD})(\mathrm{PCy})(\mathrm{py})]^{+}$was used in various studies of the effect itself and of susceptible substrates [35, 69-71]. The efficiency of the polarization transfer catalyst was attributed to the electron-donating character of the phosphine ligand and its relative bulkiness due to the sterically demanding cyclohexyl substituents [25]. As $N$-heterocyclic carbene ligands exhibit an even greater electron-donating character, applicability of corresponding NHC-carrying iridium complexes to SABRE was probed [34, 40,44, 45]. Owing to the reported superior polarization transfer efficiency achieved especially by Ir-IMes complexes, NHC derived catalyst systems were utilized in the majority of studies up to this date.

\subsubsection{Crabtree's Catalyst}

Crabtree's catalyst is the name given to the highly effective hydrogenation precatalyst complex $\left[\operatorname{Ir}(\mathrm{COD})\left(\mathrm{PCy}_{3}\right)(\mathrm{py})\right] \mathrm{PF}_{6}$ (Figure 6) that was developed by Robert $\mathrm{H}$. Crabtree et al. in the late 1970s and reported on in studies on iridium complexes employing tertiary phosphines $[23,24]$. The complexes were designed as analogs to previously studied rhodium complexes [86] that exhibited high hydrogenation efficiency in polar, coordinating substrates such as ethanol or tetrahydrofuran, where iridium compounds proved to be disadvantaged 
[87]. In non-coordinating solvents, e.g., dichloromethane, however, the iridium complexes exhibited the highest hydrogenation potential observed up until that point and were able to transfer $\mathrm{H}_{2}$ into mono-, di-, tri- and tetra-substituted olefins.

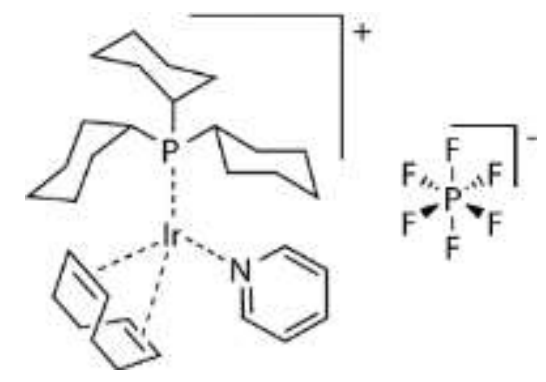

Figure 6: Chemical structure of Crabtree's catalyst $\left[\operatorname{Ir}(\mathrm{COD})\left(\mathrm{PC}_{3}\right)(\mathrm{py})\right] \mathrm{PF} 6$.

When exposed to hydrogen gas, the tetragonal $\operatorname{Ir}(\mathrm{I})$ complex reductively adds an $\mathrm{H}_{2}$ molecule is transformed into the active hexagonal $\operatorname{Ir}(\mathrm{III})$ hydrogenation catalyst $\left.\left[\mathrm{IrH}_{2}\left(\mathrm{PCy}_{3}\right) \text { (substrate) }\right)_{2}(\mathrm{py})\right]^{+}$, which is stabilized by the presence of the substrate. Upon consumption of the substrate, the complex was found to irreversible deactivate by di- and trimerization. This issue was later addressed by ligand modification [88] and lately chirally modified versions of the original complex have been employed in highly efficient asymmetric hydrogenation reactions [89].

The catalyst is, however, not powerful enough to reduce the $\pi$-bond system of aromatic substrates, so that such molecules containing a donor atom will only reversibly coordinate to the iridium complex and remain chemically unaltered. This is exploited in the SABRE protocol.

\subsubsection{N-Heterocyclic Carbenes}

This section is based on a review by Hopkinson et al. [90].

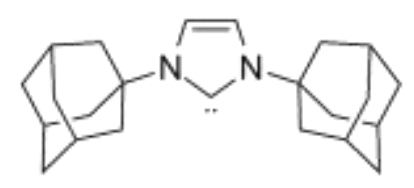

Figure 7: Chemical structure of 1,3-di(adamantyl)imidazole-2-ylidene.

Even though $\mathrm{N}$-heterocyclic carbenes had been present in chemical science beforehand, research interest increased immensely [90-94] after the synthesis of 1,3di(adamantyl)imidazole-2-ylidene (Figure 7), the first isolable carbene in a stabilizing heterocyclic environment, was reported by Arduengo et al. in 1991 [95]. In this substance class, the divalent carbene carbon is flanked by heteroatoms in a small - usually fivemembered - ring structure and exhibits only six valence electrons. The two electrons not located in a $\sigma$-bond exist as a lone pair in a formal $s p^{2}$-hybridized orbital and induce the characteristic $\sigma$-nucleophilic reactivity, while the remaining $p$-orbital remains unpopulated. Several factors contribute in order to stabilize this highly reactive species: 
The adjacent heteroatoms, i.e., most often nitrogen, yet oxygen and sulfur have also been reported, withdraw $\sigma$-electron-density from the carbon atom, leading to a reduced nucleophilicity of the carbene, while their $\pi$-donating character stabilizes the vacant $p$-orbital by mesomeric effects. An unsaturation in the backbone of the heterocycle further increases this stabilization by a partial aromatic effect. In addition, the five-membered ring structure forces the carbene carbon into a geometry which favors the described hybridization and the usually sterically demanding nitrogen substituents kinetically exacerbate a dimerization reaction. However, as can be seen from Figure 8 , not all of these factors need to be fulfilled, as a variety of different carbene classes have been reported that differ in certain aspects from the given description.

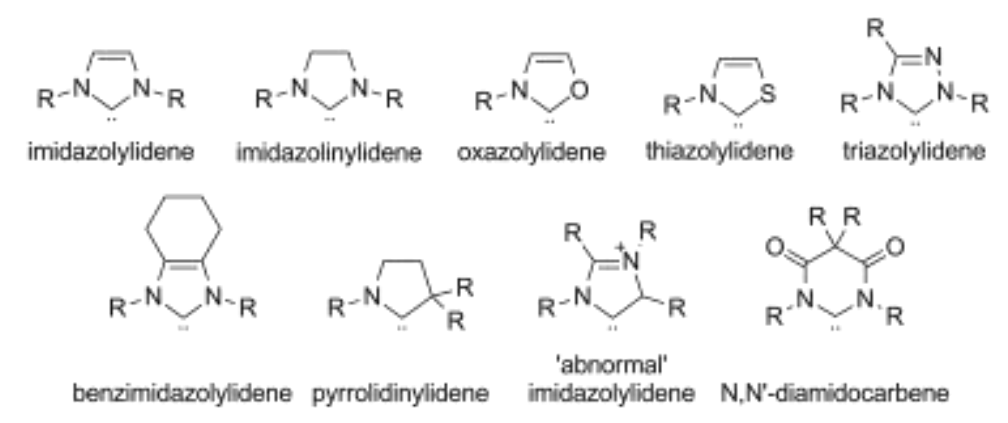

Figure 8: Different classes of familiar NHCs exhibiting structural and electronic differences.

NHCs have been shown to interact with a variety of reaction partners. Via the nucleophilic lone pair, the carbene can form donative bonds to $p$-block elements such as boron, phosphorus or silicon, giving rise to activated small molecules to be used as reagents in chemical synthesis [96-98]. In combination with sterically demanding boranes, frustrated Lewis pairs are formed, which have the potential to activate molecular hydrogen [99]. In addition, NHCs have been found to stabilize highly reactive species like radicals or $p$-block elements in their zero oxidation state by adduct formation [100-103]. Furthermore, NHCs can act as organocatalysts for reactions involving aldehydes, esters and Michael acceptors to facilitate, among others, umpolung [104, 105], transesterification or polymerization [106].

The main focus in NHC studies has, however, been put on their employment as ligands in classic coordination chemistry and consequently on the catalytic activity of the derived metal complexes:

NHC complexes have been reported for all transition metals [107] and furthermore adducts for alkali, alkali earth [108] and f-block elements [109] were observed. Owed to their strong $\sigma$-donating character and an additional $\pi$ backbonding capacity, carbene-metal bonds are relatively short and strong, unless excessive steric bulk arises from the nitrogen substituents [110], which renders the bond between metal atom and NHC ligand highly temperature and oxidatively stable [111]. Consequently, NHCs were found to tightly bind to metal surfaces, where they can be further functionalized [112] or serve to stabilize metal nanoparticles [113]. In metal complexes, the high level of $\sigma$-donation leads to an electron-rich metal center that is highly activated for oxidative addition. Hydrogenation reactions can be 
catalyzed by appropriate Ir- and Ru-based complexes [114] while $\pi$-bond activation is often achieved by employment of corresponding gold complexes [115]. Highest levels of research attention is paid to the catalysis of cross-coupling [116-118] and olefin metathesis reactions $[119,120]$, which are commonly mediated by NHC-complexes containing Pd and Ru, respectively, as the center ion.

\subsection{Objective of this Work}

The objective of this work is separated into two sections. In the first segment, the behavior of different substrates under examination using SABRE is assessed. In previous studies of individual substrate compounds, the focus has often been on optimizing certain aspects of the SABRE procedure, e.g., polarization field strength or temperature, in order to attain optimal results for the particular molecule of interest $[31,47,49,56]$. Here, the attention is turned onto the differences in behavior observed when certain chemical aspects of the substrate molecules are altered. This can be achieved by modification of the molecular structure by, for example, changing the size of a ring system or by changing substitution patterns or substituents of existing substrate molecules. Furthermore, it is of interest to evaluate if such described modifications influence the observed behavior in the same way, when different polarization transfer catalyst systems are employed.

The second objective of this work lies in the synthesis of a solid-phase bound $N$-heterocyclic carbene to be employed as a ligand in SABRE polarization transfer experiments. The purpose of this synthesis lies in the creation of a potent polarization transfer catalyst that can feasibly be separated from the remaining components of the sample solution by means of filtration. By doing so, metal-free substrate solutions can be prepared which consequentially exhibit a greatly reduced toxicity towards live organisms. These solutions can then be injected into said organisms with the dissolved hyperpolarized analyte molecules acting as a powerful contrast agent, thereby paving the way for in situ application of the SABRE technique in MRI. 


\section{Theory}

\subsection{Parahydrogen}

The term parahydrogen is used to describe the nuclear spin isomer $\alpha \beta-\beta \alpha$ of the hydrogen molecule $\mathrm{H}_{2}$, in which the nuclear spins exhibit an antiparallel orientation toward one another. Its existence is owed to the Pauli Exclusion Principle, which requires the overall wavefunction

$$
\Psi=\Psi_{t} \Psi_{v} \Psi_{r} \Psi_{e} \Psi_{n}
$$

of spin- $-1 / 2$ particles (fermions) to be antisymmetric in the exchange of the nuclei. Since the translational contribution $\Psi_{t}$ does not affect the overall symmetry aspect and only the even ground states of $\Psi_{v}$ and $\Psi_{e}$ are populated at room temperature, the product of the rotational and the nuclear spin contribution $\Psi_{r} \Psi_{n}$ is required to be antisymmetric. As a consequence, $\mathrm{H}_{2}$ molecules in the antisymmetric nuclear spin state, i.e., parahydrogen, must inevitably populate even rotational quantum states with $j=0,2,4$ etc., while orthohydrogen molecules in the nuclear triplet states $\alpha \alpha, \beta \beta$ or $\alpha \beta+\beta \alpha$ are necessarily in odd rotational states. This correlation is exploited in the preparation of $p \mathrm{H}_{2}$, which is achieved by cooling hydrogen gas to low temperatures where mainly the rotational ground state is populated, thereby forcing the gas molecules to adopt the nuclear $\alpha \beta-\beta \alpha$ state. The temperature dependent ortho-to-para ratio is given by

$$
\frac{N_{o}(T)}{N_{p}(T)}=\frac{3 \sum_{j=0,2, \ldots}(2 j+1) e^{-j(j+1) \theta_{r} / T}}{\sum_{j=1,3, \ldots}(2 j+1) e^{-j(j+1) \theta_{r} / T}}
$$

with $j$ being the rotational quantum number and $\theta_{r}$ being the rotational temperature $\left(\theta_{r}=\right.$ $\frac{h c B}{k}$, where $B$ is the rotational constant of $\mathrm{H}_{2}$ and $h$ is the Planck constant) [15]. The equilibrium parahydrogen fraction of $\mathrm{H}_{2}$ gas in dependence of the temperature is displayed in Figure 9, which shows the high and low temperature limits of 25 and $100 \%$, respectively.

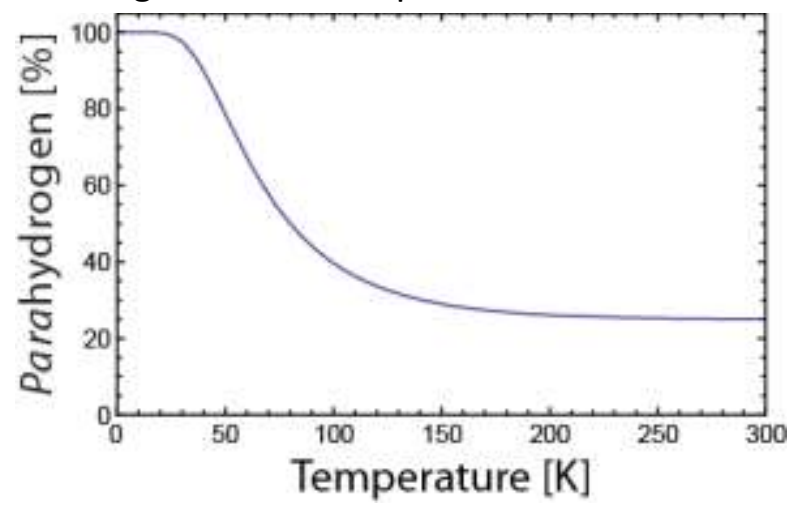

Figure 9: Thermal equilibrium parahydrogen fraction in $\mathrm{H}_{2}$ gas in dependence of the temperature.

As the ortho-to-para transition is spin forbidden, a paramagnetic catalyst is required in order to kinetically facilitate the conversion. In the absence of a suitable catalyst the rate of conversion is low, and the lifetime of non-equilibrium ortho/para mixtures is on the order of days to weeks. 


\subsection{Chemical and Phenomenological Description of SABRE}

The employed precatalyst complexes $\left[\operatorname{Ir}(\mathrm{COD})\left(\mathrm{PC} y_{3}\right)(\mathrm{py})\right]^{+}$and $[\operatorname{Ir}(\mathrm{COD})(\mathrm{NHC}) \mathrm{Cl}](\mathrm{NHC}=\mathrm{IMes}$, SIMes) exhibit a square planar molecular geometry, which is typical for $\operatorname{Ir}(I)$ complexes due to their electronic $d^{8}$ configuration. Upon treatment with min. three equivalents of substrate in methanol and exposure to an excess of parahydrogen at room temperature, the octahedral $\operatorname{Ir}(\mathrm{III})$ complexes $\left[\operatorname{Ir}(\mathrm{H})_{2}\left(\mathrm{PCy}_{3}\right)(\text { substrate })_{2}(\mathrm{py})\right]^{+}$and $\left[\operatorname{Ir}(\mathrm{H})_{2}(\mathrm{NHC})(\text { substrate })_{3}\right](\mathrm{C}$, Scheme 4) are readily formed under hydrogenation of the cyclooctadiene ligand [25, 34].

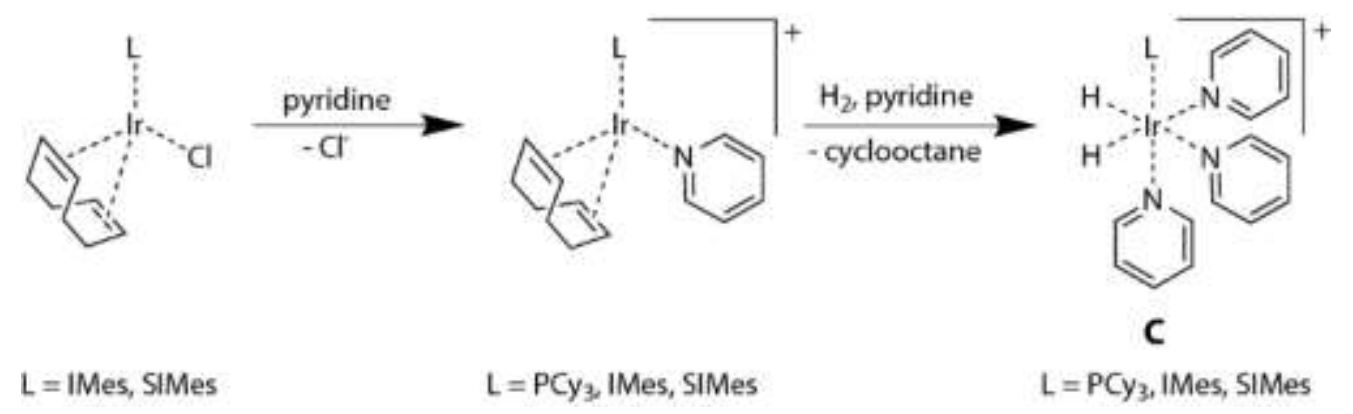

Scheme 4: Preparation of the active polarization transfer catalyst starting from the precatalysts $[\operatorname{Ir}(\mathrm{COD})(\mathrm{NHC}) \mathrm{Cl}](\mathrm{NHC}=\mathrm{IMes}, \mathrm{SIMes})$ and $\left[\operatorname{Ir}(\mathrm{COD})\left(\mathrm{PC} \mathrm{y}_{3}\right)(\mathrm{py})\right]^{+}$. Here, pyridine is used as an exemplary substrate compound.

In these complexes, the hydride ligands and two of the substrate molecules occupy the equatorial binding sites. The third substrate ligand is bound in an axial position and trans to the $\mathrm{PC}_{3}$ or NHC ligand. In ${ }^{1} \mathrm{H}$ NMR spectra, the formation of the dihydride complexes is indicated by the observation of hydride resonances with a strong high field shift in the range between -20 and $-24 \mathrm{ppm}$ (Figure 10). The exact chemical shift values depend on the employed ligand [45] and the analyte bound in trans position to the respective hydride [53].

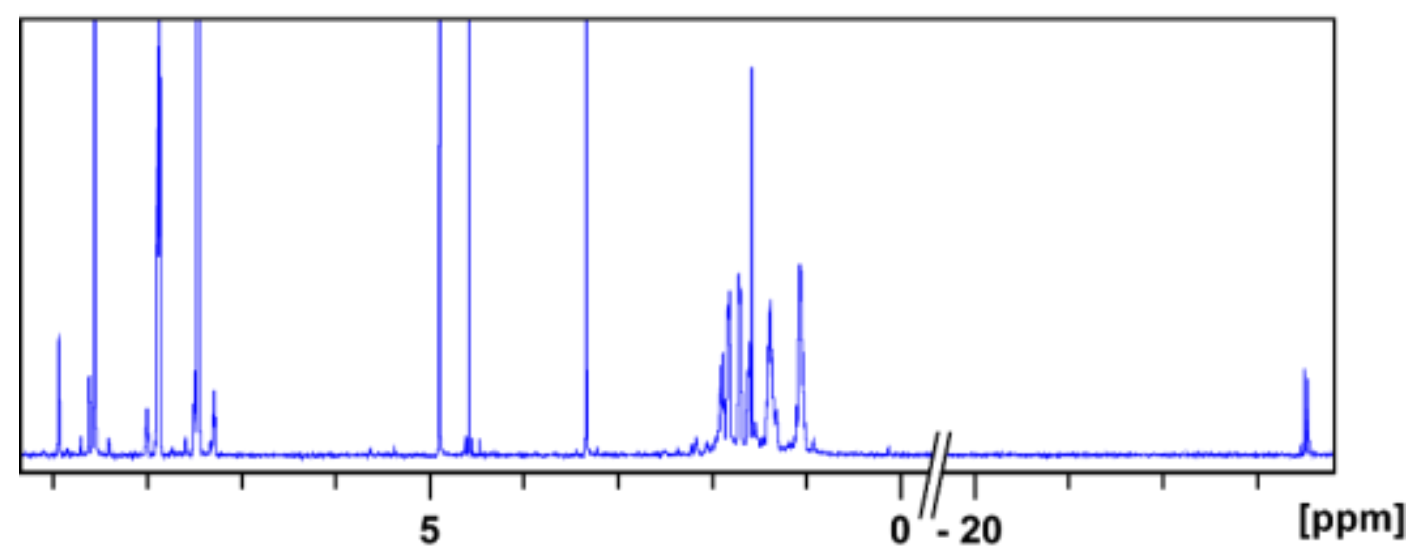

Figure 10: $1 \mathrm{H}$ NMR spectrum of an activated polarization transfer catalyst C (Scheme 4). Resonances between 1 and $2 \mathrm{ppm}$ arise from the employed phosphine ligand tricyclohexyl phosphine while the peaks in the aromatic region originate from the substrate pyridine, either in its free form or as a ligand in the iridium complex. The resonance at $-23.5 \mathrm{ppm}$ stems from the hydride ligands. Remaining signals arise from water, hydrogen gas or the solvent MeOD.

Examination of ligand exchange pathways using DFT calculations and EXSY data revealed a dissociative ligand exchange mechanism for the equatorially bound substrate molecules, while axially bound ligands do not undergo an exchange [25, 34]. At sufficiently high analyte 
concentrations, a vacant equatorial coordination site is quickly repopulated [51]. Similarly, exchange of the hydride ligands was found to also occur via dissociation of a substrate ligand, followed by coordination of a $\mathrm{H}_{2}$ molecule to form the intermediate complex $\left[\operatorname{Ir}(\mathrm{H})_{2}\left(\mathrm{H}_{2}\right)(\mathrm{L})(\text { substrate })_{2}\right]^{+}\left(\mathrm{L}=\mathrm{PCy} \mathrm{y}_{3}, \mathrm{IMes}\right.$, SIMes) $\left(\mathrm{C}^{\ddagger}\right.$, Scheme 5) [31]. As dissociation of the analyte from the complex is the rate-limiting step in the substrate exchange, its rate constant is independent of the analyte concentration [34]. Increased substrate concentrations do, however, cause an adverse effect on the exchange rate of the hydrides, as the $\mathrm{H}_{2}$ molecules compete with substrate molecules for the coordination of the free complex binding site after the substrate dissociation step. An increase in hydrogen pressure increases the $\mathrm{H}_{2}$ concentration and therefore causes the opposite effect, increasing the hydride exchange rate [31]. A thorough description of the reaction kinetics is given in section 2.3 .

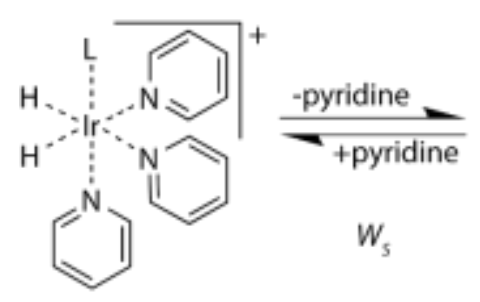

C

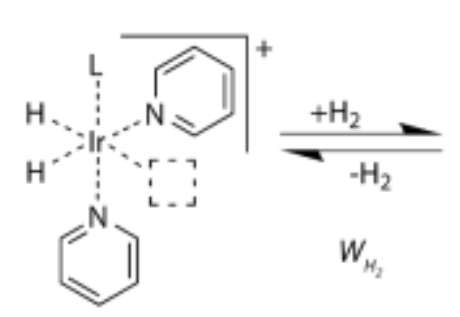

$\mathbf{C}^{+}$

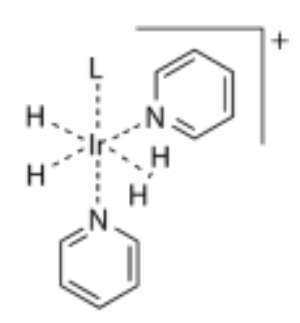

$\mathbf{C}^{\ddagger}$

Scheme 5: Substrate and Hydride exchange pathways in the active SABRE polarization transfer catalyst. Here, pyridine is employed as an exemplary substrate. $\mathrm{L}=\mathrm{PC} \mathrm{y}_{3}$, IMes, SIMes.

In order to form the active polarization transfer catalyst, two equatorial as well as one axial position in the complex must be occupied using suitable substrate molecules, necessitating a substrate-to-Ir ratio of not less than 3:1 [25, 34, 51]. In order to attain signal enhancement of free substrate resonances, an excess of analyte is required, to allow for hyperpolarized molecules to accumulate in solution while the complex remains active. If the excess, however, exceeds the amount of substrate that can reversibly bind to the complex over the experimental run time, the efficiency of hyperpolarization is reduced. Optimal substrate-tocatalyst ratios have been determined to be 5:1 for Crabtree's catalyst [25] and between 9:1 [51] and 10:1 [34] for $[\operatorname{Ir}(\mathrm{COD})(\mathrm{IMes}) \mathrm{Cl}]^{+}$. Increasing the parahydrogen pressure results in an increase of the achievable enhancement as larger amounts of polarization are available in the form of solvated $\mathrm{pH}_{2}$ molecules [29]. Reducing the total number of spins that can receive polarization from the $\mathrm{pH}_{2}$ nuclei, e.g., by deuteration, has a similar effect, as the total amount of available polarization is distributed among fewer nuclei [35, 59]. Lowering the absolute substrate concentration towards sub-mM levels causes a loss of SABRE activity, as vacant coordination sites in the iridium complex are not efficiently re-coordinated by substrate molecules, resulting in the formation of inactive, solvent-coordinating complexes [51].

\subsection{Kinetic Description of SABRE Processes}

This section is based on ref. [81]. 
As the substrate exchange was found to be a dissociative process [25, 34], the substrate exchange rate $W_{S}$ depends solely on the catalyst concentration $[C]$ :

$$
W_{S}=k_{S}^{d}[C]
$$

Here, $k_{S}^{d}$ represents the substrate dissociation rate constant.

On the other hand, the exchange rate of hydrogen $W_{H_{2}}$ depends on the concentration of the short-lived $\left(\mathrm{H}_{2}\right)(\mathrm{H})_{2}$ complex $C^{\ddagger}$ (Scheme 5)

$$
W_{H_{2}}=k_{H_{2}}^{d}\left[C^{\ddagger}\right]
$$

( $k_{\mathrm{H}_{2}}^{d}$ being the dissociation rate constant of molecular hydrogen) for which quasi-stationary conditions apply:

$$
\left[C^{\ddagger}\right]=\frac{k_{H_{2}}^{a}}{2 k_{H_{2}}^{d}}\left[H_{2}\right]\left[C^{\dagger}\right]
$$

Here, $k_{H_{2}}^{a}$ is the association rate constant of molecular hydrogen.

As substrate $S$ and complex $C$ are in chemical exchange, concentration of $C^{\dagger}$ is determined by:

$$
\left[C^{\dagger}\right]=\frac{k_{S}^{d}[C]}{k_{S}^{a}[S]}=K_{S} \frac{[C]}{[S]}
$$

The hydrogen exchange rate via the active catalyst species can therefore be formulated as:

$$
W_{H_{2}}=\frac{k_{H_{2}}^{a}}{2}\left(\frac{k_{S}^{d}[C]}{k_{S}^{a}[S]}\right)\left[H_{2}\right]=\frac{k_{H_{2}}}{[S]}[C]\left[H_{2}\right]=k_{H_{2}}^{\prime}[C]\left[H_{2}\right]
$$

Furthermore, $\left[H_{2}^{*}\right]$ describes the nuclear spin state imbalances of the hydrogen gas molecules, which is given as the difference in numbers between molecules in their singlet and triplet state:

$$
\left[H_{2}^{*}\right]=\left[H_{2}(|S\rangle)\right]-\frac{1}{3} \sum_{i=1,0,-1}\left[H_{2}\left(\left|T_{i}\right\rangle\right)\right]=\left(x_{p}-\frac{x_{o}}{3}\right)\left[H_{2}\right]=\frac{4 x_{p}-1}{3}\left[H_{2}\right]
$$

Here, $x_{p}$ and $x_{o}$ denote the para- and orthohydrogen fractions, respectively. Upon association with the SABRE catalyst complex, the spin state imbalance is transferred to the hydrides in the complex:

$$
\left[C^{*}\right]=\left[C_{|S\rangle}\right]-\frac{1}{3} \sum_{i=1,0,-1}\left[C_{\left|T_{i}\right\rangle}\right]
$$

Similarly, by nuclear spin state conversion, the imbalance is transferred onto the substrate nuclei:

$$
\left[S^{*}\right]=\left[S_{|\beta\rangle}\right]-\left[S_{|\alpha\rangle}\right]=\left(x_{p}+\frac{x_{o}}{3}-\frac{2 x_{o}}{3}\right)[S]=\frac{4 x_{p}-1}{3}[S]
$$


Now, five reactions can be postulated that affect the spin state imbalances $\left[H_{2}^{*}\right],\left[C^{*}\right]$ and [S*] of the species $\mathrm{H}_{2}, \mathrm{C}$ and $S$, which represent the hydrogen gas, the active polarization transfer complex and the substrate molecule, respectively:
a) $\quad \mathrm{C}+\mathrm{H}_{2}^{*} \stackrel{k_{\mathrm{H}_{2}}^{\prime}}{\longrightarrow} \mathrm{C}^{*}+\mathrm{H}_{2}$
b) $\quad C^{*} \stackrel{R_{C}}{\rightarrow} 0$
c) $\quad C^{*} \stackrel{\lambda k_{S}^{d}}{\longrightarrow} C_{1}+S^{*}$
d) $\quad C_{1}+S^{*} \stackrel{\lambda^{\prime} k_{S}^{a}}{\longrightarrow} C^{*}$
e) $\quad S^{*} \stackrel{R_{S}}{\rightarrow} 0$

Here, a) describes the association of the active polarization transfer catalyst $C$ with parahydrogen and c) constitutes the release of hyperpolarized substrate from the resulting complex $C^{*}$. Reaction d) describes the opposite event, i.e., the re-association of $S^{*}$ with the nuclear equilibrium catalyst complex, which poses as an additional relaxation pathway for the hyperpolarized substrate. By inclusion of factors $\lambda$ and $\lambda^{\prime}$, the nuclear polarization transfer efficiency in c) and d) is taken into account. Reaction equations b) and e) indicate time-dependent relaxation of the hyperpolarized species governed by the corresponding relaxation rate constants $R_{C}$ and $R_{S}$.

The time-dependent kinetic equations for $\left[C^{*}\right]$ and $\left[S^{*}\right]$ can therefore be derived to be:

$$
\begin{gathered}
\frac{d\left[C^{*}\right]}{d t}=k_{H_{2}}^{\prime}\left[H_{2}^{*}\right][C]-\left(R_{C}+\lambda k_{S}^{d}\right)\left[C^{*}\right]+\lambda^{\prime} k_{S}^{a}\left[C^{\dagger}\right]\left[S^{*}\right] \\
\frac{d\left[S^{*}\right]}{d t}=\lambda k_{S}^{d}\left[C^{*}\right]-\left(R_{S}+\lambda^{\prime} k_{S}^{a}\left[C^{\dagger}\right]\right)\left[S^{*}\right]
\end{gathered}
$$

Due to the usually employed high $\mathrm{pH}_{2}$ flow rate, $\left[\mathrm{H}_{2}^{*}\right]$ is assumed to immediately reach the steady state condition and is considered as constant.

When, after time $T$ of parahydrogen exposure, the steady state of the system is reached, i.e.,

$$
\frac{d\left[C^{*}\right]}{d t}=0 ; \frac{d\left[S^{*}\right]}{d t}=0
$$

the concentrations of $C^{*}$ and $S^{*}$ amount to

$$
\begin{array}{r}
{\left[C^{*}\right]=k_{H_{2}}^{\prime}\left[H_{2}^{*}\right][C] \frac{R_{S}+\lambda^{\prime} k_{S}^{a}\left[C^{\dagger}\right]}{R_{C} R_{S}+R_{C} \lambda^{\prime} k_{S}^{a}\left[C^{\dagger}\right]+\lambda k_{S}^{d} R_{S}}} \\
=\frac{k_{H_{2}}^{\prime}\left[H_{2}^{*}\right][C]\left(R_{S}+\lambda^{\prime} k_{S}^{d} \frac{[C]}{[S]}\right)}{R_{S}\left(R_{C}+\lambda k_{S}^{d}\right)+R_{C} \lambda^{\prime} k_{S}^{d} \frac{[C]}{[S]}}
\end{array}
$$

and 


$$
\begin{aligned}
{\left[S^{*}\right]=k_{H_{2}}^{\prime}\left[H_{2}^{*}\right][C] \frac{\lambda k_{S}^{d}}{R_{C} R_{S}+R_{C} \lambda^{\prime} k_{S}^{a}\left[C^{\dagger}\right]+\lambda k_{S}^{d} R_{S}} } \\
=k_{H_{2}}^{\prime}\left[H_{2}^{*}\right][C] \frac{\lambda k_{S}^{d}}{R_{S}\left(R_{C}+\lambda k_{S}^{d}\right)+R_{C} \lambda^{\prime} k_{S}^{d} \frac{[C]}{[S]}}
\end{aligned}
$$

Finally, using the expression

$$
k_{H_{2}}^{\prime}=\frac{k_{H_{2}}}{[S]}
$$

the enhancement $|\varepsilon|$, which can be described as the ratio of hyperpolarized substrate $S^{*}$ to the total amount of substrate $S$, can be given as:

$$
|\varepsilon|=\eta|P|=\eta \frac{\left[S^{*}\right]}{[S]}=\eta \frac{4 x_{p}-1}{3} \frac{\left[H_{2}\right][C]}{[S]^{2}} \frac{\lambda k_{S}^{d} k_{H_{2}}}{R_{S}\left(R_{C}+\lambda k_{S}^{d}\right)+R_{C} \lambda^{\prime} k_{S}^{d} \frac{[C]}{[S]}}
$$

Herein, $\eta$ denotes the theoretical maximum enhancement factor of 50\% (see Section 2.5).

\subsection{Strong Coupling and Level Anti-Crossings}

When given states of two distinguishable systems depend on the modulation of a parameter in different ways, the trajectories of these states can intersect at certain values without influencing each other (Level Crossing, LC) (Figure 11a).
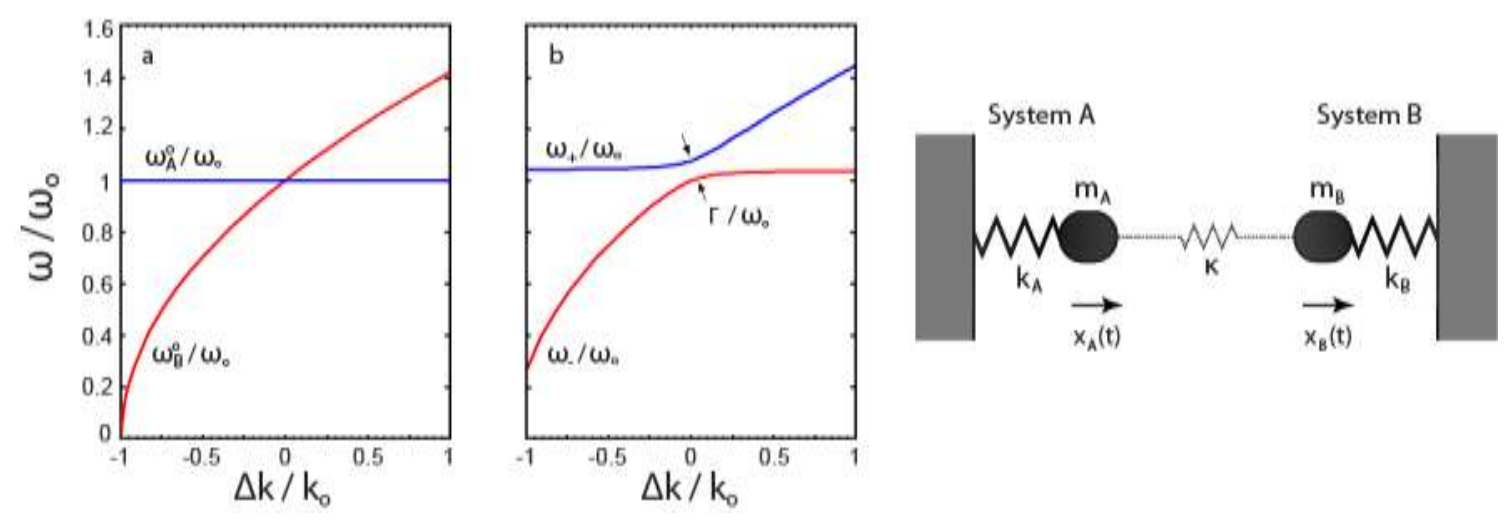

Figure 11: Oscillation frequencies of systems $A$ and $B$ depending on the spring constant of system $B$ with spring constants $k_{A}=k_{0}$ and $k_{B}=k_{0}+\Delta k$. In (a), no coupling is present between the oscillators and the oscillation frequencies are independent of each other. In (b), a coupling of $\kappa=0.08 k_{0}$ was introduced between the oscillators. Under these conditions, a crossing of the oscillation frequencies is avoided and their trajectories exhibit a characteristic frequency splitting of $\Gamma=\frac{\sqrt{\kappa / m_{A}} \sqrt{\kappa / m_{B}}}{\sqrt{\omega_{A} \omega_{B}}}$. This figure was adapted from [121].

If a coupling between the two systems is introduced, the systems are no longer independent of each other and a change made to one of them will also have an effect on the other, the extent of which is determined by the relative size of the coupling. For coupling constants that are small compared to the observed quantity, the systems can for the most part be considered individually with only minor perturbations imposed by the coupling. If, however, the magnitude of the coupling is comparable to the difference in the affected quantity, the two systems cannot be regarded detached from one another and have to be considered as a 
combined system. This is commonly referred to as strong coupling. In this case, the trajectories of the modulated states can no longer cross when approaching each other, but instead repel each other and interchange their trajectories beyond the intended point of crossing (Figure 11b) [121]. This is referred to as a Level Anti-Crossing (LAC).

\subsubsection{Strong Coupling in NMR}

When considering atomic nuclei, the energy levels of individual nuclear quantum states depend on the magnetic field experienced by the nuclear spins (Zeeman Effect). This general dependence is given by

$$
E=-\gamma m \hbar B_{0}
$$

where $\gamma=$ gyromagnetic ratio of the considered nucleus, $m=$ magnetic quantum number, $\hbar=$ reduced Planck constant and $B_{0}=$ external magnetic field. For a nucleus with a nuclear spin of $I=1 / 2, m$ can take the values $+1 / 2$ and $-1 / 2$ and the transition energy between these two nuclear states is given by:

$$
\Delta E=\left|E_{-1 / 2}-E_{+1 / 2}\right|=\gamma \hbar B_{0}
$$

This energy difference can be converted into the corresponding photon frequency $v$ using

$$
v=\frac{\Delta E}{h}
$$

Inside an NMR magnet with a field strength of $B_{0}=141 \mathrm{kGs}$, these frequencies are on the order of $600 \mathrm{MHz}$, which is equivalent to the precession frequency of the rotating reference frame. Various effects, e.g., electron density, lead to a shielding of the atomic nucleus from the external magnetic field, which changes the effective magnetic field $B_{\text {eff }}$ experienced by the individual nuclei. This correlation is given by

$$
B_{\text {eff }}=(1-\sigma) B_{0}
$$

with $\sigma$ being the shielding constant. Substituting equations 18 and 20 into equation 19 highlights the modulation of the nuclear precession frequency by this shielding effect:

$$
v=\frac{\gamma(1-\sigma) B_{0}}{2 \pi}
$$

The difference in the precession frequencies of two nuclei $\Delta v=v_{2}-v_{1}$ is therefore given by

$$
\Delta v=\frac{\gamma\left(1-\sigma_{2}\right) B_{0}}{2 \pi}-\frac{\gamma\left(1-\sigma_{1}\right) B_{0}}{2 \pi}=\left(\sigma_{1}-\sigma_{2}\right) \frac{\gamma B_{0}}{2 \pi}
$$

and for homonuclei inside the NMR magnet usually on the order of several $\mathrm{kHz}$ over the applicable frequency range. The presence of $B_{0}$ on the right-hand side of equation 22, however, indicates the dependence of $\Delta v$ on the magnitude of the prevalent magnetic field, which allows for the modulation of precession frequency differences by adjustment of the magnetic field strength. In contrast to the precession frequency, the size of the J-coupling, which is the effect a nuclear spin state of a given nucleus exerts on the energy levels of another nucleus, is independent of the prevalent magnetic field. 
In NMR spectroscopy, the strong coupling condition is met when the difference between the nuclear transition energies of two coupled nuclear spins $I$ and $J$, which can be expressed as the nuclear precession frequency difference $\Delta v_{I, J}$, is comparable to the scalar coupling $J_{I, J}$ between them, $\Delta v_{I, J} \sim J_{I, J}$. Chemically equivalent spins have identical nuclear transition energies $(\Delta v=0)$ and are therefore, if a scalar coupling is present $(J \neq 0)$, strongly coupled independent of the experienced magnetic field. In order to observe strong coupling in an unequal proton pair inside the high field of the NMR magnet, the chemical shift difference must be minimal, as ${ }^{1} \mathrm{H}-{ }^{1} \mathrm{H} J$-couplings rarely exceed values of $15 \mathrm{~Hz}$ while the observed frequencies span a significantly wider range. Due to the field dependence of the Zeeman splitting, however, strong coupling of such pairs with even a considerable chemical shift difference can be readily achieved by transferring the spins to lower magnetic fields. This can be illustrated by solving equation 22 for $B_{0}$,

$$
B_{0}=\frac{2 \pi}{\gamma} \frac{\Delta v}{\left(\sigma_{2}-\sigma_{1}\right)}
$$

and inserting the applicable values for a system of interest.

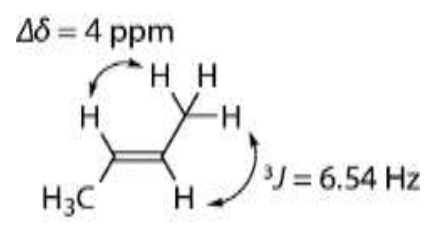

Figure 12: Chemical structure and NMR data of trans-2-butene.

In trans-2-butene for instance (Figure 12), two proton resonances can be observed in the corresponding 1D NMR spectrum, originating from the vinylic and the methyl protons. The vicinal ${ }^{1} \mathrm{H}-{ }^{1} \mathrm{H}$ coupling between these two types of nuclei is ${ }^{3} \mathrm{~J}_{\mathrm{H}, \mathrm{H}}=6.54 \mathrm{~Hz}$, while the chemical shift difference is $\Delta \delta=\left(\sigma_{2}-\sigma_{1}\right)=4 \mathrm{ppm}$. Inside a $600 \mathrm{MHz}$ spectrometer, this corresponds to a precession frequency difference of $2400 \mathrm{~Hz}$, which clearly exceeds the coupling constant by more than two orders of magnitude. From equation 23 with $\frac{\gamma_{H}}{2 \pi}=42.576 \mathrm{MHzT}^{-1}$ and $\Delta v={ }^{3} J_{\mathrm{H}, \mathrm{H}}=6.54 \mathrm{~Hz}$, the magnetic field at which the precession frequency is identical to the scalar coupling constant can be calculated to be $B_{0}=384 \mathrm{Gs}$, in which case the strong coupling condition is met.

For heteronuclear spin pairs, however, the difference in precession frequencies is on the order of $\mathrm{MHz}$ and consequently several orders of magnitude higher than the corresponding scalar couplings. In this case, strong coupling is inaccessible inside the NMR magnet and considerable magnetic shielding of the NMR sample is necessary in order to minimize the magnetic field and to fulfill the strong coupling requirements. Magnetic field values on the order of $<10 \mathrm{mGs}$ were determined to achieve the strong coupling effect for a ${ }^{1} \mathrm{H}_{-}{ }^{15} \mathrm{~N}$ spin pair, which is significantly below the earth's magnetic field of $\sim 0.5 \mathrm{Gs}$ [60].

\subsubsection{Nuclear Level Anti-Crossings in SABRE model complexes}

Theoretical descriptions of the SABRE polarization transfer mechanism have been presented in a number of recent publications [37, 66, 78, 80-82]. This section is based thereupon. 
The requirements for the occurrence of level anti-crossings between nuclear spin states are an energy matching of these states and a scalar coupling of the corresponding nuclei [78]. For a system of two spin $1 / 2$ nuclei, due to the Zeeman splitting, the energy levels of the $\alpha \beta$ and the $\beta \alpha$ states always approach each other at magnetic fields $B_{0} \rightarrow 0$. Owed to their scalar coupling $J$, however, the levels do not intersect, but remain split by a value of $V=J$. For larger spin systems, additional LACs are observed at non-zero fields, which have to be determined by quantum mechanical considerations.

For the quantum mechanical description, the Hamiltonian of the spin system is separated into two components:

$$
\widehat{H}=\widehat{H}_{0}(B)+\widehat{H}_{1}
$$

Here, the zero-order element $\widehat{H}_{0}(B)$ depends on the magnetic field strength and describes the energy levels $E_{\mathcal{K}}$ and $E_{\mathcal{L}}$ of eigenstates $|\mathcal{K}\rangle$ and $|\mathcal{L}\rangle$, so that level crossings (LC) of spin states can be determined for a field $B_{L C} . \widehat{H}_{1}$, however, describes a perturbation of the system, e.g., by an additional scalar coupling, due to which the eigenstates of the system are no longer adequately described by $|\mathcal{K}\rangle$ and $|\mathcal{L}\rangle$ alone, but by mixed states of the two. This results in an interconversion of the corresponding populations and an avoided crossing that exhibits a splitting of

$$
V=2\left\langle\mathcal{K}\left|\widehat{H}_{1}\left(B_{L C}\right)\right| \mathcal{L}\right\rangle
$$

between the two states at the location of the intended level crossing. For the two-spin system mentioned above, this results in the separation value $V=J$ at $B_{L A C}=0$.

In the theoretical descriptions mentioned above, model complexes based on the equatorial plane of the octahedral polarization transfer catalyst (C, Scheme 5) were used in order to derive the underlying theoretical aspects of SABRE. These model systems contain the nuclear spin pair $A$ and $A^{\prime}$, which represents the hydride ligands in chemically equivalent positions of the mirror-symmetric iridium complex. The hydrides exhibit a scalar coupling between them $\left(J_{A, A^{\prime}} \neq 0\right)$ and are consequently strongly coupled independent of the magnitude of the prevalent magnetic field, so that it is permissible to described them with either the singlet states $S$ or one of the triplet states $T_{+}, T_{0}$ or $T_{\text {. }}$ of a two-spin system. Depending on the complexity of the examined model, selected further spins representing substrate or ligand nuclei were incorporated.

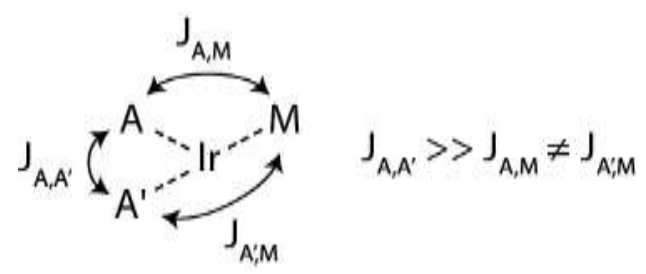

Figure 13: Three-spin model complex for the discussion of the SABRE polarization transfer mechanism based on the equatorial arrangement of the active polarization transfer catalyst. $A$ and $A^{\prime}$ denote the hydride spins originating from a parahydrogen molecule whereas $M$ represents a spin in the substrate molecule. The iridium nucleus is NMR inactive and serves as a connecting piece. 


\subsubsection{AA'M three-spin system}

The simplest model system for SABRE consists of the hydride spins $A$ and $A^{\prime}$, as well as spin $M$, which corresponds to a single spin $1 / 2$ nucleus in the substrate molecule (Figure 13 ). In this model, the chemical shifts of $A$ and $A^{\prime}$ are identical and different from that of the substrate $\left(\delta_{A}=\delta_{A^{\prime}} \neq \delta_{M}\right)$. Furthermore, coupling $J_{\mathrm{A}, \mathrm{A}^{\prime}}$ is considerably larger than couplings $J_{\mathrm{A}, \mathrm{M}}$ and $J_{A^{\prime}, M}$ to the substrate nucleus, which are non-identical $\left(J_{A, M} \neq J_{A^{\prime}, M}\right)$. At sufficiently low magnetic fields, the spin system is transferred into the strong coupling regime, so that eight mixed spin terms of the type $|S, K\rangle$ and $\left|T_{k}, L\right\rangle$ are formed. Therein, $S$ and $T_{k}$ represent the four singlet and triplet states mentioned above and $K$ and $L$ are either the $\alpha$ or the $\beta$ state of the substrate nucleus. When it is assumed that $\left(\delta_{A}-\delta_{M}\right)<0$ and $J_{A, A^{\prime}}<0$, the energies of spin states $|S \alpha\rangle$ and $\left|T_{+} \beta\right\rangle$ are, disregarding couplings $J_{\mathrm{A}, \mathrm{M}}$ and $J_{\mathrm{A}^{\prime}, \mathrm{M}}$, given as:

$$
E_{S \alpha}=-\frac{v_{M}}{2}-\frac{3 J_{A, A \prime}}{4} \text { and } E_{T_{+} \beta}=-v_{A}+\frac{v_{M}}{2}+\frac{J_{A, A}}{4}
$$

These energies are identical when the condition

$$
v_{A}-v_{M}=J_{A, A}
$$

is met, thereby defining the location of a level crossing. The perturbation of the system by $\widehat{H}_{1}$ is given by the additional couplings $J_{\mathrm{A}, \mathrm{M}}$ and $J_{\mathrm{A}^{\prime}, \mathrm{M}}$, which cause a level splitting by

$$
V=\frac{\left(J_{A, M}-J_{A^{\prime}, M}\right)}{\sqrt{2}}
$$

and which transforms the LC into an LAC. From equation 28, it can be seen that the asymmetry of the scalar couplings from the hydrides to the substrate nucleus $\left(J_{A, M} \neq J_{A^{\prime}, M}\right)$ is crucial for the splitting of the states and it is this magnetic inequivalence of the chemically equivalent hydride spins that perturbs the Hamiltonian of the spin system and allows for the mixing of the two considered spin states at the level anti-crossing.

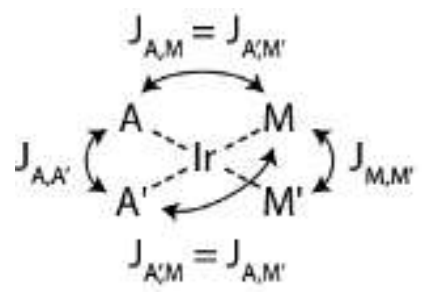

Figure 14: Four-spin model complex consisting of the hydride spin pair $A A^{\prime}$ and the substrate spin pair $M M^{\prime}$. In this model, $J_{A, A^{\prime}}$ is considerably larger than the remaining scalar couplings and the individual hydride spins exhibit inequivalent couplings to the two substrate spins $\left(J_{A, M} \neq J_{A, M^{\prime}}\right)$. Furthermore, $J_{A, M}=J_{A^{\prime}, M^{\prime}}$ and $J_{A^{\prime}, M}=J_{A, M^{\prime}}$. The iridium nucleus is NMR inactive and therefore disregarded.

\subsubsection{AA'MM' four-spin system}

By inclusion of an additional substrate spin $\mathrm{M}^{\prime}$, the model is transformed into a four-spin system (Figure 14), in which spins $\mathrm{M}$ and $\mathrm{M}^{\prime}$ are also chemically equivalent $\left(\delta_{A}=\delta_{A^{\prime}} \neq\right.$ $\delta_{M}=\delta_{M}$ ). Consequently, the substrate spins in this model can also be described using the singlet-triplet-basis $\left(\mathrm{S}_{,} \mathrm{T}_{+}, \mathrm{T}_{0}\right.$ or $\left.\mathrm{T}_{-}\right)$and mixed terms of the type $|K L\rangle$ are formed between the two strongly coupled spin pairs in the low field regime, in which $K$ and $L$ describe the spin states of the $A A^{\prime}$ and the $\mathrm{MM}^{\prime}$ pair, respectively. Furthermore, the model complex is 
assumed to be symmetric in that $J_{A, M}=J_{A^{\prime}, M^{\prime}}$ and $J_{A, M^{\prime}}=J_{A^{\prime}, M}$, yet $J_{A, M} \neq J_{A^{\prime}, M}$ still holds true. The Zeeman splitting of the four nuclei and the scalar couplings within the $A A^{\prime}$ and $M M^{\prime}$ pairs are included in the zero-order Hamiltonian $\widehat{H}_{0}$, while the four couplings between hydride and substrate spins are combined into $\widehat{H}_{1}$ as perturbations. For spin states involving the singlet state of the hydride spins, the following level crossing conditions can be determined:

$$
\begin{array}{cc}
\left|S T_{ \pm}\right\rangle \leftrightarrow\left|T_{ \pm} S\right\rangle: & \pm\left(v_{A}-v_{M}\right)=J_{A, A^{\prime}}-J_{M, M \prime} \\
|S S\rangle \leftrightarrow\left|T_{ \pm} T_{ \pm}\right\rangle: & \pm\left(v_{A}-v_{M}\right)=J_{A, A^{\prime}}+J_{M, M^{\prime}}-\frac{1}{2}\left(J_{A M}+J_{A M \prime}\right)
\end{array}
$$

Due to spin level mixing by $\widehat{H}_{1}$, these level crossings are turned into level anti-crossings. The locations of the LACs are mainly determined by the difference in the chemical shifts of the hydride and substrate spins as well as the size of the hydride-hydride coupling $J_{A, A^{\prime}}$. The additional $J$-couplings in equations 29 and 30 are usually significantly smaller than $J_{A, A^{\prime}}$ and are therefore considered secondary contributions.

Both the three-spin and the four-spin system are suitable to describe active SABRE polarization transfer catalyst systems which incorporate NHC ligands and in which no axially bound ligand nucleus is coupled to the hydride and substrate spins. Calculated field dependences for both model systems are given in Figure 15 together with the applied NMR data. The results for both models show a strong similarity, as only a single extremum is present, which is owed to the relative proximity of the two LACs derived for the $A A^{\prime} M M^{\prime}$ model. It must, however, be noted that this proximity is owed to the NMR parameters used in the calculations, which were derived from NMR data of active polarization transfer complexes using pyridine as the analyte. If, by modification of the active complex, the contributions of the secondary scalar coupling become more pronounced, a separation of the LAC locations is possible. However, the results obtained in these calculations are in good agreement with experimental field dependence data recorded using NHC-containing SABRE catalysts $[34,45]$.
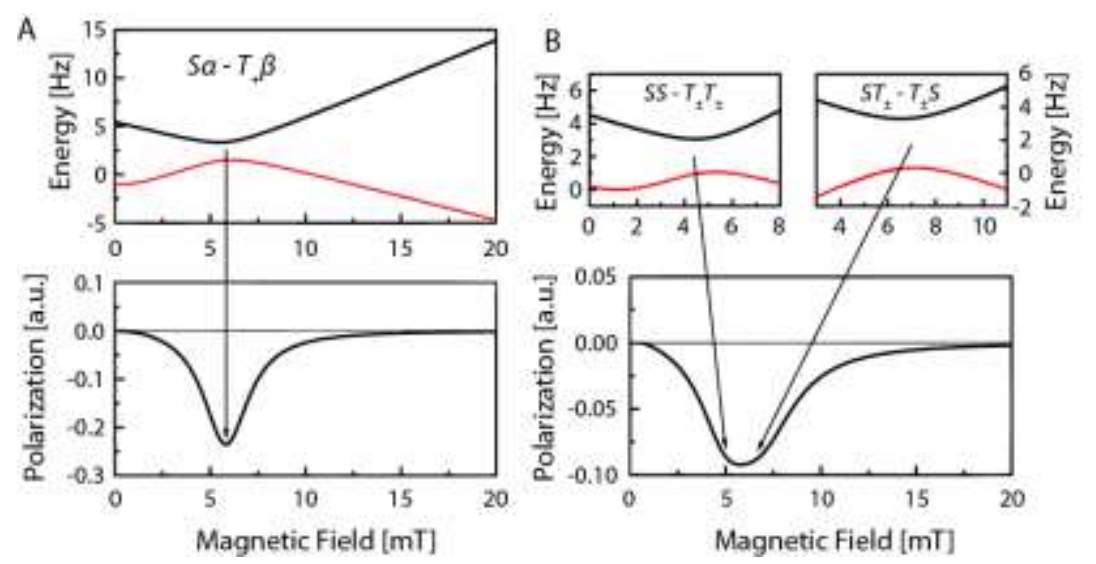

$$
\begin{aligned}
& \delta_{M}-\delta_{M}=31 \mathrm{ppm} \\
& J_{M N}=-7 \mathrm{~Hz} \\
& J_{M M}=J_{N N}=3 \mathrm{~Hz} \\
& J_{X M}=J_{M M}=0 \mathrm{~Hz} \\
& J_{X M}=0 \mathrm{~Hz} \\
& J_{M N}=2 \mathrm{~Hz}
\end{aligned}
$$

Figure 15: Calculated field dependences of SABRE attained for the three- and four-spin model systems $A A^{\prime} M$ and $A A^{\prime} M M^{\prime}$ using the $N M R$ parameters given on the right-hand side. $(A)$ depicts the results for the $A A^{\prime} M$ three-spin system, for which only a single LAC was determined, and which gives rise to a single emissive maximum. The results for the $A A^{\prime} M M^{\prime}$ four-spin system are given in (B). Here, two locations of LACs were 
identified, which, however are not separated and form a single emissive maximum. This figure was reproduced from the supplementary data of ref. [37].
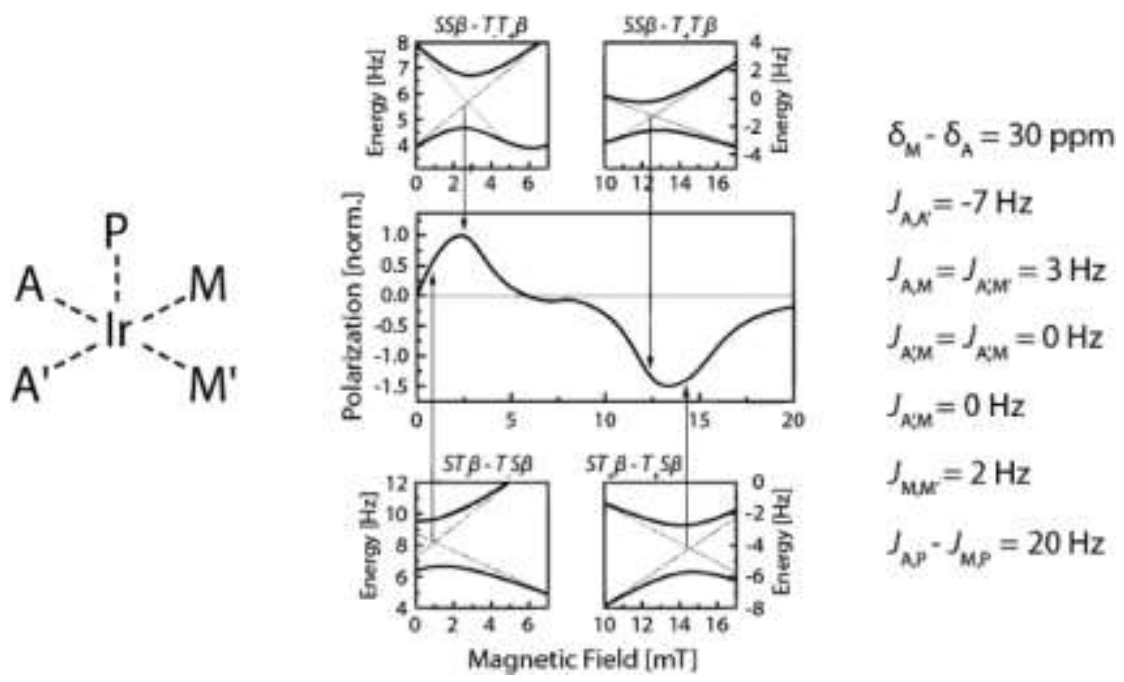

Figure 16: Structure of the five-spin model complex $A A^{\prime} M M^{\prime} P$ and calculated field dependence of SABRE obtained using the NMR parameters on the right-hand side. As a result of coupling of the ${ }^{31} \mathrm{P}$ nucleus in the phosphine ligand to the hydride spins, the number of LACs is doubled compared to the $A A^{\prime} M^{\prime} M^{\prime}$ four-spin model. Due to their relative proximity, pairs of two LACs overlap and give a combined maximum, resulting in only one absorptive and one emissive maximum from four level anti-crossings. This figure was reproduced from ref. [37].

\subsubsection{AA'MM'P five-spin system}

In phosphine containing polarization transfer catalysts like Crabtree's catalyst, additional heteronuclear couplings are present due to the ${ }^{31} \mathrm{P}$ spin in axial position, which was added into this version of the model complex as spin $\mathrm{P}$ (Figure 16). For this system, it was assumed that $J_{A, P}=J_{A^{\prime}, P} \gg J_{M, P}=J_{M^{\prime}, P}$, while the remaining interactions within the $A A^{\prime} M M^{\prime}$ system keep their validity. Similar to the previous model, mixed terms of the kind $|K L M\rangle$ are formed, in which $K$ and $L$ once more refer to the singlet and triplet states of the pairs $\mathrm{AA}^{\prime}$ and $\mathrm{MM}^{\prime}$, while $M$ denotes either the $\alpha$ or the $\beta$ state of the phosphorus spin. Due to the large precession frequency difference between the phosphorus and the proton spins, the ${ }^{31} \mathrm{p}$ nucleus is not strongly coupled to the remaining nuclei at low field, yet owed to the significantly sized scalar coupling, the precession frequencies of the $A A^{\prime}$ and the $\mathrm{MM}^{\prime}$ spin pairs are modulated according to

$$
v_{A} \rightarrow v_{A}-J_{A, P} I_{Z}^{P} \text { and } v_{M} \rightarrow v_{M}-J_{M, P} I_{Z}^{P}
$$

where $I_{z}^{P}= \pm \frac{1}{2}$ is the $z$-projection of the ${ }^{31} \mathrm{P}$ nucleus and $J_{A, P}$ and $J_{M, P}$ are the scalar coupling constants between the phosphorus spin and the hydride or substrate spins, respectively. As a result of the signal splitting, the total number of level anti-crossings is doubled and their conditions for states including the hydride singlet term are given as:

$$
\left|S T_{ \pm} \beta\right\rangle \leftrightarrow\left|T_{ \pm} S \beta\right\rangle: \quad \pm\left(v_{A}-v_{M}\right)=J_{A, A^{\prime}}-J_{M, M^{\prime}} \pm\left(J_{A, P}-J_{A M}\right) I_{Z}^{P}
$$




$$
\begin{aligned}
|S S \beta\rangle \leftrightarrow\left|T_{ \pm} T_{ \pm} \beta\right\rangle: \quad \quad \pm\left(v_{A}-v_{M}\right)= & J_{A, A^{\prime}}+J_{M, M^{\prime}}-\frac{1}{2}\left(J_{A M}+J_{A M \prime}\right) \\
& \pm\left(J_{A, P}-J_{A M}\right) I_{Z}^{P}
\end{aligned}
$$

Consequently, four LACs of interest for SABRE can be determined of which, once more, pairs of two overlap in corresponding field dependence calculations as a result of the only minor contributions of $J_{M, M^{\prime}}, J_{A, M}$ and $J_{A, M^{\prime}}$, as depicted in Figure 16 . These calculated results are, especially regarding the bimodal dependence pattern, in very good agreement with experimentally obtained results using Crabtree's catalyst [35].

\subsection{Mechanism of the Polarization Transfer}

For simplicity reasons, the discussion of the underlying SABRE mechanism, which is based on the theoretical derivation in the supplementary information of ref. [81], are described for the $A A^{\prime} M$ three-spin system.

For an assumed $p \mathrm{H}_{2}$ fraction of 1 , all newly formed polarization transfer complexes in solution are in the singlet state in respect to the hydride spins $A$ and $A^{\prime}$. The substrate spin $\mathrm{M}$, however, is only thermally polarized and in almost equal parts in the $\alpha$ and $\beta$ state, so that the three-spin model complex exhibits the mixed spin states $|S \alpha\rangle$ and $|S \beta\rangle$. At the determined $L A C$, the $|S \beta\rangle$ state remains unaffected because its energy level remains isolated. The $|S \alpha\rangle$ state, however, interchanges with the $\left|T_{+} \beta\right\rangle$ state, leading to a depopulation of the substrate $\alpha$ state and consequentially to an enrichment of the substrate $\beta$ state. The polarization $P$ of the substrate spin $M$ can be given as:

$$
P=\frac{M_{\alpha}-M_{\beta}}{M_{\text {total }}}
$$

As described above, the location of a level crossing can be derived using the zero-order Hamiltonian $\widehat{H}_{0}$, which describes the energy levels of the spin system. The splitting between the energy levels that occurs in the event of a level anti-crossings is caused by the perturbation

$$
\widehat{H}_{1}=\frac{\left(J_{A, B}-J_{A^{\prime}, B}\right)}{2}+J_{A, B}\left[\left(I_{A x}, I_{B x}\right)+\left(I_{A y}, I_{B y}\right)\right]+J_{A^{\prime}, B}\left[\left(I_{A \prime x}, I_{B x}\right)+\left(I_{A \prime y}, I_{B y}\right)\right]
$$

which only contributes in the case of crossing energy levels. The size of the splitting is determined by application to the approaching states

$$
\left\langle T_{+} \beta\left|\widehat{H}_{1}\right| S \alpha\right\rangle=-\frac{\left(J_{A, B}-J_{A^{\prime}, B}\right)}{2 \sqrt{2}}
$$

and results in the following energy difference:

$$
v_{L A C}=\frac{\omega_{L A C}}{2 \pi}=2 \frac{\left(J_{A, B}-J_{A^{\prime}, B}\right)}{2 \sqrt{2}}=\frac{\left(J_{A, B}-J_{A^{\prime}, B}\right)}{\sqrt{2}}
$$

The frequency $v_{L A C}$ describes the rate at which the system oscillates between the two spin states during the lifetime of the active polarization transfer catalyst (Figure 17). Upon dissociation of the substrate, the oscillation process stops, and the substrate spin remains in 
its nuclear state when relaxation is disregarded. As the dissociation reaction is distributed in time, the overall substrate polarization averages to a value of $P=-50 \%$, around which the polarization oscillates:

$$
P=\left(\cos 2 \pi v_{L A C} t-1\right) \cdot 50 \%
$$

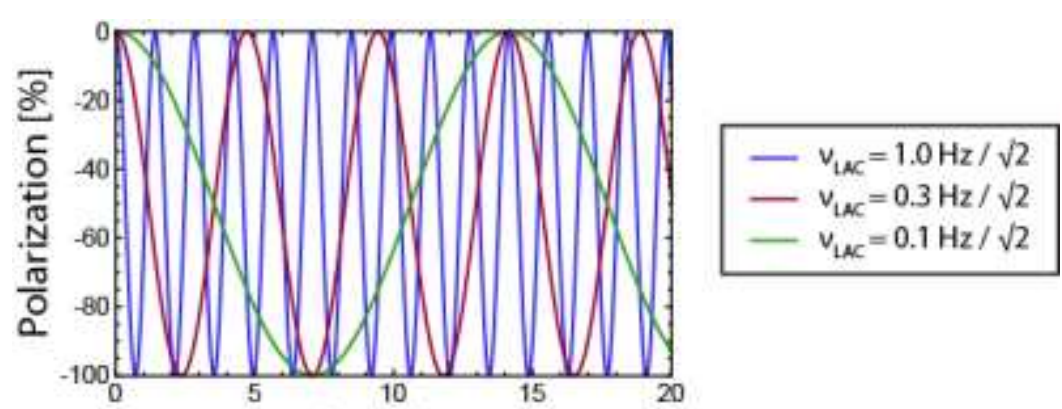

\section{Complex Lifetime [s]}

Figure 17: Polarization of the substrate spin $M$ as a result of the interconversion between $|S \alpha\rangle$ and $\left|T_{+} \beta\right\rangle$ as a function of the complex lifetime. For a larger scalar coupling difference $\left(J_{A, B}-J_{A^{\prime}, B}\right)$, the rate of oscillation is increased.

As $v_{L A C}$ depends on the size of the coupling $J_{A, B}$ (equation 37), the interconversion is more rapid within complexes, in which the substrate exhibits a larger coupling to the hydrides. Likewise, for complexes exhibiting a low $\Delta J=\left(J_{A, B}-J_{A^{\prime}, B}\right)$, achieving maximum enhancement is not feasible when the average complex lifetime is shorter than half an oscillation period.

In-depth theoretical analyses of SABRE relaxation mechanisms as well as exploitation of LACS for high-field SABRE and further theoretical background can be found in refs. $[37,66,78,80$ 82]. 


\section{Results and Discussion}

\subsection{SABRE Hyperpolarization Experiments}

The experimental results obtained using the SABRE hyperpolarization method are summarized in this section.

\subsubsection{Experimental Setup 1}

The following substrates were examined using the shaking table setup and Crabtree's catalyst as the precatalyst:

\subsubsection{Pyrazole and its Derivatives}

Initial experiments using the shaking table setup served to verify the successful setup relocation using previously published results $[35,122]$. As the Boltzmann polarization depends linearly on the magnetic field strength, it is of twice the size for a $600 \mathrm{MHz}$ spectrometer compared to the previously utilized $300 \mathrm{MHz}$ spectrometer. The amount of hyperpolarization transferred from the $\mathrm{pH}_{2}$ nuclei is, however, independent from the magnetic field inside the magnet, so that a decrease in enhancement by a factor of two was expected upon transition to the upgraded experimental setup.

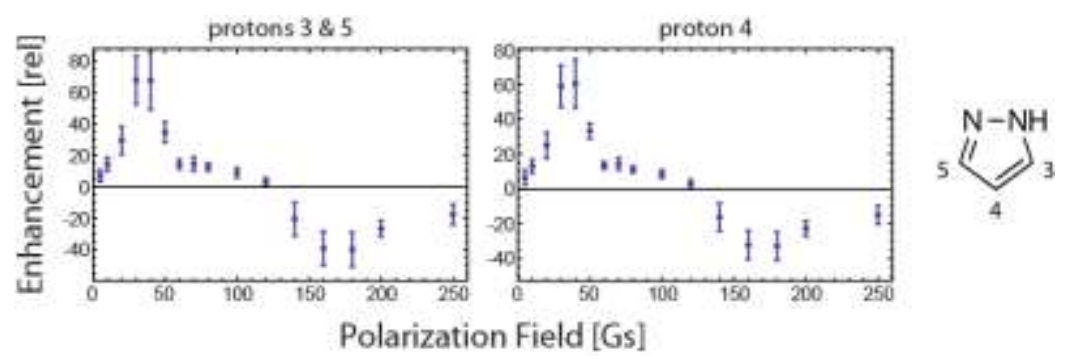

Figure 18: Polarization field dependence of signal enhancement for the protons in pyrazole without pyridine- $d_{5}$ as a secondary substrate. The molecular structure of the substrate molecule is given on the right.

The results obtained for pyrazole with the improved setup (Figure 18) adequately supported this presumption and replicated the previously determined field dependence patterns of the signal enhancement with maximum signal intensities in the regions around 40 and $150 \mathrm{Gs}$ (compare $[35,122])$.

Previously published findings [35] showed an increase in relative signal enhancement when the substrate pyridine was diluted using pyridine- $d_{5}$ as a secondary substrate. Py- $d_{5}$ also coordinates to the active polarization transfer complex but remains NMR silent due to the absence of observable protons. To test the transferability to other substrates, a 9:1 mixture of pyridine- $d_{5}$ as the silent co-substrate and pyrazole as the substrate of interest was examined. As the results (Figure 19) showed a substantial increase in signal enhancement compared to non-diluted pyrazole (Figure 18), this sample composition was selected as default for further investigations using this setup. 


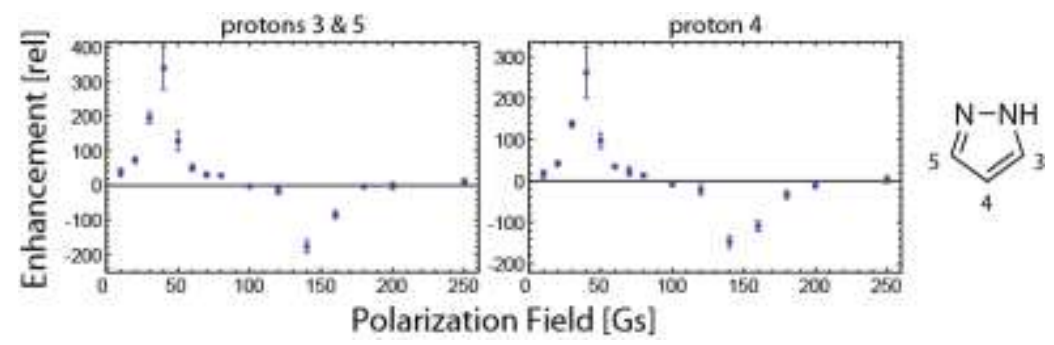

Figure 19: Polarization field dependence of signal enhancement for the protons in pyrazole with pyridine- $d_{5}$ as a secondary substrate. The molecular structure of the substrate molecule is given on the right.

\section{3,5-Dimethylpyrazole}

When examined using the preceding setup, 3,5-dimethylpyrazole had exhibited little [35] to no [122] interaction with the active catalyst. Using the upgraded setup, the combination of improved signal resolution on account of the superior spectrometer and the employment of the dilution method, the substrate showed unambiguous hyperpolarization for all protons in the pattern expected for pyrazole derivatives (Figure 20). The relatively low signal enhancement is attributed to the steric demand of the methyl substituents in positions 3 and 5, which limits the ability of the molecule to approach a binding site with its donor atoms. This results in a weaker binding and a higher exchange rate [40], which can limit the efficiency of the polarization transfer [81]. This is further illustrated by the failed attempts to hyperpolarize the more sterically demanding substrates 3,5-diphenylpyrazole and 3-(1,3benzodioxol-5-yl)-5-(3-bromophenyl)-1H-pyrazole, which exhibit even bulkier substituents in these positions that prevent coordination to the catalytic complex.
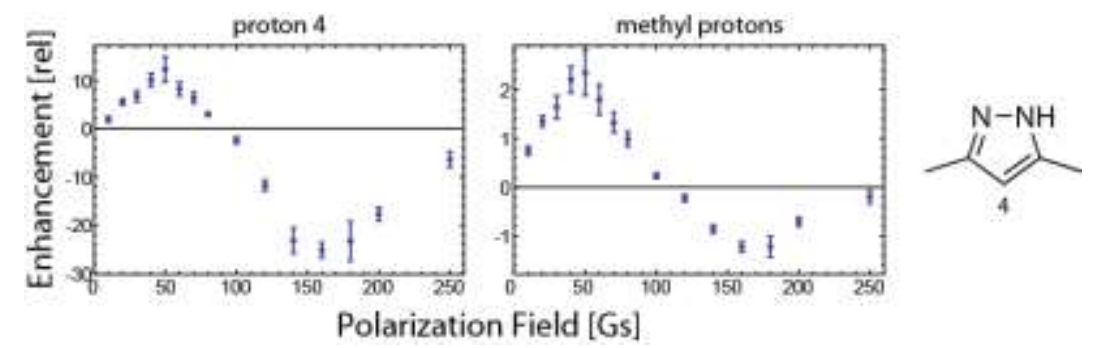

Figure 20: Polarization field dependence of signal enhancement for the protons in 3,5-dimethylpyrazole. The molecular structure of the substrate molecule is given on the right.

\section{4-Methylpyrazole}

The experimental results for 4-methylpyrazole (Figure 21) once more display the expected enhancement pattern for a pyrazole derivative, showing largest signal gain at approx. 40 and $150 \mathrm{Gs}$ and a zero-transition around the $100 \mathrm{Gs}$ mark. The spectra furthermore offer the opportunity to highlight the signal enhancement of the resonances stemming from substrate molecules coordinated to the polarization transfer catalyst, which behaves in the same way as for the free substrates. 

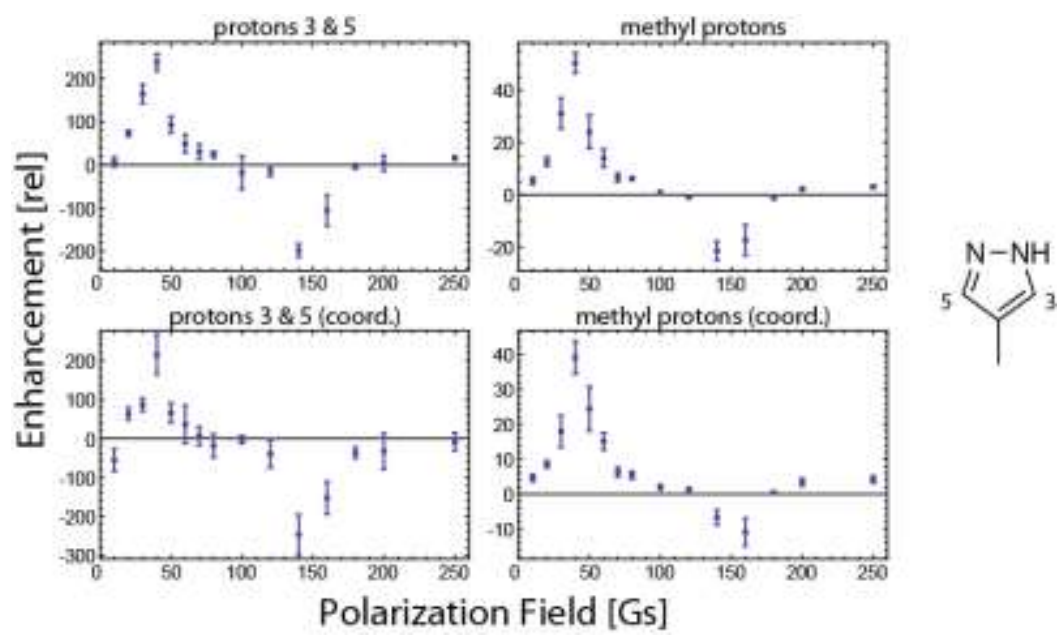

Figure 21: Polarization field dependence of signal enhancement for the protons in 4-methylpyrazole in the free state and when coordinated to the polarization transfer catalyst. The molecular structure of the substrate molecule is given on the right.

\section{3-Aminopyrazole}

Another substrate behaving in an identical fashion is 3-aminopyrazole (Figure 22), which exhibits a relatively strong enhancement for the proton in position 5. Strikingly, it shows no deviation from the established pattern despite the additional nitrogen atom in the amino group. This could possibly act as a further donor atom with a deviating binding motif and corresponding scalar couplings in the active complex, the size of which dictates the efficiency of the polarization transfer [81].

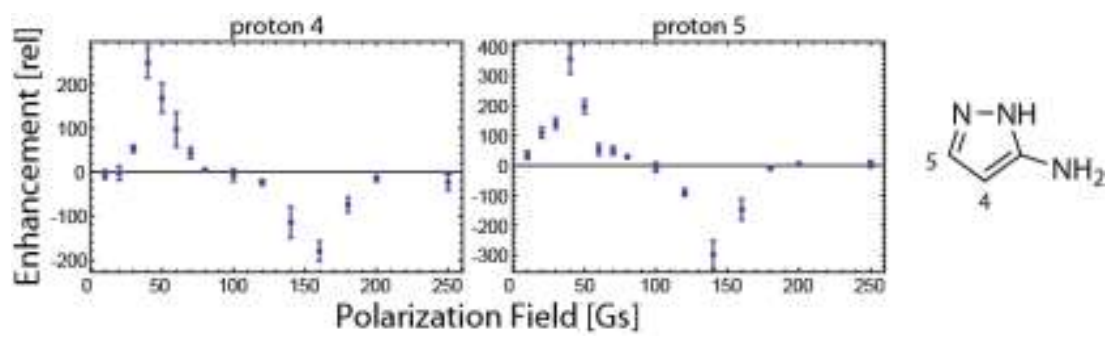

Figure 22: Polarization field dependence of signal enhancement for the protons in positions 4 and 5 of 3-aminopyrazole. The molecular structure of the substrate molecule is given on the right.

\section{3-(4-Aminophenyl)pyrazole}

The same is true for 3-(4-aminophenyl)pyrazole, which features no anomaly regarding the hyperpolarization of its pyrazole protons (Figure 23). The phenyl protons showed a strong overlap with residual pyridine resonances and could not be individually examined due to severe line shape distortions. These originated from fluctuations in the magnetic field caused by the passing of the sample through the magnetic field in the spectrometer. While a polarization of the phenyl protons is probable and implied in the spectra, its extent could not be reliably determined. The described distortion of the line shape in coincidence with significant line broadening posed a permanent impediment to the evaluation of the recorded spectra and constituted a main reason for the decommissioning of this setup. 


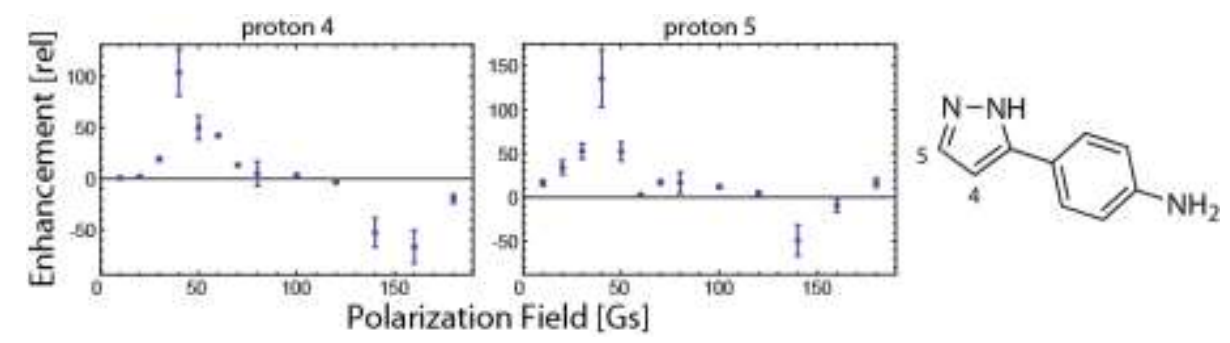

Figure 23: Polarization field dependence for the pyrazole protons in 3-(4-aminophenyl)pyrazole. The molecular structure of the substrate molecule is given on the right.

\section{3,4-Dimethylpyrazole}

The level of hyperpolarization transferred to the protons of 3,4-dimethylpyrazole was unexpectedly low (Figure 24) when compared to other pyrazole derivatives, as no substituent in position 5 obstructed the efficient coordination of the nitrogen nucleus to the catalyst iridium ion. An increased electron density in the pyrazole ring, mediated by the two methyl substituents, may have increased the molecules binding affinity, which in turn reduced the efficient exchange rate at the catalyst, thereby reducing the number of polarized molecules. The enhancement profile, however, once more behaves as expected for a pyrazole derivative.

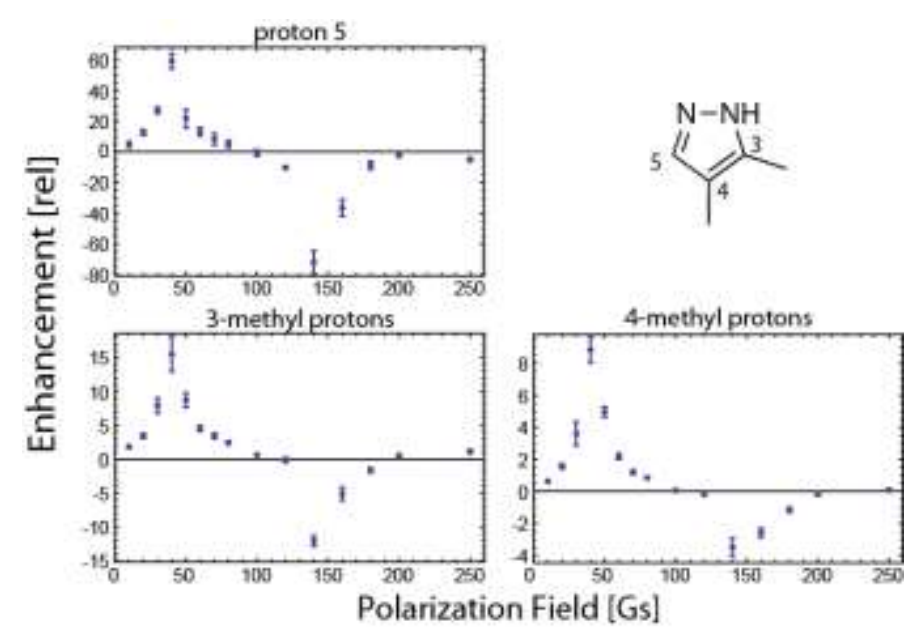

Figure 24: Polarization field dependence of signal enhancement for the protons in 3,4-dimethylpyrazole. The molecular structure of the substrate molecule is given at the top right.

\section{4-Ethoxy-3-methylpyrazole}

In contrast to the previous case, 4-ethoxy-3-methylpyrazole exhibits an ethoxy group in position 4 , which reduces the electron density in the pyrazole ring and thereby counteracts the electron-releasing effect of the methyl group in position 3. The magnitude of the signal enhancement achieved for the ring proton in position 5 as well as the methyl protons in position 3 indicates this to be a beneficial combination (Figure 25). This measurement series further illustrates an interesting phenomenon, as the pattern of hyperpolarization transferred to the protons in the ethoxy group is inverted compared to the anticipated behavior. While the maximum enhancement for these protons is also achieved at magnetic fields of 40 and $150 \mathrm{Gs}$, the sign for the signal intensities changes from negative to positive 
with an increasing polarization field strength. The enhancement pattern for proton 5 and the methyl protons, however, remains unchanged.

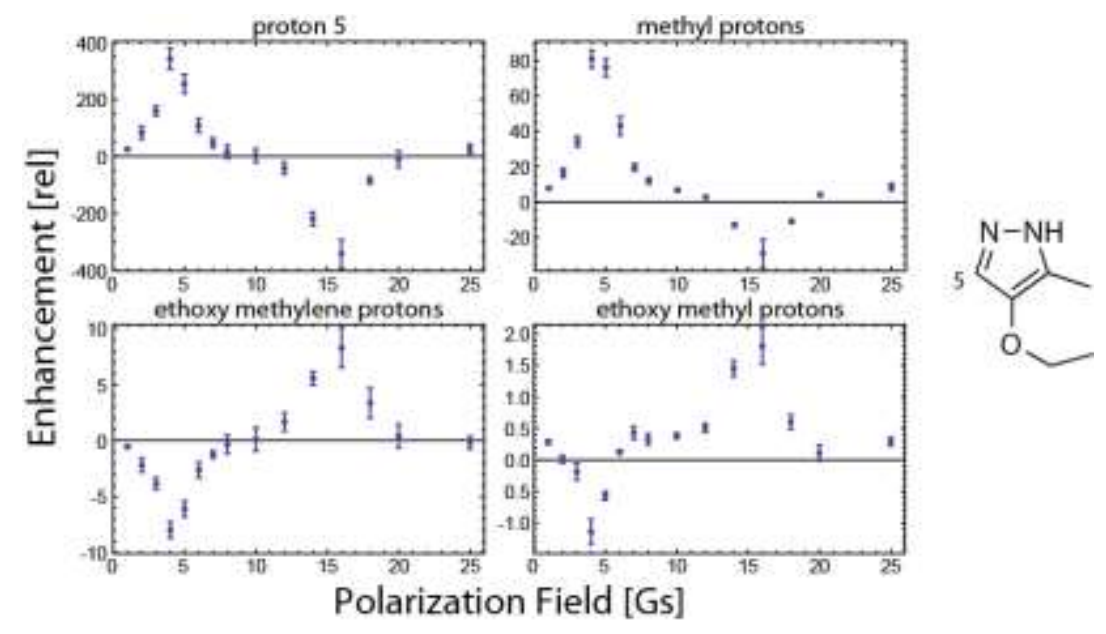

Figure 25: Polarization field dependence of signal enhancement for the protons in 4-ethoxy-3-methylpyrazole. The molecular structure of the substrate molecule is given on the right.

\section{Indazole}

Examination of indazole, which is an annular molecule composed from a pyrazole and a benzene ring structure, once more revealed the expected pyrazole derivative enhancement pattern for all its protons (Figure 26). It further visualizes the feasibility of hyperpolarizing protons in ring structures that do not carry the donor atom themselves but are merely connected to it via an extended scalar coupling network. The amount of polarization detected on these protons is, however, considerably smaller than for the protons in the actual heterocycle.
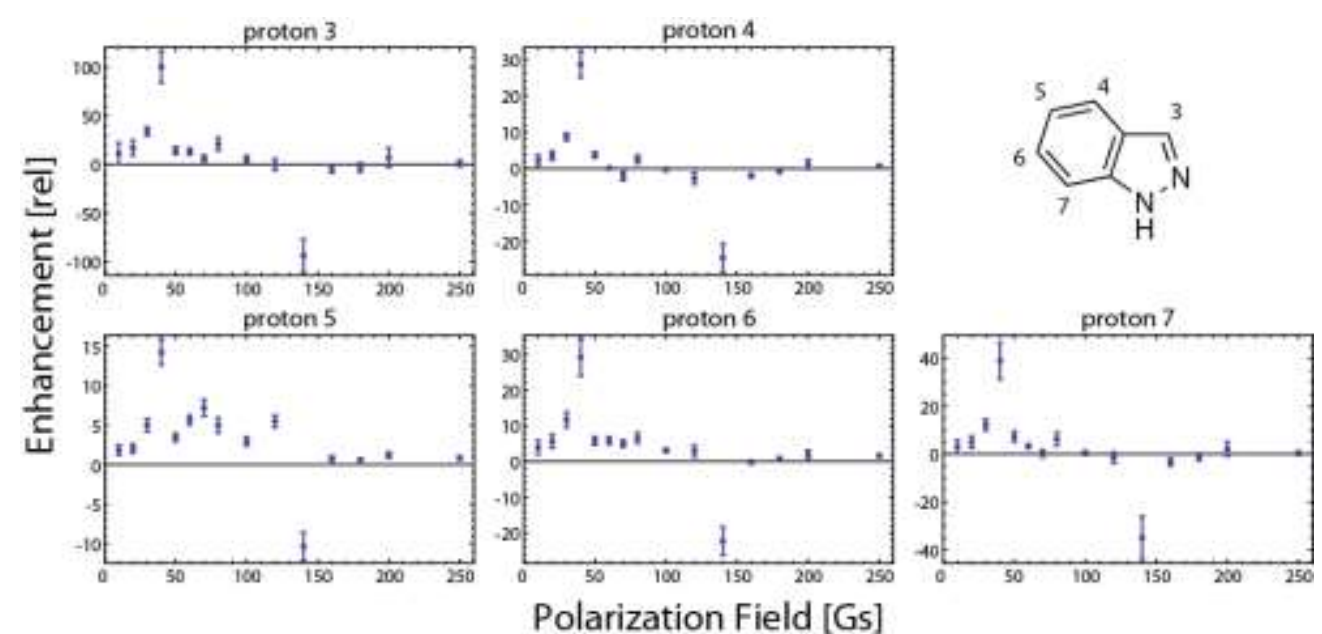

Figure 26: Polarization field dependence of signal enhancement for the protons in indazole. The molecular structure of the substrate molecule is given at the top right.

\section{1,2,3- and 1,2,4-triazole}

1,2,3-Triazole and 1,2,4-triazole are not strictly derivatives of pyrazole, yet show a strong structural resemblance. The determined field dependence curves depict strong similarities between both substrates (Figure 27), as the attained patterns for both molecules seem 
unstructured in the same way. The sign of the proton resonances changes multiple times for both substrates and the enhancement plots appear to be highly disordered.

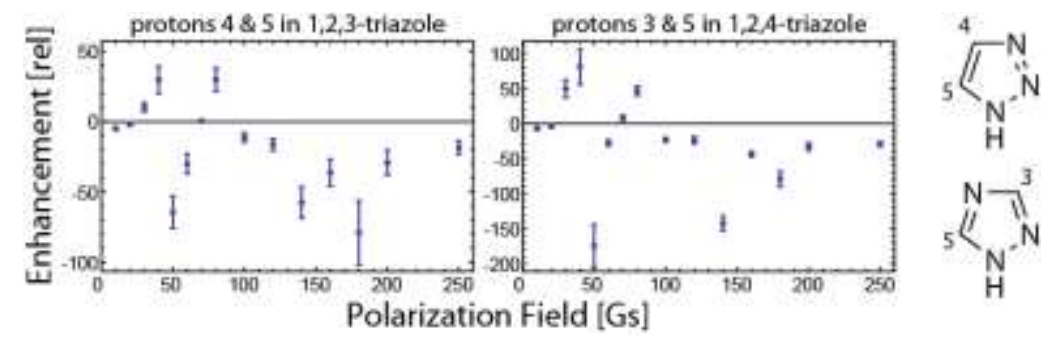

Figure 27: Polarization field dependence of signal enhancement for the protons in 1,2,3-triazole and 1,2,4triazole. The molecular structures of the substrate molecules are given on the right.

Three nitrogen atoms are present in both compounds, giving rise to two different coordination motifs for each structure, which is depicted for 1,2,4-triazole in Figure 28. Pravdivtsev et al. postulated that in the polarization step, one proton species is directly hyperpolarized via its scalar coupling to the parahydrogen nuclei, while the remaining protons obtain their polarization via a redistribution effect within the scalar coupling network of the substrate [80]. It is therefore probable that for motif 1 (Figure 28), in which the protons are identical, polarization is transferred to both protons in an equal fashion. In motifs $2 a$ and $2 b$, however, the two protons are distinguishable while the substrate is coordinated to the complex and only the proton next to the donor atom is directly polarized, as it exhibits a stronger coupling to the hydride ligands. Polarization is then transferred from the first to the second proton via the aforementioned scalar coupling redistribution. The experimental results for 4-ethoxy-3-methylpyrazole (vide supra), in which a change in sign occurred for a polarization transfer via a heteroatom in the substrate as well as previously published data on pyridine $[35,122]$ imply a difference in the resulting enhancement patterns of $\mathrm{H}_{\mathrm{a}}$ and $\mathrm{H}_{\mathrm{b}}$. As the two protons become indistinguishable again upon dissociation of the complex, these patterns as well as the one originating from binding motif 1 are superimposed, presumably resulting in the determined enhancement patterns of 1,2,3- and 1,2,4-triazole.

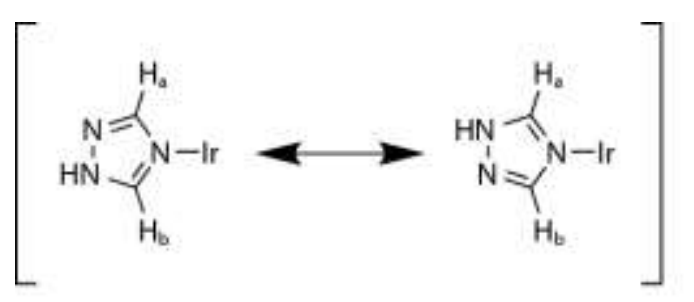

Motif 1

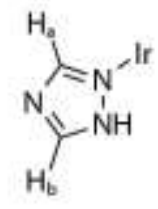

Motif 2a

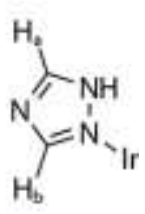

Motif $2 b$

Figure 28: Binding motifs of 1,2,4-triazole to the iridium center in the active polarization transfer complex. Further ligands in the iridium complex are omitted. 


\subsubsection{Pyridine Derivatives}

In a previous work on this polarization technique [122], the three proton resonances of pyridine each showed a very distinct pattern in their field dependence curves. While the ortho resonance almost exclusively experienced a negative signal enhancement over the range of applied polarization field strengths with a well-defined maximum at $130 \mathrm{Gs}$, the opposite was true for the meta resonance, which mainly obtained positive enhancement with a very broad maximum for fields of below $100 \mathrm{Gs}$. The para resonance showed a resemblance to the ortho signal, but also received a notable gain in positive signal intensity at lower polarization fields of around $40 \mathrm{Gs}$.

Computational efforts by Pravdivtsev et al. [80] later supported these findings in a theoretical analysis. In their work, the authors state that the initial transfer of hyperpolarization only occurs to the ortho protons of the bound pyridine, which were calculated to have a scalar coupling to the hydride ligands of the active complex in the range of 1-3 Hz. Polarization of the remaining protons subsequently occurs via a redistribution effect caused by the scalar couplings within the pyridine spin system. It was therefore expected that a change in the scalar coupling system of a substrate by replacement of one of the protons should result in a deviation from the previously determined enhancement patterns.

\section{Nicotinamide}

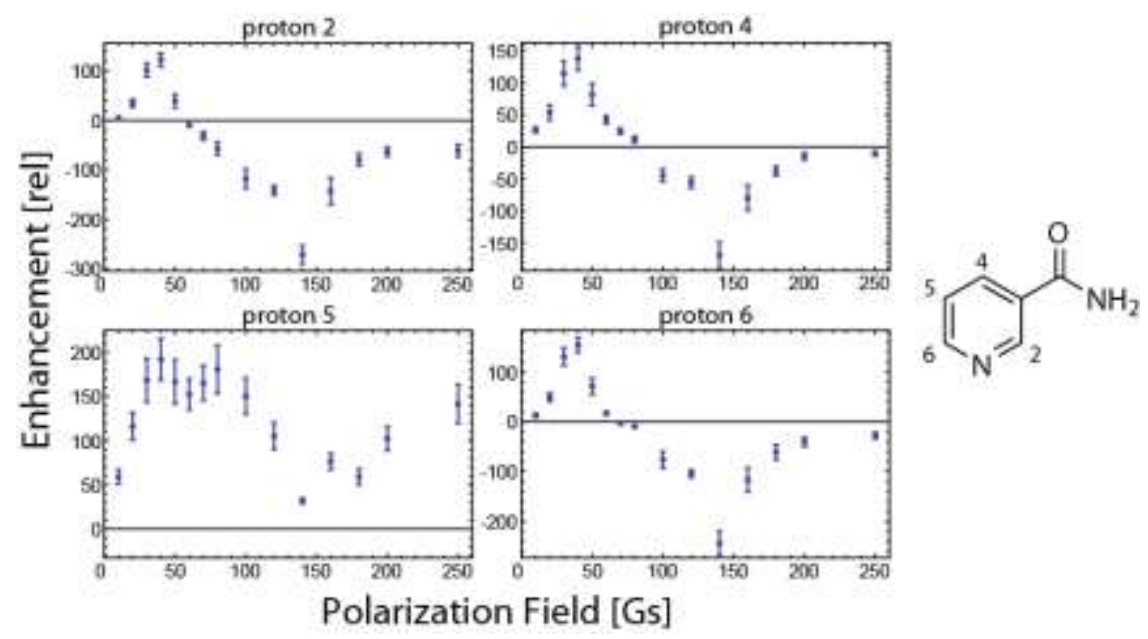

Figure 29: Polarization field dependence of signal enhancement for the protons in nicotinamide. The molecular structure of the substrate molecule is given on the right.

Examination of nicotinamide (pyridine-3-carboxamide) replicated the enhancement pattern observed in pyridine for proton 5 , which is the meta proton to the coordinating nitrogen atom. Over the complete polarization field range, the corresponding signal maintains a positive intensity and exhibits a very broad range of maximum enhancement, which spans the field from 30 to 80 Gs (Figure 29). Protons 2 and 6, which are ortho protons, gain their highest signal intensities at higher fields of around $140 \mathrm{Gs}$ with a negative sign, as is the case for the respective protons in pyridine. For lower fields, however, the protons gain positive signal enhancement of significant size, showing a deviation from unsubstituted pyridine. This 
also extents to proton 4, the para proton in respect to the coordinating nitrogen, which exhibits positive and negative enhancement of nearly the same magnitude at its maxima at 40 and $140 \mathrm{Gs}$, respectively.

\section{3,4,5-Trideuteropyridine}

A rigorous decimation of the spin system by replacement of the meta and para protons using deuterons lead to a further deviation from the enhancement pattern observed for pyridine. The results for 3,4,5-trideuteropyridine (Figure 30) show an extremely efficient polarization transfer to $\mathrm{H} 2$ and $\mathrm{H} 6$, well exceeding a thousandfold gain in signal intensity. This is mostly attributed to the fact that the predominant part of the polarization remains on the ortho protons and is not redistributed to other nuclei. A transfer to the incorporated deuterons, which were not examined, is unlikely, as the strong coupling condition between ${ }^{1} \mathrm{H}$ and ${ }^{2} \mathrm{H}$ nuclei was not fulfilled at the reduced magnetic fields. Furthermore, the hyperpolarization exhibits a very symmetrical pattern, as the absolute value of the enhancement is of the same order of magnitude for the positive maximum at around $40 \mathrm{Gs}$ as well as for the negative maximum at $140 \mathrm{Gs}$.

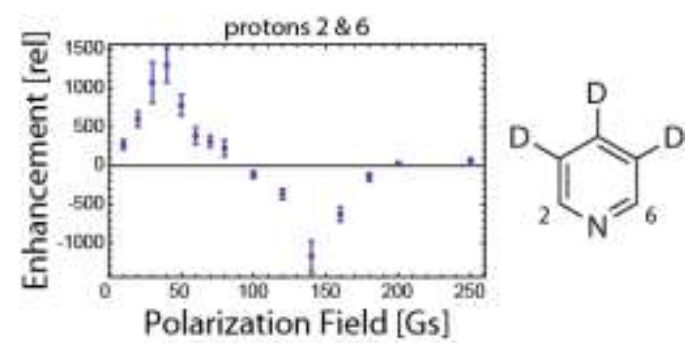

Figure 30: Polarization field dependence of signal enhancement for protons in 3,4,5-trideuteropyridine. The molecular structure of the substrate molecule is given on the right.

\section{2,6-Dideuteropyridine}

As a complementary experiment, 2,6-dideuteropyridine was examined, which shows the inverted substitution pattern compared to the previously examined substrate. In this molecule, no ortho protons are available for direct hyperpolarization and subsequent distribution of polarization to the remaining protons. Therefore, it was of interest to test if an efficient polarization transfer was possible under these circumstances.

The experimental results showed a satisfactory polarization transfer to both the meta and the para protons (Figure 31) albeit of a distinctly smaller magnitude. The meta resonance in this case displays a notably narrower maximum at $40 \mathrm{Gs}$ and also changes to a negative intensity at higher polarization fields. Additionally, the para resonance experiences an unexpected sign flip between 50 and 60 Gs. 


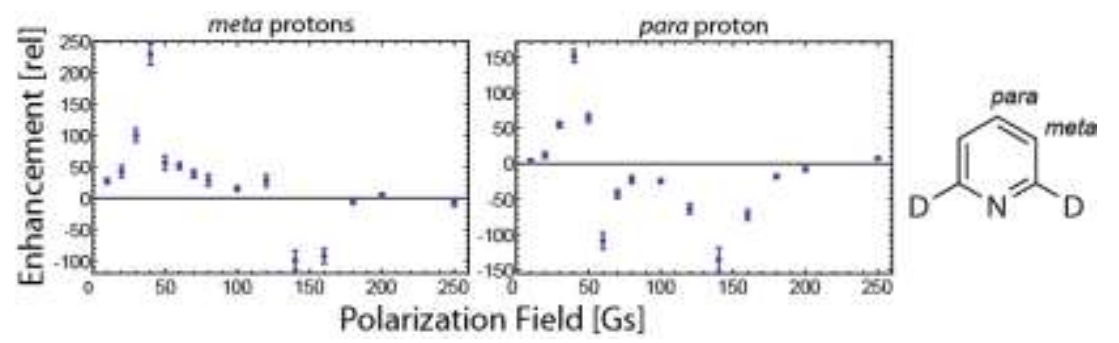

Figure 31: Polarization field dependence of signal enhancement for the meta and para protons of 2,6-dideuteropyridine. The molecular structure of the substrate molecule is given on the right.

It must, however, be noted that owed to the employment of pyridine- $d_{5}$ as a co-substrate, a substantial signal was observed at the chemical shift of the pyridine ortho resonance due to incomplete deuteration. In a later examination without dilution by pyridine- $d_{5}$ and using the Bruker Para-Hydrogen Polariser (See Section 3.1.2.1), the abundance of pyridine ortho signals in the initial reference spectrum was below the detection level. In the first hyperpolarization experiment, however, the enhanced ortho proton resonance was readily observable even at this concentration. In the final reference spectrum, the ortho protons were detectable even without hyperpolarization, indicating a catalyst-mediated H/D exchange increasing the abundance of protons in the pyridine ortho site. Similar behavior has been reported for both $\mathrm{PCy}_{3}$ and $\mathrm{NHC}$ containing catalysts [123, 124]. It can therefore not be excluded that a substantial amount of polarization observed on the meta and para protons stems from initial polarization of ortho protons, as the suggested polarization transfer mechanism indicates [80].

Pyridazine

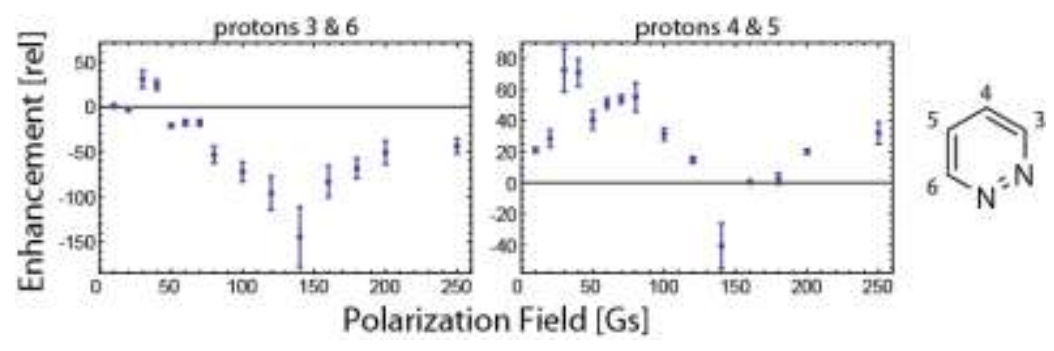

Figure 32: Polarization field dependence of signal enhancement for the protons in pyridazine. The molecular structure of the substrate molecule is given on the right.

Average levels of hyperpolarization were achieved for the protons in pyridazine (Figure 32), a molecule which is similar to pyridine, but possesses two adjacent nitrogen atoms in the ring structure. For this substrate, protons 3 and 6 , which are closest to the coordination site, show behavior similar to ortho protons in pyridine. Protons $4 \& 5$, which are opposite the nitrogen atoms exhibit an indistinct maximum at lower polarization field, indicating a behavior similar to meta protons in pyridine.

\subsubsection{Discussion of Experimental Setup 1}

With the implementation of the shaking table at the $600 \mathrm{MHz}$ Avance III spectrometer, the hyperpolarization setup used in previous publications was successfully transferred to this department and preceding result were reproduced. The improvements to the setup included 
the utilization of a superior spectrometer in order to reduce the limitations imposed by the enormous line broadening of the previous setup. Furthermore, the variance in the polarization field caused by the manual polarization step was tackled by the construction of the shaking table and the design of the associated rider, using which the polarization step was performed at fixed distances from the spectrometer. Lastly, the implementation of a light sensor served to minimize and equalize the sample resting time between insertion into the magnet and execution of the NMR experiment.

Several drawbacks of the previous setup were, however, not resolved by the revised setup. While the line broadening effect was substantially reduced owed to an up to date shimming system and a probe matching the utilized sample tubes, line shape distortion and line broadening remained the predominant impediment in the examination of the recorded spectra (Figure 33). Both of these effects were caused by the insertion of the sample into the magnet, as the movement through the strong magnetic field induced small-scale magnetic variances, leading to a field inhomogeneity. Additionally, the settling of the sample tube caused vibrations in the probe, which added to the effects.

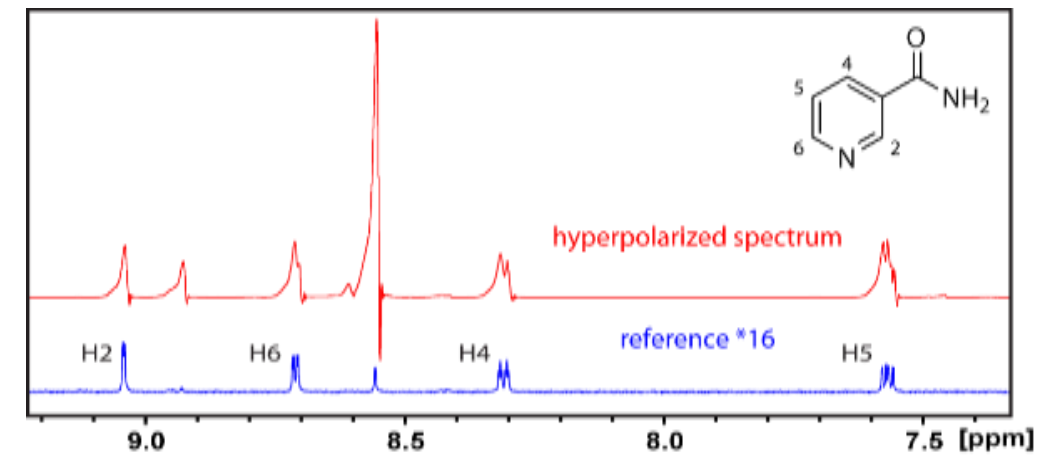

Figure 33: Exemplary spectrum overlay of the aromatic region of nicotinamide. For the hyperpolarized spectrum, the polarization step was performed at a field of $40 \mathrm{Gs}$. The reference spectrum is enlarged by a factor of 16 . Unlabeled resonances either originate from pyridine $(8.55 \mathrm{ppm})$ or from complex-coordinated substrates (8.95 ppm).

In SABRE experiments, the active catalyst usually binds several substrate molecules at a time, which occupy two distinguishable positions in the complex, leading to up to two additional proton resonances for each substrate proton resonance. These complex-bound substrates experience hyperpolarization in the same way as molecules that were already released from the complex, also gaining significant signal intensities. Additionally, residual pyridine resonances were present in the spectra, originating from incompletely deuterated pyridine molecules, either stemming from the precatalyst or their employment as a cosubstrate. Each of these resonances exhibited line shape distortion in the form of a downfield sloping effect that could span more than tenfold their line width at baseline level in the reference spectra, severely impeding signal separation. Furthermore, substrates only experiencing weak signal enhancement were at times overpowered by the prevalent pyridine resonances. 
Despite the mechanical polarization step being executed using the specifically designed sample mounting and the capability of fixing the distance from the NMR magnet, the magnitude of the magnetic field, at which the polarization step was performed, remained ambiguous. The intensity of the stray field was determined at the center of the sample mounting, yet owed to the steep field gradient, small variations of the distance to the magnet due to the length of the sample tube combined with the amplitude of the reciprocating motion of the mounting resulted in a substantial field gradient over the sample path (Figure 34). As the outer limits exhibited a greater distance from the spectrometer, the average polarization field was of lower magnitude than the determined field at the center of the retainer. This is consistent with the attained results, as the recorded field dependence curves display a slight shift to higher polarization fields when compared to previous results $[35,122]$.

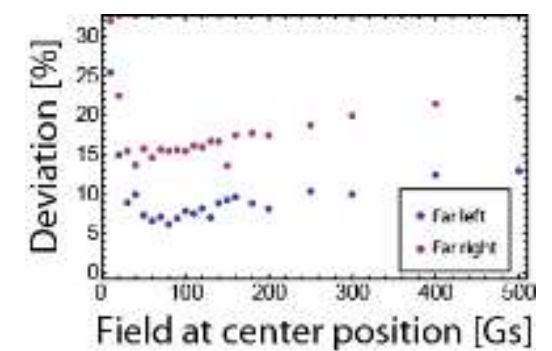

Figure 34: Deviations from the magnetic field determined at the center of the sample. The asymmetry is owed to the presence of the gas valve at the top end of the sample tube.

Additionally, as the polarization field strength in this setup was adjusted by changing the distance from the NMR magnet, the increments between the individual field strengths could not be kept equal. While at lower fields, the field gradient is flat and the distances between measurement points $10 \mathrm{Gs}$ apart were on the scale of several $\mathrm{cm}$, the gradient became exceedingly steeper for higher polarization fields. To reliably discern between the scale markings closer to the spectrometer, the step size of the polarization field had to be increased first to 20 , later to $50 \mathrm{Gs}$.

A further factor in the polarization field uncertainty stems from the possibility of still ongoing polarization transfer reactions after the shaking motion had stopped. This could be due to residual $p \mathrm{H}_{2}$ in the solution that had not yet been converted into $\mathrm{oH}_{2}$ or due to perturbations during the manual transfer, which caused a solvation of unused $\mathrm{pH}_{2}$ from the headspace over the solution. This is illustrated by observations made during experiments conducted at several higher polarization field strengths, for which the sample had to be moved around an obstacle in order to be inserted into the NMR magnet. Depending on the chosen sample path - passing in front of the object or behind it - the recorded spectra exhibited slightly divergent enhancement patterns.

While the implementation of the light sensor proved helpful in reducing some of the time variances occurring in the experiments, a main part of the experimental procedure was still executed manually. The removal of the sample from the mounting after the polarization step 
and the subsequent insertion into the spectrometer was not automatized and prone to timing variations. Furthermore, samples that were polarized at lower field were, due to the setup design, polarized at a greater distance from the spectrometer and therefor had to cover a greater distance to be inserted. This presumably resulted in higher losses of polarization during the transfer.

In addition to the drawbacks that changed the outcome of the experiments, the shaking table setup also exhibited shortcomings that hindered the conduct of the experiments itself. To achieve a proper mixing of the $\mathrm{pH}_{2}$ gas and the sample solution, the sample tube had to be shaken with certain vigor, taxing the entire equipment on the rider. The highly pressurized NMR tubes often burst due to the induced mechanical stress and even the metal parts of the crank yielded to the strain imposed on them. Lastly, even the electromotor had to be replaced due to the physical pressure.

Furthermore, determination of a complete field dependence proved a laborious and timeconsuming activity, as, on average, 45 polarizations experiments had to be performed manually, totaling several hours of physically straining, but highly repetitive tasks for each substrate.

In summary, the setup served to more accurately determine polarization field dependences for new and previously examined substrates. It further allowed examination of few substrates, for which the previous setup was insufficient. Still, performing the hyperpolarization experiments using this setup revealed several substantial flaws that had to be addressed and improved on for the following experimental design.

\subsubsection{Experimental Setup 2}

The Bruker Para-Hydrogen Polariser and the associated flow injection probe were successfully taken into operation and produced spectra of hyperpolarized compounds. After only a short time, however, sample transfers became increasingly unreliable and the default rate impeded satisfactory conduct of the hyperpolarization experiments. Efforts were undertaken to adjust the transfer parameters, exposing a strong discrepancy between the parameters necessary to fill the dummy flow cell and those for the actual probe. As a result, the transfer parameters for the probe had to be optimized solely relying on line shape comparison with a reference spectrum and without visual control of the liquid level. As the adjustments using this method were made with exceedingly greater care, the transfer times, once stable transfers had been achieved, significantly exceeded the optimal values. Over time, the transfer parameters were adjusted on a number of occasions without reaching an ultimately satisfying set, which combined fast, reliable and reproducible transfers. Subsequently, hyperpolarization experiments were conducted using a number of different transfer speeds and times. With longer transfer times, however, the amount of hyperpolarization preserved for read-out in the spectrometer is reduced, leading to strongly varying enhancement factors attained for different sets of transfer parameters. The 
experimental results presented here, which were recorded over an extended period of time, are therefore not fully comparable with respect to the achieved enhancement factors.

Furthermore, owed to the significantly increased sample volume, the absolute catalyst and sample concentration was halved compared to the shaking table setup in order to reduce consumption of the catalyst. As a result, a reduction in signal enhancement was expected. Also, the co-substrate approach to sample preparation was abandoned, as this simplified the preparation process and examination of relative enhancements was of a higher priority compared to absolute magnitude of the enhancement.

\subsubsection{Substrates examined using the Ir(COD)(Py-d5)(PCy-d $\left.\mathrm{d}_{33}\right)$ precatalyst}

Initial experiments using the newly installed setup were conducted on already examined substrates in order to cross-validate the Polariser and the previously recorded data for comparability.

\section{3-Methylpyrazole, pyridine and 3-phenylpyrazole}

The results for 3-methylpyrazole (Figure 35) and pyridine (Figure 36) showed a good agreement with preceding experiments when plotted with the effective fields derived from linear regression. The maximum enhancements are again in the regions around 40 and 140 Gs for all protons except for the pyridine meta protons, which, as usual, exhibit a wider span of highest enhancement in the lower half of the examined field range.

Results for 3-phenylpyrazole (Figure 37) also show a good agreement with previous data for the protons in positions 4 and 5 . In addition, it was possible to observe and analyze enhancement for the phenyl ring protons which had previously been prevented by the inferior resolution provided by the two prior setups. Owed to the distance from the donor atom, the amount of polarization transferred to these nuclei is expectedly low with a maximum enhancement of just above a factor of 5 for the ortho protons. Interestingly, these protons show an inverted field dependence profile compared to the pyrazole ring protons, similar to ethoxy protons in 4-ethoxy-3-methylpyrazole (Figure 25). The meta and para protons exhibit a further anomaly, exhibiting only positive maxima at both 40 and $140 \mathrm{Gs}$.

As the mixing chamber is positioned at a magnetic field of between 100 and $130 \mathrm{Gs}$, the effective field can be modulated to give negative values when an induced negative field overcompensates the residual field. This effectively inverts the direction of the magnetic field, yet without consequences for the polarization transfer process. As the amount of transferred polarization is only dependent on the magnitude, but not the direction of the field, a symmetrical distribution is expected around the averaged "zero field", which is clearly visible in the field dependence graphs. Close examination reveals the line of symmetry not being identical with the plotted $0 \mathrm{Gs}$ line but shifted by approx. $5 \mathrm{Gs}$ into the negative range. This is owed to polarization transfer occurring not only at a single distinct polarization field strength, but due to a field gradient over the sample volume, a spread of field strengths contributes to the average polarization field, which renders the accurate 
determination of the magnetic field difficult. This effect is further discussed in section 3.1.2.4.

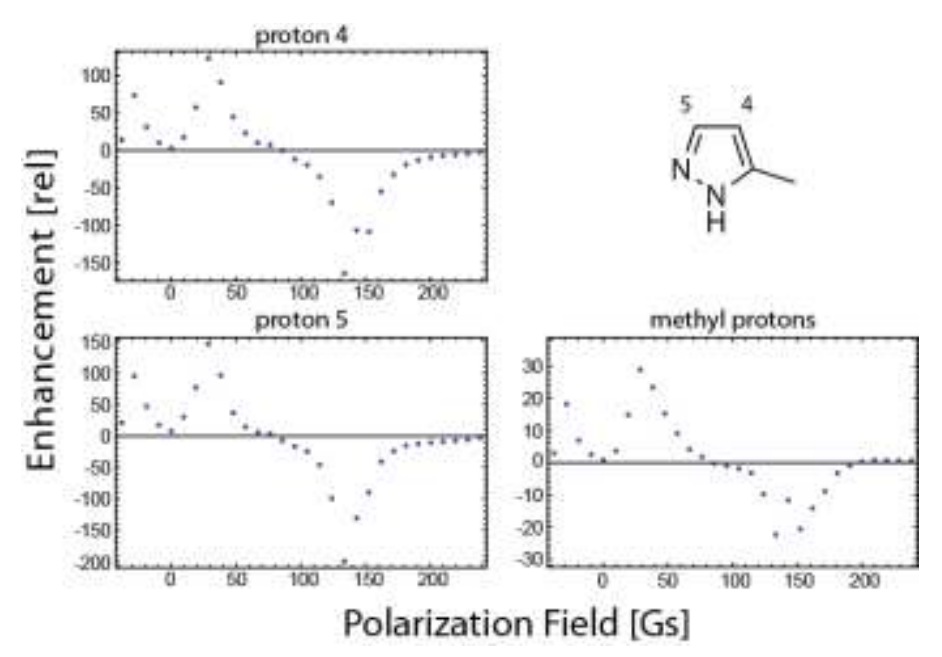

Figure 35: Polarization field dependence of signal enhancement for the protons in 3-methylpyrazole. The molecular structure of the substrate molecule is given at the top right.

It must further be noted that due to the earth's magnetic field and the above-mentioned stray field gradient, the effective polarization field achieved by application of the corresponding modulation field value is not truly 0 Gs but corresponds to the lowest achievable spread of field strengths attainable for this setup, which is approximately centered around the zero point.

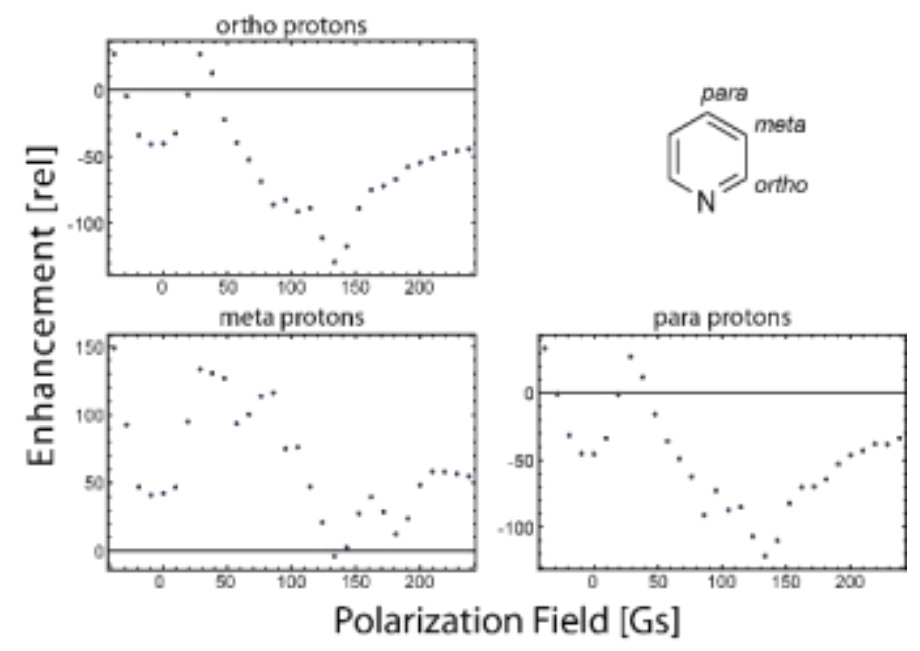

Figure 36: Polarization field dependence of signal enhancement for the protons pyridine. The molecular structure of the substrate molecule is given at the top right.

Overall, the smoothness of the data in Figure 37, especially of the pyrazole ring protons, formidably illustrates the level of consistency achievable with the Para-Hydrogen Polariser setup when the sample is reliably transferred into the flow injection probe. 

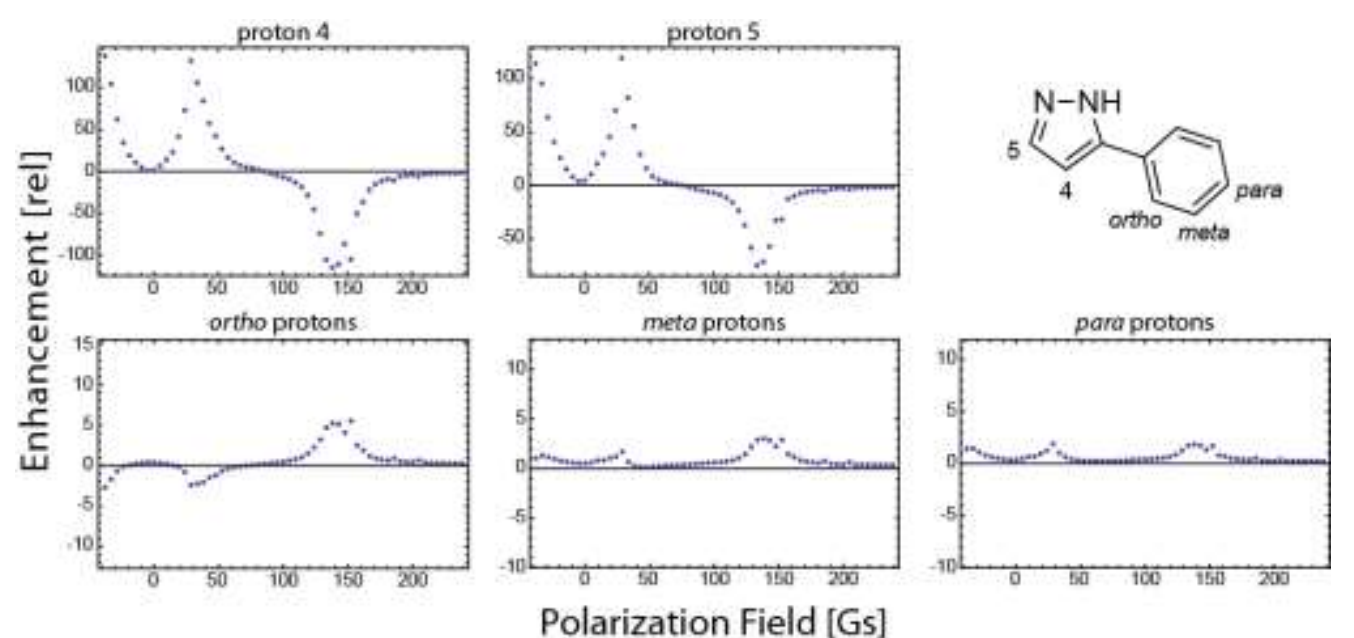

Figure 37: Polarization field dependence of signal enhancement for the protons in 3-phenylpyrazole. The molecular structure of the substrate molecule is given at the top right.

\section{1,2,4-Triazole}

The results determined for 1,2,4-triazole using the Polariser unit for the first and only time showed a considerable deviation from the manually determined enhancement patterns (Figure 38). While the previously obtained results showed a relatively confuse enhancement profile presumably caused by superposition of divergent enhancement profiles (comp. 3.1.1.1) and a characteristic sudden sign change at a field of $50 \mathrm{Gs}$, this denoting feature was not observable with the Polariser setup.

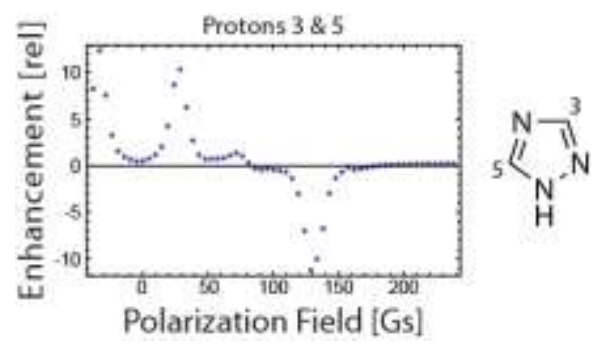

Figure 38: Polarization field dependence of signal enhancement for the protons in 1,2,4-triazole. The molecular structure of the substrate molecule is given on the right.

An attempt of an explanation can be made by regarding the differences in field deviations for the two setups. For the shaking table, the field variations were owed to the length of the shaking path, as its ends had a greater distance from the NMR magnet compared to the center and therefore experienced a lower magnetic field. These deviations were, as was shown above, in the range of 15 to $19 \%$ for most fields, depending on the steepness of the field gradient at the sample position (Figure 34). At the distance of the $50 \mathrm{Gs}$ marking, the field at which the sign change was observed, the deviation was determined to be on the lower side of the spectrum with $15 \%$, corresponding to $7.5 \mathrm{Gs}$. As the scale marking denotes the highest magnetic field in the shaking path, the effective field range for this polarization step was between 42.5 and $50 \mathrm{Gs}$, neglecting possible hyperpolarization during the sample transfer through the stray field. 
For the Polariser setup, the field gradient across the sample volume is significantly steeper, as the length of the mixing chamber runs directly along the $z$-axis of the NMR stray field. Without employment of the solenoid coil, a field variance of $23 \mathrm{Gs}$ was determined for three distinct points inside the cavity which holds the mixing chamber. Additionally, due to a noticeable deviation from the field homogeneity, the modulation at the top part of the coil cavity shows a reduced efficiency, which on the one hand decreases the variance for higher fields, but on the other hand increases it for lower field strengths (comp. 4.1.4.3). For an effective field of $50 \mathrm{Gs}$ at a depth of $7 \mathrm{~cm}$, the field variance across the three characteristic points amounts to $27 \mathrm{Gs}$, corresponding to a percentage deviation of $54 \%$. Excluded from this value is the sample volume below the $7 \mathrm{~cm}$ marking, which further adds to the deviation.

In the manually recorded spectra, the field range at which the characteristic sign change occurred was extremely narrow, displaying a change from 80 fold enhancement at $40 \mathrm{Gs}$, the largest positive signal gain, to a value of -175 at $50 \mathrm{Gs}$, representing the highest enhancement overall for this substrate, within only one field increment of $10 \mathrm{Gs}$ (comp. 3.1.1.1, Figure 27). Within another field increment, the enhancement decreases to -28 at 60 Gs before returning to positive enhancement values.

During the $50 \mathrm{Gs}$ polarization step in the Polariser setup, the sample is at the least distributed over effective fields ranging from 50 to $77 \mathrm{Gs}$, all of which contribute to the average effective polarization field. Additionally, further, lower field strengths also contribute in the polarization step, resulting in a complex average polarization field, amounting to no net enhancement at a Polariser setting of $50 \mathrm{Gs}$.

\section{Indazole}

The annular pyrazole derivative indazole had also been examined using the shaking table setup and exhibited very distinct maximum enhancements at 40 and $140 \mathrm{Gs}$ (Figure 39). For the remaining polarization fields, the graphs exhibited a flat characteristic as opposed to a fluent transition between the two maxima as often observed for different pyrazole and pyridine derivatives. The newer results verify the previous data and additionally show smaller, secondary maxima at fields above $200 \mathrm{Gs}$, which were previously not resolved due to the increment size at higher fields. The relatively low signal enhancements compared to those attained with the shaking table setup, using which a 100 -fold signal gain was achieved for proton 3 at $40 \mathrm{Gs}$, is attributed to slow sample transfer speeds that were employed at this stage. 

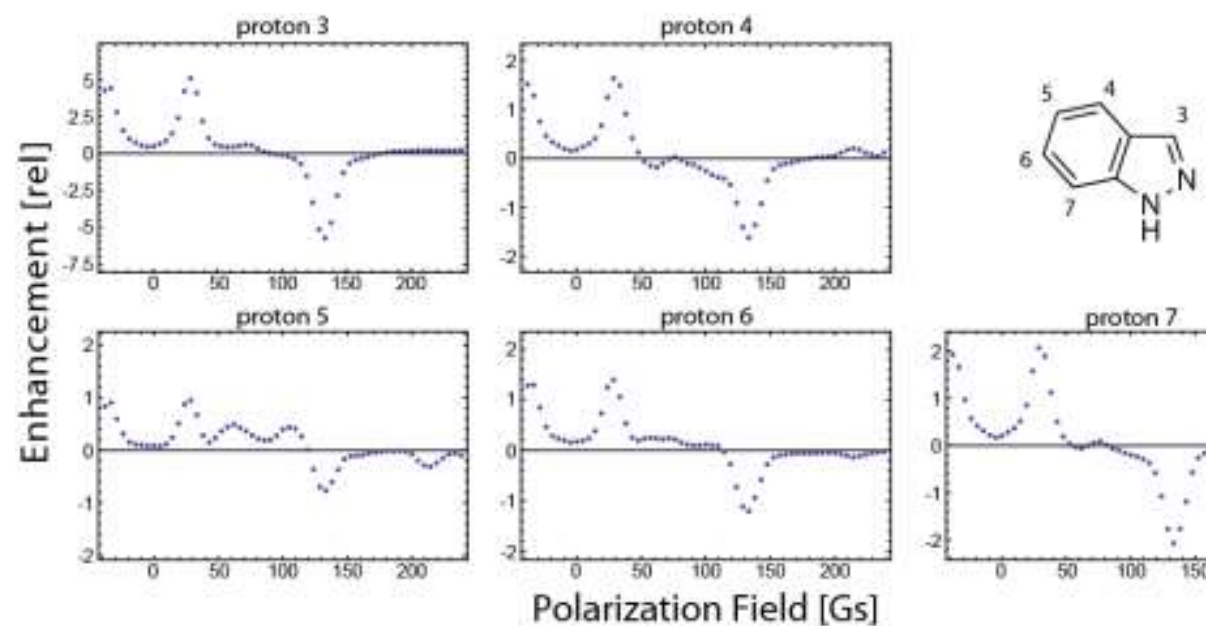

proton 7

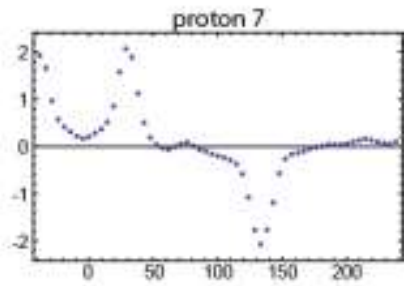

Figure 39: Polarization field dependence of signal enhancement for the protons in indazole. The molecular structure of the substrate molecule is given at the top right.

\section{4-Ethoxy-3-methylpyrazole}

Results obtained for 4-ethoxy-3-methylpyrazole are depicted in Figure 40, validating the previously observed inversion of the enhancement pattern for the protons in the ethoxy group (Figure 25), presumably owed to scalar coupling constants between nuclei, using which the polarization is redistributed within the substrate molecules.

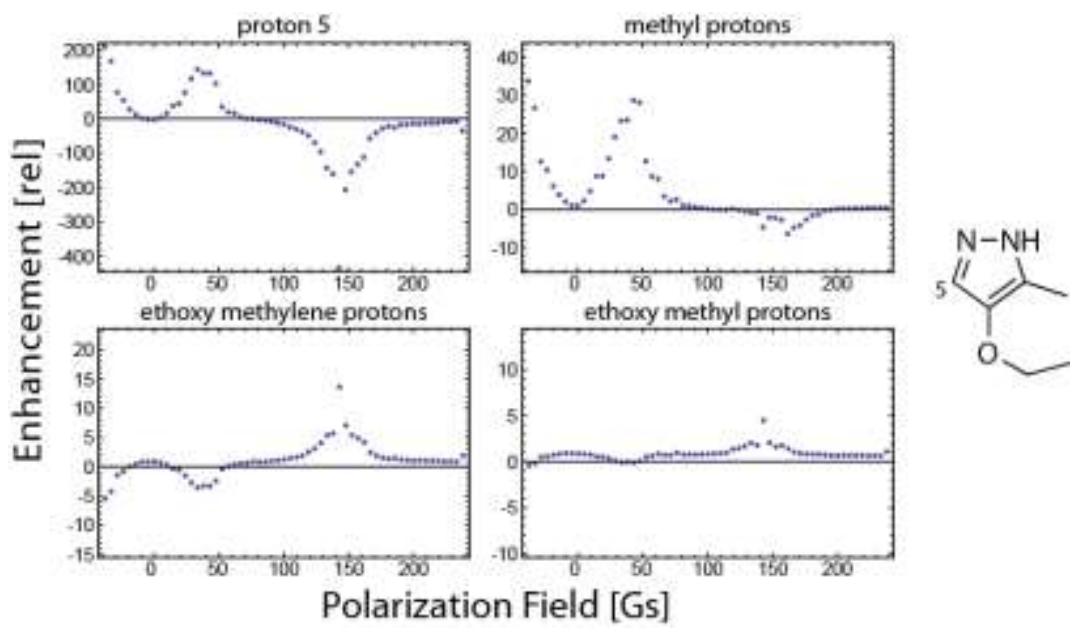

Figure 40: Polarization field dependence of signal enhancement for the protons in 4-ethoxy-3-methylpyrazole. The molecular structure of the substrate molecule is given on the right.

\section{2,6-Dideuteropyridine and nicotinamide}

Results for 2,6-dideuteropyridine (Figure 41) and nicotinamide (Figure 42) also correspond to the previously determined field dependences. Contrary to the results for 1,2,4-triazole (Figure 38), the similar abrupt sign change at around $50 \mathrm{Gs}$ is resolved for the former substrate, presumably owed to the broader field range at which the effect occurs and the continued negative enhancement for the subsequent higher fields. 


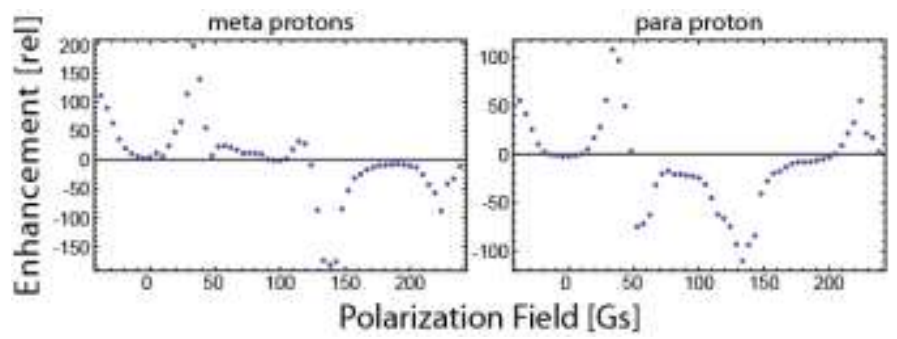<smiles>[2H]c1cccc(C)n1</smiles>

Figure 41: Polarization field dependence of signal enhancement for the protons in 2,6-dideuteropyridine. The molecular structure of the substrate molecule is given on the right.

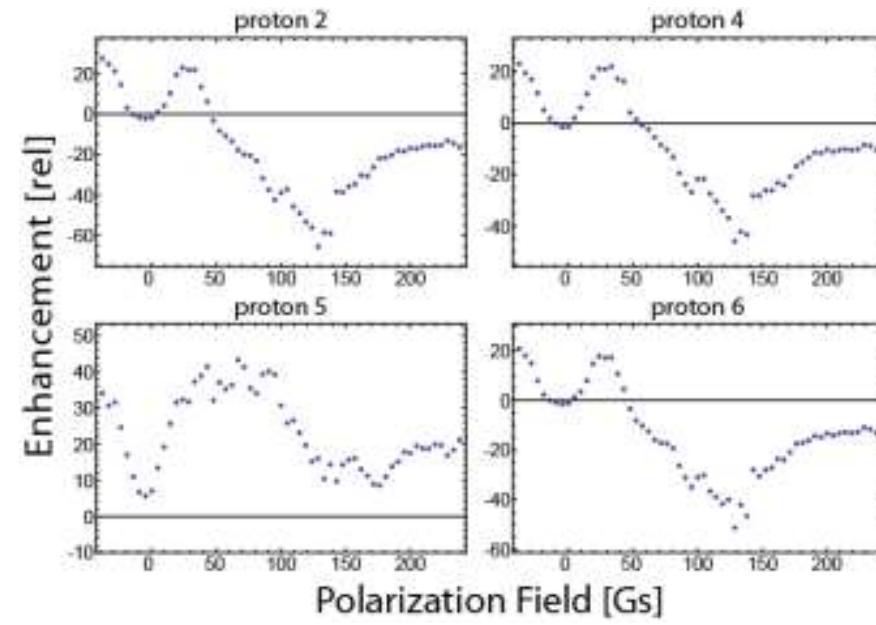<smiles>NC(=O)c1cccnc1</smiles>

Figure 42: Polarization field dependence of signal enhancement for the protons in nicotinamide. The molecular structure of the substrate molecule is given on the right.

\section{Quinoline}

In Figure 43, the signal enhancement curves exhibit a new pattern unlike what was expected for a molecule with a pyridine substructure. Basically, two different patterns are distinguishable: Protons 4,5 and 8 gain enhanced positive signal intensities with a rather broad maximum at fields of just below $100 \mathrm{Gs}$. For protons 3, 6 and 7, the enhancement has a negative sign and the distinct maximum is at a field of $50 \mathrm{Gs}$. Proton 2, for which highest enhancements were expected, as it is closest to the donor atom, exhibits what resembles a superposition of these two patterns, resulting in signal intensities lower than the Boltzmann magnetization for all polarization fields. 


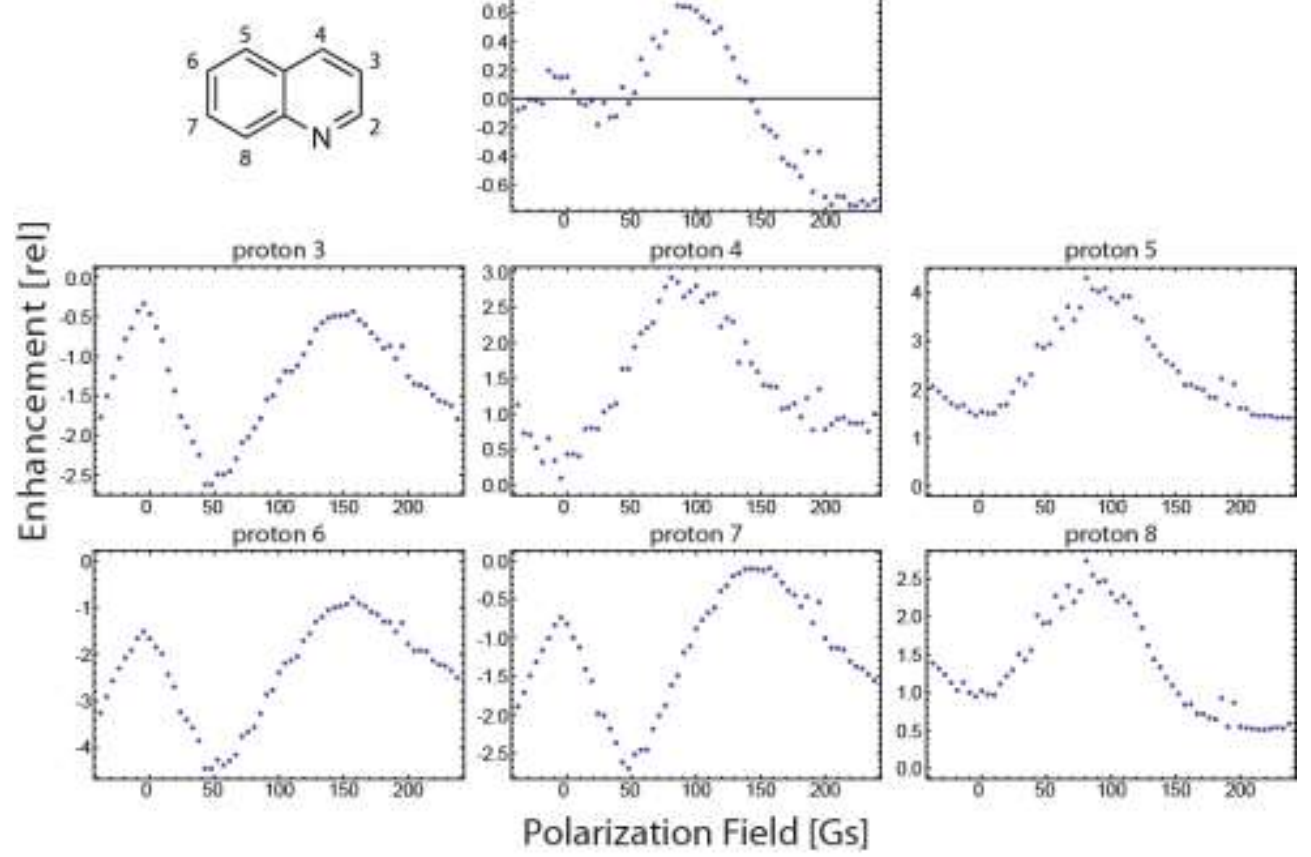

Figure 43: Polarization field dependence of signal enhancement for the protons in quinoline. The molecular structure of the substrate molecule is given at the top left.

\subsubsection{Substrates examined as a comparison of PCy,-, IMes- and SIMes- containing catalysts}

In 2011, Cowley et al. reported a more efficient transfer of polarization using an iridium-based catalyst containing the IMes ligand [34]. In order to evaluate differences in the field dependence of SABRE to the at that point employed $\mathrm{PCy}_{3}$-containing catalyst, the respective precatalyst $[\operatorname{Ir}(C O D)(I M e s) C l]$ as well as its SIMes-containing analog $[\operatorname{Ir}(\mathrm{COD})(\mathrm{SIMes}) \mathrm{Cl}]$ were synthesized. The following substrates were examined using these three different catalysts. The experimental series for each substrate were conducted sufficiently close in time and using identical transfer parameters to ensure comparability.

\section{4-Methylpyrazole}

In Figure 44, a difference in the field dependence for the different catalyst is readily observable. Using the $\mathrm{PC}_{3}$-containing catalyst, the expected and previously reported behavior with maxima around 30 and $140 \mathrm{Gs}$ is reproduced for both the ring protons and the methyl group. With the IMes and SIMes ligands, only one very distinct maximum is observable at a field of 50 to $60 \mathrm{Gs}$ and only negligible polarization is achieved for fields greater than $100 \mathrm{Gs}$. This finding is in excellent agreement with the predicted field dependences by Pravdivtsev et al. [37].

Furthermore, the IMes complex facilitates a considerably more efficient polarization transfer, generating four times as much signal intensity compared to the SIMes complex and surpasses Crabtree's catalyst by a factor of two. 

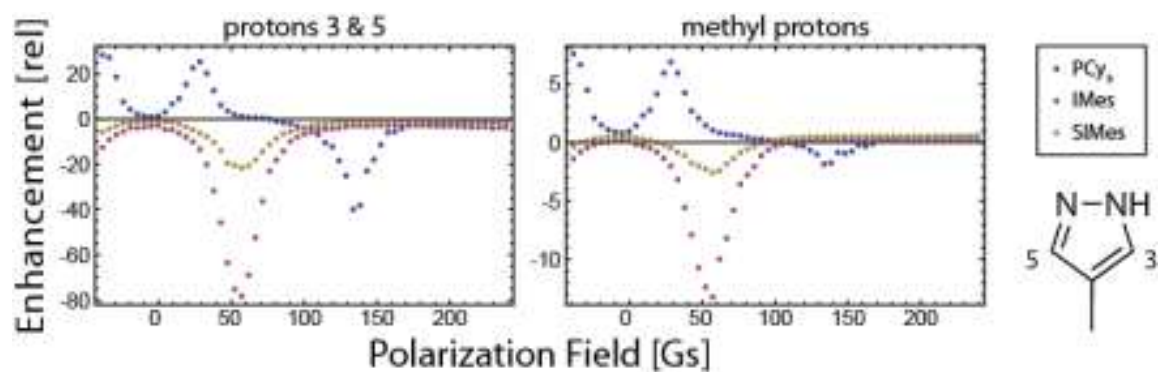

Figure 44: Polarization field dependence of signal enhancement for the protons in 4-methylpyrazole determined with various catalysts. The molecular structure of the substrate molecule is given on the right.

\section{3,5-Dimethylpyrazole}

Figure 45 displays the results obtained for 3,5-dimethylpyrazole, which indicate a lower tolerance for bulky substrates when employing the $[\mathrm{Ir}(\mathrm{COD})(\mathrm{IMes}) \mathrm{Cl}]$ precatalyst. While the $\mathrm{PC}_{3}$ complex facilitates polarization transfer at a low efficiency but resulting in a familiar pattern, the IMes-containing catalyst fails at the transfer of polarization to the disubstituted pyrazole. Polarization of the even bulkier 3,5-diphenylpyrazole was, as for Crabtree's catalyst, also not achieved using the IMes catalyst.

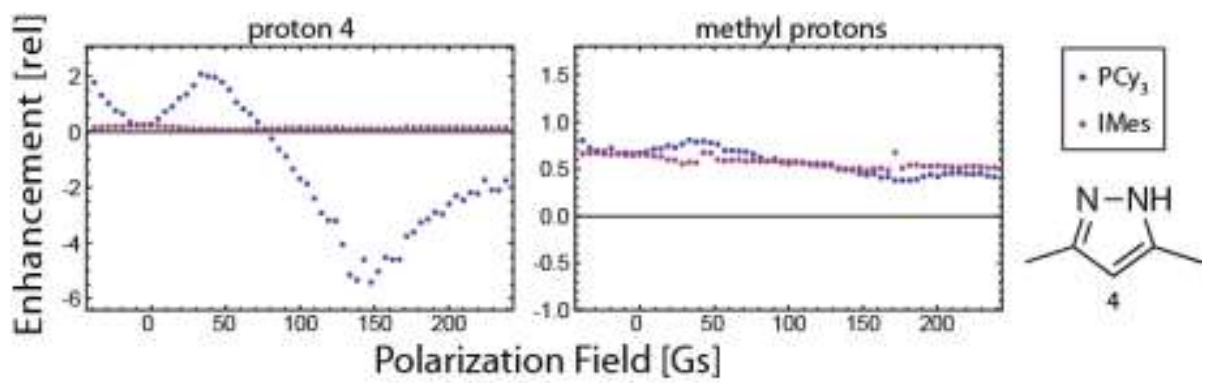

Figure 45: Polarization field dependence of signal enhancement for the protons in 3,5-dimethylpyrazole determined with two different catalysts. The molecular structure of the substrate molecule is given on the right.

\section{3-Methylpyridine}

Figure 46 again depicts the superiority of the IMes catalyst over its competitors in the hyperpolarization of 3-methylpyridine. Across all resonances, it tops the SIMes catalyst by a factor of 4 and surpasses the $\mathrm{PCy}_{3}$ complex for protons 2, 4 and 6 by a factor of 2 . Only for proton 2 and the methyl protons, Crabtree's catalyst can almost match the enhancement achieved with the IMes catalyst. Maximum enhancements for the NHC catalysts are achieved at similar fields of approx. $60 \mathrm{Gs}$ with a slight shift to stronger fields for the SIMes ligand. The exhibited maxima are considerably broader than for the previously examined pyrazole substrate with substantial signal enhancements achieved over the entire range of polarization fields. For low fields of below $30 \mathrm{Gs}$ and high fields over $160 \mathrm{Gs}$, proton 5 and to a lesser extent - the methyl protons even experience signal enhancement with a positive phase. Results obtained with Crabtree's catalyst correspond to the previously examined enhancement patterns for the 3-substituted pyridine derivative nicotinamide. 

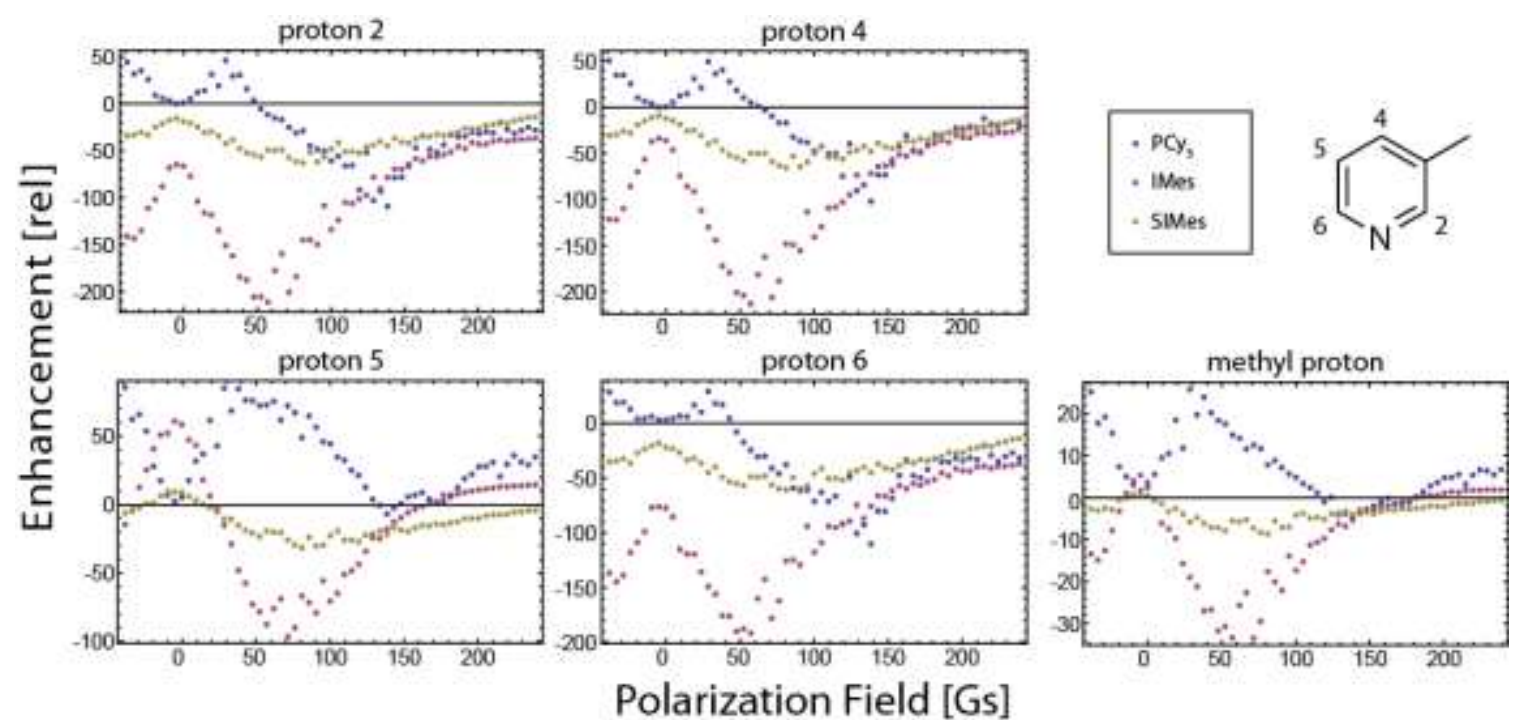

Figure 46: Polarization field dependence of signal enhancement for the protons in 3-methylpyridine determined with various catalysts. The molecular structure of the substrate molecule is given at the top right.

\section{3-Methoxypyridine}

3-Methoxypyridine, the analog of the previous substrate with an oxygen inserted into the $\mathrm{C}-\mathrm{C}$ bond between heterocycle and methyl group, exhibits similar enhancement profiles for the ring protons (Figure 47). The IMes containing complex again facilitates the highest level of polarization transfer, slightly surpassing the enhancements for 3-methylpyridine. Enhancements achieved with the SIMes ligand also increased, yet the catalyst still falls short of the IMes ligand by factors of 2 to 3 . For $\mathrm{PC}_{3}$, the results are of comparable magnitude. The field dependence profiles for all three catalysts in regard to maxima location are comparable to those of the previous substrate for the ring protons.
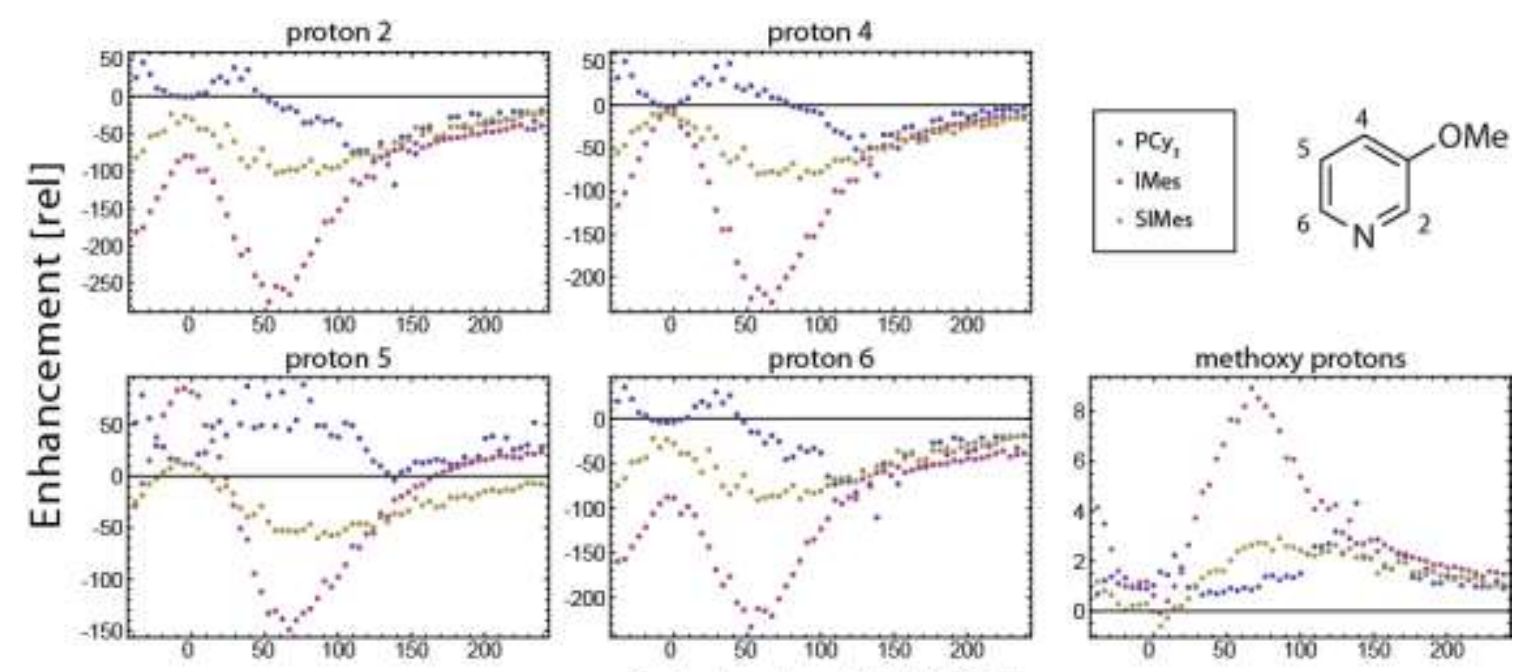

\section{Polarization Field [Gs]}

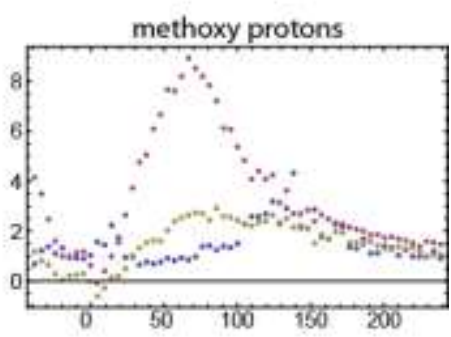

Figure 47: Polarization field dependence of signal enhancement for the protons in 3-methoxypyridine determined with various catalysts. The molecular structure of the substrate molecule is given at the top right.

For the methoxy protons, the enhancement is significantly reduced compared to the methyl protons in 3-methylpyridine; in the case of the IMes ligand, which gains the highest 
enhancement in both cases, by a factor of almost 4 . The largest gain in signal intensity was in this case achieved at $70 \mathrm{Gs}$, which marks a slight shift to higher fields compared to the ring protons, which gain their highest intensities at fields around $60 \mathrm{Gs}$.

Most notable is the fact that the methoxy protons experience almost exclusively positive enhancements using all three catalysts, which marks a complete inversion for the NHC catalysts compared to the results for 3-methylpyridine. Similar behavior was previously observed for the protons of the ethoxy group in 4-ethoxy-3-methylpyrazole when examined with the $\mathrm{PC}_{3}$ catalyst. Hyperpolarization of the methoxy group using the latter catalyst was achieved with rather mediocre results with regard to signal intensity. The obtained polarization field dependence exhibits, however, a slightly deviant profile, which is not simply inverted, like was the case for the mentioned ethoxy protons, but displays two positive maxima. Additionally, the first maximum appears at an uncommonly low field of only 15 Gs.

Reversible binding to the polarization transfer catalyst is a key element in SABRE hyperpolarization. Too tight of a binding reduces the turnover rate, limiting the number of molecules that can be polarized in a given time interval. On the other hand, too little binding affinity can prevent a sufficient interaction between the $\mathrm{pH}_{2}$ nuclei and the substrate molecules, preventing efficient polarization transfer $[40,81]$. It is therefore of great importance that molecules of interest remain on the middle ground between these two extremes. Despite their difference in nucleophilicity owed to the $+I$ and $-I$ effects of the methyl and methoxy groups, respectively, and the associated expected difference in binding affinity, the magnitude of enhancement was of surprisingly similar magnitude for both substrates. It was therefore of interest to examine if a modulation of the electron density within the heterocycle and consequently on the donor atom via variation of the substituents would directly translate into a modulation of the achieved enhancement levels. Therefore, five additional 3-substituted pyridines were examined.

\section{3-Hydroxypyridine}

The results for 3-hydroxypyridine displayed in Figure 48 show the most substantial deviation from the previous results, as that the SIMes containing catalyst, which was previously clearly outperformed by the IMes catalyst, is able to match up to and even surpass the enhancement levels achieved using the latter complex for selected resonances. For Crabtree's catalyst, the enhancement is relatively low, achieving polarization of roughly half the intensity compared to the previous two substrates. Interestingly, the results for the IMes catalyst seem to be unaffected. Such a substantial change in results compared to 3-methoxypyridine was not expected for the SIMes and $\mathrm{PCy}_{3}$ catalysts, as the basicity of the two substrates is of comparable strength (Table 1). 3-Hydroxypyridine is, however, capable of tautomerism, rapidly changing between a neutral and a zwitterionic form, in which the hydroxy proton is transferred onto the nitrogen and which therefore exhibits no basicity at the nitrogen site. The $\mathrm{N}$-site of the neutral form therefore exhibits a considerably larger basicity, as indicated by the larger pKa value of 5.20 [125] compared to that determined for 
the mixture of the neutral and the dipolar form present in aqueous solution, which is between 4.80 and 4.86 [126]. The conversion between the two forms is swift compared on the NMR timescale, so that the individual tautomers are not resolved [127]. The polarization field strengths at which the most efficient transfer of polarization is achieved are not affected by this effect.

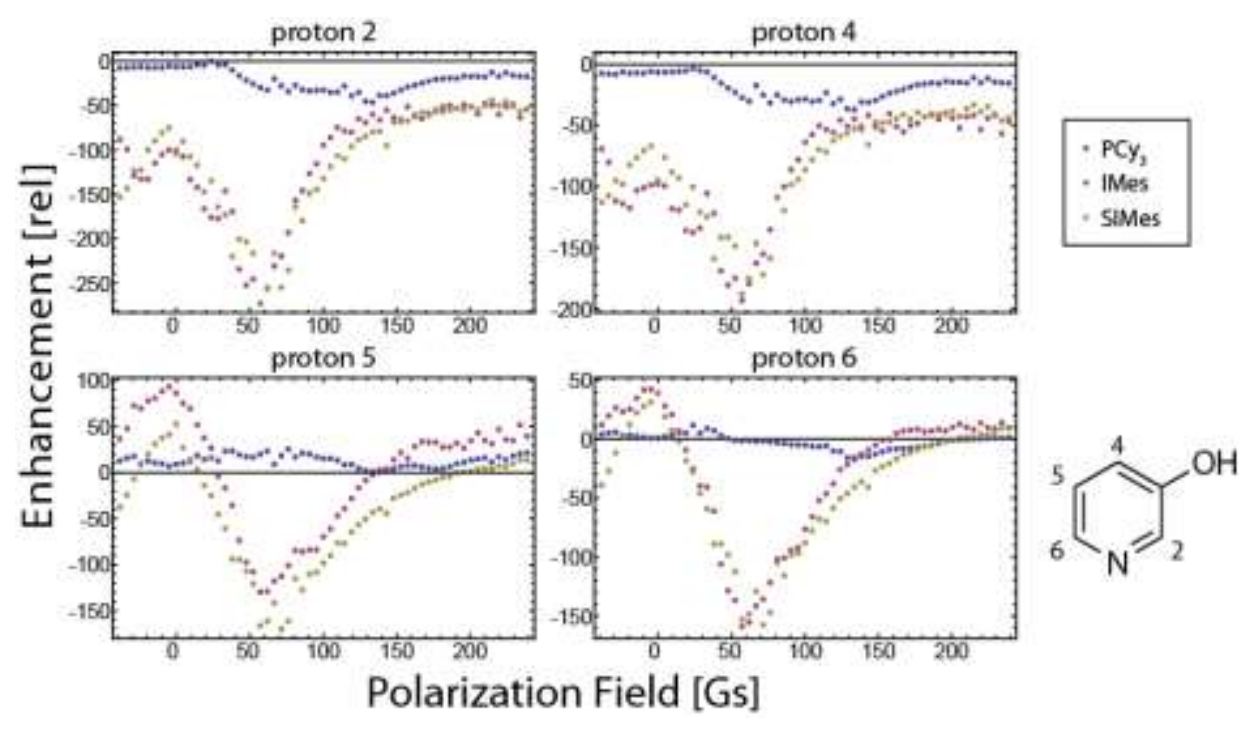

Figure 48: Polarization field dependence of signal enhancement for the protons in 3-hydroxypyridine determined with various catalysts. The molecular structure of the substrate molecule is given on the right.

\section{3-Fluoropyridine}

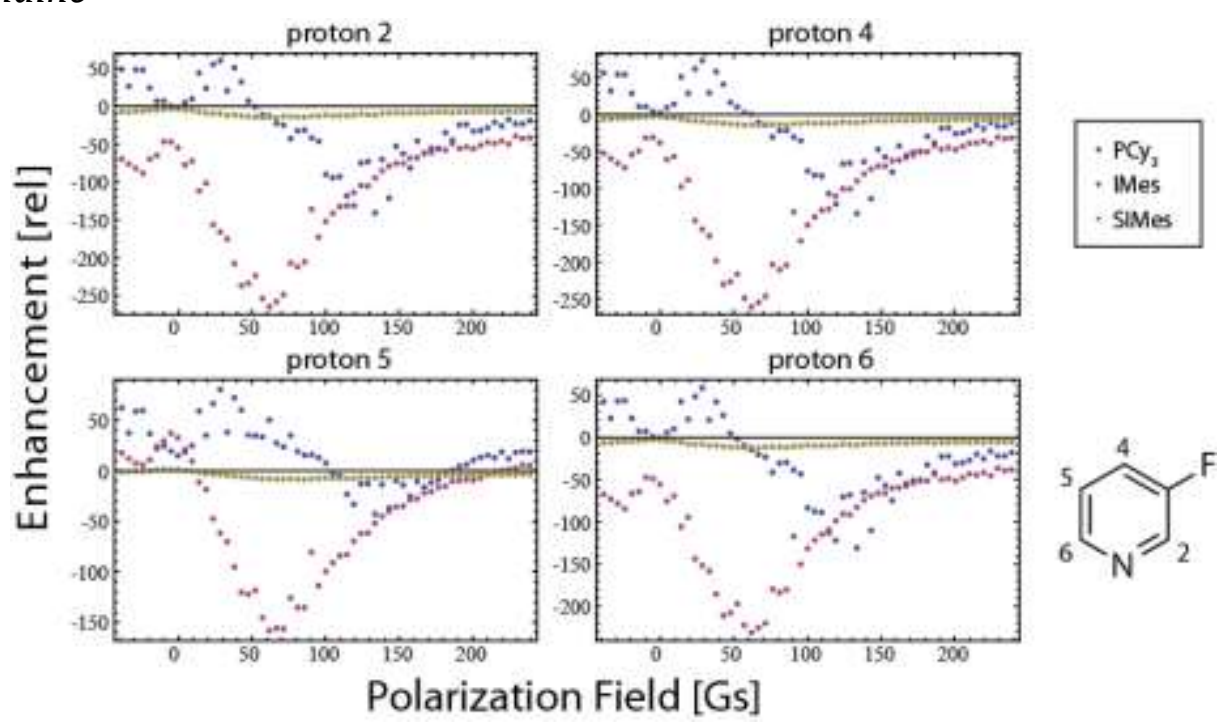

Figure 49: Polarization field dependence of signal enhancement for the protons in 3-fluoropyridine determined with various catalysts. The molecular structure of the substrate molecule is given on the right.

In 3-fluoropyrdine, the electron density in the heterocycle is reduced due to the strong electronegativity exhibited by the fluorine. Using the $\mathrm{PC}_{3}$ catalyst, enhancement factors of over 130 were achieved for protons 2, 4 and 6 (Figure 49), surpassing the results for the previous three substrates. The IMes catalyst once more excels over the competitors, showing superior enhancement on par with the results for 3-methoxypyridine. For the SIMes 
catalyst, however, the employment of the halogen substituent proves detrimental, as only very limited hyperpolarization is achieved, falling short of all previous results for pyridine derivatives.

\section{3-Chloropyridine}

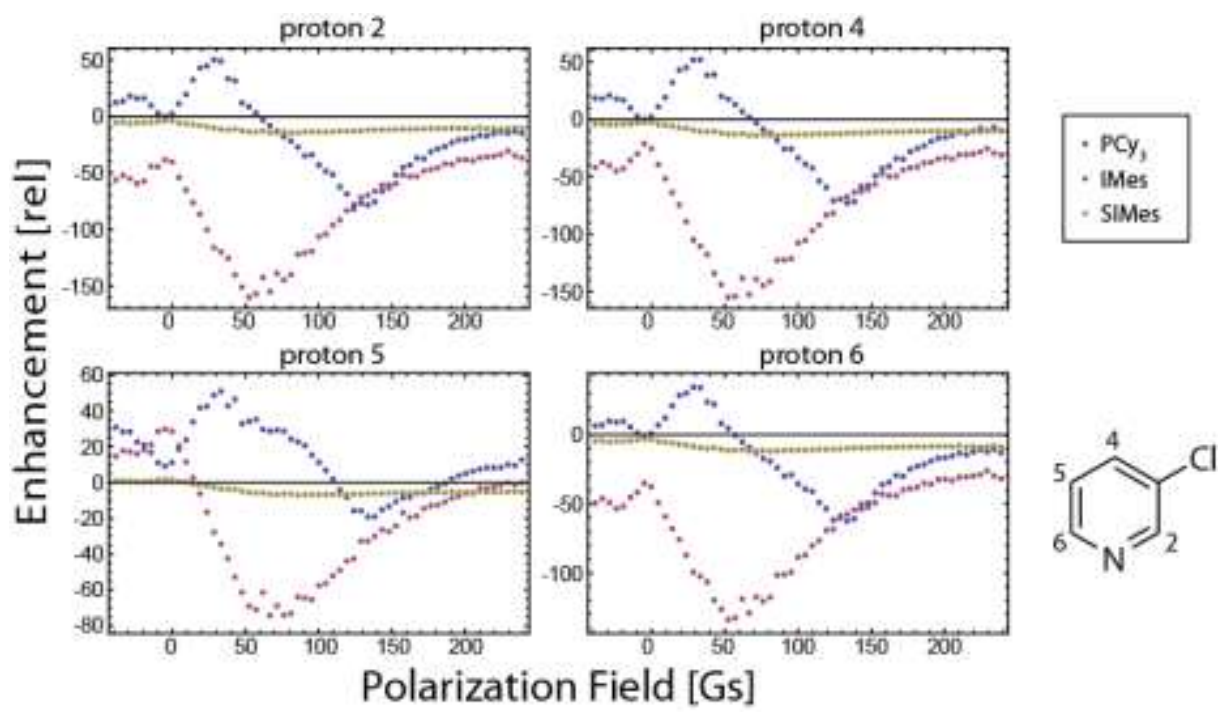

Figure 50: Polarization field dependence of signal enhancement for the protons in 3-chloropyridine determined with various catalysts. The molecular structure of the substrate molecule is given on the right.

Results for 3-chloropyridine are similar for the SIMes ligand (Figure 50), showing low enhancements which are comparable to the previous substrate. The IMes and $\mathrm{PCy}_{3}$ catalyst both experience a drop in enhancements for this substrate to about 50 to $60 \%$ of the values achieved for the previous compound.

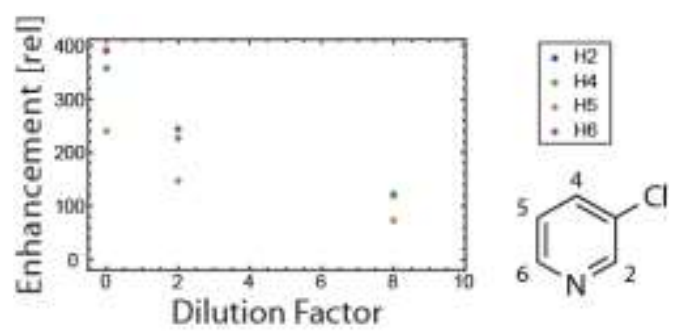

Figure 51: Maximum enhancements of the protons in 3-chloropyridine achieved using $\operatorname{the}[\operatorname{Ir}(\mathrm{IMes})(\mathrm{COD}) \mathrm{Cl}]$ precatalyst in dependence of the level of dilution.

A quick dilution experiment using the IMes catalyst and 3-chloropyridine as a substrate indicated an exponentially decaying dependence on the overall concentration (Figure 51). This was to be expected for a catalyst-mediated interaction between two species [51].

\section{3-Bromopyridine}

Results for 3-bromopyridine show a slight increase in signal intensity achieved by the SIMes ligand, yet the absolute enhancement remains low (Figure 52). For the IMes complex, results also increased compared to the previous substrate, but remain below those of the initial four pyridines. Furthermore, the field range for maximum enhancement appears to be slightly broadened for this compound yet remaining in the accustomed region. Crabtree's 
catalyst is able to increase the achieved level of hyperpolarization and experiences the highest enhancement factors of this experimental series for 3-bromopyridine. For protons 2 , 4 and 6 it almost matches up the IMes complex and even surpasses it for proton 5.

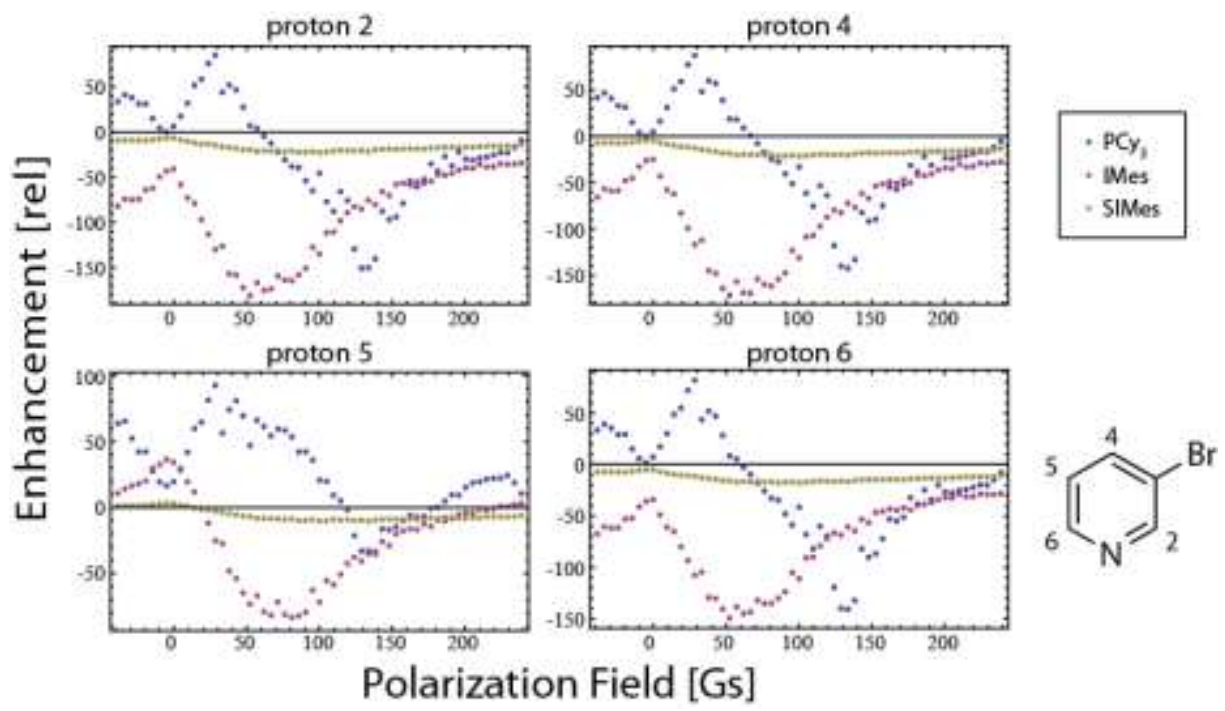

Figure 52: Polarization field dependence of signal enhancement for the protons in 3-bromopyridine determined with various catalysts. The molecular structure of the substrate molecule is given on the right.

\section{3-Iodopyridine}

For 3-iodopyridine, the NHC catalysts both remain approximately at the enhancement level achieved for the bromo-substituted analog (Figure 53). Crabtree's catalyst forfeits part of its enhancement, only achieving half of the level of polarization for protons 2, 4 and 6 compared to its IMes counterpart. Once more, the maxima in the field dependences remain in their accustomed ranges.

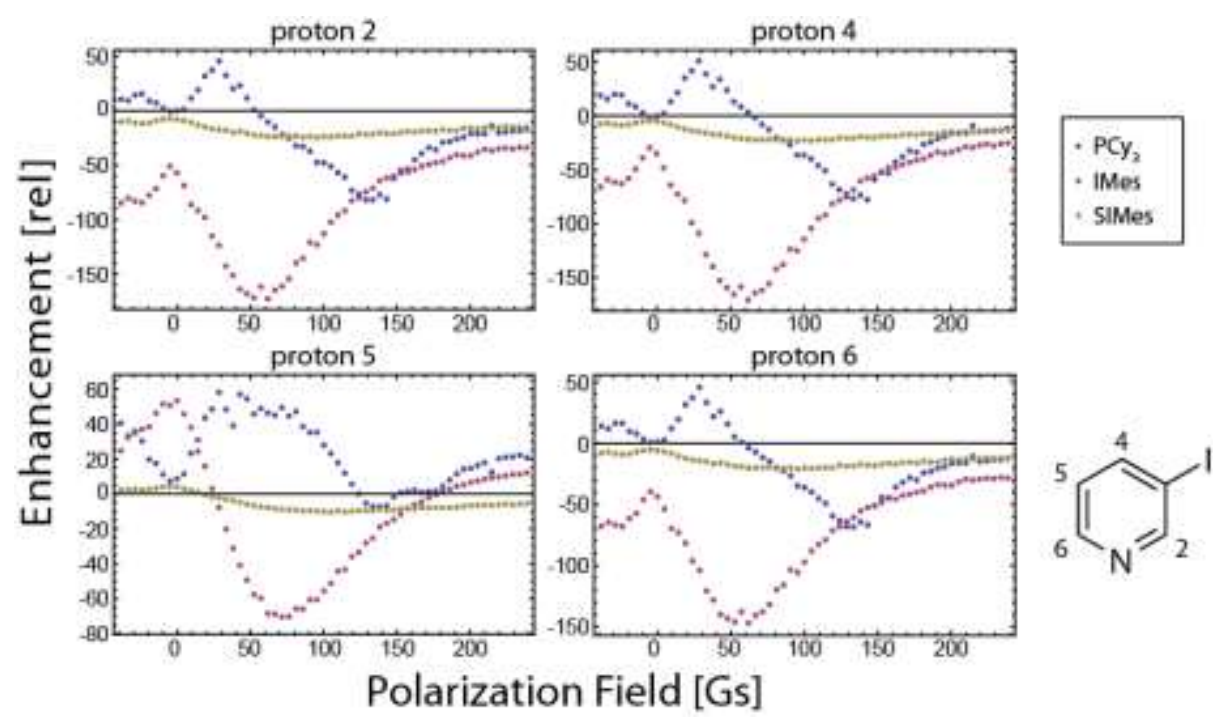

Figure 53: Polarization field dependence of signal enhancement for the protons in 3-iodopyridine determined with various catalysts. The molecular structure of the substrate molecule is given on the right. 


\section{3,5-Dichloropyridine}

Further reduction of the basicity by attaching two chloro substituents to the pyridine molecule had across-the-board highly adverse effect on the level of achieved hyperpolarization (Figure 54). While the IMes catalyst still facilitated a decent level of polarization transfer, surpassing that attained by the other catalysts for various previous substrates, the results for 3,5-dichloropyridine denote the lowest levels accomplished for any substrate examined in this comparison series. The polarization fields at which the highest enhancements were achieved is not significantly altered. For Crabtree's catalyst, however, the maximum at higher fields is considerably broadened, remaining on a lightly decreasing level across the entire higher field range.
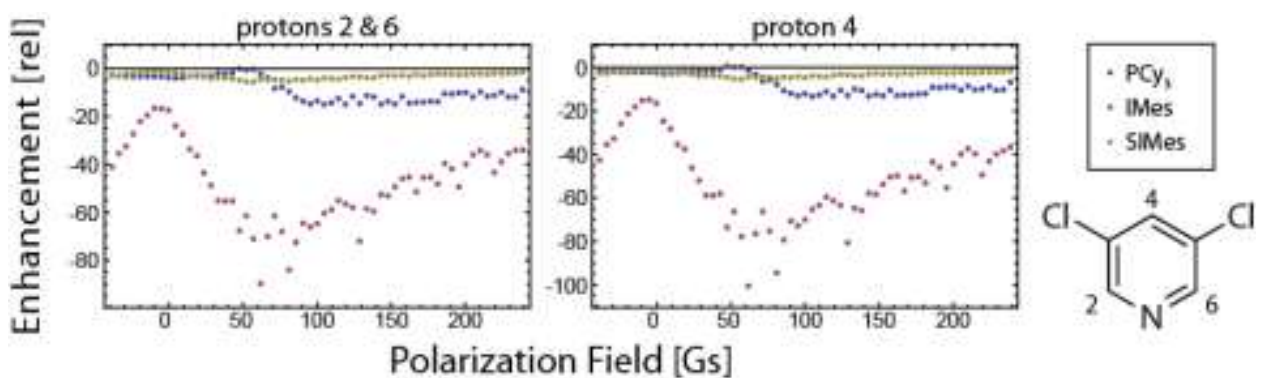

Figure 54: Polarization field dependence of signal enhancement for the protons in 3,5-dichloropyridine determined with various catalysts. The molecular structure of the substrate molecule is given on the right.

Comparison of enhancements for substrates exhibiting different $p K_{a}$ values

The results for the comparative examination of the 3-substituted pyridines given in Table 1 together with the applicable $\mathrm{pK}_{\mathrm{a}}$ values [126]. As can be seen, no clear correlation between the basicity and the achieved level of enhancement is discernable.

Crabtree's catalyst attained highest enhancements for the bromo derivative with a $\mathrm{pK}_{\mathrm{a}}$ of 2.80 to 2.85 , followed by the fluoro analog with a reported $\mathrm{pK}_{\mathrm{a}}$ of 2.79 to 3.10 . 3-Chloropyridine, with an almost identical $\mathrm{pK}_{\mathrm{a}}$ value, experienced, however, only about $50 \%$ of their enhancement levels. A slight increase of the $\mathrm{pK}_{\mathrm{a}}$ to the level of 3-iodopyridine also resulted in a reduction of enhancement, while a larger increase from there resulted again in higher levels of polarization for 3-methyl- and 3-methoxypyridine. Also, 3-hydroxypyridine with a $\mathrm{pK}_{\mathrm{a}}$ similar to 3-methoxypyridine, was only weakly enhanced, yet possibly owed to further effects caused by the tautomeric interconversion.

In the case of the IMes ligand, highest levels of polarization were achieved for 3-methoxyand 3-fluoropyridine, which exhibit strongly deviating basicity. The remaining halogen substituted pyridines (excluding 3,5-dichloropyridine) experience level of polarization reduced by approx. $35 \%$, yet of similar magnitude among them. Likewise, the methyl and hydroxy analogs experience only a slight reduction of enhancement, but also of comparable size.

For the SIMes ligand, reduction of the pyridine basicity by substitution with halogens proves detrimental to the achievable polarization. The further results slightly indicate an optimal 
range of electron density in the pyridine heterocycle of above that of the halogen derivatives and below the one for the examined methyl substituted compound, as highest enhancement was achieved for 3-hydroxypyridine, followed by 3-methoxypyridine, both of which possess $\mathrm{pK}_{\mathrm{a}}$ values in this range. Due to the low number of examined substrates in this pKa range, however, the basis for this postulation is extremely shaky.

Table 1: Maximum absolute enhancements achieved for various 3-substituted pyridines using different catalysts. ${ }^{1)}$ Experimentally determined values referenced in [126], except for ${ }^{2)}$, which was retrieved from [128]. ${ }^{3)}$ indicates the $\mathrm{pK}_{\mathrm{a}}$ for addition of a proton specifically to the nitrogen in the neutral form of 3-methoxypyridine [125] as compared to the compound value for the neutral and the zwitterionic form.

\begin{tabular}{c|c|cccc|cccc|cccc}
\multicolumn{10}{c|}{} & \multicolumn{10}{c|}{ Imes } \\
\hline substituent & $\mathbf{p K}_{\mathbf{a}}{ }^{\mathbf{1}}$ & H2 & H4 & H5 & H6 & H2 & H4 & H5 & H6 & H2 & H4 & H5 & H6 \\
\hline methyl & $5.67-5.75$ & 109 & 102 & 86 & 110 & 211 & 213 & 96 & 192 & 63 & 66 & 32 & 61 \\
methoxy & $4.78-4.90$ & 118 & 81 & 89 & 111 & 275 & 229 & 149 & 233 & 103 & 85 & 60 & 91 \\
hydroxy & $4.80-4.86 / 5.2^{3)}$ & 46 & 17 & 27 & 37 & 273 & 159 & 130 & 193 & 274 & 157 & 170 & 188 \\
flouro & $2.79-3.10$ & 141 & 134 & 80 & 131 & 265 & 261 & 158 & 231 & 15 & 14 & 9 & 13 \\
chloro & $2.81-2.98$ & 78 & 73 & 51 & 62 & 160 & 155 & 74 & 134 & 15 & 14 & 7 & 12 \\
bromo & $2.80-2.85$ & 151 & 143 & 93 & 141 & 181 & 172 & 84 & 149 & 23 & 22 & 10 & 18 \\
iodo & 3.25 & 82 & 78 & 58 & 69 & 173 & 171 & 70 & 147 & 25 & 23 & 10 & 21 \\
dichloro & $0.76^{2)}$ & 15 & 13 & - & 15 & 90 & 101 & - & 90 & 6 & 5 & - & 5 \\
\hline
\end{tabular}

Several more pyridine derivatives were examined in the same fashion as those above. In the meantime, however, the sample transfer parameters were optimized, which led to reduced transfer times and consequently to higher observed levels of polarization. Therefore, these substrates cannot be directly included in the basicity study, but are presented in the following:

\section{4-Iodopyridine}
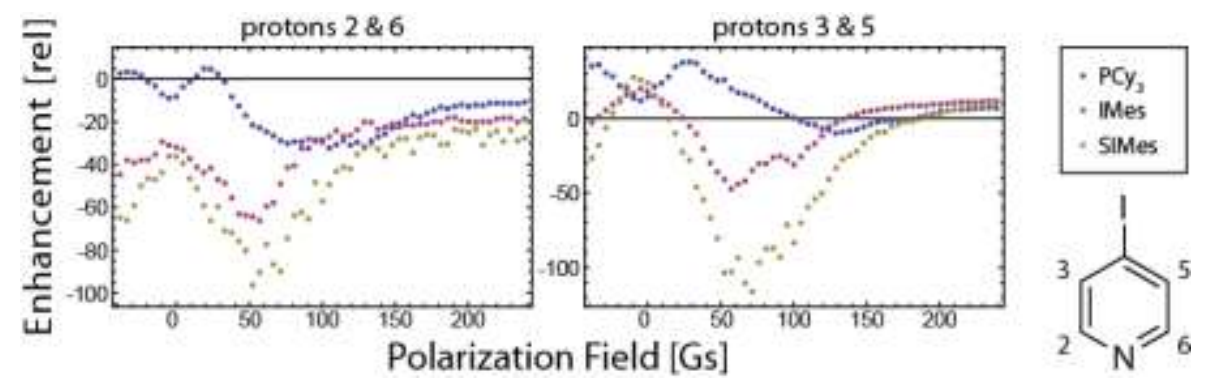

Figure 55: Polarization field dependence of signal enhancement for the protons in 4-iodopyridine determined with various catalysts. The molecular structure of the substrate molecule is given on the right.

Figure 55 displays the results for 4-iodopyridine, in which the IMes catalyst is surpassed by the SIMes ligand for highest enhancement of all substrate protons. With a $\mathrm{pK}_{\mathrm{a}}$ of 4.06 [129], the basicity of the substrate is in the range which is hinted to be suitable for an efficient hyperpolarization using the latter catalyst. The IMes complex still surpasses the $\mathrm{PC}_{3}$ catalyst by about a factor of 2 for $\mathrm{H} 2$ and $\mathrm{H} 6$, while the enhancement for the remaining protons is of comparable magnitude. 


\section{3,5-Dibromopyridine}
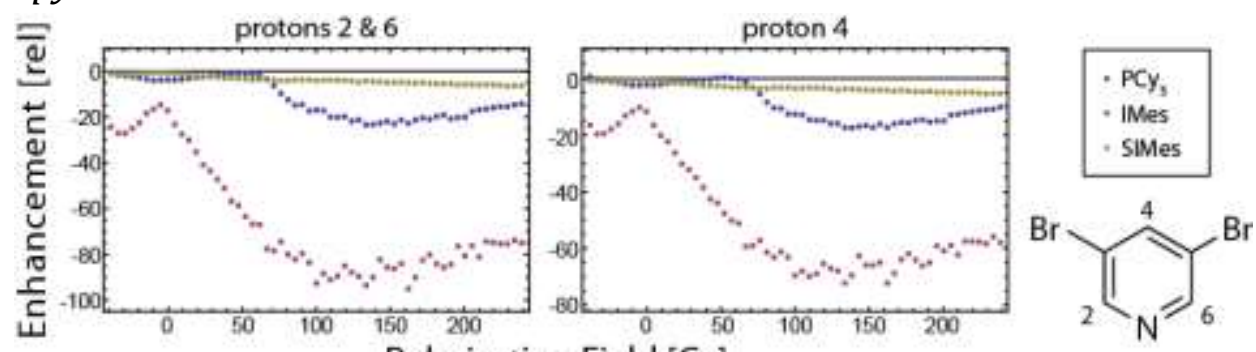

Polarization Field [Gs]

Figure 56: Polarization field dependence of signal enhancement for the protons in 3,5-dibromopyridine determined with various catalysts. The molecular structure of the substrate molecule is given on the right.

3,5-Dibromopyridine exhibits properties similar to those reported above for 3,5 -dichloropyridine. With a $\mathrm{pK}_{\mathrm{a}}$ value of 0.83 , the basicity is comparably low, and the binding affinity is accordingly presumed to be severely reduced, resulting in poor enhancement rates (Figure 56). The enhancement maximum at higher polarization fields is broadened for the $\mathrm{PCy}_{3}$ catalyst, similar to that reported for the dichloro analog. Furthermore, the maximum for the IMes ligand appears broadened and is displaced to higher fields.

\section{3,5-Dimethylpyridine}

In 3,5-dimethylpyridine, the electron density is raised by incorporation of two electron releasing substituents. Accordingly, the basicity is even higher than of the previously examined 3-methylpyridine, with a $\mathrm{pK}_{\mathrm{a}}$ of 6.14 [130]. This proves beneficial for Crabtree's catalyst, which surpasses the enhancements achieved by the IMes catalyst for all substrate protons (Figure 57). For this analyte, the maximum enhancement for the ring protons achieved by the SIMes complex is shifted to higher field. Interestingly, the maximum for the methyl protons remains unchanged.

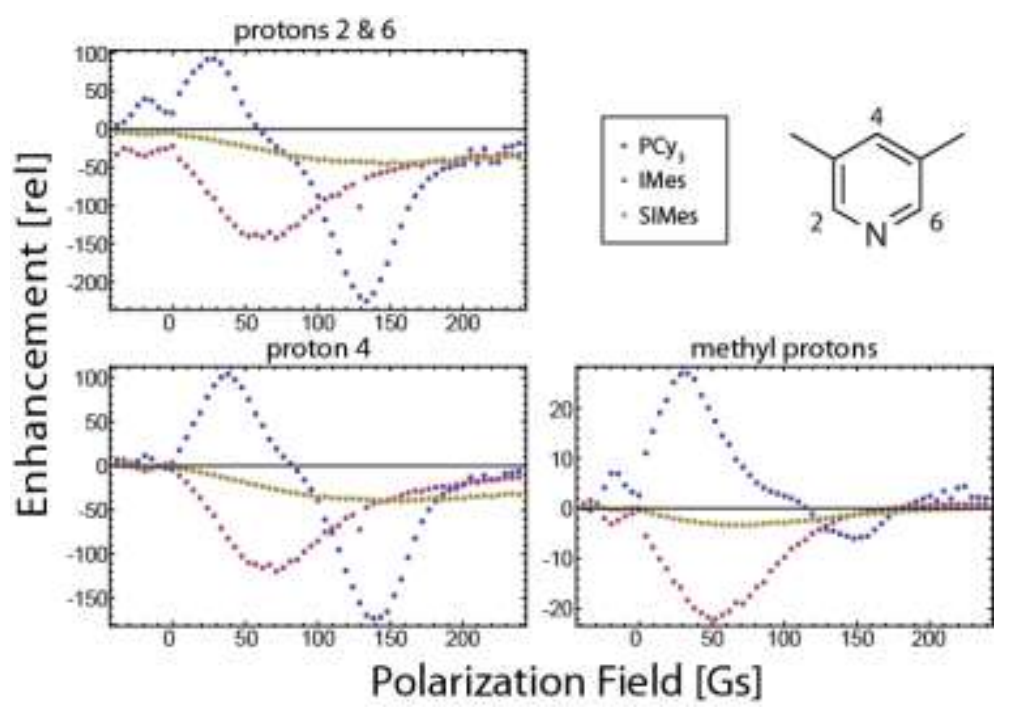

Figure 57: Polarization field dependence of signal enhancement for the protons in 3,5-dimethylpyridine determined with various catalysts. The molecular structure of the substrate molecule is given at the top right. 


\section{3,4-Dimethylpyridine}

The difficulty in correlating the $\mathrm{pK}_{\mathrm{a}}$ values and the attained enhancement is further illustrated by the results for 3,4-dimethylpyridine (Figure 58). This substrate exhibits a pKa value of 6.48 [130], close to the one reported for the former substrate. However, the polarization levels achieved by Crabtree's catalyst are significantly reduced compared to 3,5-dimethylpyridine and show clearly inferior enhancement values compared to the IMes complex. Both NHC catalysts achieve enhancements of a larger magnitude compared to the former substrate, with even the SIMes ligand surpassing the $\mathrm{PCy}_{3}$ complex for all substrate protons by a wide margin.
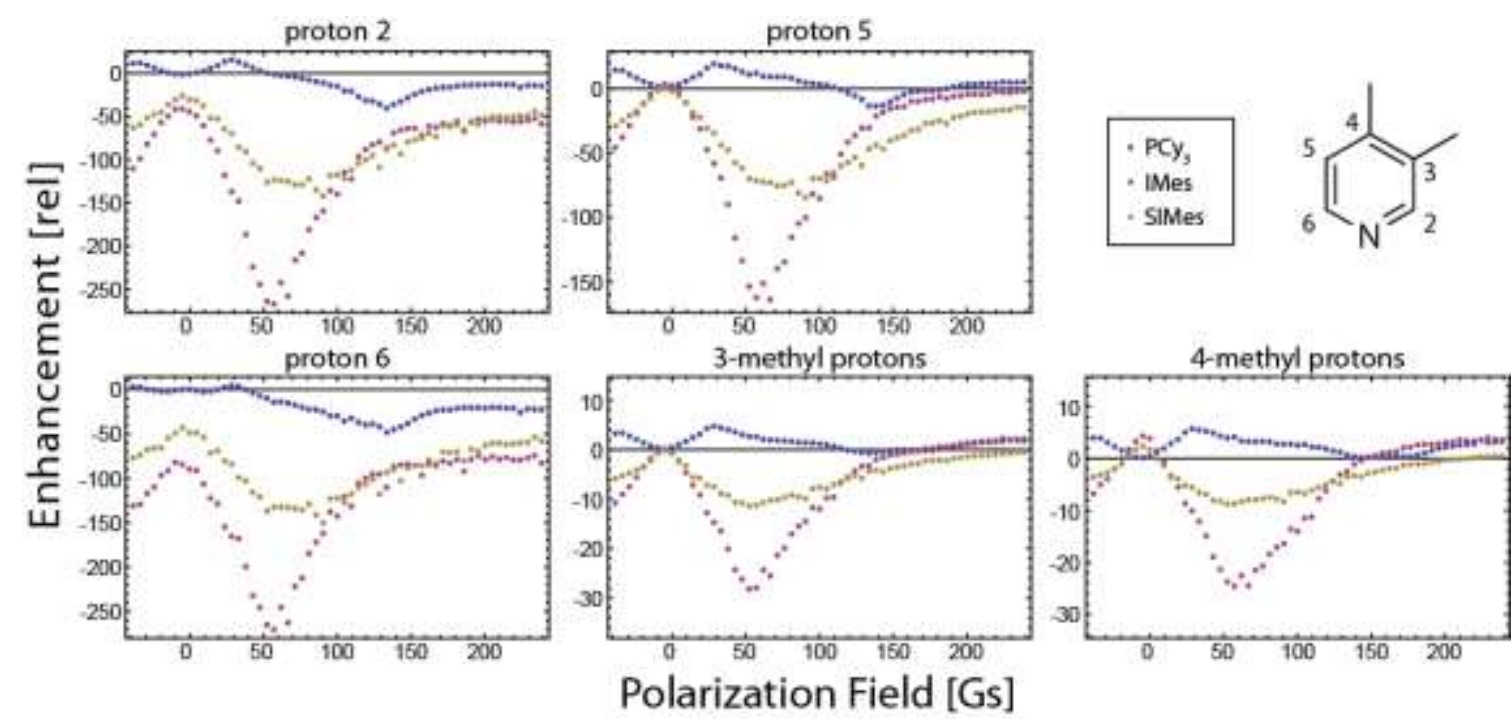

Figure 58: Polarization field dependence of signal enhancement for the protons in 3,4-dimethylpyridine determined with various catalysts. The molecular structure of the substrate molecule is given at the top right.

\subsubsection{Discussion of catalyst performances}

In summary, it can be concluded that a direct correlation between the substrate basicity, indicated by their $\mathrm{pK}_{\mathrm{a}}$ values, and the achieved level of enhancement cannot be postulated from the obtained results and further factors that have an influence on the ligand exchange rate and $J$-couplings within the active complex might have to be considered.

For a range of various substrates, the IMes ligand proved to be the superior transfer agent for $\mathrm{pH}_{2}$-derived hyperpolarization, as was initially reported [34]. For selected substrates, however, the competing $\mathrm{PCy}_{3}-$ and SIMes complexes proved to be equivalent or even superior. Furthermore, the polarization field strength at which the highest levels of enhancement were generated differed between Crabtree's catalyst and the NHC complexes, indicating the use of the former in cases of inevitable elevated field strengths. Additionally, the NHC catalysts almost exclusively generate emissive signal patterns, while the $\mathrm{PCy}_{3}$ complex for most substrates produces both, emissive and absorptive lines depending on the selected polarization field, both of which was described by Pravdivtsev et al. in theoretical studies [37, 80]. Lastly, using the $\mathrm{PC}_{3}$ catalyst, different protons within a substrate can experience signal enhancement of strongly deviating magnitude at identical polarization fields. This is best illustrated by considering the results for various pyridine derivatives, e.g., 
3-methylpyrazole in Figure 46. For this substrate, $\mathrm{H} 2, \mathrm{H} 4$ and $\mathrm{H} 6$ all experience a zero transition in their enhancement factors in the region between 50 and $60 \mathrm{Gs}$, while $\mathrm{H} 5$ in this range exhibits almost its highest level of polarization. Conversely, for H5, almost no enhancement can be detected for fields of 135 to $150 \mathrm{Gs}$, at which the remaining ring protons clearly exhibit emissive maxima. Within limits, it is therefore possible through deliberate polarization field selection to exclude certain signals while highly enhancing others using the $\mathrm{PCy}_{3}$ catalyst. In short, the IMes catalyst exhibits the highest potential for tremendous signal enhancement, while Crabtree's catalyst offers a certain potential for precision tuning.

\subsubsection{Discussion of Experimental Setup 2}

The new setup was successfully taken into operation and produced highly enhanced spectra for a variety of substrates and catalysts. Owed to the superior resolution, which, for the hyperpolarization spectra, was on par with the finely adjusted reference spectra, unimpeded signal examination was possible. Full automation of the experimental procedure served to eliminate all timing variances between individual hyperpolarization experiments, contributing to an increased comparability of measurements at different fields. Furthermore, the time necessary to conduct an entire measurement series was significantly reduced while considerably alleviating the physical stress on both equipment and personnel.

Despite these significant improvements to the experimental procedure, a number of substantial flaws are apparent for this setup:

The determination of the perfectly timed transfer parameters, which is crucial for conducting the hyperpolarization experiments, proved to be tedious. When parameters were determined for the dummy flow cell, they were not directly applicable to the actual flow injection probe and had to be painstakingly adjusted, which often resulted in unnecessarily elongated transfer times. Furthermore, when a set of transfer parameters gave satisfactory results, the reproducibility of the transfers left a great deal to be desired. Using the dummy flow cell, it was readily observable that the achieved liquid levels at the end of the transfer process fluctuated even for experiments executed in direct succession. Visual observation often revealed a default rate of up to $50 \%$ when examining the transfer efficiency. In order to account for these severe fluctuations, the transfer parameters had to be adjusted to rigorously overfill the flow cell to ensure a minimum of flawed or unsuccessful experiments. This, again, resulted in extended transfer times, leading to a decrease in observable hyperpolarization.

However, when the risk of overfilling the cavity and losing some of the sample volume in each transfer was accepted, and the transfer parameters were adjusted to rapidly and reliably fill the flow probe at the highest speed possible, this experimental setup was suitable to achieve outstanding levels of hyperpolarization, as the exemplary spectrum in Figure 59 illustrates. Therein, $\mathrm{H} 4$ experienced a more than thousandfold enhancement at a field of $52 \mathrm{Gs}$, with $\mathrm{H} 2$ and $\mathrm{H} 6$ closely behind. With a setting like this, however, the loss of 
sample volume increased significantly, so that measurement series spanning the modulation field range from -145 to $145 \mathrm{Gs}$ in $5 \mathrm{Gs}$ increments could not be completed successfully.

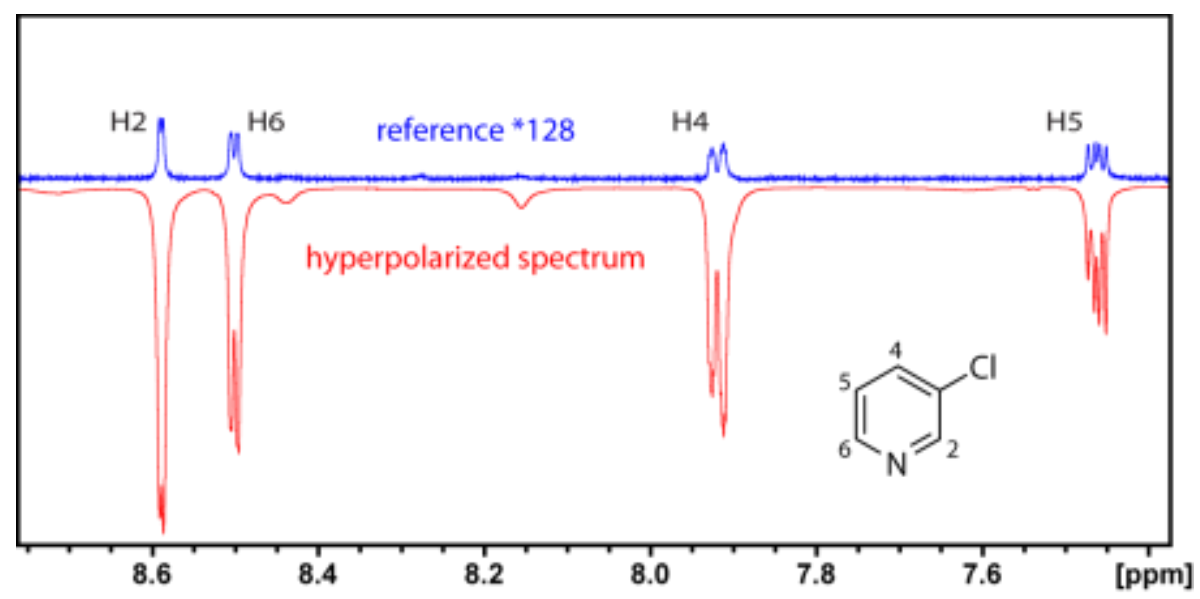

Figure 59: Exemplary spectrum overlay of the aromatic region of 3-chloropyridine. The hyperpolarized spectrum was recorded at a polarizer setting of $-50 \mathrm{Gs}$, resulting in an effective polarization field of $52 \mathrm{Gs}$. The reference spectrum is enhanced by a factor of 128 . Additional resonances in the hyperpolarized spectra originate from hyperpolarized substrate molecules coordinated to the polarization transfer catalyst.

The aforementioned loss of sample volume also constitutes a drawback of this setup and occurs in three different fashions. During the polarization step, for which the $\mathrm{H}_{2}$ gas enriched in its para isomer is bubbled through the sample solution, parts of the liquid are propelled to the top of the mixing chamber due to vigorous foaming. From there, droplets can be forced through an exhaust tubing, leading to a loss of solvent and of the therein dissolved molecules. In a similar manner, small fractions of the sample are lost when it is incompletely returned from the flow injection probe and remains as small droplets in the tubing system. This is especially the case when the probe cavity is overfilled, which was done in order to account for the transfer fluctuations and was accepted for the sake of reliability. As all sample components are expelled together, this results only in the reduction of sample volume.

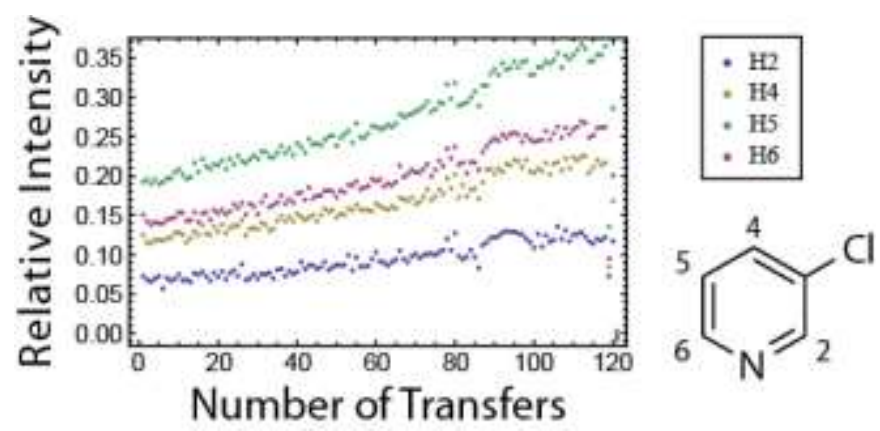

Figure 60: Signal intensities of the protons in 3-chloropyridine in dependence of the number of executed sample transfers. Due to the limited time in the spectrometer, the Boltzmann polarization is not reached, resulting in relative signal intensities $<1$. Differences in the signal intensities between individual protons stem from the individual relaxation times.

Evaporation of the deuterated solvent denotes the third way of sample volume loss. Due to the foaming during the polarization step and the perturbance during the return transfer, the 
liquid phase exhibits a large surface area to the gas phase, mediating a high level of saturation with the gaseous solvent. The gas phase is exchanged between the individual experiments, resulting in a slow but constant loss of solvent, leaving behind the substrate molecules and the metal complexes. This leads to an effective concentration of the sample, observable in Figure 60.
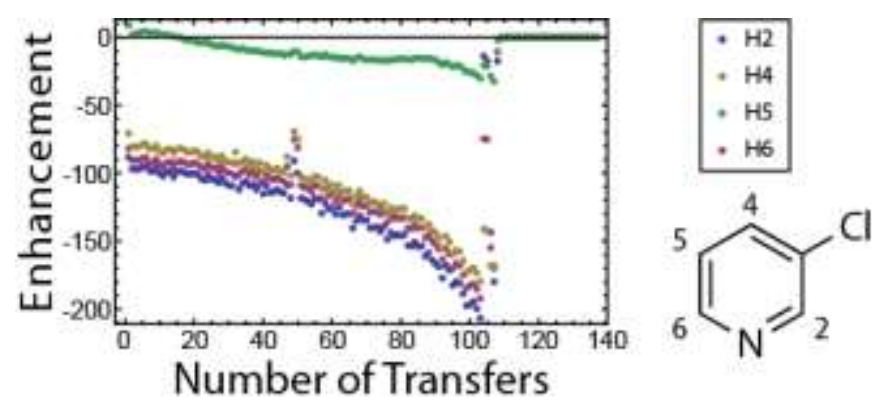

Figure 61: Level of enhancement achieved for the protons in 3-chloropyridine at a field of 5 Gs in dependence of the number of experiments performed. The IMes polarization transfer catalyst was used to facilitate the hyperpolarization.

In addition to the increased concentration, the change in sample volume has an additional effect on the spectra. Due to the field gradient along the length of the mixing chamber, the field strengths contributing to the observed level of hyperpolarization is directly dependent on the fill level over which the sample is distributed. With lower sample volumes, the spread decreases, resulting in a narrower distribution of magnetic fields and consequentially in a change of the observed enhancement. This is illustrated in Figure 61, which displays the achieved enhancements for 3-chloropyridine using the IMes polarization transfer catalyst at an effective field of $5 \mathrm{Gs}$ (measured at a depth of $7 \mathrm{~cm}$ into the coil cavity) in dependence of the number of performed experiments. Not only does the enhancement level for protons 2 , 4 and 6 increase in a seemingly exponential fashion, which was expected due to the change in concentration, but the $\mathrm{H} 5$ resonance also experiences a change from absorptive to emissive signal after a number of transfers, which can only be explained by a change in the contributing magnetic fields, at which the polarization transfer was achieved.

Figure 62 further illustrates this effect. Therein, the enhancements achieved for $\mathrm{H} 2$ in 3-chloropyridine are given in dependence of the direction in which the polarization field was modulated. In the left profile, the experimental series was initiated from the low field side and the effective field strength was consecutively increased in steps of $5 \mathrm{Gs}$. For the right profile, the initial experiment was performed at the highest field and the polarization field stepwise decreased. The two polarization field patterns exhibit polarization fields at which maximum enhancement was achieved, which differ by approx. $10 \mathrm{Gs}$, owed, presumably, to the difference in sample volume as a result of the total number of experiments performed with the loaded sample. 


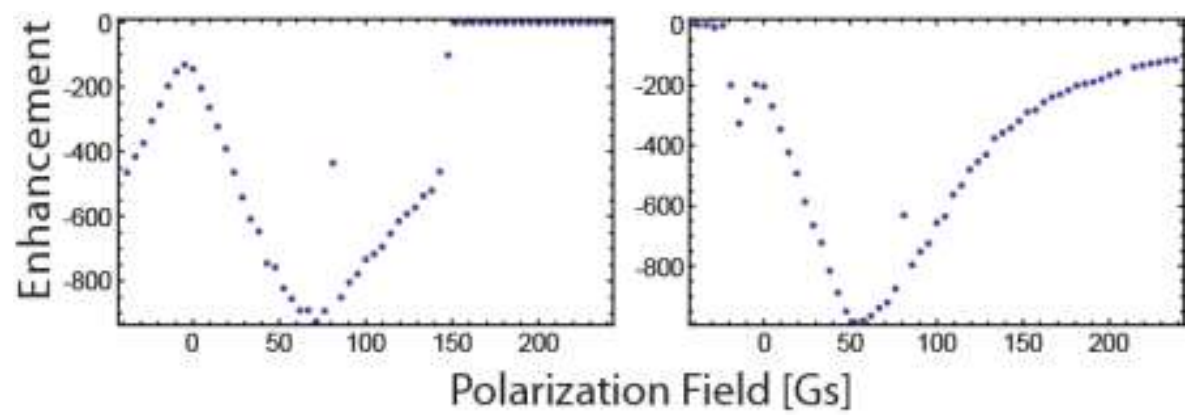

Figure 62: Polarization field dependence for $\mathrm{H} 2$ in 3-chloropyridine using the IMes polarization transfer catalyst. Both field dependence profiles were recorded in direct succession using identical sample compositions and parameters with the exception of the direction in which the polarization field was modulated.

The control software of the Polariser was later updated by the manufacturer to account for the sample loss via a so called "top up" option. In order to utilize it, the amount of sample loss per transfer was determined by comparison of the liquid level before and after the experiments and subsequent division of the lost volume by the number of conducted experiments. The determined sample loss volume was then added from the connected sample vial into the mixing chamber after each experiment. The liquid level could be held relatively constant over the course of an experimental series, yet the sample concentration gradually increased, owed to the evaporation of solvent as a contributor to the sample loss.
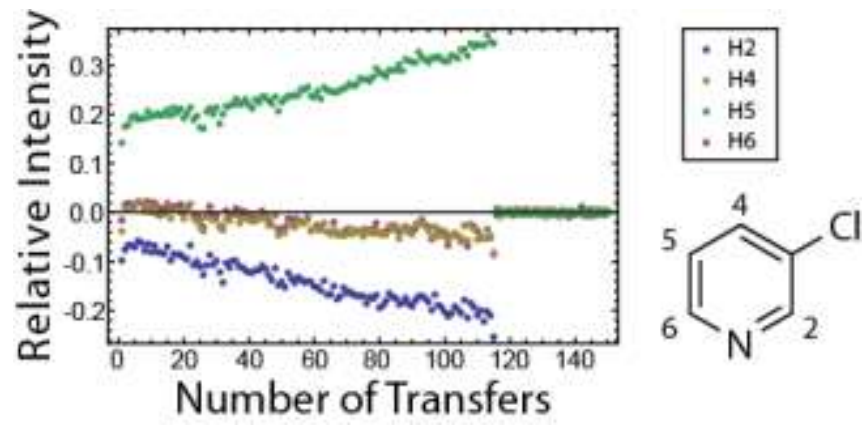

Figure 63: Signal intensity for the protons in 3-chloropyridine in dependence of the number of performed sample transfers. The observed transfer of hyperpolarization is achieved by residual catalysts within the tubing system that withstood an extended washing operation.

A minor flaw of the Bruker Para-Hydrogen Polariser setup can be seen in the difficulty of cleaning the entire flow path, including syringe, mixing chamber and flow injection probe, connected via the small diameter tubing. All of these parts possess joints and crevices, in which molecules can deposit and from which they can only slowly be cleared. Even after several hours of constant flushing using pure ethanol, a sample of 3-chloropyridine in $\mathrm{CD}_{3} \mathrm{OD}$ still showed emissive signals after application of $\mathrm{pH}_{2}$ (Figure 63), indicating the presence of residual catalytic complexes in the flow path. The extent of polarization achieved is, fortunately, relatively small and can be considered negligible compared to that achieved by the actual employed catalyst.

The main flaw this experimental setup exhibited was constituted, however, by the relatively large field gradient over the sample volume and the associated uncertainty about the 
effective polarization field the sample experienced. As described above, the field divergence over three characteristic points along the height of the mixing chamber is in the range of 27 Gs. For highest polarization fields, this spread is reduced to $14 \mathrm{Gs}$, corresponding to a satisfactory percentage variation of approx. $6 \%$. For the other end of the polarization field spectrum, however, the difference between highest and lowest field at these points amounts to $34.5 \mathrm{Gs}$, oftentimes surpassing the targeted field strength by a multiple (Figure 64).

Unfortunately, the low field range exhibits a higher information density considering the maxima location of the NHC catalysts. With such a broad range of fields contributing to the polarization transfer, smaller variances cannot be resolved, and the entire profile is smoothened. Furthermore, the intensities at the enhancement maxima are reduced, as these fields cannot be individually examined, but experience a contribution of fields at which the polarization transfer is less efficient.

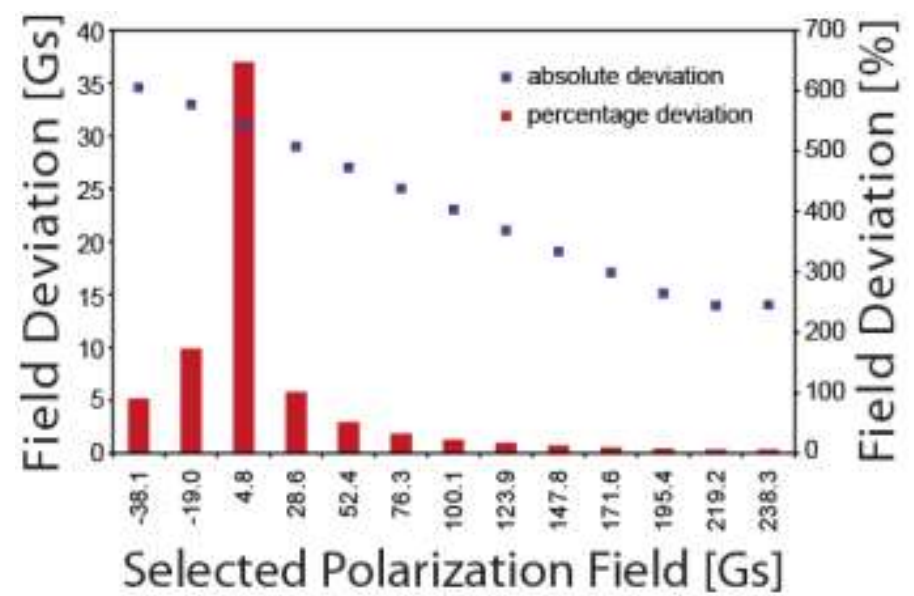

Figure 64: Deviations from the selected polarization field over the effective sample volume in the mixing chamber. Percentage deviations are referenced to the appointed field at the lowest of three characteristic points of the sample volume. Absolute field deviations are given as the difference between highest and lowest field at these three locations.

In order to reduce the size of the field gradient over the sample volume, the mixing chamber has to be moved further away from the spectrometer, to where the stray field profile exhibits a shallower slope. As the mixing chamber has to be positioned directly below the NMR magnet, this can only be achieved by moving the chamber to a lower position. For this setup, the chamber was unfortunately already positioned at the lowest possible setting, forfeiting the possibility of an adjustment. Moving to a spectrometer with a weaker $\mathrm{B}_{0}$ field and consequently a weaker stray field poses a feasible, yet expensive alternative, as the employed flow injection probe is incompatible with smaller sized NMR magnets and an additional probe would have to be acquired.

As a technical solution, employment of a shimming system may be feasible, that would homogenize the field in the polarization chamber. Unfortunately, development of the Para- 
Hydrogen Polariser has been discontinued by the manufacturer, such that this extensive modification of the technical device would have to be done by additional experts in house. 


\subsection{Chemical Synthesis}

A prerequisite for the hyperpolarization of a substrate using the SABRE technique is the presence of a donor atom in the molecular structure. The molecule has to be able to reversibly bind to the polarization transfer catalyst using its free electron pair and must do so at a considerable turnover rate in order to allow for the polarization of a large number of individual molecules. Pyridine, pyrazole and their derivatives, which were studied extensively in this work, exhibit this property and were therefore, as was shown, highly susceptible to the polarization technique. Their chemical structure can often be found as a substructure in biologically active compounds, creating the opportunity for in vivo studies of distribution and metabolization using the SABRE approach.

The employment of heavy metal containing catalysts that facilitate the polarization transfer from the parahydrogen nuclei to the substrate of interest constitutes an obstacle to the unmodified application to living organisms. To prevent a possible toxic effect [131] to the studied subject, it must be ensured that the sample solution can be separated from the catalyst before its transfer into the organism. This can be achieved by modification of the critical ligand molecule in a way that allows for permanent binding to macroscopic particles, e.g., polystyrene beads, which in turn can be easily removed from the liquid phase by means of filtration.

\subsubsection{Synthesis of a solid-phase bound SABRE catalyst}

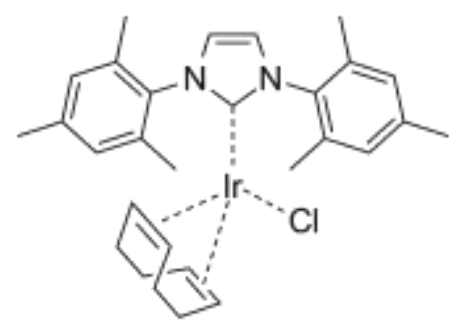

Figure 65: Structure of $[\operatorname{IrCl}(\mathrm{COD})(\mathrm{IMes})]$ containing the IMes ligand. The complex reacts with suitable substrates and $\mathrm{pH}_{2}$ to generate an active polarization transfer catalyst.

1,3-Bis(2,4,6-trimethylphenyl)imidazol-2-ylidene (IMes) is the critical ligand in $[\operatorname{Ir}(\mathrm{COD})(\mathrm{IMes}) \mathrm{Cl}]$ (Figure 65), which is the precursor of the SABRE catalyst showing the highest potency to date [45]. The para methyl substituents at the phenyl rings were considered promising connection points for a modification of the ligand without altering the steric effects and the electronic parameters in the imidazole-2-ylidene heterocycle. 


\subsubsection{Definition of initial target molecule}

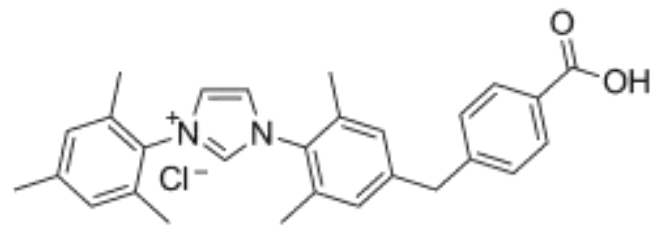

1

Figure 66: Compound 1 was appointed as the initial target molecule for the synthesis of a solid-phase bound ligand to be used in SABRE hyperpolarization experiments.

Compound 1 (Figure 66) was selected as the target molecule for the synthesis. The electronic composition of the original molecule is largely conserved and with the carboxyl function, a connection site for attachment to a solid phase is introduced. To create a donor atom, the methine proton between the two nitrogen atoms is abstracted, transforming the molecule into a carbene.

The developed synthesis strategy for compound $\mathbf{1}$ is shown in Scheme 6.
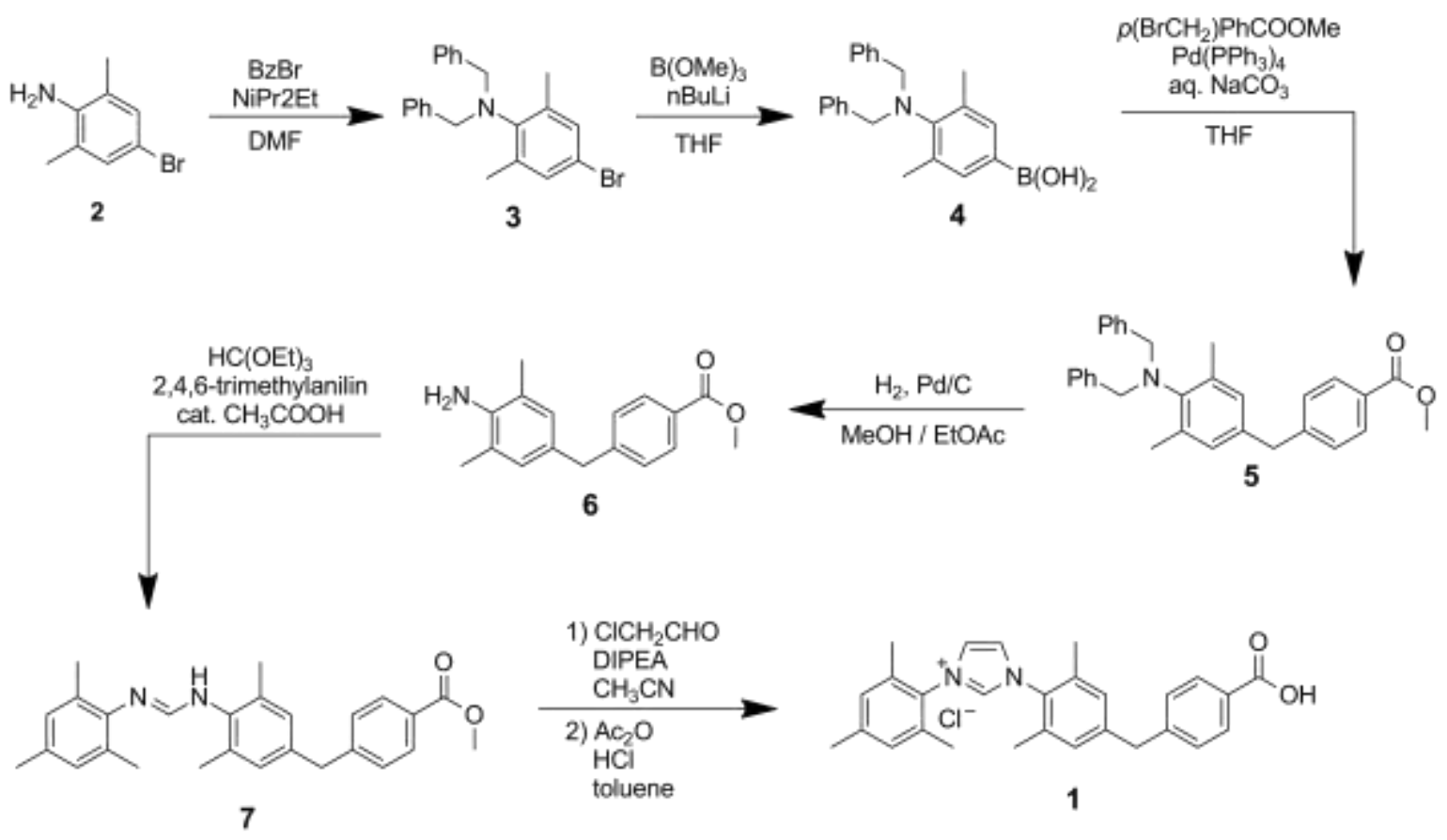

Scheme 6: Synthesis strategy developed for compound 1. 


\subsubsection{Synthesis of imidazolium chloride 1}

Starting from aniline 2, methyl ester $\mathbf{5}$ was successfully synthesized in 3 steps (Scheme 7).

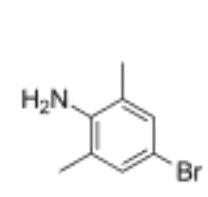

2

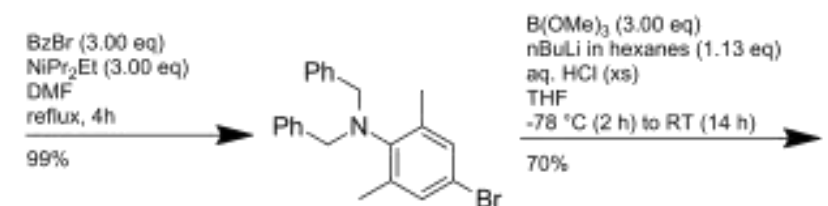

3

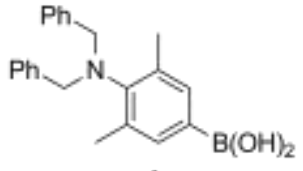

4

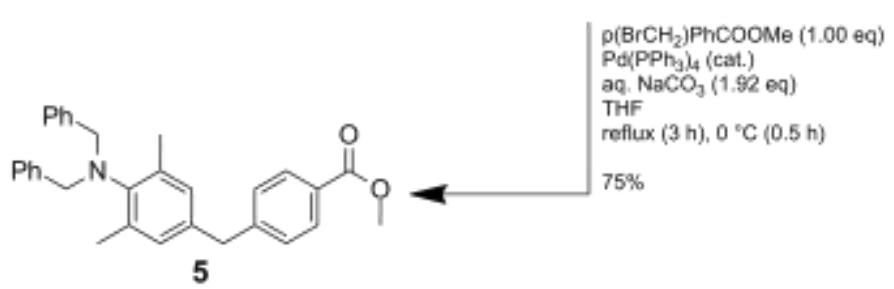

Scheme 7: The reaction steps of aniline protection, boronic acid formation and Pd-catalyzed coupling reaction to synthetize compound $\mathbf{5}$ in three steps, starting from 4-bromo-2,6-dimethylaniline.

The amine function in aniline $\mathbf{2}$ was protected by treatment with $\mathrm{N}, \mathrm{N}$-diisopropylethylamine and benzyl bromide and the resulting tertiary amine $\mathbf{3}$ was obtained in quantitative yield. Subsequently, the aromatic bromine of $\mathbf{3}$ was replaced by a boronic acid group, which was achieved by treatment with $n$-butyllithium and trimethyl borate, followed by hydrolysis using aqueous hydrochloric acid. Boronic acid 4 was received in good yield of $70 \%$. In the next step, a palladium catalyzed cross-coupling between $\mathbf{4}$ and methyl 4-bromobenzoate was successfully performed to give benzhydryl 5 in satisfactory $75 \%$ yield.

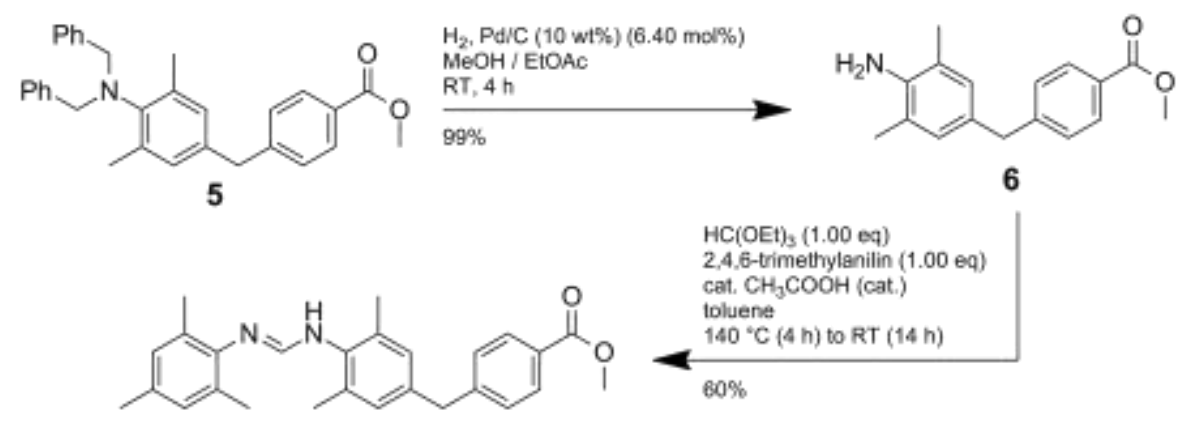

7

Scheme 8: Cleavage of the protections groups and coupling reaction with 2,4,6-trimethylaniline to give formamidine $\mathbf{7}$ in two steps.

In two further steps, formamidine 7 was obtained (Scheme 8). First, the benzyl protection groups in $\mathbf{5}$ were successfully cleaved using hydrogen gas and palladium on carbon as a catalyst, giving primary amine 6 in quantitative yield. In the following step, the received amine was coupled to 2,4,6-trimethylaniline via an acid-catalyzed reaction with triethyl orthoformate. The resulting formamidine 7 was isolated in a yield of $60 \%$.

In the next step, diisopropylamine and chloroacetaldehyde were used to facilitate a ringclosing reaction to create a hydroxy-substituted imidazoline heterocycle. As formamidine 7 exists in two tautomeric structures, the hydroxy function can be located at both of the newly 
introduced carbons. This reaction was planned to be immediately followed by an elimination reaction using acetic anhydride and hydrochloric acid in order to form the $\mathrm{C}$-C double bond in the imidazole moiety of $\mathbf{1}$. Formation of imidazolinium chloride $\mathbf{8}$ was verified by reaction control via mass spectrometry, yet dehydration to form 1 in the subsequent reaction step was not observed under the given conditions. The two-step reaction was repeated several times in order to facilitate the elimination reaction to produce imidazole $\mathbf{1}$. In addition to modifications of reactant concentrations and reaction conditions, a second elimination protocol using orthophosphoric acid was tested, yet compound $\mathbf{1}$ was not isolated (Scheme 9).

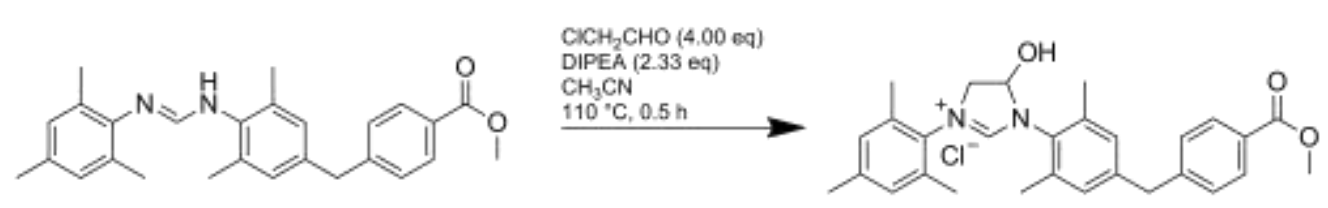

7

8

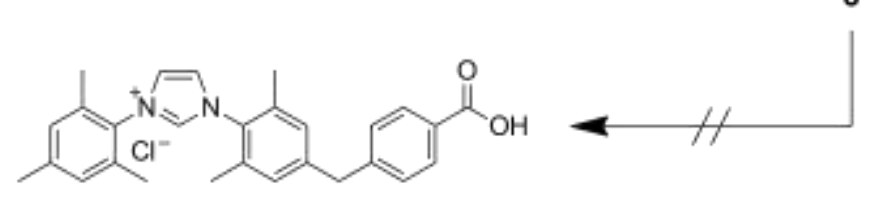

1

Scheme 9: The ring-closing reaction giving imidazolinium chloride 8 and the subsequent unsuccessful elimination reaction to reach target molecule 1.

\subsubsection{Change of synthesis target}

Due to the failed attempt in producing imidazolium chloride $\mathbf{1}$, compounds $\mathbf{9}$ and $\mathbf{1 0}$ (Figure 67) were appointed as new target molecules to be attached to a solid phase consisting of silica gel via their triethoxysilyl functions. The target molecules show a deviation from the IMes ligand, as they feature an imidazoline instead of an imidazole hetero cycle. This is accompanied by a change in the electronic composition, which has a strong impact on the functionality of catalysts formed using this ligand. The SIMes ligand, which also relies on the imidazoline ring, has, however, been shown to be an operational polarization transfer catalyst earlier in this work.

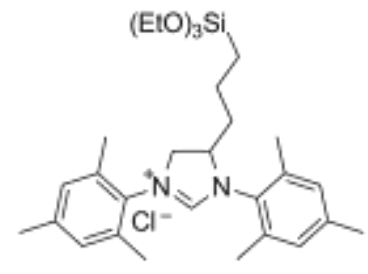

9

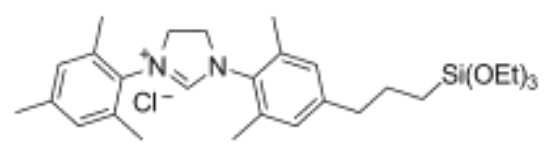

10

Figure 67: Compounds 9 and 10 were appointed as new synthesis targets.

Originated from different starting molecules, compounds 11 and 12 (Figure 68) were synthesized and isolated according to published synthesis protocols [132] in 2 and 7 steps, respectively. These molecules are the direct precursors of the target compounds $\mathbf{9}$ and $\mathbf{1 0}$ 
and were treated in a two-step reaction first with trichlorosilane and a palladium-based catalyst, then with ethanol and triethylamine to achieve the conversions into the triethoxysilanes.

Due to the products' susceptibility to hydrolysis and the absence of a dry box, the target molecules could not be isolated, as the column chromatography necessary for purification could not be performed under sufficiently water-free conditions.

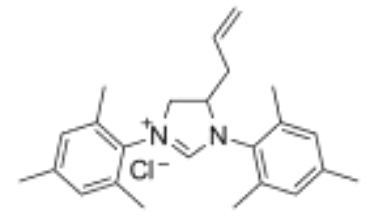

11

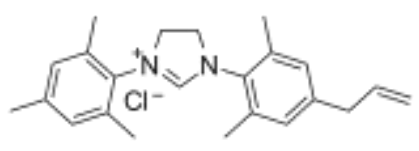

12

Figure 68: Compounds 11 and 12 are the respective isolated precursors of target molecules $\mathbf{9}$ and 10.

\subsubsection{Modified polymer beads}

Consequentially, the synthesis target and route were once more adjusted as depicted in Scheme 10. In the updated approach, an NHC ligand precursor was to be created directly at solid-phase particles by modification of functionalized polystyrene beads. Subsequently, the carbene was to be generated in situ and attached to the Ir center via a silver alkoxide.

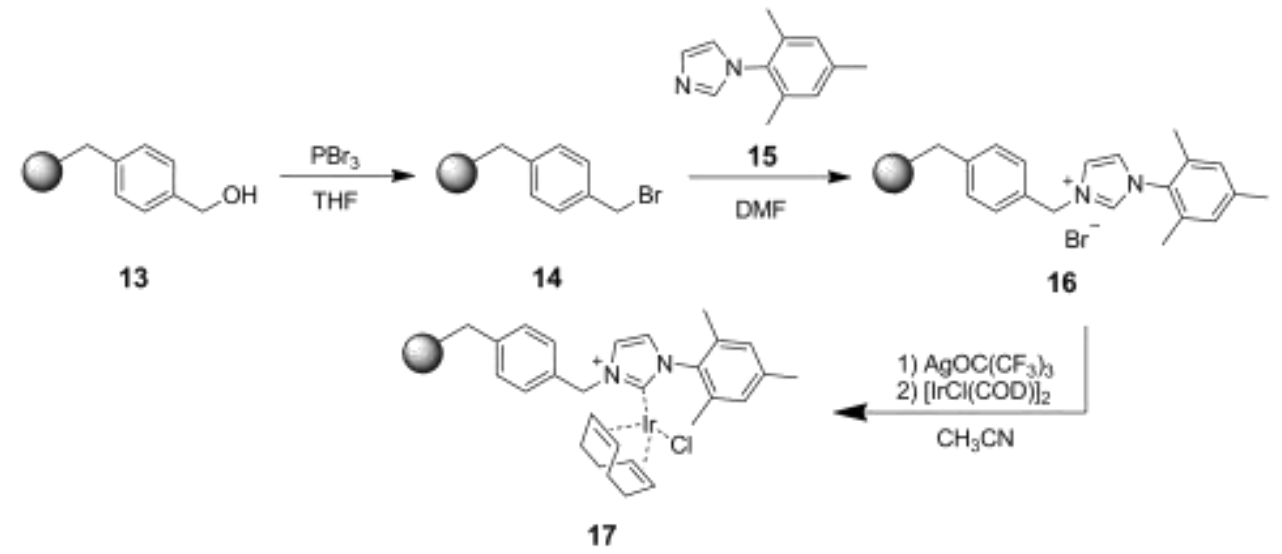

Scheme 10: Intended reaction route for the generation of an NHC ligand by modification of functionalized polymer beads and the subsequent creation of a solid-phase bound SABRE precatalyst complex. The polymer beads represented by the grey spheres consist of cross-linked polystyrene.

Treatment of functionalized polymer 13 with phosphorus tribromide yielded the benzyl bromide variant 14 . Polymer 14 was then treated with mesitylimidazole 15 , which had been prepared according to ref. [133], to give solid-phase bound imidazolinium bromide 16 as identified by the characteristic aromatic methyl resonances of the mesityl group in ${ }^{1} \mathrm{H} N M R$ (Figure 69). For the following synthesis step, however, the synthesis for the silver alkoxide reagent $\mathrm{AgOC}\left(\mathrm{CF}_{3}\right)_{3}$ could not be reproduced according to the reported synthesis protocol [134], as no chemical modification of the precursor molecule was observed in ${ }^{13} \mathrm{C}$ and ${ }^{19} \mathrm{~F}$ NMR spectra. An attempt at the preparation of complex 17 was nonetheless undertaken, yet the obtained product showed no activity in SABRE polarization transfer experiments. 


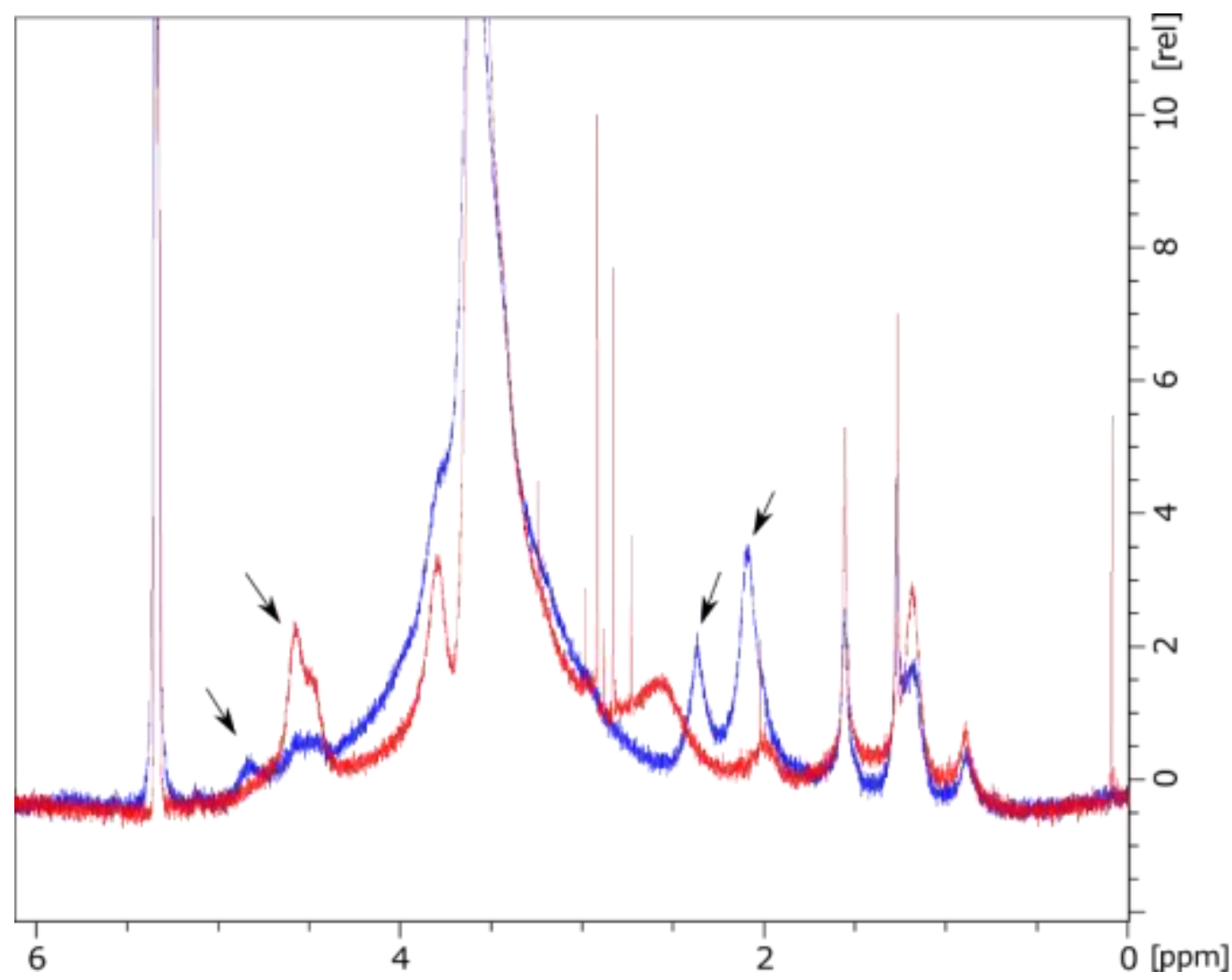

Figure 69: ${ }^{1} \mathrm{H}$ NMR spectra section of solid-phase bound benzyl bromide 14 (red) and imidazolinium bromide 16 (blue). Formation of 16 is clearly indicated by emergence of the indicated methyl resonances between 2.5 and $2.0 \mathrm{ppm}$ and the methine resonance at $4.8 \mathrm{ppm}$, while the intensity of the methine resonance at $4.5 \mathrm{ppm}$ is significantly reduced.

\subsubsection{Outlook}

Towards the very end of this study, compounds 18 (attached to cross-linked polystyrene beads with poly (ethylene glycol) functionalities, TentaGel ${ }^{\circledR}$ ) and 19 (attached to poly (ethylene glycol) beads, ChemMatrix ${ }^{\circledR}$ ) were provided by a fellow researcher and were used to form Iridium complexes $\mathbf{2 0}$ and $\mathbf{2 1}$ according to Scheme 11.
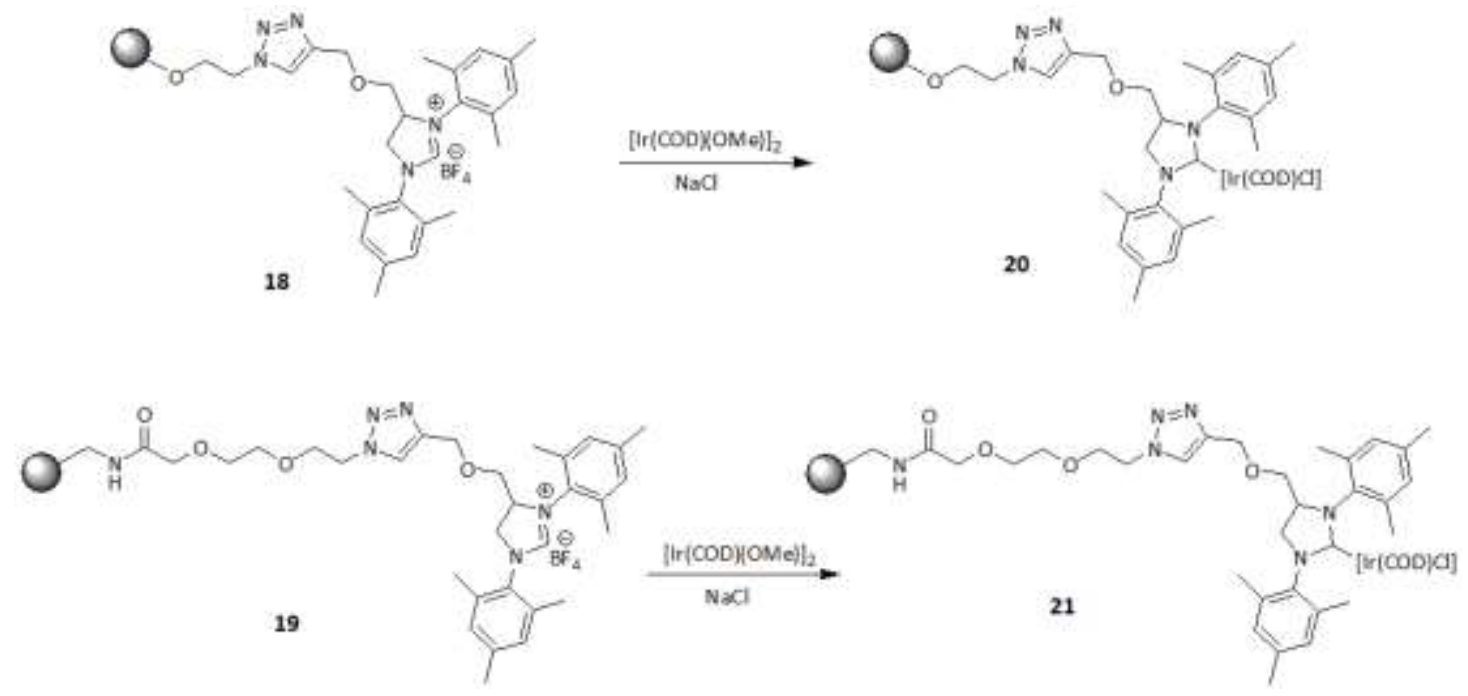

Scheme 11: Polymer-bound ligands 18 and 19 and their corresponding iridium complexes 20 and 21. 
Both complexes showed catalytical activity to facilitate the hydrogenation of a double bond to give a single bond under a hydrogen atmosphere, as shown Figure 70. Furthermore, SABRE experiments were conducted with a modified Polariser setup, in which the functionalized polymer beads were manually introduced into the polarization chamber. Furthermore, a syringe filter was added into the tubing system between the polarization chamber and the flow probe to separate the solution from the catalyst and to prevent the solid particles from reaching the flow cell within the probe head. Both catalysts showed polarization transfer activity in these experiments, as exemplified in Figure 71, where pyridine was used as the analyte in combination with complex $\mathbf{2 0}$ and a seven-fold enhancement was accomplished. While, however, both catalysts showed signs of leaching in these experiments, these results mark a promising starting point for future research into SABRE experiments with solid-phase bound polarization transfer catalysts.

As the results in this outlook section are preliminary, they have not been thoroughly quantified and do not appear in the experimental section.

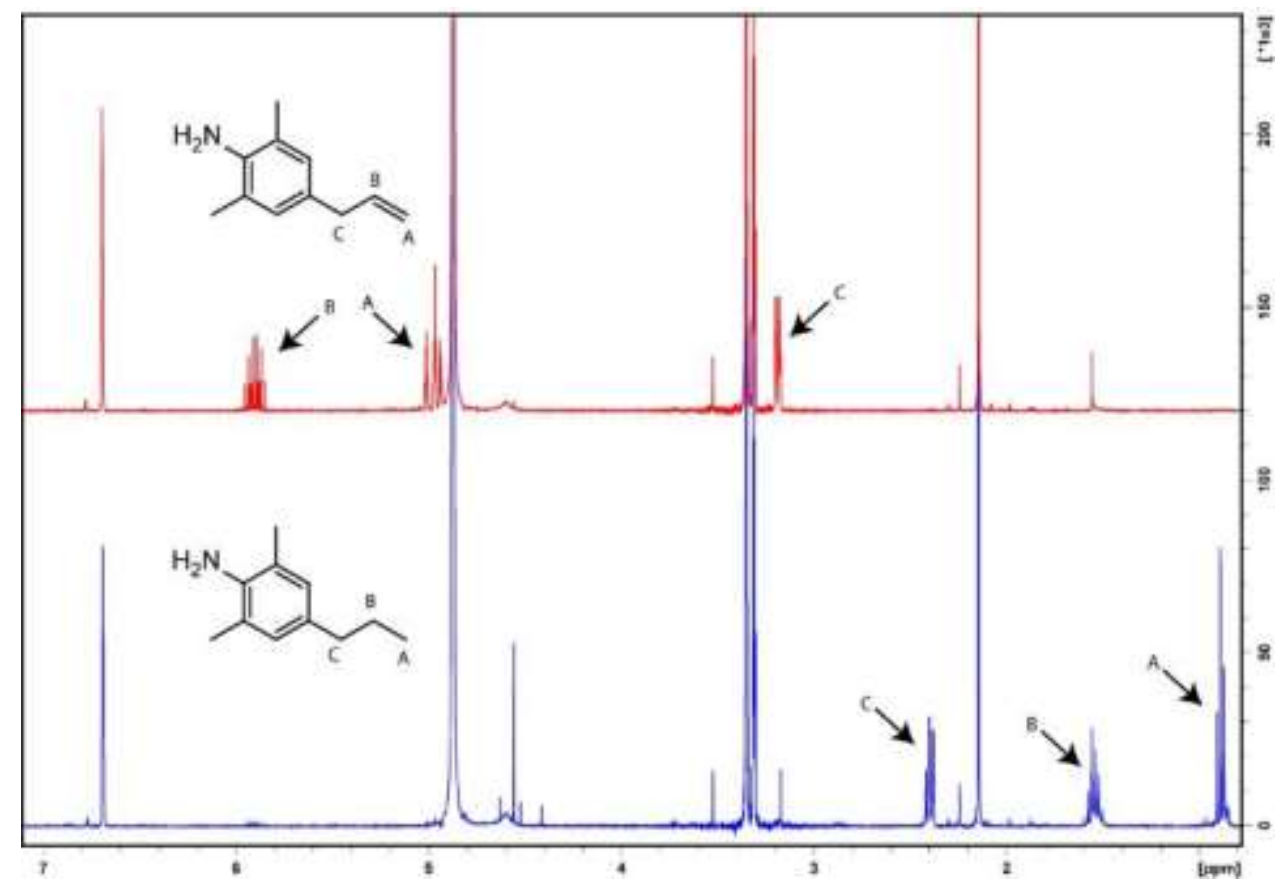

Figure 70: Hydrogenation of the double bond in 4-allyl-2,6-dimethylaniline to a single bond to give 2,6dimethyl-4-propylaniline. 


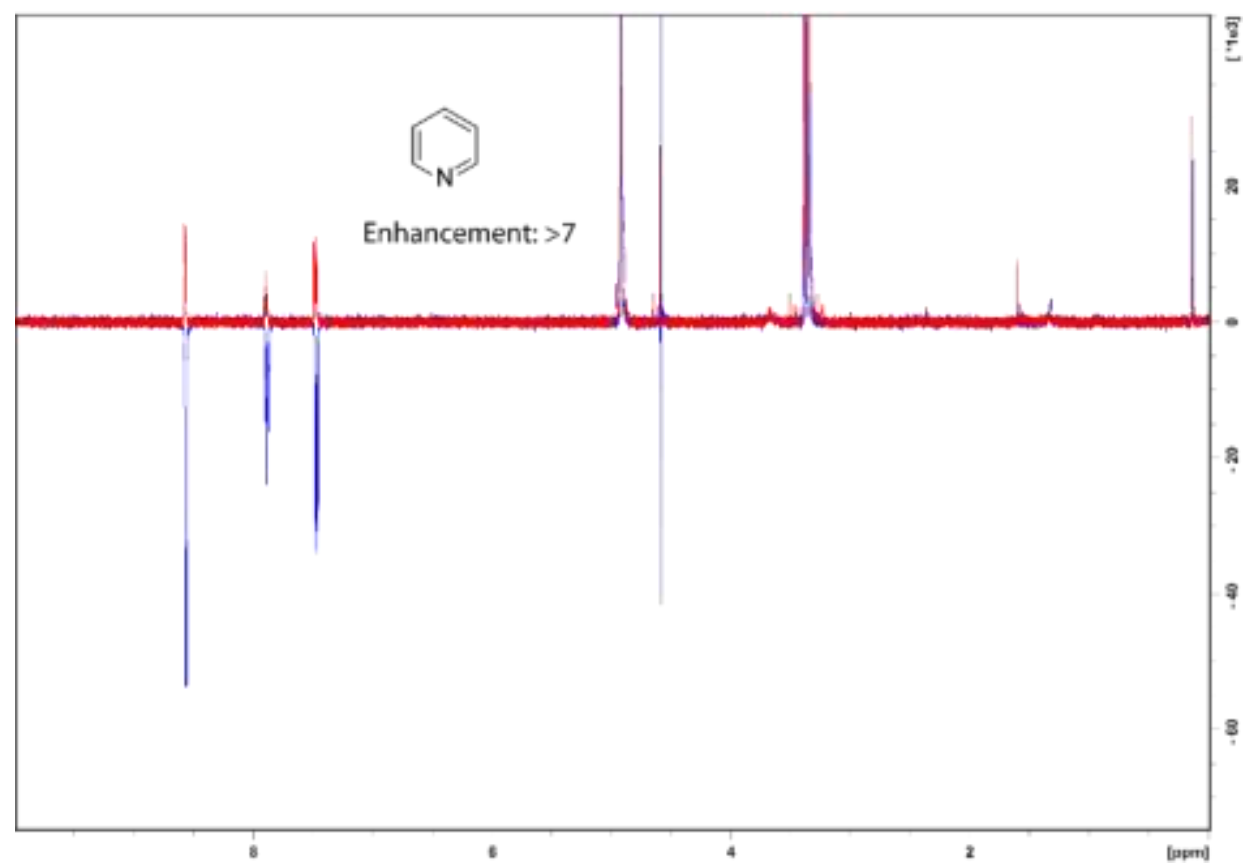

Figure 71: Spectra recorded in SABRE experiments using solid-phase bound catalyst 20 and pyridine as the analyte. The red spectrum displays the thermal equilibrium reference, while the blue spectrum was recorded after exposure of the sample solution and the insoluble catalyst particles to a flow of parahydrogen gas for 30 seconds. 


\section{Materials and Methods}

\subsection{Hyperpolarization Experiments}

\subsubsection{Materials}

All NMR solvents for the hyperpolarization experiments were purchased from Deutero $\mathrm{GmbH}$ with a degree of deuteration of at least $99.8 \%$. The employed precatalysts were synthesized according to literature reported procedures. All examined substrates were purchased from Sigma Aldrich and used without further purification. Pressurized hydrogen and nitrogen gas was purchased from Westfalen $A G$ in 5.0 purity.

\subsubsection{Preparation of Parahydrogen}

The parahydrogen required for conducting the hyperpolarization experiments was prepared using the commercially available Bruker Para Hydrogen Generator BPHG 90. The device is equipped with a cryo-cooler unit that was used to cool high purity $\mathrm{H}_{2}$ gas to temperatures of $36 \mathrm{~K}$. The refrigerated gas was then passed through a conversion chamber filled with a suitable paramagnetic catalyst to facilitate the necessary spin conversion of the $\mathrm{H}_{2}$ molecules from ortho to para. In this way, the hydrogen gas was enriched in $\mathrm{pH}_{2}$ to a level of approximately $92 \%$. The generator requires an $\mathrm{H}_{2} 5.0$ supply of 6 to 10 bar and can be used to fill attached containers with target pressures of up to 6 bar in the "batch delivery more". In "continuous flow mode", the generator can maintain a flow rate of up to $0.2 \mathrm{NL} / \mathrm{min}$ to be fed into an attached consuming device.

\subsubsection{Setup 1: The Shaking Table}

The previously employed setup for conducting the hyperpolarization experiments [35, 122] exposed several severe drawbacks, e.g., polarization field inconsistencies due to the manually executed polarization step and variations in the sample resting time. In order to overcome these, a revised setup was planned and realized in cooperation with the in-house fine mechanics workshop.

\subsubsection{Experimental Setup}

A non-magnetic, tablelike construction was built from aluminum parts in order to support a beam $180 \mathrm{~cm}$ in length running parallel to the ground at a height of approx. $120 \mathrm{~cm}$. In addition, a moveable platform was constructed that could be shifted along the length of the top beam and fixed in position using a positioning screw (Figure 72). The platform was equipped with a mounting for a $5 \mathrm{~mm}$ Wilmad-LabGlass Quick Pressure Valve NMR tube, which in turn was mounted on a metal rail. Thereon, it could be moved from side to side, resulting in a motion perpendicular to the length of the top beam. In order to propel this motion, an electromotor was positioned on the platform and connected to the mounting via a crank. An adjustable power supply was used to power the motor in order to control the speed of the reciprocating motion. 

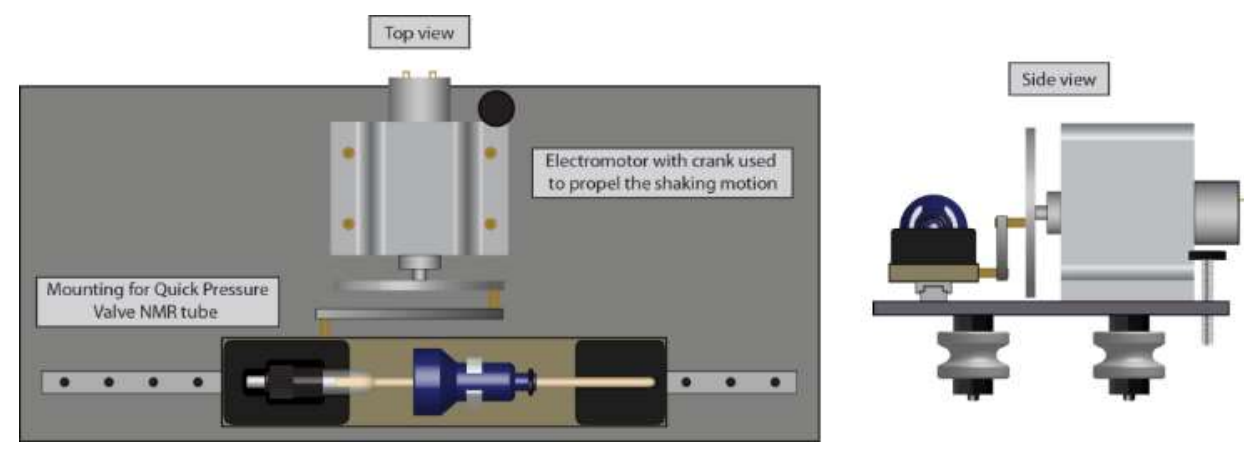

Figure 72: Movable platform holding a sample mounting and the electromotor used to propel the reciprocating motion.

The table construction was placed at a Bruker $600 \mathrm{MHz}$ Avance III spectrometer operating at a ${ }^{1} \mathrm{H}$ base frequency of $600.250 \mathrm{MHz}$ in such a fashion that one end of the top beam was in close contact with the outer shell of the magnet (Figure 73). The length of the beam ran perpendicular to the outer shell, so that the opposing end of the beam was at the furthest possible distance from the center of the magnet. This ensured the accessibility of the highest and lowest possible field strengths in the stray field of the spectrometer.

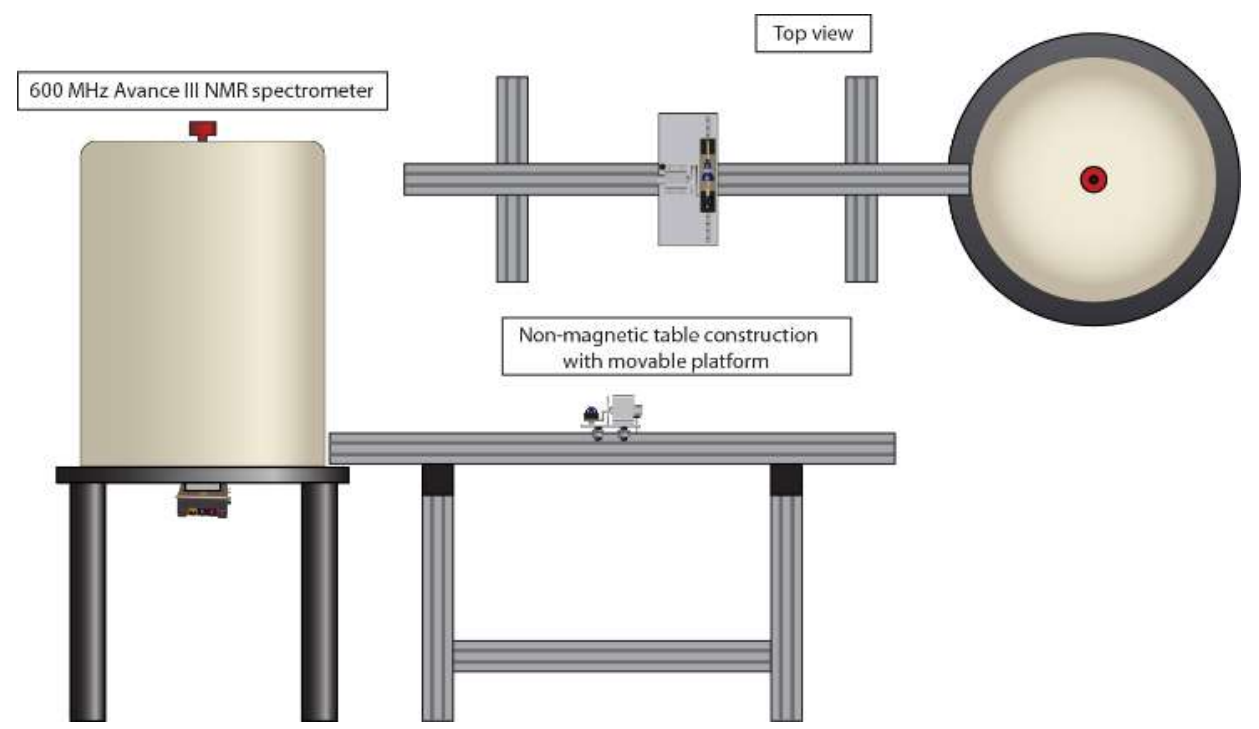

Figure 73: Position of the table construction at the $600 \mathrm{MHz}$ spectrometer. The platform on top of the table was movable along the length of the top beam, allowing for the positioning of the sample at various field strengths within the stray field of the NMR magnet.

A Brockhaus 455 DSP gauss meter was used to determine a series of positions along the length of the top beam, at which the center of the sample tube mounting was located at a certain magnetic field strength (Figure 74). These markings were used to set the magnetic field at which the hyperpolarization experiments were conducted. 


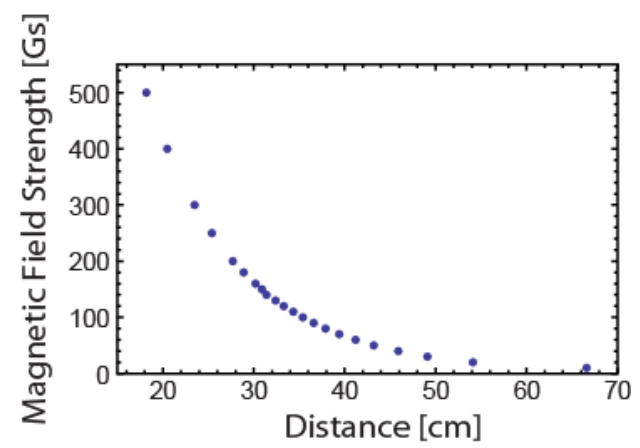

Figure 74: Strength of the magnetic stray field generated by the Bruker Avance III $600 \mathrm{MHz}$ magnet in dependence of the distance from the spectrometer hull.

A non-magnetic step ladder was positioned next to the NMR magnet and the aluminum table to allow for a quick access to the rider holding the sample as well as to the sample inlet at the top of the magnet. A light sensor was attached to the top handle of the ladder and connected to the IPSO board of the NMR console. Covering the sensor resulted in a trigger signal being sent to the spectrometer, aiding in the execution of the pulse sequence. This modification served the purpose of shortening and equalizing the sample resting time inside the spectrometer between its arrival in the magnet and the beginning of the measurement.

\subsubsection{Sample Preparation}

Typical samples consisted of $600 \mu \mathrm{L}$ of methanol- $d_{4}\left(\mathrm{CD}_{3} \mathrm{OD}\right), 1.05 \mathrm{mg}$ of the deuterated precatalyst $\left[\operatorname{Ir}(\mathrm{COD})\left(\mathrm{PC}_{3}-d_{33}\right)\left(\mathrm{py}-d_{5}\right)\right] \mathrm{BF}_{4}(2.23 \mathrm{mM})$ as well as a 9:1 mixture of pyridine- $d_{5}$ and the substrate compound with a total concentration of $22.3 \mathrm{mM}$. This mixture was used as preliminary experiments indicated an increase of observable signal enhancement for the examined substrate when diluted with a secondary, NMR-silent substrate while maintaining the total substrate concentration.

To prepare the sample, the precatalyst was dissolved in $400 \mu \mathrm{L}$ of $\mathrm{CD}_{3} \mathrm{OD}$, followed by addition of $200 \mu \mathrm{L}$ of a $67 \mathrm{mM}$ substrate mixture stock solution in the same solvent via micropipette. The sample solution was transferred into a $5 \mathrm{~mm}$ Wilmad-LabGlass Quick Pressure Valve NMR tube and cooled to $-78^{\circ} \mathrm{C}$ using an acetone / dry ice bath. The tube was then evacuated, pressurized with 4.55 bar of $\mathrm{pH}_{2}$ enriched hydrogen gas, sealed and thawed. Hydrogen gas from the head space over the solution was solvated in the liquid by vigorous shaking of the sample tube, facilitating the reaction between $\mathrm{H}_{2}$ molecules and the iridium precatalyst, resulting in the formation of the active catalyst species $\left[\operatorname{Ir}\left(\mathrm{H}_{2}\right)_{2}\left(\mathrm{PCy}_{3}-\mathrm{d}_{33}\right)(\text { substrate })_{3}\right]\left[\mathrm{BF}_{4}\right]$ under emission of cyclooctane.

\subsubsection{Experimental Procedure}

The sample heater and the related gas flow were turned off during the measurement series in order to avoid the occurrence of a temperature gradient due to a delayed response of the sample heater and the relatively short time between sample insertion and signal acquisition. The NMR experiments were therefore recorded at ambient temperature of approx. $295 \mathrm{~K}$. 
Tuning and matching, automatic adjustment of the NMR shim coils and determination of the $90^{\circ}{ }^{1} \mathrm{H}$ hard pulse was performed before each series of measurements. Typically, three reference spectra were recorded, one each at the beginning, in the course, and at the end of a measurement series.

For each magnetic field strength, the sample was cooled to $-78^{\circ} \mathrm{C}$, evacuated and pressurized with 4.55 bar of $p \mathrm{H}_{2}$ enriched $\mathrm{H}_{2}$ gas. The sample was then thawed, fitted into a plastic spinner and placed in the sample mounting on the rider, which had previously been positioned at the marker for the desired magnetic field and locked in position using the positioning screw.

The zg.ebdu_trigin pulse program was executed before the start of each individual measurement so that the critical components of the spectrometer were given the necessary time to initialize. The program then automatically stopped before execution of the pulse sequence, requiring a trigger input in order to proceed.

Activation of the power supply caused the electromotor to perform the previously described shaking motion, causing a mixing process and a subsequent solvation of the $\mathrm{H}_{2}$ gas in the liquid phase. After deactivation of the power supply, the sample was removed from the clasp and, following a quick adjustment of the spinner position, rapidly inserted into the magnet. When the sample had audibly set inside the magnet, the light sensor was covered so that the pulse sequence was executed. Thereby, the spectrum was immediately recorded using a $90^{\circ}$ proton hard pulse, followed by signal acquisition.

The hyperpolarization experiment was performed three times at each magnetic field strength. After each set of measurements an automatic shimming procedure was executed.

\subsubsection{Evaluation}

Signal intensities were determined by two-point integration of the respective resonances using Bruker TopSpin 3.2. The enhancement factors were determined by

$$
E=\frac{\bar{I}_{p o l}}{\bar{I}_{\text {ref }}}
$$

where $\bar{I}_{r e f}$ and $\bar{I}_{p o l}$ are the dimensionless mean values of the absolute integrals in reference and hyperpolarized spectra, respectively. Experimental errors were determined by calculation of the standard deviation of the enhancement over the number of conducted experiments. 


\subsubsection{Setup 2: The Bruker Para-Hydrogen Polariser}

Due to a number of severe drawbacks of the shaking table setup, e.g., inconsistent transfer times and severe line broadening, this experimental setup was decommissioned and replaced by a more sophisticated one. A prototype of the newly developed Bruker ParaHydrogen Polariser in combination with a flow injection probe was made available for use on this project and implemented as the new hyperpolarization setup.

\subsubsection{Experimental Setup}

The Para-Hydrogen Polariser mainly consists of two parts: the controlling unit and the mixing chamber positioned inside an electromagnetic coil. Both of these parts are attached to a small, non-magnetic table (Figure 75). The controller unit constitutes the main part of the polarizer and houses the valves and gauges necessary to automatically control the various gas flows and pressures during the experiments. It is further equipped with a mechanically operated syringe that is used to load samples from attached sample containers into the mixing chamber and to expel them again. To be operational, the controller must be connected to a nitrogen gas source of between 3 and 6 bar as well as a source of para-enriched hydrogen gas at a pressure between 3 and 6.5 bar. Furthermore, it can be connected to the IPSO board of the NMR console to enable control via the Bruker TopSpin software and therein executed pulse programs.

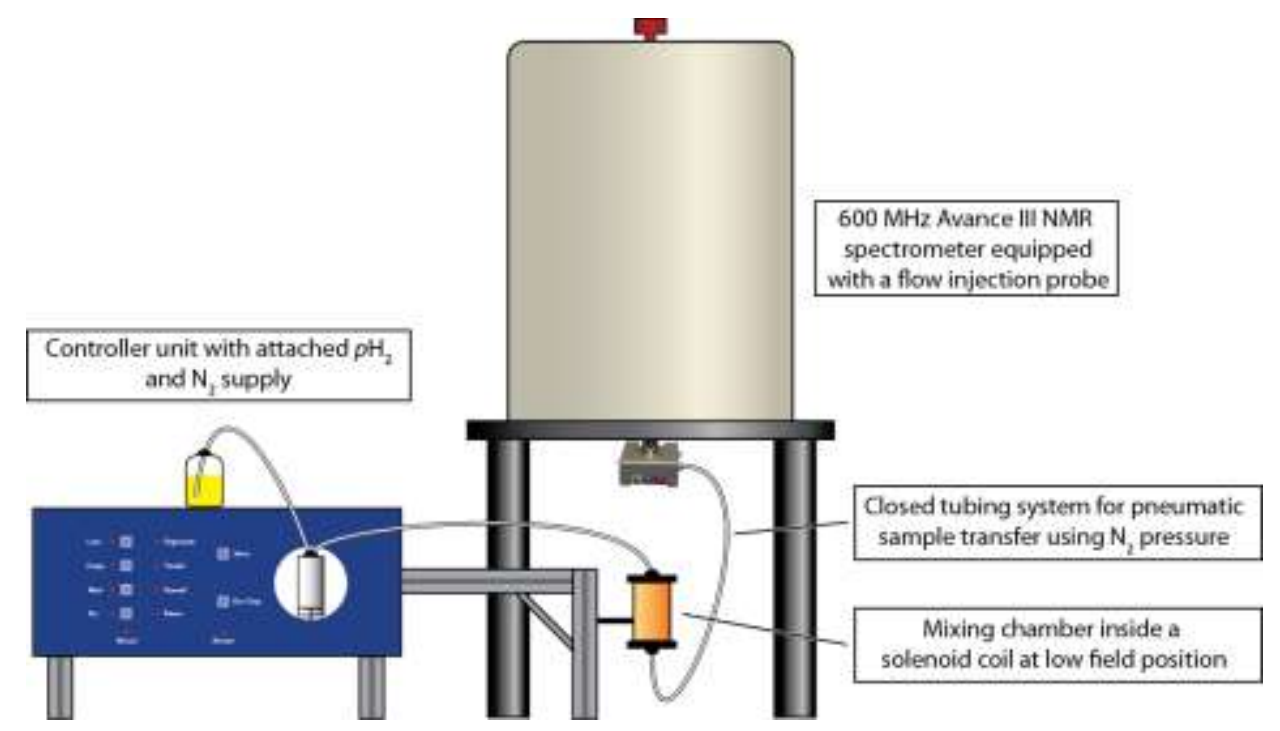

Figure 75: Simplified sketch of experimental setup 2 using the Bruker Para-Hydrogen Polariser and a Bruker Avance III $600 \mathrm{MHz}$ spectrometer.

The mixing chamber constitutes the part of the setup at which the $\mathrm{pH}_{2}$-induced polarization transfer takes place (Figure 76). When loaded with a suitable sample, a flow of para enriched $\mathrm{H}_{2}$ gas can be applied to the chamber through an immersion tube against a lower back pressure above the solution. The pressure difference causes the formation of bubbles from a porous ceramic membrane attached to the lower end of the tube, which rise through the sample solution, solvating $\mathrm{pH}_{2}$ for the polarization transfer. 
The mixing chamber is positioned inside the inner cavity of a solenoid coil, to which adjustable currents can be applied. A magnetic field directly proportional to the current intensity is generated in and around the coil, which either adds to or subtracts from the residual magnetic field present at the location of the coil, depending on the relative alignment of the two magnetic fields. This allows for the polarization step to be performed at varying magnetic fields without the need to physically move the setup to different positions. The position of coil and mixing chamber can, however, be adjusted vertically to allow for the coverage of different ranges of magnetic fields, depending on the prevalent stray field at the chosen height.

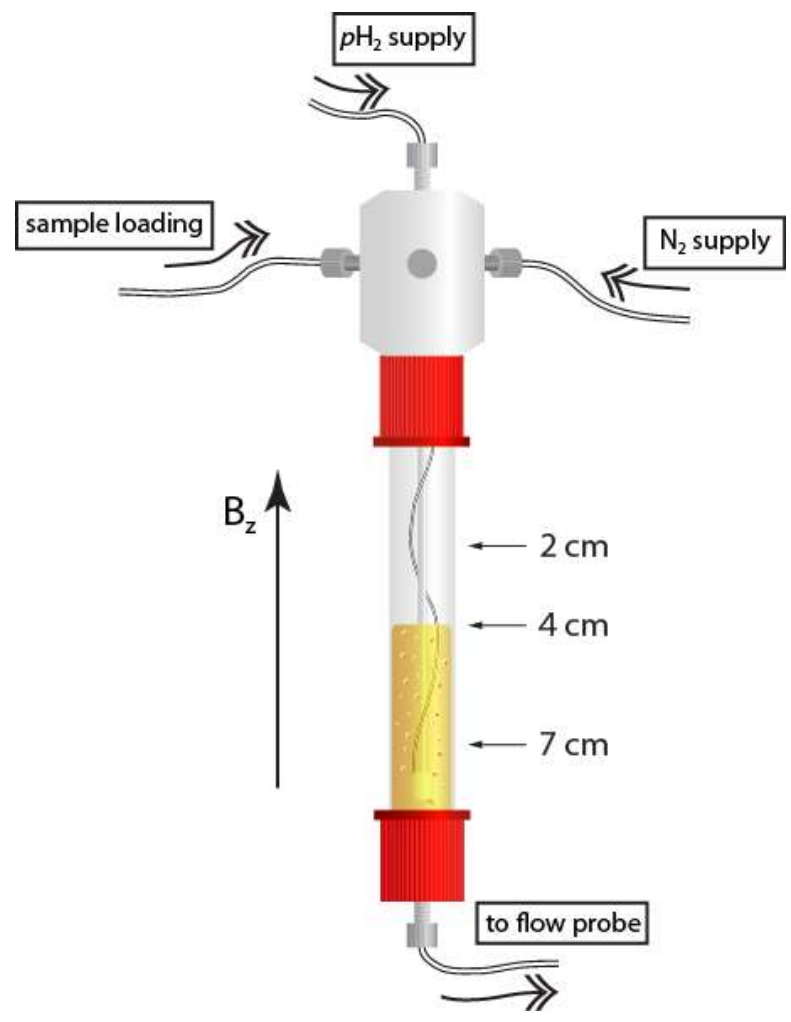

Figure 76: The mixing chamber of the Para-Hydrogen Polariser with all attached tubing connections. The height marks denote the distance from the upper rim auf the electromagnetic coil inside which the mixing chamber is placed. $B_{z}$ denotes the $z$-component of the magnetic field generated by the NMR magnet as well as by the solenoid coil surrounding the mixing chamber.

The individual components that come into contact with the sample solution are connected using capillary tubing with an inner diameter of $0.8 \mathrm{~mm}$. Two different methods for transportation of fluids through the tubing system are used in this setup:

The aforementioned syringe is used for loading and emptying the mixing chamber with either sample solutions or washing solvents at ambient pressure. A selection valve allows for switching between several attached containers. To load a liquid into the syringe, the plunger is retracted using a step motor, creating a suction effect in the applicable vial, transferring the fluid into the syringe. The attached containers are open to the environment to allow for pressure equilibration. To transfer a liquid out of the syringe, the plunger is reinserted into 
the syringe barrel, pushing the fluid through the selection valve and into the desired container, e.g., the mixing chamber.

In order to move a sample from inside the mixing chamber to the flow injection probe, the entire tubing system is pressurized using the connected $N_{2}$ supply. The sample is then transferred by differences between the gas pressures in three successive steps, each of which is defined by a set of meticulously determined transfer parameters, i.e., transfer time, transfer pressure and back pressure. The transfer back into the mixing chamber is achieved in a similar fashion, yet without the critical timing of the transfer parameters.

The Para-Hydrogen Polariser was positioned at a Bruker $600 \mathrm{MHz}$ Avance III spectrometer operating at a ${ }^{1} \mathrm{H}$ base frequency of $600.250 \mathrm{MHz}$ equipped with a $4 \mathrm{~mm}$ FISEI600SB $1 \mathrm{H}-13 \mathrm{C} / \mathrm{D}$ flow probe.

\subsubsection{Determination of Transfer Parameters}

To determine the transfer parameters, the tubing system was connected to a so-called dummy flow cell instead of the flow injection probe (Figure 77). Its specifications, i.e., cell volume and tubing length, were supposedly identical to those of the flow probe, with the distinct advantage of being positioned outside of the NMR magnet, allowing for the direct observation of the achieved filling levels.

As mentioned above, the sample transfer is segmented into three distinct transfer phases. Each of these phases is governed by its transfer parameters, which determine for how long and at which pace the sample solution is propelled through the tubing system. The transfer speed in the individual transfer steps is regulated by the difference between the transfer pressure, which pushes the sample forward, and the back pressure, against which the sample has to be pushed. The transfer time simply denotes for how long the applied pressures are kept up.

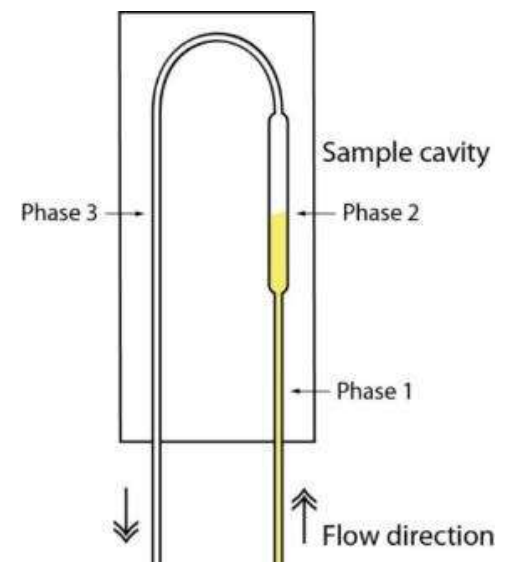

Figure 77: Sketch of the dummy flow cell used to determine the transfer parameters. The arrow markings indicate the optimal liquid level at the end of the respective transfer phase.

In the first phase, the sample is rapidly accelerated towards the flow cell. This phase serves the purpose of covering the relatively long distance between the mixing chamber and the 
sample cavity in the probe. As this path consists solely of the aforementioned $0.8 \mathrm{~mm}$ tubing, the liquid can be pushed with the highest possible pressure difference in order to minimize the transfer time and the loss of hyperpolarization without running the risk of bubble formation in the narrow path. When the liquid level reaches a position few $\mathrm{cm}$ short of the sample cavity (Figure 77), the transfer time for the first phase has been adequately determined.

For the second transfer phase, the sample solution must be slowed down significantly. Upon transition to the flow cavity, the fluid path widens and with too high of a transfer speed, the liquid squirts into the cavity in a narrow jet. This can cause an inclusion of gas bubbles in the lower part of the cavity, leading to an inhomogeneously filled flow cell and ultimately resulting in distorted NMR signals. To avoid this, the pressure difference is reduced by raising the back pressure, causing a deceleration of the liquid and facilitating a slower, but homogenous filling of the cavity from the bottom end. The transfer time is set so that the liquid level at the end of the second phase is at half height of the flow cell (Figure 77).

At this point, the risk of bubble formation is averted, and the sample can be accelerated again to fill the cavity as quickly as possible in order to minimize the loss of hyperpolarization. This is achieved by increasing the transfer pressure while maintaining the previously set back pressure. Lowering the back pressure while maintaining the transfer pressure would cause the same effect on the transfer speed but is avoided in order to prevent effervescence of the solvated $\mathrm{H}_{2}$ gas. The transfer time is adjusted so that the cavity is overfilled, and the final liquid level reaches the opposing side of the flow path (Figure 77) to account for possible fluctuations in the sample transfer.

When the transfer parameters are determined, and the transfers are consistent for the dummy flow cell, the tubing is connected to the flow injection probe. The sample is transferred into the probe and a proton spectrum is recorded using the $z g$ pulse program. The line shape and line width are compared to a previously recorded proton reference spectrum, for which the flow probe had been carefully filled via a manually operated syringe. If the signal quality between the spectra differs wildly, the transfer parameters are adjusted in small increments until the signal quality is satisfactory. Under optimal conditions, the sample can be injected into the flow injection probe in less than $2 \mathrm{~s}$.

\subsubsection{Determination of Polarization Field}

The induced magnetic field inside the cavity of the electromagnetic coil is highly homogenous and runs exclusively along the z-axis of the NMR magnet. Accordingly, only the $z$-component of the residual NMR stray field can be modulated. Therefore, the coil is positioned directly below the center of the NMR magnet where the $x$ - and $y$-components of the stray field are minimal and the strength of the effective magnetic field can be satisfactorily assessed by determination of the z-component alone.

The strength of the electric current fed into the electromagnetic coil is not directly adjustable by selecting the amperage but is instead converted from the approximate 
magnetic field strength to be induced. The selectable values ranged from -145 to $145 \mathrm{Gs}$. As mentioned above, the selected field value did not correspond to the effective magnetic field inside the mixing chamber, but to the magnetic field strength that was added to or subtracted from the prevalent field at the position of the chamber. Previously published results [35, 122] determined the field strengths of interest for this hyperpolarization technique to span from 0 to $250 \mathrm{Gs}$, indicating an optimal residual field at the coil position of between 105 and $145 \mathrm{Gs}$ to ensure coverage of this field range.

The height of the coil was set to the lowest possible position on the vertical beam owing to the relatively strong stray field emitted by the $141 \mathrm{kGs}$ NMR magnet. With this setting, a field strength of $113 \mathrm{Gs}$ was present at half height of the coil.

The effective field under application of various currents to the electromagnetic coil was determined at three distinct depths inside of the coil cavity using a Brockhaus 455 DSP gauss meter with an axial probe. These depths were chosen based on occurring liquid levels during a series of experiments: When a typical sample volume is loaded into the mixing chamber, the fill level is at a depth of $4 \mathrm{~cm}$ from the upper rim of the coil, with the center of the sample volume being at a depth of around $7 \mathrm{~cm}$ (Figure 77). During the polarization step, when the sample is interspersed with gas bubbles, the effective sample volume increases significantly to a fill level of only $2 \mathrm{~cm}$ into the cavity from the top end. Due to the steep stray field gradient, the effective fields at these three positions diverge significantly (Figure 78). Additionally, the applied field at a depth of $2 \mathrm{~cm}$ from the upper rim of the coil appears to not run homogenously along the $z$-axis anymore, but to exhibit also strong $x$ - and $y$ components, thereby limiting the effectiveness of the field modulation. This is indicated by the divergent slope of the line through the data points at this depth compared to positions deeper into the cavity.

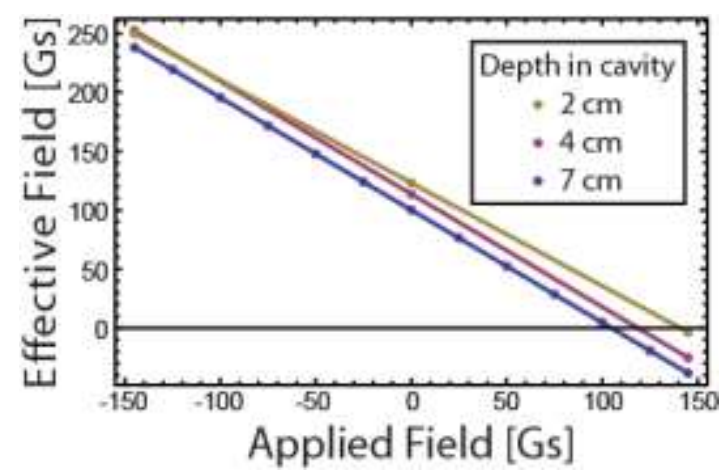

Figure 78: Effective polarization field at different positions inside the mixing chamber in dependence of the magnetic field induced by the electromagnetic coil.

Over the course of an experiment series, the sample volume decreased, and the liquid level no longer reached the $4 \mathrm{~cm}$ marking. As the sample did, however, constantly cover the marking at a depth of $7 \mathrm{~cm}$, the field strengths determined at this position were selected as the reference points. The individual effective field strengths were calculated using the line equation obtained from a linear regression 


$$
B_{\text {eff }}=-0.9531 \times B_{\text {mod }}+100.1
$$

with $B_{\text {eff }}$ being the effective field strength and $B_{\text {mod }}$ being the modulation field generated by the electromagnetic coil.

\subsubsection{Sample Preparation}

Typical samples consisted of $3.6 \mathrm{~mL}$ of methanol- $d_{4}\left(\mathrm{CD}_{3} \mathrm{OD}\right), 4.04 \mu \mathrm{mol}$ of a suitable precatalyst $(1.12 \mathrm{mM})$ and $40.4 \mu \mathrm{mol}$ of the substrate compound $(11.2 \mathrm{mM})$. The precatalyst was weighed into a $4 \mathrm{~mL}$ glass vial and solvated in $2 \mathrm{~mL}$ of $\mathrm{CD}_{3} \mathrm{OD}$. The substrate was weighed into an Eppendorf cup and dissolved in $800 \mu \mathrm{L}$ of the solvent. The substrate solution was then added into the sample vial and the Eppendorf cup was rinsed with additional $800 \mu \mathrm{L}$ of solvent, which were also transferred into the sample container. The vial was sealed with a screw-on septum cap and vortexed until the metal complex was dissolved.

The sample vial was attached to the Bruker Para-Hydrogen Polariser via the sample loading capillary and $3.1 \mathrm{~mL}$ of the sample solution were transferred into the mixing chamber via the implemented syringe. Parahydrogen was applied to the mixing chamber for several seconds to facilitate the reaction between $\mathrm{H}_{2}$ molecules and the iridium precatalyst, resulting in the formation of the active catalyst under emission of cyclooctane.

\subsubsection{Experimental Procedure}

After preparation of the active catalyst species, the sample solution was shuttled several times between the flow probe and the mixing chamber. This was done to ensured consistent transfers in the forthcoming experiments, as, after the return transfer, some droplets of the sample solution are not cleared from the tubing system, slightly changing the transfer characteristics. After several transfers, this effect equilibrated.

The sample was then transferred into the flow injection probe to perform the tuning and matching procedure as well as the adjustment of the shim coils. The length of the $90^{\circ}$ proton hard pulse was determined before each set of measurements. The temperature unit was set to the approximate ambient temperature of $295 \mathrm{~K}$.

Hyperpolarization experiments were executed using the ph_zg_noload_noempty pulse program. Using the connection of the Para-Hydrogen Polariser to the IPSO board, this pulse program is able to control the entire experiment including the polarization step, sample transfer, acquisition and return transfer using various user defined delays and parameters.

Before the start of the field dependence measurements, a set of bubbling time experiments was performed. Therein, the application time of the para enriched $\mathrm{H}_{2}$ gas was varied from 2 to $24 \mathrm{~s}$ in $2 \mathrm{~s}$ increments to assess an optimal exposure time. This also served the purpose of validating transfer consistency.

Typically, three reference spectra were recorded, one each before the bubble time experiments, before the field dependence experiments and after the measurement series had ended. To do so, the sample was transferred into the probe and allowed to rest for at 
least $60 \mathrm{~s}$ to ensure the decay of any possible hyperpolarization. The reference spectra were recorded using the $z g$ pulse program.

The measurement series was started using the multizg_ph_bo_new macro. When executed, the program prompts for input of the initial applied field strength, the field increment and the number of experiments in order to cover the desired field range in the experimental series. Typical values resulted in coverage of the modulation field range from -145 to $145 \mathrm{Gs}$ in $5 \mathrm{Gs}$ increments, equivalent to effective fields ranging from -38.2 to $138 \mathrm{Gs}$.

The experimental procedure for an individual experiment went as follows: Parahydrogen gas was applied to the immersion tube at a pressure of 3.0 bar against a back pressure of 2.4 bar for the previously determined exposure time. After a delay to allow for the gas bubbles to clear the solution, the sample was transferred into the flow injection probe in the described pneumatic three-step transfer. After a further short delay to reduce sample perturbation, the proton spectrum was recorded using a $90^{\circ}{ }^{1} \mathrm{H}$ hard pulse, followed by acquisition of the FID. The sample was then transferred back into the mixing chamber for execution of the next hyperpolarization experiment.

Following a series of experiment, the sample was removed from the mixing chamber and transferred into a waste container. Subsequently, a repetitive wash operation was executed to flush the tubing system including mixing chamber and flow probe for 10 min using pure methanol. Afterwards, a drying program was run for $30 \mathrm{~min}$, expelling and evaporating all residual liquid inside the tubing system using a flow of $\mathrm{N}_{2}$.

\subsubsection{Evaluation}

Signal intensities were determined by two-point integration of the respective resonances using Bruker TopSpin 3.2. The enhancement factors were determined by

$$
E=\frac{I_{p o l}}{\bar{I}_{r e f}}
$$

where $I_{p o l}$ is the dimensionless absolute integral of the polarized resonance and $\bar{I}_{r e f}$ is the dimensionless mean value of the absolute integrals in the reference spectra.

\subsubsection{NMR Data of Examined Substrates}

\subsubsection{Pyrazole and its related compounds}

pyrazole

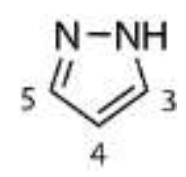

Figure 79: Molecular structure and numbering of the substrate molecule pyrazole.

${ }^{1} \mathrm{H}$ NMR: $\left(600 \mathrm{MHz}, \mathrm{CD}_{3} \mathrm{OD}\right): \delta=7.63 \mathrm{ppm}(\mathrm{sbr}, 2 \mathrm{H}, \mathrm{H} 3 / \mathrm{H} 5), 6.35$ (t, $\left.1 \mathrm{H}, \mathrm{H} 4\right) \mathrm{ppm}$. 
3-methylpyrazole

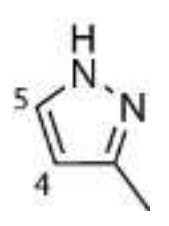

Figure 80: Molecular structure and numbering of the substrate molecule 3-methylpyrazole.

${ }^{1} \mathrm{H}$ NMR: $\left(600 \mathrm{MHz}, \mathrm{CD}_{3} \mathrm{OD}\right): \delta=7.47 \mathrm{ppm}(\mathrm{sbr}, 1 \mathrm{H}, \mathrm{H} 5), 6.08(\mathrm{sbr}, 1 \mathrm{H}, \mathrm{H} 4), 2.30\left(\mathrm{~s}, 3 \mathrm{H}, \mathrm{CH}_{3}\right)$ ppm.

4-methylpyrazole

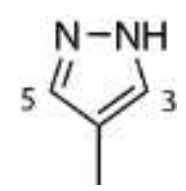

Figure 81: Molecular structure and numbering of the substrate molecule 4-methylpyrazole.

${ }^{1} \mathrm{H}$ NMR: $\left(600 \mathrm{MHz}, \mathrm{CD}_{3} \mathrm{OD}\right): \delta=7.37 \mathrm{ppm}\left(\mathrm{s}_{\mathrm{br}}, 2 \mathrm{H}, \mathrm{H} 3 / \mathrm{H} 5\right), 2.09\left(\mathrm{~s}, 3 \mathrm{H}, \mathrm{CH}_{3}\right) \mathrm{ppm}$.

3,4-dimethylpyrazole

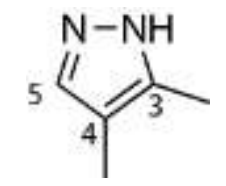

Figure 82: Molecular structure and numbering of the substrate molecule 3,4-dimethylpyrazole.

${ }^{1} \mathrm{H}$ NMR: (600 MHz, CD $\left.{ }_{3} \mathrm{OD}\right): \delta=7.28 \mathrm{ppm}(\mathrm{s}, 1 \mathrm{H}, \mathrm{H} 5), 2.20$ (s, 3H, 3-Me), 2.01 (s, 3H, 4-Me) ppm.

\section{3,5-dimethylpyrazole}

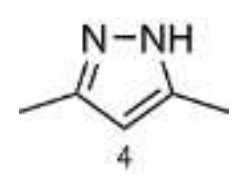

Figure 83: Molecular structure and numbering of the substrate molecule 3,5-dimethylpyrazole.

${ }^{1} \mathrm{H}$ NMR: $\left(600 \mathrm{MHz}, \mathrm{CD}_{3} \mathrm{OD}\right): \delta=5.83 \mathrm{ppm}(\mathrm{s}, 1 \mathrm{H}, \mathrm{H} 4), 2.22\left(\mathrm{~s}, 6 \mathrm{H}, \mathrm{CH}_{3}\right) \mathrm{ppm}$.

3-phenylpyrazole

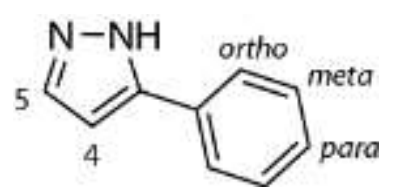

Figure 84: Molecular structure and numbering of the substrate molecule 3-phenylpyrazole.

${ }^{1} \mathrm{H}$ NMR: $\left(600 \mathrm{MHz}, \mathrm{CD}_{3} \mathrm{OD}\right): \delta=7.78 \mathrm{ppm}(\mathrm{sbr}, 2 \mathrm{H}, \mathrm{o}-\mathrm{Ph}), 7.68(\mathrm{sbr}, 1 \mathrm{H}, \mathrm{H} 5), 7.43(\mathrm{t}, 2 \mathrm{H}$, $m-\mathrm{Ph}), 7.34(\mathrm{t}, 1 \mathrm{H}, p-\mathrm{Ph}), 6.68\left(\mathrm{~d}_{\mathrm{br}}, 1 \mathrm{H}, \mathrm{H} 4\right) \mathrm{ppm}$. 
3-(4-aminophenyl)pyrazole

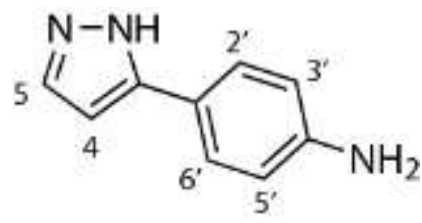

Figure 85: Molecular structure and numbering of the substrate molecule 3-(4-aminophenyl)pyrazole.

${ }^{1}{ }_{\mathrm{H}}$ NMR: $\left(600 \mathrm{MHz}, \mathrm{CD}_{3} \mathrm{OD}\right): \delta=7.57 \mathrm{ppm}\left(\mathrm{s}_{\mathrm{br}}, 1 \mathrm{H}, \mathrm{H} 5\right), 7.49\left(\mathrm{~d}_{\mathrm{br}}, 2 \mathrm{H}, 2^{\prime} / 6^{\prime}\right), 6.77(\mathrm{~d}, 2 \mathrm{H}$, $\left.3^{\prime} / 5^{\prime}\right), 6.49(\mathrm{sbr}, 1 \mathrm{H}, \mathrm{H} 4) \mathrm{ppm}$.

\section{3,5-diphenylpyrazole}

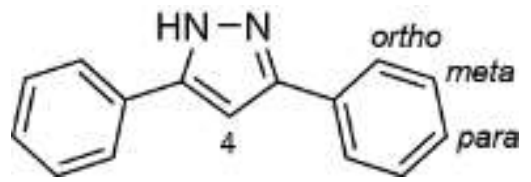

Figure 86: Molecular structure and numbering of the substrate molecule 3,5-diphenylpyrazole.

${ }^{1} \mathrm{H}$ NMR: $\left(600 \mathrm{MHz}, \mathrm{CD}_{3} \mathrm{OD}\right): \delta=7.81 \mathrm{ppm}\left(\mathrm{s}_{\mathrm{br}}, 4 \mathrm{H}, \mathrm{o}-\mathrm{H}\right), 7.46\left(\mathrm{~s}_{\mathrm{br}}, 4 \mathrm{H}, \mathrm{m}-\mathrm{H}\right), 7.37(\mathrm{sbr}, 2 \mathrm{H}$, $p-\mathrm{H}), 7.01(\mathrm{~s}, 1 \mathrm{H}, \mathrm{H} 4) \mathrm{ppm}$.

\section{3,5-ditertbutylpyrazole}

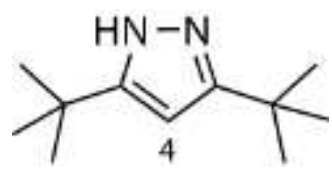

Figure 87: Molecular structure and numbering of the substrate molecule 3,5-ditertbutylpyrazole.

${ }^{1} \mathrm{H}$ NMR: $\left(600 \mathrm{MHz}, \mathrm{CD}_{3} \mathrm{OD}\right): \delta=5.94(\mathrm{~s}, 1 \mathrm{H}, \mathrm{H} 4), 1.31\left(\mathrm{~s}, 18 \mathrm{H}, \mathrm{CH}_{3}\right) \mathrm{ppm}$.

3-aminopyrazole

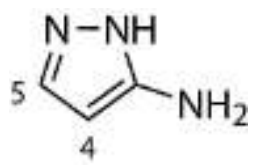

Figure 88: Molecular structure and numbering of the substrate molecule 3-aminopyrazole.

${ }^{1} \mathrm{H}$ NMR: $\left(600 \mathrm{MHz}, \mathrm{CD}_{3} \mathrm{OD}\right): \delta=7.34 \mathrm{ppm}(\mathrm{d}, 1 \mathrm{H}, \mathrm{H} 5), 5.64(\mathrm{~d}, 1 \mathrm{H}, \mathrm{H} 4) \mathrm{ppm}$.

4-ethoxy-3-methylpyrazole

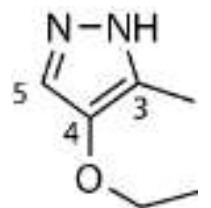

Figure 89: Molecular structure and numbering of the substrate molecule 4-ethoxy-3-methylpyrazole.

${ }^{1} \mathrm{H}$ NMR: $\left(600 \mathrm{MHz}, \mathrm{CD}_{3} \mathrm{OD}\right): \delta=5.49 \mathrm{ppm}(\mathrm{s}, 1 \mathrm{H}, \mathrm{H} 5), 4.09$ (q, $\left.2 \mathrm{H}, \mathrm{OCH}_{2} \mathrm{CH}_{3}\right), 2.22(\mathrm{~s}, 3 \mathrm{H}$, 3-Me), 1.35 (t, $3 \mathrm{H}, \mathrm{OCH}_{2} \underline{\mathrm{CH}_{3}}$ ) ppm. 
indazole

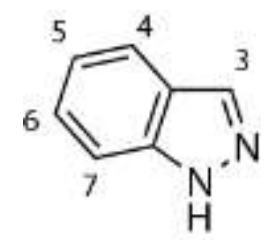

Figure 90: Molecular structure and numbering of the substrate molecule indazole.

${ }^{1} \mathrm{H}$ NMR: $\left(600 \mathrm{MHz}, \mathrm{CD}_{3} \mathrm{OD}\right): \delta=8.05 \mathrm{ppm}(\mathrm{s}, 1 \mathrm{H}, \mathrm{H} 3), 7.79(\mathrm{dt}, 1 \mathrm{H}, \mathrm{H} 4), 7.55$ (dd, $\left.1 \mathrm{H}, \mathrm{H} 7\right)$, 7.40 (ddd, 1H, H6), 7.16 (ddd, 1H, H5) ppm.

1,2,3-triazole<smiles>Cc1csnn1</smiles>

Figure 91: Molecular structure and numbering of the substrate molecule 1,2,3-triazole.

${ }^{1} \mathrm{H}$ NMR: (600 MHz, $\left.\mathrm{CD}_{3} \mathrm{OD}\right): \delta=7.85 \mathrm{ppm}(\mathrm{sbr}, 2 \mathrm{H}, \mathrm{H} 4 / \mathrm{H} 5) \mathrm{ppm}$.

\section{1,2,4-triazole}<smiles>c1nc[nH]n1</smiles>

Figure 92: Molecular structure and numbering of the substrate molecule 1,2,4-triazole.

${ }^{1} \mathrm{H}$ NMR: (600 MHz, $\left.\mathrm{CD}_{3} \mathrm{OD}\right): \delta=8.35 \mathrm{ppm}(\mathrm{sbr}, 2 \mathrm{H}, \mathrm{H} 3 / \mathrm{H} 5) \mathrm{ppm}$.

\subsubsection{Pyridine and its related compounds}

pyridine<smiles>O=Cc1cccnc1O</smiles>

Figure 93: Molecular structure and numbering of the substrate molecule pyridine.

${ }^{1} \mathrm{H}$ NMR: $\left(600 \mathrm{MHz}, \mathrm{CD}_{3} \mathrm{OD}\right): \delta=8.55(\mathrm{~m}, 2 \mathrm{H}, \mathrm{o}-\mathrm{H}), 7.88(\mathrm{tt}, 1 \mathrm{H}, p-\mathrm{H}), 7.46(\mathrm{~m}, 2 \mathrm{H}, m-\mathrm{H}) \mathrm{ppm}$. 2,6-dideuteropyridine<smiles>[2H]c1cccc([2H])n1</smiles>

Figure 94: Molecular structure and numbering of the substrate molecule 2,6-dideuteropyridine.

${ }^{1} \mathrm{H}$ NMR: (600 MHz, CD $\left.30 \mathrm{OD}\right): \delta=7.88(\mathrm{t}, 1 \mathrm{H}, \mathrm{H} 4), 7.46(\mathrm{~d}, 2 \mathrm{H}, \mathrm{H} 3 / \mathrm{H} 5) \mathrm{ppm}$. 


\section{3,4,5-trideuteropyridine}<smiles>[2H]c1cncc([2H])c1[2H]</smiles>

Figure 95: Molecular structure and numbering of the substrate molecule 3,4,5-trideuteropyridine.

${ }^{1} \mathrm{H}$ NMR: $\left(600 \mathrm{MHz}, \mathrm{CD}_{3} \mathrm{OD}\right): \delta=8.53(\mathrm{~s}, 2 \mathrm{H}, \mathrm{H} 2 / \mathrm{H} 6) \mathrm{ppm}$.

nicotinamide<smiles>NC(=O)c1cccnc1</smiles>

Figure 96: Molecular structure and numbering of the substrate molecule nicotinamide.

${ }^{1} \mathrm{H}$ NMR: (600 MHz, $\mathrm{CD}_{3} \mathrm{OD}$ ): $\delta=9.04$ (dd, $1 \mathrm{H}, \mathrm{H} 2$ ), 8.71 (dd, $\left.1 \mathrm{H}, \mathrm{H} 6\right), 8.31$ (ddd, $\left.1 \mathrm{H}, \mathrm{H} 4\right), 7.57$ (ddd, $1 \mathrm{H}, \mathrm{H} 5)$ ppm.

3-fluoropyridine

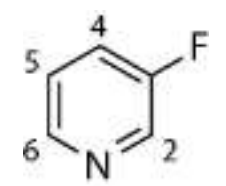

Figure 97: Molecular structure and numbering of the substrate molecule 3-fluoropyridine.

${ }^{1} \mathrm{H}$ NMR: $\left(600 \mathrm{MHz}, \mathrm{CD}_{3} \mathrm{OD}\right): \delta=8.50(\mathrm{~d}, 1 \mathrm{H}, \mathrm{H} 2), 8.43(\mathrm{~m}, 1 \mathrm{H}, \mathrm{H} 6), 7.68$ (ddd, $\left.1 \mathrm{H}, \mathrm{H} 4\right), 7.52$ (ddd, $1 \mathrm{H}, \mathrm{H} 5)$ ppm.

3-chloropyridine<smiles>Clc1ccncc1</smiles>

Figure 98: Molecular structure and numbering of the substrate molecule 3-chloropyridine.

${ }^{1} \mathrm{H}$ NMR: $\left(600 \mathrm{MHz}, \mathrm{CD}_{3} \mathrm{OD}\right): \delta=8.59$ (d, $\left.1 \mathrm{H}, \mathrm{H} 2\right), 8.50$ (dd, 1H, H6), 7.92 (ddd, 1H, H4), 7.46 (ddd, $1 \mathrm{H}, \mathrm{H} 5)$ ppm. 
3-bromopyridine<smiles>Brc1ccncc1</smiles>

Figure 99: Molecular structure and numbering of the substrate molecule 3-bromopyridine.

${ }^{1} \mathrm{H}$ NMR: $\left(600 \mathrm{MHz}, \mathrm{CD}_{3} \mathrm{OD}\right): \delta=8.69$ (d, $1 \mathrm{H}, \mathrm{H} 2$ ), 8.54 (ddd, $\left.1 \mathrm{H}, \mathrm{H} 6\right), 8.06$ (ddd, $\left.1 \mathrm{H}, \mathrm{H} 4\right), 7.41$ (ddd, $1 \mathrm{H}, \mathrm{H} 5)$ ppm.

3-iodopyridine

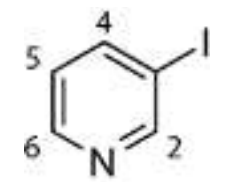

Figure 100: Molecular structure and numbering of the substrate molecule 3-iodopyridine.

${ }^{1} \mathrm{H}$ NMR: $\left(600 \mathrm{MHz}, \mathrm{CD}_{3} \mathrm{OD}\right): \delta=8.82$ (dd, $1 \mathrm{H}, \mathrm{H} 2$ ), 8.55 (dd, $\left.1 \mathrm{H}, \mathrm{H} 6\right), 8.23$ (ddd, $\left.1 \mathrm{H}, \mathrm{H} 4\right), 7.28$ (ddd, $1 \mathrm{H}, \mathrm{H} 5$ ) ppm.

3-methylpyridine

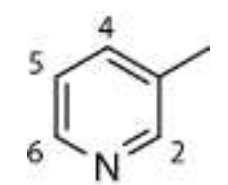

Figure 101: Molecular structure and numbering of the substrate molecule 3-methylpyridine.

${ }^{1} \mathrm{H}$ NMR: $\left(600 \mathrm{MHz}, \mathrm{CD}_{3} \mathrm{OD}\right): \delta=8.40(\mathrm{~m}, 1 \mathrm{H}, \mathrm{H} 2), 8.35(\mathrm{~m}, 1 \mathrm{H}, \mathrm{H} 6), 7.71(\mathrm{~m}, 1 \mathrm{H}, \mathrm{H} 4), 7.35$ (m, 1H, H5), $2.39\left(\mathrm{~s}, 3 \mathrm{H}, \mathrm{CH}_{3}\right) \mathrm{ppm}$.

3-methoxypyridine<smiles>COc1cccnc1</smiles>

Figure 102: Molecular structure and numbering of the substrate molecule 3-methoxypyridine.

${ }^{1} \mathrm{H}$ NMR: $\left(600 \mathrm{MHz}, \mathrm{CD}_{3} \mathrm{OD}\right.$ ): $\delta=8.25$ (d, $1 \mathrm{H}, \mathrm{H} 2$ ), 8.14 (dd, $\left.1 \mathrm{H}, \mathrm{H} 6\right), 7.46$ (ddd, $\left.1 \mathrm{H}, \mathrm{H} 4\right), 7.40$ (ddd, $1 \mathrm{H}, \mathrm{H} 5), 3.90\left(\mathrm{~s}, 3 \mathrm{H}, \mathrm{CH}_{3}\right) \mathrm{ppm}$.

3-hydroxypyridine<smiles>Oc1ccncc1</smiles>

Figure 103: Molecular structure and numbering of the substrate molecule 3-hydroxypyridine. 
${ }^{1} \mathrm{H}$ NMR: $\left(600 \mathrm{MHz}, \mathrm{CD}_{3} \mathrm{OD}\right): \delta=8.10$ (dd, $1 \mathrm{H}, \mathrm{H} 2$ ), 8.02 (dd, $\left.1 \mathrm{H}, \mathrm{H} 6\right), 7.29$ (ddd, $\left.1 \mathrm{H}, \mathrm{H} 4\right), 7.26$ (ddd, $1 \mathrm{H}, \mathrm{H} 5)$ ppm.

\section{3,5-dichloropyridine}<smiles>Clc1cncc(Cl)c1</smiles>

Figure 104: Molecular structure and numbering of the substrate molecule 3,5-dichloropyridine.

${ }^{1} \mathrm{H}$ NMR: $\left(600 \mathrm{MHz}, \mathrm{CD}_{3} \mathrm{OD}\right): \delta=8.54(\mathrm{~s}, 2 \mathrm{H}, \mathrm{H} 2 / \mathrm{H} 6), 8.05(\mathrm{~s}, 1 \mathrm{H}, \mathrm{H} 4) \mathrm{ppm}$.

\section{3,5-dibromopyridine}<smiles>Brc1cncc(Br)c1</smiles>

Figure 105: Molecular structure and numbering of the substrate molecule 3,5-dibromopyridine.

${ }^{1} \mathrm{H}$ NMR: $\left(600 \mathrm{MHz}, \mathrm{CD}_{3} \mathrm{OD}\right): \delta=8.66(\mathrm{~d}, 2 \mathrm{H}, \mathrm{H} 2 / \mathrm{H} 6), 8.32(\mathrm{t}, 1 \mathrm{H}, \mathrm{H} 4) \mathrm{ppm}$.

\section{3,5-dimethylpyridine}

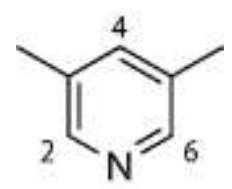

Figure 106: Molecular structure and numbering of the substrate molecule 3,5-dimethylpyridine.

${ }^{1} \mathrm{H}$ NMR: $\left(600 \mathrm{MHz}, \mathrm{CD}_{3} \mathrm{OD}\right): \delta=8.19(\mathrm{~m}, 2 \mathrm{H}, \mathrm{H} 2 / \mathrm{H} 6), 7.54(\mathrm{~m}, 1 \mathrm{H}, \mathrm{H} 4), 2.34\left(\mathrm{~d}, 6 \mathrm{H}, \mathrm{CH}_{3}\right)$ ppm.

\section{3,4-dimethylpyridine}

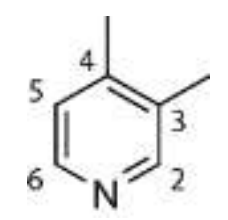

Figure 107: Molecular structure and numbering of the substrate molecule 3,4-dimethylpyridine.

${ }^{1} \mathrm{H}$ NMR: $\left(600 \mathrm{MHz}, \mathrm{CD}_{3} \mathrm{OD}\right): \delta=8.26(\mathrm{~s}, 1 \mathrm{H}, \mathrm{H} 2), 8.22(\mathrm{~d}, 1 \mathrm{H}, \mathrm{H} 6), 2.35$ (d, 3H, 3-CH 3 ), 2.31 (s, $\left.3 \mathrm{H}, 4-\mathrm{CH}_{3}\right) \mathrm{ppm}$. 
pyridazine<smiles>c1ccncc1</smiles>

Figure 108: Molecular structure and numbering of the substrate molecule pyridazine.

${ }^{1} \mathrm{H}$ NMR: $\left(600 \mathrm{MHz}, \mathrm{CD}_{3} \mathrm{OD}\right): \delta=9.22(\mathrm{t}, 2 \mathrm{H}, \mathrm{H} 3 / \mathrm{H} 6), 7.77(\mathrm{t}, 2 \mathrm{H}, \mathrm{H} 4 / \mathrm{H} 5) \mathrm{ppm}$.

quinoline<smiles>c1ccc2ncccc2c1</smiles>

Figure 109: Molecular structure and numbering of the substrate molecule quinoline.

${ }^{1} \mathrm{H}$ NMR: $\left(600 \mathrm{MHz}, \mathrm{CD}_{3} \mathrm{OD}\right): \delta=8.88$ (dd, $1 \mathrm{H}, \mathrm{H} 2$ ), 8.41 (d, $\left.1 \mathrm{H}, \mathrm{H} 8\right), 8.06$ (d, 1H, H4), 7.99 (d, $1 \mathrm{H}, \mathrm{H5}$ ), 7.82 (ddd, $1 \mathrm{H}, \mathrm{H7}$ ), 7.66 (ddd, $1 \mathrm{H}, \mathrm{H6}$ ), 7.58 (dd, 1H, H3) ppm.

4-bromo-2,8-di(trifluoromethyl)quinoline<smiles>FC(F)(F)c1cc(Br)c2cccc(C(F)(F)F)c2n1</smiles>

Figure 110: Molecular structure and numbering of the substrate molecule 4-bromo-2,8-di(trifluoromethyl)quinoline.

${ }^{1} \mathrm{H}$ NMR: $\left(600 \mathrm{MHz}, \mathrm{CD}_{3} \mathrm{OD}\right): \delta=8.64(\mathrm{~d}, 1 \mathrm{H}, \mathrm{H} 5), 8.40(\mathrm{~s}, 1 \mathrm{H}, \mathrm{H} 3), 8.38(\mathrm{~d}, 1 \mathrm{H}, \mathrm{H} 7), 8.00(\mathrm{t}$, $1 \mathrm{H}, \mathrm{H} 6) \mathrm{ppm}$.

dibenzothiophene<smiles>c1ccc2c(c1)sc1ccccc12</smiles>

Figure 111: Molecular structure and numbering of the substrate molecule dibenzothiophene.

${ }^{1} \mathrm{H}$ NMR: $\left(600 \mathrm{MHz}, \mathrm{CD}_{3} \mathrm{OD}\right): \delta=8.26(\mathrm{~m}, 2 \mathrm{H}, \mathrm{H} 1 / \mathrm{H} 9), 7.90(\mathrm{~m}, 2 \mathrm{H}, \mathrm{H} 4 / \mathrm{H} 6), 7.49(\mathrm{~m}, 4 \mathrm{H}$, $\mathrm{H} 2 / \mathrm{H} 3 / \mathrm{H} 7 / \mathrm{H} 8) \mathrm{ppm}$. 


\subsection{Chemical Synthesis}

\subsubsection{Materials}

All solvents were purchased from Merck, Fluka and Acros in pro analysis quality. Chemicals were obtained from Sigma Aldrich, Merck, TCl, Alfa Aesar and Acros and used without further purification. Selected reactions were performed under an argon atmosphere to protect sensitive compounds from exposure to oxygen or moisture.

\subsubsection{Chromatography}

Liquid phase column chromatography was performed for purification using Merck silica gel 60 with particle sizes of either $15-40$ or $63-100 \mu \mathrm{m}$. Crude products were applied as concentrated solutions to the columns holding a 50 to 100 -fold excess of silica. Thin layer chromatography for reaction control was performed using Alugram ${ }^{\circledR}$ SIL G/UV 254 plates from Macherey-Nagel with iodine or phosphomolybdic solution in ethanol (10\%) as optional dyes.

\subsubsection{NMR Spectroscopy}

Synthesized compounds were analyzed using a Bruker Avance I Ultrashielded spectrometer $(400 \mathrm{MHz}$ ) at $298 \mathrm{~K}$. NMR solvents were purchased from Deutero $\mathrm{GmbH}$ with degrees of deuteration of at least $99.8 \%$ and are indicated in the synthesis protocols. Chemical shifts $\delta$ are referenced to residual solvent signals of chloroform $\left(\delta=7.26 \mathrm{ppm}\right.$ for ${ }^{1} \mathrm{H}, \delta=77.0 \mathrm{ppm}$ for ${ }^{13} \mathrm{C}$ ) or dimethyl sulfoxide $\left(\delta=2.50 \mathrm{ppm}\right.$ for ${ }^{1} \mathrm{H}, \delta=39.5$ for $\left.{ }^{13} \mathrm{C}\right)$ and given in parts per million (ppm). Multiplicities are denoted as $s=$ singlet, $d=$ doublet, $t=$ triplet, $m=$ multiplet and $\mathrm{br}=$ broadened. Scalar coupling constants $J$ are given in $\mathrm{Hz}$ and intensities as the relative number of nuclei.

\subsubsection{Mass Spectrometry}

Mass analysis was performed on highly diluted solutions in acetonitrile using a Waters Micromass ZQ electron spray ionization mass spectrometer with a quadrupole detector or Thermo DSQII/Focus GC. Results are given in mass per charge $(\mathrm{m} / \mathrm{z})$. 


\subsubsection{Experimental Details}

Only those reactions that were performed by the author, generated positive results and are not mere reproductions of previously published results are presented in this section.

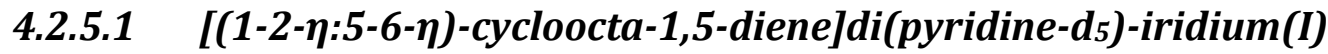 tetrafluoroborate}

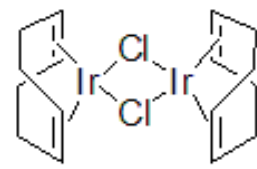

25

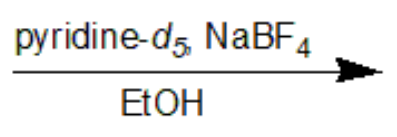

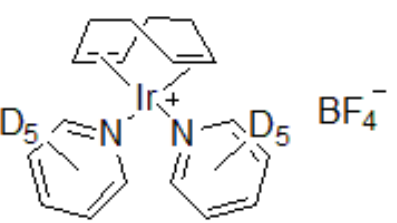

26

671.70

To a solution of bis(1,5-cyclooctadiene)diiridium(I) dichloride (1.00 g, $1.49 \mathrm{mmol}, 1.00 \mathrm{eq}) \mathbf{2 5}$ in ethanol $(200 \mathrm{~mL})$ were added pyridine- $d_{5}(2.40 \mathrm{~mL}, 29.60 \mathrm{mmol}, 20.00 \mathrm{eq})$ and a $1 \mathrm{M}$ aqueous solution of sodium tetrafluoroborate $(50 \mathrm{~mL}, 50 \mathrm{mmol}, 33.56 \mathrm{eq})$ under an argon atmosphere.

The organic solvent was removed under reduced pressure and the precipitate filtered off. The solid was washed with a saturated aqueous solution of sodium tetrafluoroborate and dried under reduced pressure to afford product $26(1.49 \mathrm{~g}, 2.68 \mathrm{mmol}, 90 \%)$ as a bright yellow solid.

${ }^{1} \mathrm{H}$ NMR: $\left(400 \mathrm{MHz}, \mathrm{CDCl}_{3}\right): \delta=3.87-3.80(\mathrm{~m}, 4 \mathrm{H}, \mathrm{COD}-\mathrm{CH}), 2.54-2.41\left(\mathrm{~m}, 4 \mathrm{H}, \mathrm{COD}-\mathrm{CH}_{2}\right)$, $1.88-1.77\left(\mathrm{~m}, 4 \mathrm{H}, \mathrm{COD}-\mathrm{CH}_{2}\right) \mathrm{ppm}$. 


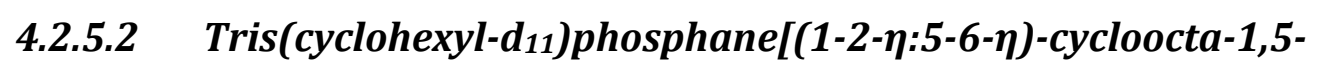
diene](pyridine-d5)-iridium(I) tetrafluoroborate

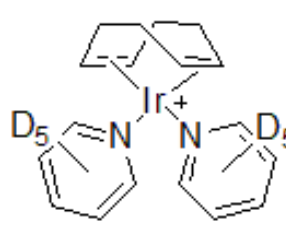

26

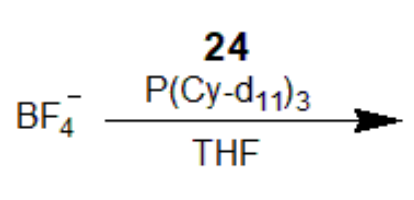

24

555.46

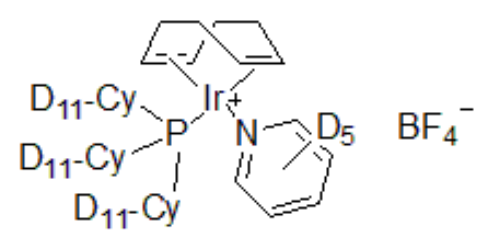

22

A solution of Tri(cyclohexyl- $\left.d_{11}\right)$-phosphane $24(200.70 \mathrm{mg}, 0.64 \mathrm{mmol}, 1.40 \mathrm{eq})$ and [(1-2$\eta: 5-6-\eta)$-cycloocta-1,5-diene]di(pyridine- $d_{5}$ )-iridium(I) tetrafluoroborate $26 \quad(250 \mathrm{mg}$, $0.45 \mathrm{mmol}, 1.00 \mathrm{eq})$ in tetrahydrofuran $(3 \mathrm{~mL}$ ) was stirred at room temperature for $0.5 \mathrm{~h}$.

The precipitate was filtered off and dissolved in dichloromethane $(5 \mathrm{~mL})$. Diethyl ether $(6$ $\mathrm{mL}$ ) was added and the solvent slowly evaporated under an argon stream. The precipitate was filtered off and dried under reduced pressure to afford product $22(283 \mathrm{mg}, 0.36 \mathrm{mmol}$, $81 \%)$ as an orange solid.

${ }^{1} \mathrm{H}$ NMR: $\left(400 \mathrm{MHz}, \mathrm{CDCl}_{3}\right): \delta=4.08-3.99(\mathrm{~m}, 4 \mathrm{H}, \mathrm{COD}-\mathrm{CH}), 2.45-2.22\left(\mathrm{~m}, 4 \mathrm{H}, \mathrm{COD}-\mathrm{CH}_{2}\right)$, $1.78-1.65\left(\mathrm{~m}, 4 \mathrm{H}, \mathrm{COD}-\mathrm{CH}_{2}\right) \mathrm{ppm}$. 


\subsubsection{N,N-dibenzyl-(4-bromo-2,6-dimethylanilin)}<smiles>Cc1cc(Br)cc(C)c1N</smiles>

2

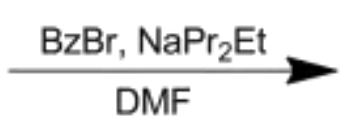

200.08<smiles>Cc1cc(Br)cc(C)c1N(C(=O)c1ccccc1)C(=O)c1ccccc1</smiles>

3

380.32

Benzyl bromide $(1.80 \mathrm{~mL}, 15.0 \mathrm{mmol}, 3.00 \mathrm{eq})$ and ethyldiisopropylamine $(2.61 \mathrm{~mL}, 15.0$ $\mathrm{mmol}, 3.00 \mathrm{eq})$ were added to a solution of 4-bromo-2,6-dimethylanilin $(1.01 \mathrm{~g}, 5 \mathrm{mmol}$, $1.00 \mathrm{eq})$ in DMF $(8 \mathrm{~mL})$. The reaction mixture was heated to reflux for $4 \mathrm{~h}$.

The solvent was removed under reduced pressure and the residue was taken up in $\mathrm{H}_{2} \mathrm{O}$ $(10 \mathrm{~mL})$. The aqueous solution was extracted with ethyl acetate and the organic phase dried over $\mathrm{Na}_{2} \mathrm{SO}_{4}$. The crude product was purified by chromatography on silica using dichloromethane to yield product 3 (1.88 $\mathrm{g}, 4.94 \mathrm{mmol}, 99 \%)$ as a colorless oil.

${ }^{1} \mathrm{H}$ NMR: $\left(400 \mathrm{MHz}, \mathrm{CDCl}_{3}\right): \delta=7.31-7.23(\mathrm{~m}, 6 \mathrm{H}, \mathrm{Ph}), 7.22-7.17(\mathrm{~m}, 4 \mathrm{H}, \mathrm{Ph}), 7.19(\mathrm{~s}, 2 \mathrm{H}, \mathrm{Ph})$, $4.07\left(\mathrm{~s}, 4 \mathrm{H}, \mathrm{CH}_{2}\right), 2.11\left(\mathrm{~s}, 6 \mathrm{H}, \mathrm{CH}_{3}\right) \mathrm{ppm}$.

ESI-MS $\mathrm{m} / \mathrm{z}$ : (acetonitrile, positive mode): calc. for $\mathrm{C}_{22} \mathrm{H}_{23} \mathrm{BrN}[\mathrm{M}+\mathrm{H}]^{+}:$380.10; found 380.07, 382.07. 


\subsubsection{4-(N,N-dibenzylamino)-3,5-dimethylphenylboronic acid}<smiles>Cc1cc(Br)cc(C)c1N(C(=O)c1ccccc1)C(=O)c1ccccc1</smiles>

3

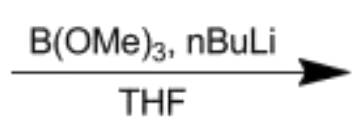

\subsection{2}

4<smiles>Cc1cc([Se]O)cc(C)c1N(C(=O)O)C(=O)c1ccccc1</smiles>

345.24

A $2.5 \mathrm{M} \mathrm{n}$-butyllithium solution in hexane $(1.41 \mathrm{~mL}, 3.53 \mathrm{mmol}, 1.13 \mathrm{eq})$ was added dropwise to a solution of $\mathrm{N}, \mathrm{N}$-dibenzyl-(4-bromo-2,6-dimethylanilin) 3 (1.19 g, $3.13 \mathrm{mmol}, 1.00 \mathrm{eq})$ in dry tetrahydrofuran $(6 \mathrm{~mL})$ under an argon atmosphere at $-78^{\circ} \mathrm{C}$. The solution was stirred for $2 \mathrm{~h}$ and trimethyl borate $(1.04 \mathrm{~mL}, 9.25 \mathrm{mmol}, 2.96 \mathrm{eq})$ was added. The reaction mixture was allowed to warm to room temperature overnight and the reaction was quenched by addition of $1 \mathrm{~N}$ hydrochloric acid $(15 \mathrm{~mL})$.

The solution was extracted using diethyl ether and dried over sodium sulfate. The solvent was evaporated under reduced pressure and the crude product washed with petroleum ether to give product 4 (754 mg, $2.18 \mathrm{mmol}, 70 \%$ ) as a blunt, white solid.

${ }^{1} \mathrm{H}$ NMR: (400 MHz, DMSO): $\delta=7.81(\mathrm{~s}, 2 \mathrm{H}, \mathrm{OH}), 7.38-7.15(\mathrm{~m}, 12 \mathrm{H}, \mathrm{Ph}), 4.07\left(\mathrm{~s}, 4 \mathrm{H} \mathrm{CH} \mathrm{CH}_{2}\right)$, $2.12\left(\mathrm{~s}, 6 \mathrm{H}, \mathrm{CH}_{3}\right) \mathrm{ppm}$. 


\subsubsection{Methyl 4-(4'-(N,N-dibenzylamino)-3',5'-dimethylphenylmethyl)benzoate}<smiles>Cc1cc([Hg]O)cc(C)c1N(C(=O)c1ccccc1)C(=O)c1ccccc1</smiles>

4

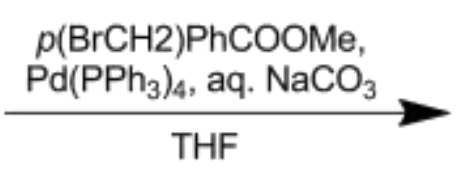

THF<smiles>COC(=O)c1ccc(Cc2cc(C)c(N(C(=O)c3ccccc3)C(=O)c3ccccc3)c(C)c2)cc1</smiles>

5

345.24

449.58

To a solution of 4-(N,N-dibenzylamino)-3,5-dimethylphenylboronic acid 4 (3.53 g, $10.2 \mathrm{mmol}$, $1 \mathrm{eq})$ in tetrahydrofuran $(60 \mathrm{~mL})$ were added methyl 4-bromomethylbenzoate $(2.23 \mathrm{~g}$, $9.74 \mathrm{mmol}, 0.95 \mathrm{eq}), 1.2 \mathrm{M}$ aqueous sodium carbonate solution $(15.8 \mathrm{~mL}, 19.0 \mathrm{mmol}$, $1.86 \mathrm{eq}$ ) and Tetrakis(triphenylphosphane)palladium(0) (106 mg, $0.09 \mathrm{mmol}, 0.90 \mathrm{~mol} \%)$ under an argon atmosphere. The reaction was heated to reflux for $3 \mathrm{~h}$ and stirred at $0{ }^{\circ} \mathrm{C}$ for $0.5 \mathrm{~h}$.

The organic phase was separated and filtered through Celite. The solvent was removed under reduced pressure and the crude product purified by chromatography on silica using hexane/ethyl acetate (95:5) to afford product 5 (3.30 g, $7.34 \mathrm{mmol}, 75 \%)$ as a colorless oil.

${ }^{1} \mathrm{H}$ NMR: (400 MHz, $\mathrm{CDCl}_{3}$ ): $\delta=7.98$ (dt, 2H, $\left.\underline{\mathrm{Ph}}-\mathrm{COOMe}\right), 7.33-7.12(\mathrm{~m}, 12 \mathrm{H}, \mathrm{Ph}), 6.78(\mathrm{~s}, 2 \mathrm{H}$, $\mathrm{Me}_{2}-\underline{\mathrm{Ph}}$ ), 4.09 (s, 4H, N-CH ), 3.92 (s, 2H, Ph- $\mathrm{CH}_{2}-\mathrm{Ph}$ ), 3.91 (s, 3H, COOMe), 2.13 (s, 6H, PhMe) ppm.

ESI-MS m/z: (acetonitrile, positive mode): calc. for $\mathrm{C}_{31} \mathrm{H}_{32} \mathrm{BrN}[\mathrm{M}+\mathrm{H}]^{+}:$450.24; found 450.30 . 


\subsubsection{Methyl 4-(4'-amino-3',5'-dimethylphenylmethyl)benzoate}<smiles>COC(=O)c1ccc(Cc2cc(C)c(N(Cc3ccccc3)Cc3ccccc3)c(C)c2)cc1</smiles>

5

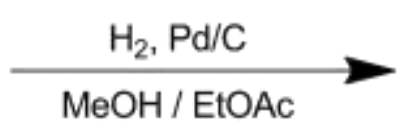<smiles>COC(=O)c1ccc(Cc2cc(C)c(N)c(C)c2)cc1</smiles>

6

449.58

269.34

Methyl 4-(4'-(N,N-dibenzylamino)-3',5'-dimethylphenylmethyl)benzoate $\quad \mathbf{5} \quad(3.30 \mathrm{~g}$, $7.34 \mathrm{mmol}, 1 \mathrm{eq}$ ) and $10 \mathrm{wt} . \%$ palladium supported on carbon $(500 \mathrm{mg}, 0.47 \mathrm{mmol}, 6.40$ mol\%) were suspended in methanol/ethyl acetate (1:1) $(150 \mathrm{~mL})$ under an argon atmosphere. Hydrogen gas was passed into the solution for $4 \mathrm{~h}$ under stirring.

The reaction mixture was filtered through Celite and the solvent removed under reduced pressure to afford product $6(1.96 \mathrm{~g}, 7.26 \mathrm{mmol}, 99 \%)$ as an off-white solid.

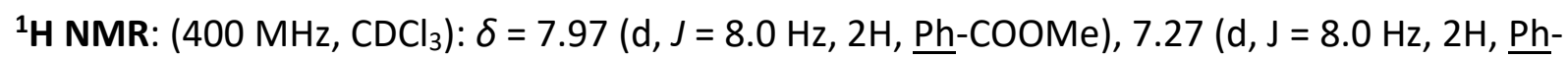
$\left.\mathrm{CH}_{2}-\mathrm{Ph}\right), 6.78(\mathrm{~s}, 2 \mathrm{H}, \mathrm{Ph}), 3.91(\mathrm{~s}, 3 \mathrm{H}, \mathrm{COOMe}), 3.90\left(\mathrm{~s}, 2 \mathrm{H}, \mathrm{CH}_{2}\right), 3.41\left(\mathrm{sbr}, 2 \mathrm{H}, \mathrm{NH}_{2}\right), 2.16(\mathrm{~s}$, $6 \mathrm{H}, \mathrm{Ph}-\underline{\mathrm{Me}}) \mathrm{ppm}$.

ESI-MS $m / z$ : (acetonitrile, positive mode): calc. for $\mathrm{C}_{17} \mathrm{H}_{20} \mathrm{NO}_{2}[\mathrm{M}+\mathrm{H}]^{+}: 270.15$; found 270.20 . 


\subsubsection{N-(2,4,6-trimethylphenyl)-N'-(4-(4-(methylcarboxy)phenylmethyl)-2,6- dimethylphenyl)formamidine}<smiles>COC(=O)c1ccc(Cc2cc(C)c(N)c(C)c2)cc1</smiles>

6

269.34 2,4,6-trimethylanilin $\mathrm{HC}(\mathrm{OEt})_{3}, \mathrm{CH}_{3} \mathrm{COOH}$

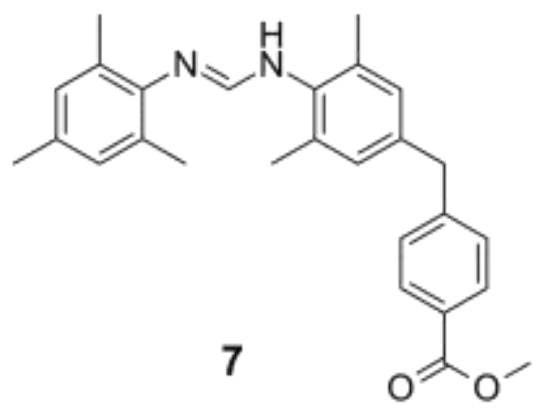

414.54

A mixture of methyl 4-(4'-amino-3', 5'-dimethylphenylmethyl)benzoate 6 (1.30 g, $4.86 \mathrm{mmol}$, $1.00 \mathrm{eq})$, triethyl orthoformate $(0.68 \mathrm{~mL}, 4.68 \mathrm{mmol}, 1.00 \mathrm{eq})$ and acetic acid $(15.0 \mu \mathrm{L}$, $0.26 \mathrm{mmol}, 0.05 \mathrm{eq})$ was heated to $140{ }^{\circ} \mathrm{C}$ for $2 \mathrm{~h} .2,4,6$-Trimethylanilin $(0.72 \mathrm{~g}, 4.86 \mathrm{mmol}$, $1.00 \mathrm{eq}$ ) was added and the reaction mixture stirred at $140{ }^{\circ} \mathrm{C}$ overnight.

The solidified mixture was triturated using hexane and filtered. The crude product was purified by chromatography on silica using hexane/dichloromethane/triethylamine $(8: 1: 1)$ to afford product 7 (1.20 g, $2.90 \mathrm{mmol}, 60 \%)$ as a white solid.

${ }^{1} \mathrm{H}$ NMR: (400 MHz, DMSO): $\delta=8.06\left(\mathrm{~s}_{\mathrm{br}}, 1 \mathrm{H}, \mathrm{NH}\right), 7.87(\mathrm{~d}, 2 \mathrm{H}, \mathrm{Ph}-\mathrm{H}), 7.36\left(\mathrm{~d}_{\mathrm{br}}, 2 \mathrm{H}, \mathrm{Ph}-\mathrm{H}\right)$, 6.99-6.64 (m, 4H, Ph-H), $3.87\left(\mathrm{sbr}, 2 \mathrm{H}, \mathrm{Ph}-\underline{\mathrm{CH}_{2}}-\mathrm{Ph}\right), 3.80(\mathrm{~s}, 3 \mathrm{H}, \mathrm{COOMe}), 2.40-1.80(\mathrm{~m}, 15 \mathrm{H}$, $\left.\mathrm{CH}_{3}\right) \mathrm{ppm}$.

ESI-MS m/z: (acetonitrile, positive mode): calc. for $\mathrm{C}_{27} \mathrm{H}_{31} \mathrm{~N}_{2} \mathrm{O}_{2}[\mathrm{M}+\mathrm{H}]^{+}: 415.24$; found 415.30 . 


\subsubsection{1-(2,4,6-trimethylphenyl)-3-(4-(4-(methylcarboxy)phenylmethyl)-2,6-} dimethylphenyl)-4-hydroxy-4,5-dihydroimidazolium chloride<smiles>COC(=O)c1ccc(Cc2cc(C)c(N/C=N/c3c(C)cc(C)cc3C)c(C)c2)cc1</smiles>

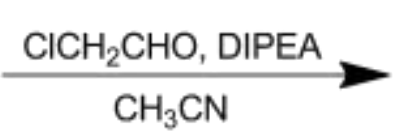

To a solution of $\mathrm{N}$-(2,4,6-trimethylphenyl)-N'-(4-(4-(methylcarboxy)phenylmethyl)-2,6dimethylphenyl)formamidine $\quad 7 \quad(100 \mathrm{mg}, \quad 0.24 \mathrm{mmol}, \quad 1.00 \mathrm{eq})$ were added chloroacetaldehyde $(160 \mu \mathrm{L}, 0.96 \mathrm{mmol}, 4.00 \mathrm{eq})$ and ethyldiisopropylamine $(95.0 \mu \mathrm{L}$, $0.56 \mathrm{mmol}, 2.33 \mathrm{eq}$ ) and the resulting mixture was stirred at $120^{\circ} \mathrm{C}$ for $0.5 \mathrm{~h}$.

The solvent was removed under reduced pressure and the crude product purified by chromatography on silica using dichloromethane/methanol (20:1) to afford product 8 (65.1 $\mathrm{mg}, 0.13 \mathrm{mmol}, 55 \%)$ as a white solid.

${ }^{1} \mathrm{H}$ NMR: (400 MHz, MeOD): $\delta=8.55(\mathrm{~s}, 1 \mathrm{H}, \mathrm{N}-\underline{\mathrm{H}}=\mathrm{N}), 7.92(\mathrm{~m}, 2 \mathrm{H}, \mathrm{Ph}-\mathrm{H}), 7.40-6.74(\mathrm{~m}, 6 \mathrm{H}$, $\mathrm{Ph}-\mathrm{H}), 5.49\left(2 \mathrm{H}, \mathrm{Ph}-\underline{\mathrm{C}}_{2}-\mathrm{Ph}\right), 4.58\left(\mathrm{~d} \mathrm{br}, 1 \mathrm{H}, \mathrm{N}-\underline{\mathrm{C}}_{2}\right), 4.34(\mathrm{~m}, 1 \mathrm{H}, \underline{\mathrm{C}}-\mathrm{OH}), 4.03(\mathrm{~d} b r, 1 \mathrm{H} . \mathrm{N}-\underline{\mathrm{CH}} 2)$, $3.88(\mathrm{~m}, 3 \mathrm{H}, \mathrm{COOMe}), 2.49-1.93(\mathrm{~m}, 15 \mathrm{H}, \mathrm{Ph}-\underline{\mathrm{Me}}) \mathrm{ppm}$.

ESI-MS $m / z$ : (acetonitrile, positive mode): calc. for $\mathrm{C}_{29} \mathrm{H}_{33} \mathrm{~N}_{2} \mathrm{O}_{3}[\mathrm{M}]^{+}:$: 457.25; found 457.30. 


\subsubsection{9 [4-(Bromomethyl)phenoxymethyl]polystyrene}

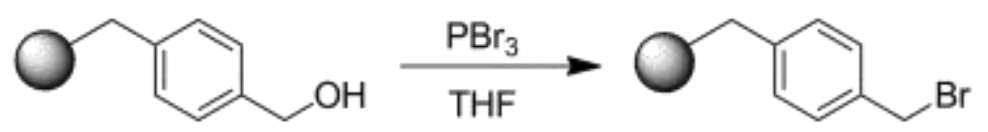

13

14

To a suspension of [4-(Hydroxymethyl)phenoxymethyl]polystyrene 13 (1.00 g, $0.5-1.2$ $\mathrm{mmol} \mathrm{OH}$ functionality, $1.00 \mathrm{eq})$ in tetrahydrofuran $(40 \mathrm{~mL})$ at $-20^{\circ} \mathrm{C}$ phosphorus tribromide $(0.15 \mathrm{~mL}, 1.60 \mathrm{mmol}, 1.33-3.20 \mathrm{eq})$ was added and the reaction mixture was shaken for $12 \mathrm{~h}$.

The solid particles were filtered, washed with aq. $\mathrm{NaHCO}_{3}$ and continuously extracted with diethyl ether for $48 \mathrm{~h}$. The polymer beads were dried under removed pressure to afford functionalized polymer $\mathbf{1 4}$ as an off-yellow solid.

${ }^{1} \mathrm{H} \mathrm{NMR}^{*}:\left(400 \mathrm{MHz}, \mathrm{CD}_{2} \mathrm{Cl}_{2}\right): \delta=4.56\left(\mathrm{sbr}, 2 \mathrm{H}, \mathrm{CH}_{2} \mathrm{Br}\right) \mathrm{ppm}$.

*Note: Due to the polymeric nature of the carrier compound, all resonances appear severely broadened and some are buried under the polymer resonance, so that not all resonances could be deconvoluted and assigned. 


\subsubsection{0 [4-(((2,4,6-trimethylphenyl)imidazoline)methyl)phenoxymethyl]- polystyrene bromide}

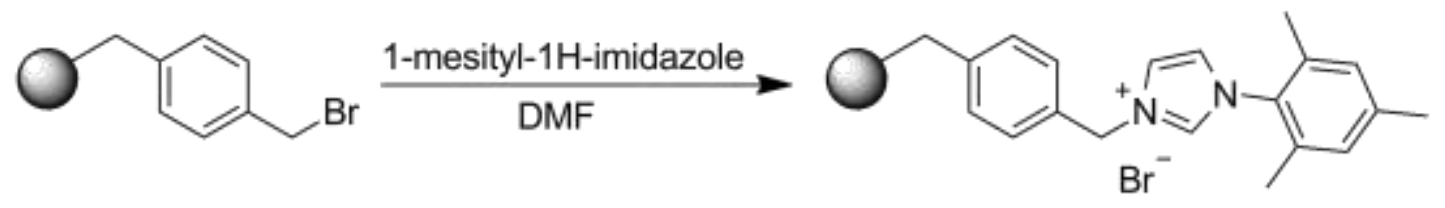

14

16

[4-(Bromomethyl)phenoxymethyl]polystyrene 14 (1.00 g, $0.50-1.20 \mathrm{mmol} \mathrm{Br}$ functionality, $1.00 \mathrm{eq})$ and 1-mesityl- $1 \mathrm{H}$-imidazole $(280 \mathrm{mg}, 1.5 \mathrm{mmol}, 1.25-3.00 \mathrm{eq})$ were placed in a round-bottom flask under an argon atmosphere. Dry dimethylformamide $(30 \mathrm{~mL})$ was added and the reaction mixture shaken at $80^{\circ} \mathrm{C}$ for $16 \mathrm{~h}$.

The solid particles were filtered, washed with diethyl ether and continuously extracted with methanol for $48 \mathrm{~h}$. The polymer beads were dried under removed pressure to afford functionalized polymer $\mathbf{1 6}$ as a brownish solid.

${ }^{1} \mathrm{H} \mathrm{NMR}^{*}:\left(400 \mathrm{MHz}, \mathrm{CD}_{2} \mathrm{Cl}_{2}\right): \delta=7.42-6.78\left(\mathrm{~m}_{\mathrm{br}}, 6 \mathrm{H}, \mathrm{Ph}-\mathrm{H}\right) 2.37$ ( $\mathrm{s}_{\mathrm{br}}, 3 \mathrm{H}, \mathrm{p}$-mesityl-Me), 2.09 (sbr, 6H, o-mesityl-Me) ppm.

*Note: Due to the polymeric nature of the carrier compound, all resonances appear severely broadened and some are buried under the polymer backbone resonance, so that not all resonances could be deconvoluted and assigned. 


\section{Bibliography}

[1] G.A. Morris, R. Freeman, Enhancement of nuclear magnetic resonance signals by polarization transfer, J Am Chem Soc, 101 (1979) 760-762.

[2] G.A. Morris, Sensitivity enhancement in nitrogen-15 NMR: polarization transfer using the INEPT pulse sequence, J Am Chem Soc, 102 (1980) 428-429.

[3] K.H. Gardner, L.E. Kay, THE USE OF 2H, 13C, 15N MULTIDIMENSIONAL NMR GTO STUDY THE STRUCTURE AND DYNAMICS OF PROTEINS, Annual Review of Biophysics and Biomolecular Structure, 27 (1998) 357-406.

[4] N.D. Bhaskar, W. Happer, T. McClelland, Efficiency of Spin Exchange between Rubidium Spins and ${ }^{129}$ Xe Nuclei in a Gas, Physical Review Letters, 49 (1982) 25-28.

[5] A. Abragam, M. Goldman, Principles of Dynamic Nuclear-Polarization, Rep Prog Phys, 41 (1978) 395-467.

[6] K.H. Hausser, D. Stehlik, Dynamic Nuclear Polarization in Liquids, in: W. John S (Ed.) Advances in Magnetic and Optical Resonance, Academic Press, 1968, pp. 79-139.

[7] C.R. Bowers, D.P. Weitekamp, Transformation of symmetrization order to nuclear-spin magnetization by chemical reaction and nuclear magnetic resonance, Phys Rev Lett, 57 (1986) 26452648.

[8] F.D. Colegrove, L.D. Schearer, G.K. Walters, Polarization of $\mathrm{He}^{3}$ Gas by Optical Pumping, Physical Review, 132 (1963) 2561-2572.

[9] B.M. Goodson, Nuclear Magnetic Resonance of Laser-Polarized Noble Gases in Molecules, Materials, and Organisms, J Magn Reson, 155 (2002) 157-216.

[10] M. Reese, M.-T. Türke, I. Tkach, G. Parigi, C. Luchinat, T. Marquardsen, A. Tavernier, P. Höfer, F. Engelke, C. Griesinger, M. Bennati, $1 \mathrm{H}$ and $13 \mathrm{C}$ Dynamic Nuclear Polarization in Aqueous Solution with a Two-Field (0.35 T/14 T) Shuttle DNP Spectrometer, J Am Chem Soc, 131 (2009) 15086-15087.

[11] A. Krahn, P. Lottmann, T. Marquardsen, A. Tavernier, M.-T. Turke, M. Reese, A. Leonov, M. Bennati, P. Hoefer, F. Engelke, C. Griesinger, Shuttle DNP spectrometer with a two-center magnet, Phys Chem Chem Phys, 12 (2010) 5830-5840.

[12] M. Marjańska, I. Iltis, A.A. Shestov, D.K. Deelchand, C. Nelson, K. Uğurbil, P.-G. Henry, In vivo $13 C$ spectroscopy in the rat brain using hyperpolarized [1-13C]pyruvate and [2-13C]pyruvate, J Magn Reson, 206 (2010) 210-218.

[13] U.L. Günther, Dynamic Nuclear Hyperpolarization in Liquids, in: H. Heise, S. Matthews (Eds.) Modern NMR Methodology, Springer Berlin Heidelberg, Berlin, Heidelberg, 2013, pp. 23-69.

[14] T. Maly, G.T. Debelouchina, V.S. Bajaj, K.-N. Hu, C.-G. Joo, M.L. Mak-Jurkauskas, J.R. Sirigiri, P.C.A. van der Wel, J. Herzfeld, R.J. Temkin, R.G. Griffin, Dynamic nuclear polarization at high magnetic fields, The Journal of chemical physics, 128 (2008) 052211-052211.

[15] J. Natterer, J. Bargon, Parahydrogen induced polarization, Prog Nucl Mag Res Sp, 31 (1997) 293315.

[16] A.B. Permin, R. Eisenberg, One-Hydrogen Polarization in Hydroformylation Promoted by Platinum-Tin and Iridium Carbonyl Complexes: A New Type of Parahydrogen-Induced Effect, J Am Chem Soc, 124 (2002) 12406-12407.

[17] S.B. Duckett, C.J. Sleigh, Applications of the parahydrogen phenomenon: A chemical perspective, Prog Nucl Mag Res Sp, 34 (1999) 71-92.

[18] Simon B. Duckett, D. Blazina, The Study of Inorganic Systems by NMR Spectroscopy in Conjunction with Parahydrogen-Induced Polarisation, European Journal of Inorganic Chemistry, 2003 (2003) 2901-2912. 
[19] D. Blazina, S.B. Duckett, J.P. Dunne, C. Godard, Applications of the parahydrogen phenomenon in inorganic chemistry, Dalton transactions, (2004) 2601-2609.

[20] R.A. Green, R.W. Adams, S.B. Duckett, R.E. Mewis, D.C. Williamson, G.G.R. Green, The theory and practice of hyperpolarization in magnetic resonance using parahydrogen, Prog Nucl Mag Res Sp, 67 (2012) 1-48.

[21] K.D. Atkinson, M.J. Cowley, S.B. Duckett, P.I.P. Elliott, G.G.R. Green, J. López-Serrano, I.G. Khazal, A.C. Whitwood, Para-Hydrogen Induced Polarization without Incorporation of Para-Hydrogen into the Analyte, Inorganic chemistry, 48 (2009) 663-670.

[22] R.W. Adams, J.A. Aguilar, K.D. Atkinson, M.J. Cowley, P.I.P. Elliott, S.B. Duckett, G.G.R. Green, I.G. Khazal, J. López-Serrano, D.C. Williamson, Reversible Interactions with para-Hydrogen Enhance NMR Sensitivity by Polarization Transfer, Science, 323 (2009) 1708-1711.

[23] R.H. Crabtree, H. Felkin, T. Fillebeen-Khan, G.E. Morris, Dihydridoiridium diolefin complexes as intermediates in homogeneous hydrogenation, Journal of Organometallic Chemistry, 168 (1979) 183195.

[24] R.H. Crabtree, H. Felkin, G.E. Morris, Cationic iridium diolefin complexes as alkene hydrogenation catalysts and the isolation of some related hydrido complexes, Journal of Organometallic Chemistry, 141 (1977) 205-215.

[25] K.D. Atkinson, M.J. Cowley, P.I.P. Elliott, S.B. Duckett, G.G.R. Green, J. López-Serrano, A.C. Whitwood, Spontaneous Transfer of Parahydrogen Derived Spin Order to Pyridine at Low Magnetic Field, J Am Chem Soc, 131 (2009) 13362-13368.

[26] R.W. Adams, S.B. Duckett, R.A. Green, D.C. Williamson, G.G.R. Green, A theoretical basis for spontaneous polarization transfer in non-hydrogenative parahydrogen-induced polarization, The Journal of Chemical Physics, 131 (2009) 194505.

[27] M. Fekete, O.W. Bayfield, S.B. Duckett, S. Hart, R.E. Mewis, N. Pridmore, P.J. Rayner, A. Whitwood, Iridium(III) hydrido N-heterocyclic carbene-phosphine complexes as catalysts in magnetization transfer reactions, Inorganic chemistry, 52 (2013) 13453-13461.

[28] J.B. Hövener, N. Schwaderlapp, R. Borowiak, T. Lickert, S.B. Duckett, R.E. Mewis, R.W. Adams, M.J. Burns, L.A.R. Highton, G.G.R. Green, A. Olaru, J. Hennig, D. von Elverfeldtt, Toward Biocompatible Nuclear Hyperpolarization Using Signal Amplification by Reversible Exchange: Quantitative in Situ Spectroscopy and High-Field Imaging, Anal Chem, 86 (2014) 1767-1774.

[29] R.E. Mewis, K.D. Atkinson, M.J. Cowley, S.B. Duckett, G.G. Green, R.A. Green, L.A. Highton, D. Kilgour, L.S. Lloyd, J.A. Lohman, D.C. Williamson, Probing signal amplification by reversible exchange using an NMR flow system, Magnetic resonance in chemistry : MRC, 52 (2014) 358-369.

[30] H. Zeng, J. Xu, M.T. McMahon, J.A. Lohman, P.C. van Zijl, Achieving 1\% NMR polarization in water in less than 1min using SABRE, J Magn Reson, 246 (2014) 119-121.

[31] K.M. Appleby, R.E. Mewis, A.M. Olaru, G.G.R. Green, I.J.S. Fairlamb, S.B. Duckett, Investigating pyridazine and phthalazine exchange in a series of iridium complexes in order to define their role in the catalytic transfer of magnetisation from para-hydrogen, Chem Sci, 6 (2015) 3981-3993.

[32] M. Fekete, C. Gibard, G.J. Dear, G.G. Green, A.J. Hooper, A.D. Roberts, F. Cisnetti, S.B. Duckett, Utilisation of water soluble iridium catalysts for signal amplification by reversible exchange, Dalton transactions, 44 (2015) 7870-7880.

[33] A.J. Ruddlesden, R.E. Mewis, G.G.R. Green, A.C. Whitwood, S.B. Duckett, Catalytic Transfer of Magnetism Using a Neutral Iridium Phenoxide Complex, Organometallics, 34 (2015) 2997-3006.

[34] M.J. Cowley, R.W. Adams, K.D. Atkinson, M.C. Cockett, S.B. Duckett, G.G. Green, J.A. Lohman, R. Kerssebaum, D. Kilgour, R.E. Mewis, Iridium N-heterocyclic carbene complexes as efficient catalysts for magnetization transfer from para-hydrogen, J Am Chem Soc, 133 (2011) 6134-6137.

[35] E.B. Dücker, L.T. Kuhn, K. Münnemann, C. Griesinger, Similarity of SABRE field dependence in chemically different substrates, J Magn Reson, 214 (2012) 159-165. 
[36] S. Grosse, A.V. Yurkovskaya, J. Lopez, H.M. Vieth, Field dependence of Chemically Induced Dynamic Nuclear Polarization (CIDNP) in the photoreaction of $\mathrm{N}$-acetyl histidine with 2,2'-dipyridyl in aqueous solution, Journal of Physical Chemistry A, 105 (2001) 6311-6319.

[37] A.N. Pravdivtsev, A.V. Yurkovskaya, H.M. Vieth, K.L. Ivanov, R. Kaptein, Level Anti-Crossings are a Key Factor for Understanding para-Hydrogen-Induced Hyperpolarization in SABRE Experiments, Chemphyschem, 14 (2013) 3327-3331.

[38] R.E. Mewis, Developments and advances concerning the hyperpolarisation technique SABRE, Magnetic resonance in chemistry : MRC, 53 (2015) 789-800.

[39] J.A. Aguilar, P.I.P. Elliott, J. Lopez-Serrano, R.W. Adams, S.B. Duckett, Only para-hydrogen spectroscopy (OPSY), a technique for the selective observation of para-hydrogen enhanced NMR signals, Chem Commun, (2007) 1183-1185.

[40] B.J. van Weerdenburg, S. Glöggler, N. Eshuis, A.H. Engwerda, J.M. Smits, R. de Gelder, S. Appelt, S.S. Wymenga, M. Tessari, M.C. Feiters, B. Blümich, F.P. Rutjes, Ligand effects of NHC-iridium catalysts for signal amplification by reversible exchange (SABRE), Chem Commun (Camb), 49 (2013) 7388-7390.

[41] R.A. Kelly III, H. Clavier, S. Giudice, N.M. Scott, E.D. Stevens, J. Bordner, I. Samardjiev, C.D. Hoff, L. Cavallo, S.P. Nolan, Determination of N-Heterocyclic Carbene (NHC) Steric and Electronic Parameters using the [(NHC)Ir(CO)2Cl] System, Organometallics, 27 (2008) 202-210.

[42] H. Clavier, S.P. Nolan, Percent buried volume for phosphine and $\mathrm{N}$-heterocyclic carbene ligands: steric properties in organometallic chemistry, Chem Commun, 46 (2010) 841-861.

[43] S.V.C. Vummaleti, D.J. Nelson, A. Poater, A. Gomez-Suarez, D.B. Cordes, A.M.Z. Slawin, S.P. Nolan, L. Cavallo, What can NMR spectroscopy of selenoureas and phosphinidenes teach us about the pi-accepting abilities of N-heterocyclic carbenes?, Chem Sci, 6 (2015) 1895-1904.

[44] B.J. van Weerdenburg, N. Eshuis, M. Tessari, F.P. Rutjes, M.C. Feiters, Application of the piaccepting ability parameter of $\mathrm{N}$-heterocyclic carbene ligands in iridium complexes for signal amplification by reversible exchange (SABRE), Dalton transactions, 44 (2015) 15387-15390.

[45] L.S. Lloyd, A. Asghar, M.J. Burns, A. Charlton, S. Coombes, M.J. Cowley, G.J. Dear, S.B. Duckett, G.R. Genov, G.G.R. Green, L.A.R. Highton, A.J.J. Hooper, M. Khan, I.G. Khazal, R.J. Lewis, R.E. Mewis, A.D. Roberts, A.J. Ruddlesden, Hyperpolarisation through reversible interactions with parahydrogen, Catalysis Science \& Technology, 4 (2014) 3544-3554.

[46] A.J. Holmes, P.J. Rayner, M.J. Cowley, G.G. Green, A.C. Whitwood, S.B. Duckett, The reaction of an iridium PNP complex with parahydrogen facilitates polarisation transfer without chemical change, Dalton transactions, 44 (2015) 1077-1083.

[47] L.S. Lloyd, R.W. Adams, M. Bernstein, S. Coombes, S.B. Duckett, G.G.R. Green, R.J. Lewis, R.E. Mewis, C.J. Sleigh, Utilization of SABRE-Derived Hyperpolarization To Detect Low-Concentration Analytes via 1D and 2D NMR Methods, J Am Chem Soc, 134 (2012) 12904-12907.

[48] L. Frydman, T. Scherf, A. Lupulescu, The acquisition of multidimensional NMR spectra within a single scan, Proceedings of the National Academy of Sciences, 99 (2002) 15858-15862.

[49] H. Zeng, J. Xu, J. Gillen, M.T. McMahon, D. Artemov, J.M. Tyburn, J.A. Lohman, R.E. Mewis, K.D. Atkinson, G.G. Green, S.B. Duckett, P.C. van Zijl, Optimization of SABRE for polarization of the tuberculosis drugs pyrazinamide and isoniazid, J Magn Reson, 237 (2013) 73-78.

[50] R.E. Mewis, M. Fekete, G.G.R. Green, A.C. Whitwood, S.B. Duckett, Deactivation of signal amplification by reversible exchange catalysis, progress towards in vivo application, Chem Commun, 51 (2015) 9857-9859.

[51] N. Eshuis, N. Hermkens, B.J.A. van Weerdenburg, M.C. Feiters, F.P.J.T. Rutjes, S.S. Wijmenga, M. Tessari, Toward Nanomolar Detection by NMR Through SABRE Hyperpolarization, J Am Chem Soc, 136 (2014) 2695-2698. 
[52] N. Eshuis, B.J. van Weerdenburg, M.C. Feiters, F.P. Rutjes, S.S. Wijmenga, M. Tessari, Quantitative trace analysis of complex mixtures using SABRE hyperpolarization, Angewandte Chemie, 54 (2015) 1481-1484.

[53] N. Eshuis, R.L. Aspers, B.J. van Weerdenburg, M.C. Feiters, F.P. Rutjes, S.S. Wijmenga, M. Tessari, 2D NMR Trace Analysis by Continuous Hyperpolarization at High Magnetic Field, Angewandte Chemie, 54 (2015) 14527-14530.

[54] N.K. Hermkens, N. Eshuis, B.J. van Weerdenburg, M.C. Feiters, F.P. Rutjes, S.S. Wijmenga, M. Tessari, NMR-Based Chemosensing via p-H2 Hyperpolarization: Application to Natural Extracts, Anal Chem, 88 (2016) 3406-3412.

[55] V. Daniele, F.X. Legrand, P. Berthault, J.N. Dumez, G. Huber, Single-Scan Multidimensional NMR Analysis of Mixtures at Sub-Millimolar Concentrations by using SABRE Hyperpolarization, Chemphyschem, 16 (2015) 3413-3417.

[56] R.E. Mewis, R.A. Green, M.C. Cockett, M.J. Cowley, S.B. Duckett, G.G. Green, R.O. John, P.J. Rayner, D.C. Williamson, Strategies for the hyperpolarization of acetonitrile and related ligands by SABRE, J Phys Chem B, 119 (2015) 1416-1424.

[57] K.X. Moreno, K. Nasr, M. Milne, A.D. Sherry, W.J. Goux, Nuclear spin hyperpolarization of the solvent using signal amplification by reversible exchange (SABRE), J Magn Reson, 257 (2015) 15-23.

[58] V.V. Zhivonitko, I.V. Skovpin, I.V. Koptyug, Strong 31P nuclear spin hyperpolarization produced via reversible chemical interaction with parahydrogen, Chem Commun (Camb), 51 (2015) 2506-2509.

[59] M.J. Burns, P.J. Rayner, G.G.R. Green, L.A.R. Highton, R.E. Mewis, S.B. Duckett, Improving the Hyperpolarization of P-31 Nuclei by Synthetic Design, J Phys Chem B, 119 (2015) 5020-5027.

[60] T. Theis, M.L. Truong, A.M. Coffey, R.V. Shchepin, K.W. Waddell, F. Shi, B.M. Goodson, W.S. Warren, E.Y. Chekmenev, Microtesla SABRE Enables 10\% Nitrogen-15 Nuclear Spin Polarization, J Am Chem Soc, 137 (2015) 1404-1407.

[61] R.V. Shchepin, M.L. Truong, T. Theis, A.M. Coffey, F. Shi, K.W. Waddell, W.S. Warren, B.M. Goodson, E.Y. Chekmenev, Hyperpolarization of "Neat" Liquids by NMR Signal Amplification by Reversible Exchange, J Phys Chem Lett, 6 (2015) 1961-1967.

[62] R.V. Shchepin, D.A. Barskiy, D.M. Mikhaylov, E.Y. Chekmenev, Efficient Synthesis of Nicotinamide-1-N for Ultrafast NMR Hyperpolarization Using Parahydrogen, Bioconjugate chemistry, (2016).

[63] A.N. Pravdivtsev, A.V. Yurkovskaya, H. Zimmermann, H.M. Vieth, K.L. Ivanov, Transfer of SABREderived hyperpolarization to spin-1/2 heteronuclei, Rsc Adv, 5 (2015) 63615-63623.

[64] A.N. Pravdivtsev, A.V. Yurkovskaya, N.N. Lukzen, K.L. Ivanov, H.M. Vieth, Highly Efficient Polarization of Spin-1/2 Insensitive NMR Nuclei by Adiabatic Passage through Level Anticrossings, J Phys Chem Lett, 5 (2014) 3421-3426.

[65] S.R. Hartmann, E.L. Hahn, Nuclear Double Resonance in the Rotating Frame, Physical Review, 128 (1962) 2042-2053.

[66] A.N. Pravdivtsev, A.V. Yurkovskaya, H.M. Vieth, K.L. Ivanov, Spin mixing at level anti-crossings in the rotating frame makes high-field SABRE feasible, Phys Chem Chem Phys, 16 (2014) 24672-24675.

[67] A.N. Pravdivtsev, A.V. Yurkovskaya, H.M. Vieth, K.L. Ivanov, RF-SABRE: A Way to Continuous Spin Hyperpolarization at High Magnetic Fields, J Phys Chem B, 119 (2015) 13619-13629.

[68] T. Theis, M. Truong, A.M. Coffey, E.Y. Chekmenev, W.S. Warren, LIGHT-SABRE enables efficient in-magnet catalytic hyperpolarization, J Magn Reson, 248 (2014) 23-26.

[69] Q. Gong, A. Gordji-Nejad, B. Blümich, S. Appelt, Trace analysis by low-field NMR: breaking the sensitivity limit, Anal Chem, 82 (2010) 7078-7082.

[70] S. Glöggler, R. Müller, J. Colell, M. Emondts, M. Dabrowski, B. Blümich, S. Appelt, Para-hydrogen induced polarization of amino acids, peptides and deuterium-hydrogen gas, Phys Chem Chem Phys, 13 (2011) 13759-13764. 
[71] T. Trantzschel, M. Plaumann, J. Bernarding, D. Lego, T. Ratajczyk, S. Dillenberger, G. Buntkowsky, J. Bargon, U. Bommerich, Application of Parahydrogen-Induced Polarization to Unprotected Dehydroamino Carboxylic Acids, Appl Magn Reson, 44 (2013) 267-278.

[72] R. Borowiak, N. Schwaderlapp, F. Huethe, T. Lickert, E. Fischer, S. Bar, J. Hennig, D. von Elverfeldt, J.B. Hovener, A battery-driven, low-field NMR unit for thermally and hyperpolarized samples, Magn Reson Mater Phy, 26 (2013) 491-499.

[73] J.B. Hövener, N. Schwaderlapp, T. Lickert, S.B. Duckett, R.E. Mewis, L.A. Highton, S.M. Kenny, G.G. Green, D. Leibfritz, J.G. Korvink, J. Hennig, D. von Elverfeldt, A hyperpolarized equilibrium for magnetic resonance, Nat Commun, 4 (2013) 2946.

[74] D.A. Barskiy, K.V. Kovtunov, I.V. Koptyug, P. He, K.A. Groome, Q.A. Best, F. Shi, B.M. Goodson, R.V. Shchepin, M.L. Truong, A.M. Coffey, K.W. Waddell, E.Y. Chekmenev, In Situ and Ex Situ Low-Field NMR Spectroscopy and MRI Endowed by SABRE Hyperpolarization, Chemphyschem, 15 (2014) 41004107.

[75] T. Theis, M.P. Ledbetter, G. Kervern, J.W. Blanchard, P.J. Ganssle, M.C. Butler, H.D. Shin, D. Budker, A. Pines, Zero-field NMR enhanced by parahydrogen in reversible exchange, J Am Chem Soc, 134 (2012) 3987-3990.

[76] F. Shi, A.M. Coffey, K.W. Waddell, E.Y. Chekmenev, B.M. Goodson, Heterogeneous Solution NMR Signal Amplification by Reversible Exchange, Angew Chem Int Edit, 53 (2014) 7495-7498.

[77] F. Shi, A.M. Coffey, K.W. Waddell, E.Y. Chekmenev, B.M. Goodson, Nanoscale Catalysts for NMR Signal Enhancement by Reversible Exchange, J Phys Chem C, 119 (2015) 7525-7533.

[78] K.L. Ivanov, A.N. Pravdivtsev, A.V. Yurkovskaya, H.M. Vieth, R. Kaptein, The role of level anticrossings in nuclear spin hyperpolarization, Prog Nucl Mag Res Sp, 81 (2014) 1-36.

[79] A.N. Pravdivtsev, A.V. Yurkovskaya, H.M. Vieth, K.L. Ivanov, Coherent transfer of nuclear spin polarization in field-cycling NMR experiments, J Chem Phys, 139 (2013).

[80] A.N. Pravdivtsev, K.L. Ivanov, A.V. Yurkovskaya, P.A. Petrov, H.H. Limbach, R. Kaptein, H.M. Vieth, Spin polarization transfer mechanisms of SABRE: A magnetic field dependent study, J Magn Reson, 261 (2015) 73-82.

[81] D.A. Barskiy, A.N. Pravdivtsev, K.L. Ivanov, K.V. Kovtunov, I.V. Koptyug, A simple analytical model for signal amplification by reversible exchange (SABRE) process, Phys Chem Chem Phys, 18 (2016) 8993.

[82] S. Knecht, A.N. Pravdivtsev, J.-B. Hovener, A.V. Yurkovskaya, K.L. Ivanov, Quantitative description of the SABRE process: rigorous consideration of spin dynamics and chemical exchange, Rsc Adv, 6 (2016) 24470-24477.

[83] N. Eshuis, R.L. Aspers, B.J. van Weerdenburg, M.C. Feiters, F.P. Rutjes, S.S. Wijmenga, M. Tessari, Determination of long-range scalar $(1) \mathrm{H}-(1) \mathrm{H}$ coupling constants responsible for polarization transfer in SABRE, J Magn Reson, 265 (2016) 59-66.

[84] J.B. Hövener, S. Knecht, N. Schwaderlapp, J. Hennig, D. von Elverfeldt, Continuous rehyperpolarization of nuclear spins using parahydrogen: theory and experiment, Chemphyschem, 15 (2014) 2451-2457.

[85] B.J. van Weerdenburg, A.H. Engwerda, N. Eshuis, A. Longo, D. Banerjee, M. Tessari, C.F. Guerra, F.P. Rutjes, F.M. Bickelhaupt, M.C. Feiters, Computational (DFT) and Experimental (EXAFS) Study of the Interaction of $[\operatorname{Ir}(\mathrm{IMes})(\mathrm{H}) 2(\mathrm{~L}) 3$ ] with Substrates and Co-substrates Relevant for SABRE in Dilute Systems, Chemistry, 21 (2015) 10482-10489.

[86] J.A. Osborn, R.R. Schrock, Preparation and properties of some cationic complexes of rhodium(I) and rhodium(III), J Am Chem Soc, 93 (1971) 2397-2407.

[87] J.A. Osborn, R.R. Schrock, Coordinatively unsaturated cationic complexes of rhodium(I), iridium(I), palladium(II), and platinum(II). Generation, synthetic utility, and some catalytic studies, J Am Chem Soc, 93 (1971) 3089-3091. 
[88] Y. Xu, D.M.P. Mingos, J.M. Brown, Crabtree's catalyst revisited; Ligand effects on stability and durability, Chem Commun, (2008) 199-201.

[89] J.J. Verendel, O. Pàmies, M. Diéguez, P.G. Andersson, Asymmetric Hydrogenation of Olefins Using Chiral Crabtree-type Catalysts: Scope and Limitations, Chemical Reviews, 114 (2014) 21302169.

[90] M.N. Hopkinson, C. Richter, M. Schedler, F. Glorius, An overview of N-heterocyclic carbenes, Nature, 510 (2014) 485-496.

[91] M. Melaimi, M. Soleilhavoup, G. Bertrand, Stable Cyclic Carbenes and Related Species beyond Diaminocarbenes, Angewandte Chemie International Edition, 49 (2010) 8810-8849.

[92] S. Bellemin-Laponnaz, S. Dagorne, Group 1 and 2 and Early Transition Metal Complexes Bearing $\mathrm{N}$-Heterocyclic Carbene Ligands: Coordination Chemistry, Reactivity, and Applications, Chemical Reviews, 114 (2014) 8747-8774.

[93] W.A. Herrmann, N-Heterocyclic Carbenes: A New Concept in Organometallic Catalysis, Angewandte Chemie International Edition, 41 (2002) 1290-1309.

[94] S. Díez-González, N. Marion, S.P. Nolan, N-Heterocyclic Carbenes in Late Transition Metal Catalysis, Chemical Reviews, 109 (2009) 3612-3676.

[95] A.J. Arduengo, R.L. Harlow, M. Kline, A stable crystalline carbene, J Am Chem Soc, 113 (1991) 361-363.

[96] L. Delaude, Betaine Adducts of N-Heterocyclic Carbenes: Synthesis, Properties, and Reactivity, European Journal of Inorganic Chemistry, 2009 (2009) 1681-1699.

[97] M.J. Fuchter, N-Heterocyclic Carbene Mediated Activation of Tetravalent Silicon Compounds: A Critical Evaluation, Chemistry - A European Journal, 16 (2010) 12286-12294.

[98] D.P. Curran, A. Solovyev, M. Makhlouf Brahmi, L. Fensterbank, M. Malacria, E. Lacôte, Synthesis and Reactions of $\mathrm{N}$-Heterocyclic Carbene Boranes, Angewandte Chemie International Edition, 50 (2011) 10294-10317.

[99] E.L. Kolychev, E. Theuergarten, M. Tamm, N-Heterocyclic Carbenes in FLP Chemistry, in: G. Erker, W.D. Stephan (Eds.) Frustrated Lewis Pairs II: Expanding the Scope, Springer Berlin Heidelberg, Berlin, Heidelberg, 2013, pp. 121-155.

[100] Y. Wang, Y. Xie, P. Wei, R.B. King, H.F. Schaefer, P. von R. Schleyer, G.H. Robinson, A Stable Silicon(0) Compound with a Si=Si Double Bond, Science, 321 (2008) 1069-1071.

[101] Y. Wang, Y. Xie, P. Wei, R.B. King, I.I.I.H.F. Schaefer, P.v.R. Schleyer, G.H. Robinson, CarbeneStabilized Diphosphorus, J Am Chem Soc, 130 (2008) 14970-14971.

[102] D. Martin, M. Soleilhavoup, G. Bertrand, Stable singlet carbenes as mimics for transition metal centers, Chem Sci, 2 (2011) 389-399.

[103] C.D. Martin, M. Soleilhavoup, G. Bertrand, Carbene-stabilized main group radicals and radical ions, Chem Sci, 4 (2013) 3020-3030.

[104] X. Bugaut, F. Glorius, Organocatalytic umpolung: N-heterocyclic carbenes and beyond, Chem Soc Rev, 41 (2012) 3511-3522.

[105] X.-Y. Chen, S. Ye, N-heterocyclic carbene-catalyzed reactions of C-C unsaturated bonds, Org Biomol Chem, 11 (2013) 7991-7998.

[106] M. Fevre, J. Pinaud, Y. Gnanou, J. Vignolle, D. Taton, N-Heterocyclic carbenes (NHCs) as organocatalysts and structural components in metal-free polymer synthesis, Chem Soc Rev, 42 (2013) 2142-2172.

[107] F.E. Hahn, M.C. Jahnke, Heterocyclic Carbenes: Synthesis and Coordination Chemistry, Angewandte Chemie International Edition, 47 (2008) 3122-3172.

[108] N. Kuhn, A. Al-Sheikh, 2,3-Dihydroimidazol-2-ylidenes and their main group element chemistry, Coordin Chem Rev, 249 (2005) 829-857. 
[109] P.L. Arnold, I.J. Casely, F-Block N-Heterocyclic Carbene Complexes, Chemical Reviews, 109 (2009) 3599-3611.

[110] A.C. Hillier, W.J. Sommer, B.S. Yong, J.L. Petersen, L. Cavallo, S.P. Nolan, A Combined Experimental and Theoretical Study Examining the Binding of $\mathrm{N}$-Heterocyclic Carbenes (NHC) to the $\mathrm{Cp} * \mathrm{RuCl}\left(\mathrm{Cp}^{*}=\eta 5-\mathrm{C} 5 \mathrm{Me} 5\right)$ Moiety: Insight into Stereoelectronic Differences between Unsaturated and Saturated NHC Ligands, Organometallics, 22 (2003) 4322-4326.

[111] C.M. Crudden, D.P. Allen, Stability and reactivity of N-heterocyclic carbene complexes, Coordin Chem Rev, 248 (2004) 2247-2273.

[112] A.V. Zhukhovitskiy, M.G. Mavros, T. Van Voorhis, J.A. Johnson, Addressable Carbene Anchors for Gold Surfaces, J Am Chem Soc, 135 (2013) 7418-7421.

[113] P. Lara, O. Rivada-Wheelaghan, S. Conejero, R. Poteau, K. Philippot, B. Chaudret, Ruthenium Nanoparticles Stabilized by N-Heterocyclic Carbenes: Ligand Location and Influence on Reactivity, Angewandte Chemie International Edition, 50 (2011) 12080-12084.

[114] A.T. Normand, K.J. Cavell, Donor-Functionalised N-Heterocyclic Carbene Complexes of Group 9 and 10 Metals in Catalysis: Trends and Directions, European Journal of Inorganic Chemistry, 2008 (2008) 2781-2800.

[115] N. Marion, S.P. Nolan, N-Heterocyclic carbenes in gold catalysis, Chem Soc Rev, 37 (2008) 17761782.

[116] E.A.B. Kantchev, C.J. O'Brien, M.G. Organ, Palladium Complexes of N-Heterocyclic Carbenes as Catalysts for Cross-Coupling Reactions-A Synthetic Chemist's Perspective, Angewandte Chemie International Edition, 46 (2007) 2768-2813.

[117] G.C. Fortman, S.P. Nolan, N-Heterocyclic carbene (NHC) ligands and palladium in homogeneous cross-coupling catalysis: a perfect union, Chem Soc Rev, 40 (2011) 5151-5169.

[118] S. Würtz, F. Glorius, Surveying Sterically Demanding N-Heterocyclic Carbene Ligands with Restricted Flexibility for Palladium-catalyzed Cross-Coupling Reactions, Accounts Chem Res, 41 (2008) 1523-1533.

[119] G.C. Vougioukalakis, R.H. Grubbs, Ruthenium-Based Heterocyclic Carbene-Coordinated Olefin Metathesis Catalysts, Chemical Reviews, 110 (2010) 1746-1787.

[120] C. Samojłowicz, M. Bieniek, K. Grela, Ruthenium-Based Olefin Metathesis Catalysts Bearing NHeterocyclic Carbene Ligands, Chemical Reviews, 109 (2009) 3708-3742.

[121] L. Novotny, Strong coupling, energy splitting, and level crossings: A classical perspective, Am J Phys, 78 (2010) 1199-1202.

[122] E.B. Dücker, Non-Hydrogenative Parahydrogen-Induced Polarization on Pyrazole Derivatives, in, Georg-August-Universität Göttingen, Göttingen, 2010.

[123] A.R. Cochrane, S. Irvine, W.J. Kerr, M. Reid, S. Andersson, G.N. Nilsson, Application of neutral iridium(I) N-heterocyclic carbene complexes in ortho-directed hydrogen isotope exchange, J Labelled Compd Rad, 56 (2013) 451-454.

[124] D. Hesk, P.R. Das, B. Evans, Deuteration of Acetanilides and Other Substituted Aromatics Using [Ir(Cod)(Cy(3)P)(Py)]Pf6 as Catalyst, J Labelled Compd Rad, 36 (1995) 497-502.

[125] A. Albert, J.N. Phillips, 264. Ionization constants of heterocyclic substances. Part II. Hydroxyderivatives of nitrogenous six-membered ring-compounds, Journal of the Chemical Society (Resumed), (1956) 1294-1304.

[126] A. Güven, Acidity study on 3-substituted pyridines, Int J Mol Sci, 6 (2005) 257-275.

[127] J. Llor, O. Lopez-Mayorga, L. Muñoz, Thermodynamic characterization of tautomeric equilibria by multinuclear magnetic resonance. Application to 3-hydroxypyridine, Magnetic Resonance in Chemistry, 31 (1993) 552-556. 
[128] D. Berry, K. Bukka, R.S. Satchell, Quantitative aspects of Lewis acidity. Part XV. Basicity of substituted pyridines and quinolines towards metal halides in diethyl ether, Journal of the Chemical Society, Perkin Transactions 2, (1976) 89-91.

[129] E. Spinner, 716. The electronic spectra of some monosubstituted pyridines and pyridinium ions, Journal of the Chemical Society (Resumed), (1963) 3855-3859.

[130] S. Belanger, M.H. Keefe, J.L. Welch, J.T. Hupp, Rapid derivatization of mesoporous thin-film materials based on $\mathrm{Re}(\mathrm{I})$ zinc-porphyrin 'molecular squares': selective modification of mesopore size and shape by binding of aromatic nitrogen donor ligands, Coordin Chem Rev, 192 (1999) 29-45.

[131] I. lavicoli, V. Cufino, M. Corbi, M. Goracci, E. Caredda, A. Cittadini, A. Bergamaschi, A. Sgambato, Rhodium and iridium salts inhibit proliferation and induce DNA damage in rat fibroblasts in vitro, Toxicology in vitro : an international journal published in association with BIBRA, 26 (2012) 963-969.

[132] D.P. Allen, M.M. Van Wingerden, R.H. Grubbs, Well-Defined Silica-Supported Olefin Metathesis Catalysts, Org Lett, 11 (2009) 1261-1264.

[133] S. Warsink, J.A. Venter, A. Roodt, NHC-amide donor ligands in rhodium complexes: Syntheses and characterisation, Journal of Organometallic Chemistry, 775 (2015) 195-201.

[134] A. Reisinger, N. Trapp, I. Krossing, AgOC(CF3)3: Synthesis and Applications of the First DonorFree Silver(I) Alkoxide, Organometallics, 26 (2007) 2096-2105. 


\section{List of Figures}

Figure 1: Setup of the Bruker Parahydrogen Polariser. Using the syringe, the sample solution can be transferred into the polarization chamber, where the polarization field can be adjusted by the surrounding solenoid. To achieve dissolution of $p \mathrm{H}_{2}$, a flow of the gas can be applied to the mixing chamber and subsequently the solution can be transferred into an attached flow probe for NMR examination.

Figure 2: Assorted ligands utilized in SABRE related studies including IMes (1), SIMes (2), PCyз (5) and $\mathrm{PPh}_{3}(6)$.

Figure 3: Assorted modified phosphine and NHC ligands. Ligands $\mathbf{7 a - c ,} \mathbf{8}$ and $\mathbf{9 a , b}$ were synthesized to create polarization transfer catalysts with a higher polarity to be used in water-based solvent systems, while ligand $\mathbf{1 0}$ served the purpose of creating a neutral catalytic complex for application of SABRE in non-polar solvents.

Figure 4: Molecular structures of chemical compounds referred to in this section.

Figure 5: Chemical structures of pyridine derivatives, into which phosphorus functionalities were introduced to be examined in ref. [59].

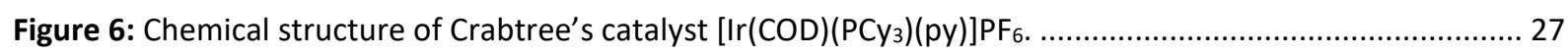

Figure 7: Chemical structure of 1,3-di(adamantyl)imidazole-2-ylidene. ........................................................ 27

Figure 8: Different classes of familiar NHCs exhibiting structural and electronic differences............................. 28

Figure 9: Thermal equilibrium parahydrogen fraction in $\mathrm{H}_{2}$ gas in dependence of the temperature.................. 30

Figure 10: $1 \mathrm{H}$ NMR spectrum of an activated polarization transfer catalyst $\mathbf{C}$ (Scheme 4). Resonances between 1 and 2 ppm arise from the employed phosphine ligand tricyclohexyl phosphine while the peaks in the aromatic region originate from the substrate pyridine, either in its free form or as a ligand in the iridium complex. The resonance at $-23.5 \mathrm{ppm}$ stems from the hydride ligands. Remaining signals arise from water, hydrogen gas or the solvent MeOD.

Figure 11: Oscillation frequencies of systems $A$ and $B$ depending on the spring constant of system $B$ with spring constants $k A=k 0$ and $k B=k 0+\Delta k$. In (a), no coupling is present between the oscillators and the oscillation frequencies are independent of each other. In (b), a coupling of $\kappa=0.08 k 0$ was introduced between the oscillators. Under these conditions, a crossing of the oscillation frequencies is avoided and their trajectories exhibit a characteristic frequency splitting of $\Gamma=\kappa / m A \kappa / m B \omega A \omega B$. This figure was adapted from [121].....

Figure 12: Chemical structure and NMR data of trans-2-butene.

Figure 13: Three-spin model complex for the discussion of the SABRE polarization transfer mechanism based on the equatorial arrangement of the active polarization transfer catalyst. $A$ and $A^{\prime}$ denote the hydride spins originating from a parahydrogen molecule whereas $M$ represents a spin in the substrate molecule. The iridium nucleus is NMR inactive and serves as a connecting piece.

Figure 14: Four-spin model complex consisting of the hydride spin pair $\mathrm{AA}^{\prime}$ and the substrate spin pair $M M^{\prime}$. In this model, $J_{A, A^{\prime}}$ is considerably larger than the remaining scalar couplings and the individual hydride spins exhibit inequivalent couplings to the two substrate spins $\left(J_{A, M} \neq J_{A, M^{\prime}}\right)$. Furthermore, $J_{A, M}=J_{A^{\prime}, M^{\prime}}$ and $J_{A^{\prime}, M}=J_{A, M^{\prime}}$. The iridium nucleus is NMR inactive and therefore disregarded.

Figure 15: Calculated field dependences of SABRE attained for the three- and four-spin model systems $A A^{\prime} M$ and $A A^{\prime} M M^{\prime}$ using the NMR parameters given on the right-hand side. (A) depicts the results for the $A A^{\prime} M$ three-spin system, for which only a single LAC was determined, and which gives rise to a single emissive maximum. The results for the $A A^{\prime} M M^{\prime}$ four-spin system are given in (B). Here, two locations of LACs were identified, which, however are not 
separated and form a single emissive maximum. This figure was reproduced from the supplementary data of ref. [37].

Figure 16: Structure of the five-spin model complex $A A^{\prime} M M^{\prime} P$ and calculated field dependence of SABRE obtained using the NMR parameters on the right-hand side. As a result of coupling of the ${ }^{31} \mathrm{P}$ nucleus in the phosphine ligand to the hydride spins, the number of LACs is doubled compared to the $A A^{\prime} M M^{\prime}$ four-spin model. Due to their relative proximity, pairs of two LACs overlap and give a combined maximum, resulting in only one absorptive and one emissive maximum from four level anti-crossings. This figure was reproduced from ref. [37].

Figure 17: Polarization of the substrate spin $\mathrm{M}$ as a result of the interconversion between $S \alpha$ and $T+\beta$ as a function of the complex lifetime. For a larger scalar coupling difference $J A, B-J A^{\prime}, B$, the rate of oscillation is increased.

Figure 18: Polarization field dependence of signal enhancement for the protons in pyrazole without pyridine- $d_{5}$ as a secondary substrate. The molecular structure of the substrate molecule is given on the right.

Figure 19: Polarization field dependence of signal enhancement for the protons in pyrazole with pyridine- $d_{5}$ as a secondary substrate. The molecular structure of the substrate molecule is given on the right.

Figure 20: Polarization field dependence of signal enhancement for the protons in 3,5-dimethylpyrazole. The molecular structure of the substrate molecule is given on the right.

Figure 21: Polarization field dependence of signal enhancement for the protons in 4-methylpyrazole in the free state and when coordinated to the polarization transfer catalyst. The molecular structure of the substrate molecule is given on the right.

Figure 22: Polarization field dependence of signal enhancement for the protons in positions 4 and 5 of 3-aminopyrazole. The molecular structure of the substrate molecule is given on the right....

Figure 23: Polarization field dependence for the pyrazole protons in 3-(4-aminophenyl)pyrazole. The molecular structure of the substrate molecule is given on the right.

Figure 24: Polarization field dependence of signal enhancement for the protons in 3,4-dimethylpyrazole. The molecular structure of the substrate molecule is given at the top right.

Figure 25: Polarization field dependence of signal enhancement for the protons in 4-ethoxy-3methylpyrazole. The molecular structure of the substrate molecule is given on the right.

Figure 26: Polarization field dependence of signal enhancement for the protons in indazole. The molecular structure of the substrate molecule is given at the top right.

Figure 27: Polarization field dependence of signal enhancement for the protons in 1,2,3-triazole and 1,2,4-triazole. The molecular structures of the substrate molecules are given on the right.

Figure 28: Binding motifs of 1,2,4-triazole to the iridium center in the active polarization transfer complex. Further ligands in the iridium complex are omitted.

Figure 29: Polarization field dependence of signal enhancement for the protons in nicotinamide. The molecular structure of the substrate molecule is given on the right

Figure 30: Polarization field dependence of signal enhancement for protons in 3,4,5-trideuteropyridine. The molecular structure of the substrate molecule is given on the right.

Figure 31: Polarization field dependence of signal enhancement for the meta and para protons of 2,6-dideuteropyridine. The molecular structure of the substrate molecule is given on the right.

Figure 32: Polarization field dependence of signal enhancement for the protons in pyridazine. The molecular structure of the substrate molecule is given on the right 


\section{List of Figures}

Figure 33: Exemplary spectrum overlay of the aromatic region of nicotinamide. For the hyperpolarized spectrum, the polarization step was performed at a field of $40 \mathrm{Gs}$. The reference spectrum is enlarged by a factor of 16 . Unlabeled resonances either originate from pyridine $(8.55 \mathrm{ppm})$ or from complex-coordinated substrates (8.95 ppm).

Figure 34: Deviations from the magnetic field determined at the center of the sample. The asymmetry is owed to the presence of the gas valve at the top end of the sample tube.

Figure 35: Polarization field dependence of signal enhancement for the protons in 3-methylpyrazole. The molecular structure of the substrate molecule is given at the top right.

Figure 36: Polarization field dependence of signal enhancement for the protons pyridine. The molecular structure of the substrate molecule is given at the top right.

Figure 37: Polarization field dependence of signal enhancement for the protons in 3-phenylpyrazole. The molecular structure of the substrate molecule is given at the top right.

Figure 38: Polarization field dependence of signal enhancement for the protons in 1,2,4-triazole. The molecular structure of the substrate molecule is given on the right

Figure 39: Polarization field dependence of signal enhancement for the protons in indazole. The molecular structure of the substrate molecule is given at the top right.

Figure 40: Polarization field dependence of signal enhancement for the protons in 4-ethoxy-3methylpyrazole. The molecular structure of the substrate molecule is given on the right.

Figure 41: Polarization field dependence of signal enhancement for the protons in 2,6dideuteropyridine. The molecular structure of the substrate molecule is given on the right.

Figure 42: Polarization field dependence of signal enhancement for the protons in nicotinamide. The molecular structure of the substrate molecule is given on the right.

Figure 43: Polarization field dependence of signal enhancement for the protons in quinoline. The molecular structure of the substrate molecule is given at the top left.

Figure 44: Polarization field dependence of signal enhancement for the protons in 4-methylpyrazole determined with various catalysts. The molecular structure of the substrate molecule is given on the right.

Figure 45: Polarization field dependence of signal enhancement for the protons in 3,5-dimethylpyrazole determined with two different catalysts. The molecular structure of the substrate molecule is given on the right.

Figure 46: Polarization field dependence of signal enhancement for the protons in 3-methylpyridine determined with various catalysts. The molecular structure of the substrate molecule is given at the top right.

Figure 47: Polarization field dependence of signal enhancement for the protons in 3-methoxypyridine determined with various catalysts. The molecular structure of the substrate molecule is given at the top right.

Figure 48: Polarization field dependence of signal enhancement for the protons in 3-hydroxypyridine determined with various catalysts. The molecular structure of the substrate molecule is given on the right.

Figure 49: Polarization field dependence of signal enhancement for the protons in 3-fluoropyridine determined with various catalysts. The molecular structure of the substrate molecule is given on the right.

Figure 50: Polarization field dependence of signal enhancement for the protons in 3-chloropyridine determined with various catalysts. The molecular structure of the substrate molecule is given on the right. 
Figure 51: Maximum enhancements of the protons in 3-chloropyridine achieved using the $[\operatorname{Ir}(\mathrm{IMes})(\mathrm{COD}) \mathrm{Cl}]$ precatalyst in dependence of the level of dilution.

Figure 52: Polarization field dependence of signal enhancement for the protons in 3-bromopyridine determined with various catalysts. The molecular structure of the substrate molecule is given on the right.

Figure 53: Polarization field dependence of signal enhancement for the protons in 3-iodopyridine determined with various catalysts. The molecular structure of the substrate molecule is given on the right.

Figure 54: Polarization field dependence of signal enhancement for the protons in 3,5-dichloropyridine determined with various catalysts. The molecular structure of the substrate molecule is given on the right.

Figure 55: Polarization field dependence of signal enhancement for the protons in 4-iodopyridine determined with various catalysts. The molecular structure of the substrate molecule is given on the right.

Figure 56: Polarization field dependence of signal enhancement for the protons in 3,5-dibromopyridine determined with various catalysts. The molecular structure of the substrate molecule is given on the right.

Figure 57: Polarization field dependence of signal enhancement for the protons in 3,5-dimethylpyridine determined with various catalysts. The molecular structure of the substrate molecule is given at the top right.

Figure 58: Polarization field dependence of signal enhancement for the protons in 3,4-dimethylpyridine determined with various catalysts. The molecular structure of the substrate molecule is given at the top right.

Figure 59: Exemplary spectrum overlay of the aromatic region of 3-chloropyridine. The hyperpolarized spectrum was recorded at a polarizer setting of $-50 \mathrm{Gs}$, resulting in an effective polarization field of $52 \mathrm{Gs}$. The reference spectrum is enhanced by a factor of 128 . Additional resonances in the hyperpolarized spectra originate from hyperpolarized substrate molecules coordinated to the polarization transfer catalyst.

Figure 60: Signal intensities of the protons in 3-chloropyridine in dependence of the number of executed sample transfers. Due to the limited time in the spectrometer, the Boltzmann polarization is not reached, resulting in relative signal intensities $<1$. Differences in the signal intensities between individual protons stem from the individual relaxation times.

Figure 61: Level of enhancement achieved for the protons in 3-chloropyridine at a field of 5 Gs in dependence of the number of experiments performed. The IMes polarization transfer catalyst was used to facilitate the hyperpolarization.

Figure 62: Polarization field dependence for $\mathrm{H} 2$ in 3-chloropyridine using the IMes polarization transfer catalyst. Both field dependence profiles were recorded in direct succession using identical sample compositions and parameters with the exception of the direction in which the polarization field was modulated.

Figure 63: Signal intensity for the protons in 3-chloropyridine in dependence of the number of performed sample transfers. The observed transfer of hyperpolarization is achieved by residual catalysts within the tubing system that withstood an extended washing operation.

Figure 64: Deviations from the selected polarization field over the effective sample volume in the mixing chamber. Percentage deviations are referenced to the appointed field at the lowest of three characteristic points of the sample volume. Absolute field deviations are given as the difference between highest and lowest field at these three locations. 


\section{List of Figures}

Figure 65: Structure of $[\mathrm{IrCl}(\mathrm{COD})(\mathrm{IMes})]$ containing the IMes ligand. The complex reacts with suitable substrates and $\mathrm{pH}_{2}$ to generate an active polarization transfer catalyst.

Figure 66: Compound 1 was appointed as the initial target molecule for the synthesis of a solid-phase bound ligand to be used in SABRE hyperpolarization experiments.

Figure 67: Compounds $\mathbf{9}$ and $\mathbf{1 0}$ were appointed as new synthesis targets.

Figure 68: Compounds $\mathbf{1 1}$ and $\mathbf{1 2}$ are the respective isolated precursors of target molecules $\mathbf{9}$ and $\mathbf{1 0 .}$ 83

Figure 69: ${ }^{1} \mathrm{H}$ NMR spectra section of solid-phase bound benzyl bromide 14 (red) and imidazolinium bromide 16 (blue). Formation of 16 is clearly indicated by emergence of the indicated methyl resonances between 2.5 and $2.0 \mathrm{ppm}$ and the methine resonance at $4.8 \mathrm{ppm}$, while the intensity of the methine resonance at $4.5 \mathrm{ppm}$ is significantly reduced.

Figure 70: Hydrogenation of the double bond in 4-allyl-2,6-dimethylaniline to a single bond to give 2,6dimethyl-4-propylaniline.

Figure 71: Spectra recorded in SABRE experiments using solid-phase bound catalyst 20 and pyridine as the analyte. The red spectrum displays the thermal equilibrium reference, while the blue spectrum was recorded after exposure of the sample solution and the insoluble catalyst particles to a flow of parahydrogen gas for 30 seconds.

Figure 72: Movable platform holding a sample mounting and the electromotor used to propel the reciprocating motion.

Figure 73: Position of the table construction at the $600 \mathrm{MHz}$ spectrometer. The platform on top of the table was movable along the length of the top beam, allowing for the positioning of the sample at various field strengths within the stray field of the NMR magnet.

Figure 74: Strength of the magnetic stray field generated by the Bruker Avance III $600 \mathrm{MHz}$ magnet in dependence of the distance from the spectrometer hull.

Figure 75: Simplified sketch of experimental setup 2 using the Bruker Para-Hydrogen Polariser and a Bruker Avance III $600 \mathrm{MHz}$ spectrometer.

Figure 76: The mixing chamber of the Para-Hydrogen Polariser with all attached tubing connections. The height marks denote the distance from the upper rim auf the electromagnetic coil inside which the mixing chamber is placed. Bz denotes the $z$-component of the magnetic field generated by the NMR magnet as well as by the solenoid coil surrounding the mixing chamber.

Figure 77: Sketch of the dummy flow cell used to determine the transfer parameters. The arrow markings indicate the optimal liquid level at the end of the respective transfer phase.

Figure 78: Effective polarization field at different positions inside the mixing chamber in dependence of the magnetic field induced by the electromagnetic coil.

Figure 79: Molecular structure and numbering of the substrate molecule pyrazole......................................99

Figure 80: Molecular structure and numbering of the substrate molecule 3-methylpyrazole. ...........................98

Figure 81: Molecular structure and numbering of the substrate molecule 4-methylpyrazole. ........................98

Figure 82: Molecular structure and numbering of the substrate molecule 3,4-dimethylpyrazole. ....................98

Figure 83: Molecular structure and numbering of the substrate molecule 3,5-dimethylpyrazole. ....................98

Figure 84: Molecular structure and numbering of the substrate molecule 3-phenylpyrazole.......................... 98

Figure 85: Molecular structure and numbering of the substrate molecule 3-(4-aminophenyl)pyrazole............. 99

Figure 86: Molecular structure and numbering of the substrate molecule 3,5-diphenylpyrazole..................... 99

Figure 87: Molecular structure and numbering of the substrate molecule 3,5-ditertbutylpyrazole...................99

Figure 88: Molecular structure and numbering of the substrate molecule 3-aminopyrazole. ......................... 99

Figure 89: Molecular structure and numbering of the substrate molecule 4-ethoxy-3-methylpyrazole............ 99 
Figure 90: Molecular structure and numbering of the substrate molecule indazole. ................................... 100

Figure 91: Molecular structure and numbering of the substrate molecule 1,2,3-triazole. .............................. 100

Figure 92: Molecular structure and numbering of the substrate molecule 1,2,4-triazole. ............................. 100

Figure 93: Molecular structure and numbering of the substrate molecule pyridine. ....................................... 100

Figure 94: Molecular structure and numbering of the substrate molecule 2,6-dideuteropyridine. ................. 100

Figure 95: Molecular structure and numbering of the substrate molecule 3,4,5-trideuteropyridine............... 101

Figure 96: Molecular structure and numbering of the substrate molecule nicotinamide. .............................. 101

Figure 97: Molecular structure and numbering of the substrate molecule 3-fluoropyridine. ......................... 101

Figure 98: Molecular structure and numbering of the substrate molecule 3-chloropyridine.......................... 101

Figure 99: Molecular structure and numbering of the substrate molecule 3-bromopyridine. ......................... 102

Figure 100: Molecular structure and numbering of the substrate molecule 3-iodopyridine........................... 102

Figure 101: Molecular structure and numbering of the substrate molecule 3-methylpyridine......................... 102

Figure 102: Molecular structure and numbering of the substrate molecule 3-methoxypyridine.................... 102

Figure 103: Molecular structure and numbering of the substrate molecule 3-hydroxypyridine. ..................... 102

Figure 104: Molecular structure and numbering of the substrate molecule 3,5-dichloropyridine................... 103

Figure 105: Molecular structure and numbering of the substrate molecule 3,5-dibromopyridine. ................. 103

Figure 106: Molecular structure and numbering of the substrate molecule 3,5-dimethylpyridine.................. 103

Figure 107: Molecular structure and numbering of the substrate molecule 3,4-dimethylpyridine................. 103

Figure 108: Molecular structure and numbering of the substrate molecule pyridazine.................................. 104

Figure 109: Molecular structure and numbering of the substrate molecule quinoline. ................................. 104

Figure 110: Molecular structure and numbering of the substrate molecule 4-bromo-2,8-

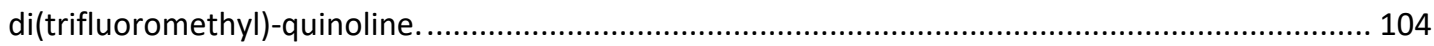

Figure 111: Molecular structure and numbering of the substrate molecule dibenzothiophene. ...................... 104 


\section{List of Schemes}

\section{List of Schemes}

Scheme 1: Differences between PHIP and SABRE. In reactions A and B, the parahydrogen nuclei are transferred into coupled, yet unequal positions in the product compounds indicated by the distinguishable substituents $R$ and $R^{\prime}$ as well as the ligands $L$ and $L^{\prime}$. Consequentially, the polarization on the nuclei becomes observable and enhanced resonances of the highlighted proton spins can be recorded by NMR spectroscopy. In reaction $\mathrm{C}$, the substrate and the $\mathrm{pH}_{2}$ nuclei are only temporarily associated via a metal template, resulting in a transfer of polarization to the spins of the unmodified substrate, observable by NMR. [M] indicates a non-specified metal coordination complex.

Scheme 2: Preparation of the active polarization transfer catalyst starting from the precatalysts $[\operatorname{Ir}(\mathrm{COD})(\mathrm{PCy})(\mathrm{py})]^{+}$and $[\operatorname{Ir}(\mathrm{COD})(\mathrm{NHC}) \mathrm{Cl}](\mathrm{NHC}=\mathrm{IMes}$, SIMes). Here, pyridine is used as an exemplary substrate compound.

Scheme 3: Simplified schematic of the catalytic cycle of the SABRE polarization transfer. The highlighted molecules are in a non-equilibrium nuclear spin state and are therefore considered hyperpolarized. Pyridine is depicted as an exemplary substrate.

Scheme 4: Preparation of the active polarization transfer catalyst starting from the precatalysts $[\operatorname{Ir}(\mathrm{COD})(\mathrm{NHC}) \mathrm{Cl}](\mathrm{NHC}=\mathrm{IMes}, \mathrm{SIMes})$ and $\left[\operatorname{Ir}(\mathrm{COD})\left(\mathrm{PCy}_{3}\right)(\mathrm{py})\right]^{+}$. Here, pyridine is used as an exemplary substrate compound.

Scheme 5: Substrate and Hydride exchange pathways in the active SABRE polarization transfer catalyst. Here, pyridine is employed as an exemplary substrate. $\mathrm{L}=\mathrm{PC} \mathrm{y}_{3}$, IMes, SIMes.............................. 32

Scheme 6: Synthesis strategy developed for compound 1

Scheme 7: The reaction steps of aniline protection, boronic acid formation and Pd-catalyzed coupling reaction to synthetize compound 5 in three steps, starting from 4-bromo-2,6-dimethylaniline.

Scheme 8: Cleavage of the protections groups and coupling reaction with 2,4,6-trimethylaniline to give formamidine 7 in two steps.

Scheme 9: The ring-closing reaction giving imidazolinium chloride $\mathbf{8}$ and the subsequent unsuccessful elimination reaction to reach target molecule 1.

Scheme 10: Intended reaction route for the generation of an NHC ligand by modification of functionalized polymer beads and the subsequent creation of a solid-phase bound SABRE precatalyst complex. The polymer beads represented by the grey spheres consist of crosslinked polystyrene.

Scheme 11: Polymer-bound ligands 18 and 19 and their corresponding iridium complexes 20 and 21 


\section{List of Abbreviations}

Ac

ALTADENA

alignment

bipy

$\mathrm{Bu}$

$\mathrm{Bz}$

cat

COD

COSY

$\mathrm{CW}$

$\mathrm{Cy}$

DC

DIPEA

DFT

DNA

DNP

DMF

DMSO

eq

Et

EXAFS

EXSY

IMes

INEPT

IPr

IPSO

LAC

LC

LIGHT-SABRE

Me

Mes

MEOP

MRI
Acetyl

Adiabatic longitudinal transfer after dissociation engenders nuclear

2,2'-Bipyridine

Butyl

Benzyl

catalytic

Cyclooctadiene

Correlation spectroscopy

Continuous wave

Cyclohexyl

Direct current

Diisopropylethylamine

Density functional theory

Deoxyribonucleic acid

Dynamic nuclear polarization

$\mathrm{N}, \mathrm{N}$-dimethylformamide

Dimethyl sulfoxide

equivalent

Ethyl

Extended $\mathrm{x}$-ray absorption fine structure

Exchange spectroscopy

1,3-Bis(2,4,6-trimethylphenyl)imidazol-2-ylidene

Insensitive nuclei enhanced by polarization transfer

1,3-Bis(2,6-diisopropylphenyl)imidazol-2-ylidene

Intelligent pulse sequence organizer

Level anti-crossing

Level crossing

Low irradiation generation of high tesla signal amplification by reversible exchange

Methyl

Mesityl

Metastability exchange optical pumping

Magnetic resonance imaging 
List of Abbreviations

\begin{tabular}{|c|c|}
\hline $\mathrm{NHC}$ & $\mathrm{N}$-heterocyclic carbene \\
\hline nico & Nicotinamide \\
\hline NMR & Nuclear magnetic resonance \\
\hline OPSY & Only para-hydrogen spectroscopy \\
\hline PAAP & $\pi$-accepting ability parameter \\
\hline $\begin{array}{l}\text { PASADENA } \\
\text { alignment }\end{array}$ & Parahydrogen and synthesis allow dramatically enhanced nuclear \\
\hline $\mathrm{Ph}$ & Phenyl \\
\hline PHIP & Parahydrogen-induced polarization \\
\hline PMAA & Poly(methacrylic acid) \\
\hline $\operatorname{Pr}$ & Propyl \\
\hline PVP & Polyvinylpyridine \\
\hline Py & Pyridine \\
\hline RF & Radio frequency \\
\hline RNA & Ribonucleic acid \\
\hline SABRE & Signal amplification by reversible exchange \\
\hline SABRE-SHEATH & $\begin{array}{l}\text { Signal amplification by reversible Exchange in shield enables alignment } \\
\text { transfer to heteronuclei }\end{array}$ \\
\hline SEOP & Spin-exchange optical pumping \\
\hline SIMes & 1,3-Bis(2,4,6-trimethylphenyl)-4,5-dihydroimidazol-2-ylidene \\
\hline SNR & Signal-to-noise ratio \\
\hline TEP & Tolman electronic parameter \\
\hline THF & Tetrahydrofuran \\
\hline xS & Excess \\
\hline
\end{tabular}




\section{Pulse Programs}

\section{1 zg.ebdu_trigin}

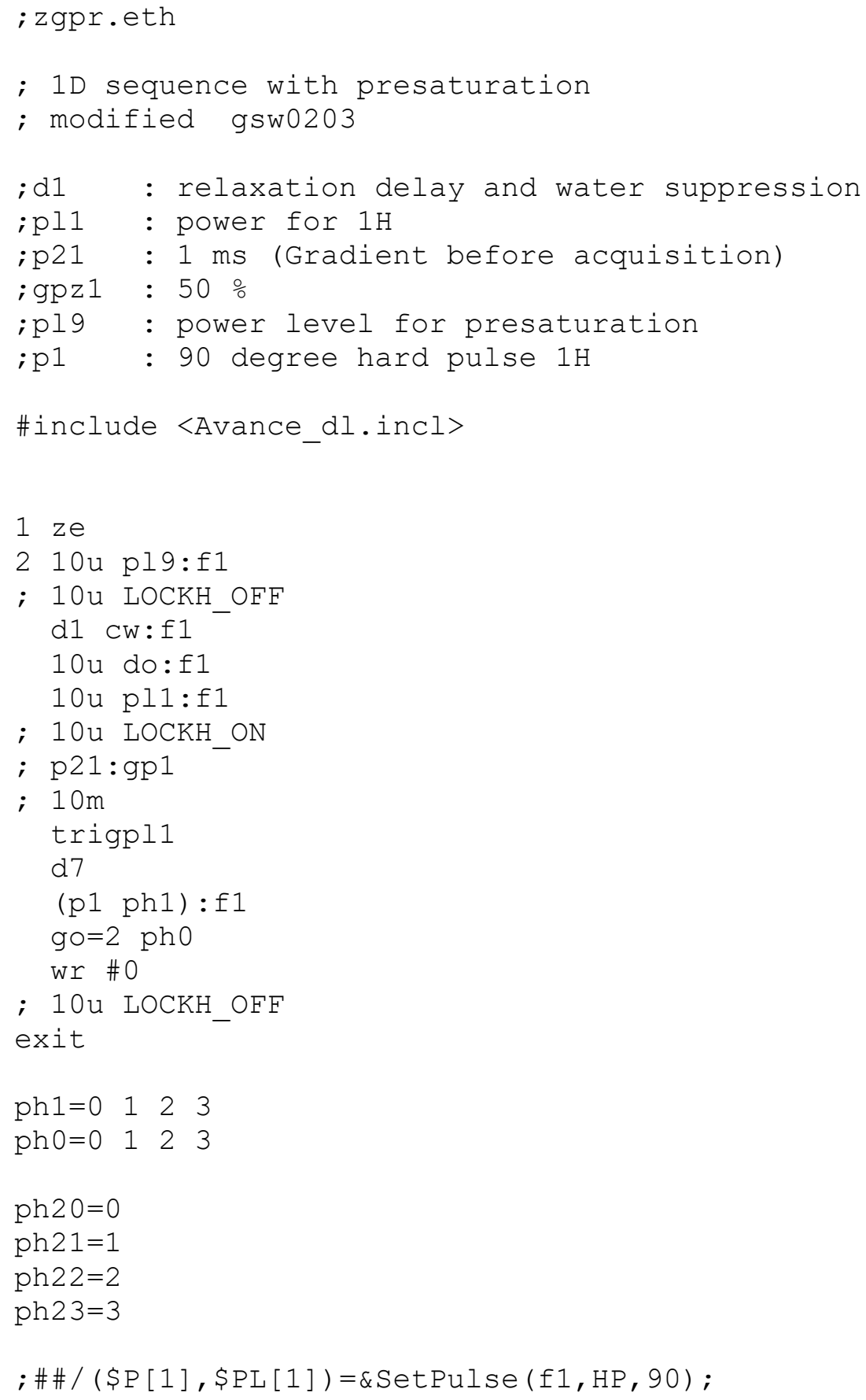




\section{Pulse Programs}

\section{2 ph_zg_noload_noempty}

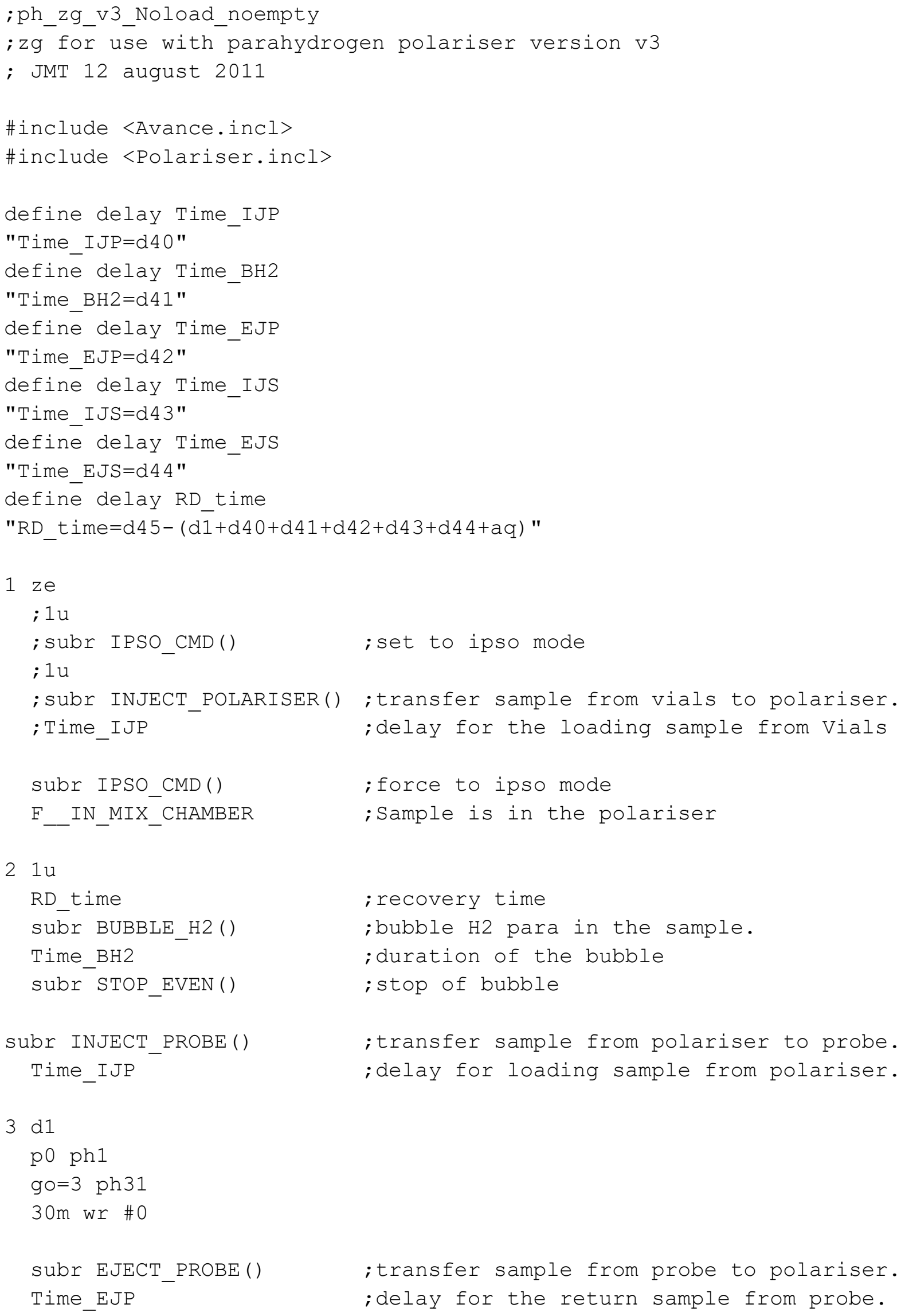


Pulse Programs

lo to 2 times 10

$; 1 \mathrm{u}$

; subr EJECT_POLARISER() ; transfer sample from polariser to vials.

; Time_EJP ; delay for eject the sample from

- subr MANUAL CMD() - iset to manual mode

exit

ph1=0 $\begin{array}{lllllllllll}2 & 2 & 0 & 1 & 3 & 3 & 1\end{array}$

ph31=0 $2 \begin{array}{lllllllll} & 2 & 0 & 1 & 3 & 3 & 1\end{array}$

;d1 : relaxation delay; set to very short for hyperpolarisation experiments

; ns : number of scans: set to $=1$ for hyperpolarisation experiments

; d40: delay for the loading sample from Vials

;d41: duration of the bubble

;d42 : delay for loading sample from polariser.

; d43 : delay for the return sample from probe.

; d44: delay for eject the sample from polariser to vials.

;10 : accumulate LO scans; re-polarising between scans 


\section{Curriculum Vitae}

\begin{tabular}{|c|c|}
\hline Personal Details & \\
\hline Name & Dücker, Eibe Behrend \\
\hline Date of Birth & 18th of October 1984 \\
\hline Place of Birth & Twistringen, Germany \\
\hline Nationality & German \\
\hline Professional Experience & \\
\hline $04 / 2011-06 / 2017$ & $\begin{array}{l}\text { Research associate } \\
\text { Max Planck Institute for Biophysical Chemistry in Göttingen } \\
\text { Department NMR-based Structural Biology }\end{array}$ \\
\hline $2008-2015$ & $\begin{array}{l}\text { Teaching assistant } \\
\text { University of Göttingen }\end{array}$ \\
\hline Education & \\
\hline $04 / 2011-05 / 2018$ & $\begin{array}{l}\text { Doctorate } \\
\text { "Enhancement Strategies in NMR Spectroscopy" } \\
\text { in the workgroup of Prof. Dr. C. Griesinger at the Max Planck Institute } \\
\text { for Biophysical Chemistry } \\
\text { Georg August University School of Science } \\
\text { Research focus: Signal enhancement in NMR spectroscopy using } \\
\text { parahydrogen }\end{array}$ \\
\hline $11 / 2017-02 / 2018$ & $\begin{array}{l}\text { Advanced Training as Certified Project Management Associate (GPM) } \\
\text { at the BUPNET GmbH in Göttingen } \\
\text { Certification IPMA Level D }\end{array}$ \\
\hline $08 / 2005-02 / 2011$ & $\begin{array}{l}\text { University education } \\
\text { Student of Chemistry } \\
\text { University of Göttingen } \\
\text { Diploma thesis: "Non-Hydrogenative Parahydrogen-Induced Polarization } \\
\text { on Pyrazole Derivatives" }\end{array}$ \\
\hline $1997-2004$ & $\begin{array}{l}\text { School education } \\
\text { Academic high school } \\
\text { Graduation with "Abitur" }\end{array}$ \\
\hline Scientific publications & $\begin{array}{l}\text { Dücker, E. B., Kuhn, L. T., Münnemann, K., Griesinger, C. (2012) Similarity } \\
\text { of SABRE field dependence in chemically different substrates. Journal of } \\
\text { Magnetic Resonance, } 214 \text { (1): 159-165. }\end{array}$ \\
\hline
\end{tabular}

